Universidad Politécnica de CARTAGENA

DEPARTAMENTO DE TECNOLOGÍAS DE LA INFORMACIÓN Y COMUNICACIONES

Doctorado en Tecnologías de la Información y las Comunicaciones

UNIVERSITÉ DES SCIENCES ET TECHNOLOGIES DE LILLE

École Doctorale Sciences Pour L'INGÉNIEur

Doctorat en Micro et Nano Technologies, Acoustique et Télécommunications
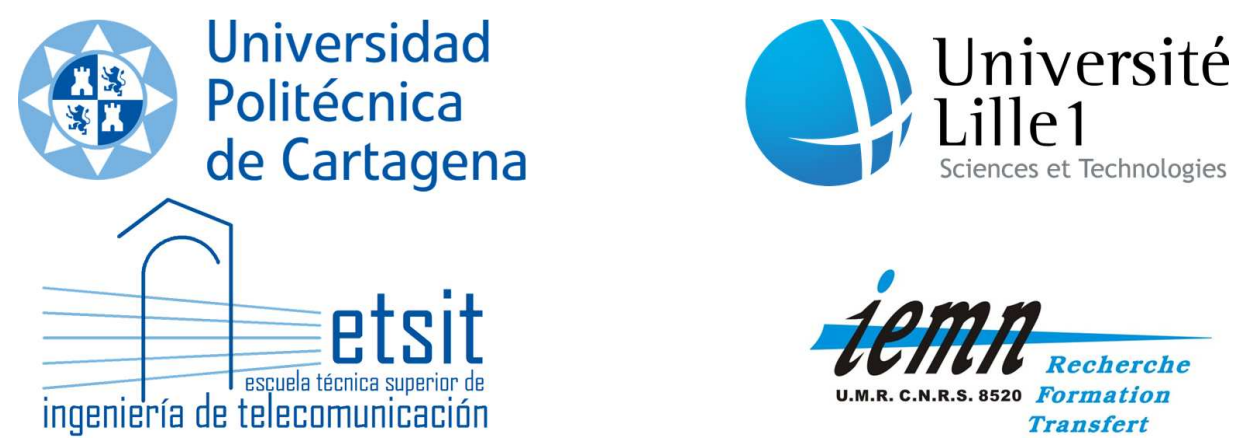

PHD DISSERTATION

\title{
Experimental Characterization of the \\ Radio Channel for Systems with Large Bandwidth and Multiple Antennas
}

Author:

Concepción García Pardo

Supervisors:

Dr. D. José María Molina García-Pardo ${ }^{(1)}$

Prof. D. Leandro Juan Llácer ${ }^{(1)}$

Prof. Mme. Martine Liénard ${ }^{(2)}$

(1) Universidad Politécnica de Cartagena (UPCT)

(2) Université des Sciences et Technologies de Lille (USTL) 


\section{Universidad \\ Politécnica \\ de Cartagena}

DT-12

\section{CONFORMIDAD DE SOLICITUD DEAUTORIZACIÓN DE DEPÓSITO DE TESIS DOCTORAL POR EL/LA DIRECTOR/A DE LA TESIS}

D. José María Molina García-Pardo Director de la Tesis doctoral Experimental Characterization of the Radio Channel for Systems with Large Bandwidth and Multiple Antennas

\section{INFORMA:}

Que la referida Tesis Doctoral, ha sido realizada por D ${ }^{a}$. Concepción García Pardo, dando mi conformidad para que sea presentada ante la Comisión de Doctorado.

La rama de conocimiento por la que esta tesis ha sido desarrollada es:

Ciencias

Ciencias Sociales y Jurídicas

叉 Ingeniería y Arquitectura

En Cartagena, a 1 de Diciembre de 2011

LOS/LAS DIRECTORES/AS DE LA TESIS

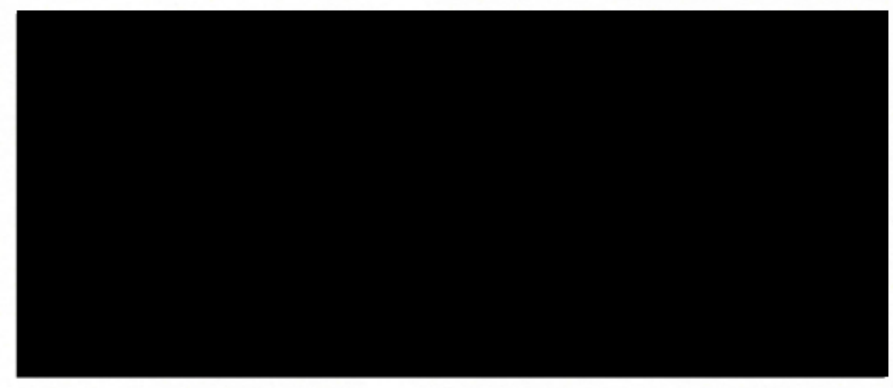




\title{
Universidad
}

Politécnica

de Cartagena

DT-12

\section{CONFORMIDAD DE SOLICITUD DEAUTORIZACIÓN DE DEPÓSITO DE TESIS DOCTORAL POR EL/LA DIRECTOR/A DE LA TESIS}

D. Leandro Juan Llácer Director de la Tesis doctoral Experimental Characterization of the Radio Channel for Systems with Large Bandwidth and Multiple Antennas

\section{INFORMA:}

Que la referida Tesis Doctoral, ha sido realizada por Da . Concepción García Pardo, dando mi conformidad para que sea presentada ante la Comisión de Doctorado.

La rama de conocimiento por la que esta tesis ha sido desarrollada es:

\author{
$\square \quad$ Ciencias \\ C Ciencias Sociales y Jurídicas \\ 邓 Ingeniería y Arquitectura
}

En Cartagena, a 1 de Diciembre de 2011

LOS/LAS DIRECTORES/AS DE LA TESIS

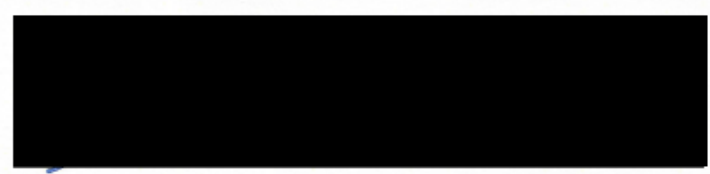

Fdo.: LEANORS NAN LCACER 


\section{Universidad}

Politécnica

de Cartagena

DT-12

\section{CONFORMIDAD DE SOLICITUD DEAUTORIZACIÓN DE DEPÓSITO DE TESIS DOCTORAL POR EL/LA DIRECTOR/A DE LA TESIS}

$D^{a}$. Martine Liénard Directora de la Tesis doctoral Experimental Characterization of the Radio Channel for Systems with Large Bandwidth and Multiple Antennas

\section{INFORMA:}

Que la referida Tesis Doctoral, ha sido realizada por Da . Concepción García Pardo, dando mi conformidad para que sea presentada ante la Comisión de Doctorado.

La rama de conocimiento por la que esta tesis ha sido desarrollada es:

$\square \quad$ Ciencias

$\square \quad$ Ciencias Sociales y Jurídicas

X Ingeniería y Arquitectura

En Cartagena, a 1 de Diciembre de 2011

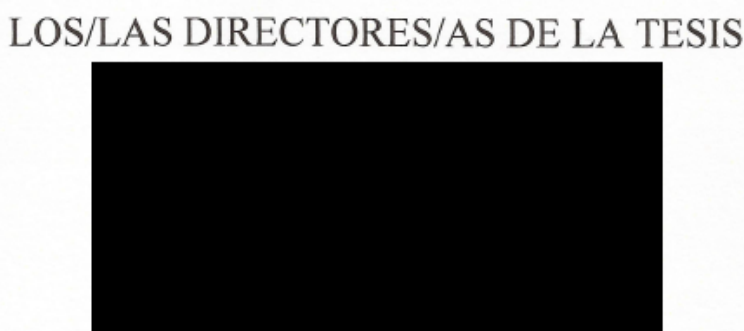

Fdo.: Martine Liénard 


\section{CONFORMIDAD DE DEPOSITO DE TESIS DOCTORAL POR LA COMISIÓN ACADÉMICA DEL PROGRAMA}

D/D ${ }^{a}$. Francisco J. Ortiz Zaragoza, Presidente/a de la Comisión Académica del Programa Tecnologías de la Información y las Comunicaciones

\section{INFORMA:}

Que la Tesis Doctoral titulada, "Experimental Characterization of the Radio Channel for Systems with Large Bandwidth and Multiple Antennas", ha sido realizada por $\mathrm{D}^{\mathrm{a}}$ Concepción García Pardo bajo la dirección y supervisión del Dr. José María Molina García-Pardo, Dr. Leandro Juan Llácer y Dra. Martine Liénard dando su conformidad a la misma la Comisión Académica, con la finalidad de que sea presentada ante la Comisión de Doctorado.

La Rama de conocimiento por la que esta tesis ha sido desarrollada es:

$\square$ Ciencias

$\square$ Ciencias Sociales y Jurídicas

$\square$ Ingeniería y Arquitectura

En Cartagena, a 5 de Diciembre de 2011

EL PRESIDENTE DE LA COMISIÓNACADÉMICA DEL PROGRAMA

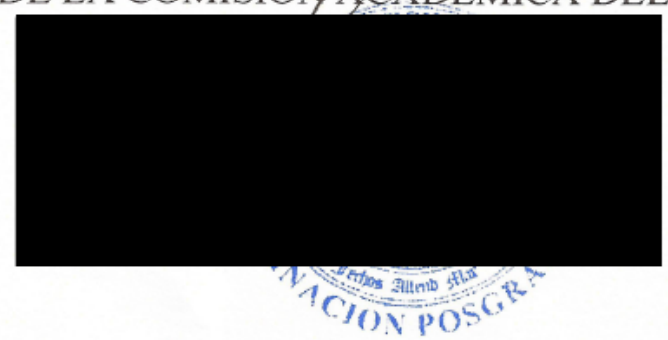

COMISIÓN DE DOCTORADO 

This thesis has been developed under the co-tutelage agreement between by the research group of Sistemas de Comunicaciones Móviles, SiCoMo, from the Universidad Politécnica de Cartagena, UPCT (Spain), and the research group of Télécommunication, Interférences et Compatibilité Electromagnétique, TELICE, from the Université des Sciences et Technologies de Lille, USTL-Lille 1 (France), signed in October of 2008. 



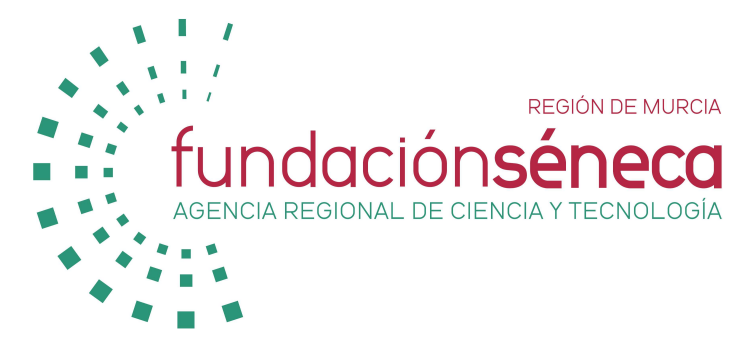

This thesis has been funded thanks to a pre-doctoral grant given by the Fundación Séneca, Agencia Regional de Ciencia y Tecnología de la Región de Murcia: 06640/FPI/07, 08451/EFPI/08, 10753/EFPI/09, 14809/EFPI/10. 



\section{ACKNOWLEDGEMENTS}

Ortega y Gasset, a Spanish philosopher, said: "I am me and my circumstances". I agree completely. This thesis is also a part of the people who have surrounded me during my life and during these four years. I would like to thank so many people... I will try to do it along the following lines.

I would like to start with the Spanish part of this thesis, the Universidad Politécnica de Cartagena, where I started to work in 2007.

Firstly, I would like to thank my Spanish supervisors for having given me the opportunity and the means for arriving here. I would like to thank José María Molina for his patience and his dedication to me during all these years. I would also like to thank him for his availability at almost any time of the day and of the year to solve my doubts or supervise my work. Thank you for all I have learnt from you. I would like to thank Leandro Juan for his help and availability since I started my final degree project in 2006. I would like to thank him for his optimism and enthusiasm for the work, his patience and availability for all I have needed.

I could never forget to thank my colleagues from the SiCoMo research group: María, Mayte, José Víctor, Juan Pascual, Ricardo and Fran. Everything seems easier when you work with such fantastic people. Especially I would like to thank María and Mayte for the confidences and laughs of the last years. Thank you for making each day different in our laboratory at the underground. 
I do not want to forget the technical staff of the department: Noelia, Marga, Eva and Marcos. I would like to thank them for making my work easier. Especially I would like to thank Noelia each UWB antenna perfectly repaired or her speed providing me with the equipment I needed. Thank you all for helping every day.

I would like to thank the colleagues from the GEAT research group for their friendship and for making me feel like another GEAT member. I would like to thank my PhD colleagues Alejandro, Jose Lorente, Monica, Maria, Raúl... Especially, I would like to thank Sebas for helping me start writing in LaTeX. Thanks as well to associate professor Fernando Quesada for helping me with the lessons during the last months so I have been able to finish this thesis quicker.

Finally, I would like to thank all the Departamento de Tecnologías de la Información y las Comunicaciones, from its head, Juan Morales, to the secretary, Ana Rodríguez. Thank you everybody for helping me in everything I have needed.

However, the work of this thesis has been divided into two countries. Therefore, I can not forget what has happened on the other side of the Pyrinees, at the Universite des Sciences et Technologies de Lille and particularly at the TELICE research group.

Firstly, I would like to thank my third supervisor and head of the TELICE group, Martine Liénard, for her availability and help throughout this thesis. I would like to thank her dedication during all the months I have spent in Lille and her help on the "non-technical" things that one appreciates very much while being abroad.

I would also like to thank Pierre Degauque for sharing his knowledge and experience with me, as well as for his dedication with the work and his patience.

I do not want to forget to thank the secretary of the TELICE group, Emmanuelle Gillmann, for her help with a lot of administrative tasks I have needed during my stays. I would also like to thank her affection and friendship all the time I have been there.

I would also like to thank Pierre Laly for his help with the computers at TELICE, for his friendship, and for the Bienvenue Chez les Ch'tis film.

Finally I would like to thank all the members of TELICE group: Virginie, Davy, Alex... and the phd students Abdu, Paul, Samuel, Khaled, Huaqiang and so on, for making me prefer workdays to weekends. 
I would like now to acknowledge the people on the personal side of my life.

Firstly, I would like to thank my parents, Pedro and Concha, for encouraging me to do the thesis when I finished my degree. I would like to thank them also their advice, help and support in the difficult moments. If they had not supported me, this would have not been possible.

I would like to thank my two brothers, Pedro José and Enrique, for giving me always their opinion, even if it was not what I wanted to hear. Thank you for being an example of effort and welldone work for me since I was a child.

I would like to thank my nieces Martina y Blanca and my nephew Guillermo, who have the surprising availability of making me happy. Thank you for your infectious smiles through Skype when I was in Lille.

I would like to thank my friends, Cristina, Araceli, Victoria, José Luis, Cristina, Virginia... my friends from La Torre, Ana, Laura, Elena, Mateo, Juanjo... for their friendship all these years and the best moments shared (and not shared because I was abroad or writing this thesis).

Finally, but not less important, I would like to thank Fran, my partner, for everything. Thank you for encouraging me to start the thesis. Thank you for your help that evening of the spring of 2009 when I was desperate because my first measurements with the oscilloscope were completely wrong. Thank you for convincing me that in the end I would manage to do it. Thank you for all the time you have spent on the telephone talking with me and listening to my inconveniences while I was in Lille. Thank you for all the hours you will spend listening to me prepare the presentation of this thesis. THANK YOU FOR EVERYTHING.

Thank you everybody. This thesis is part of all of you. 


\section{PREFACE}

Nowadays, wireless communications are a part of our everyday life. The necessity of being communicated everywhere and at any time makes users to demand faster and enhanced communications every day. Achieving this improvement in mobile communications involves the development and testing of new wireless communications systems. In particular, it has been demonstrated that, systems with large bandwidth and the use of multiple antennas provides higher data rates and more efficient communications. Furthermore, the knowledge of the radio channel is an essential issue to design new devices and, thus, reach such improvement in wireless communications.

This thesis is focused on the experimental characterization of the radio channel. This characterization is mainly focused on systems with large bandwidth and multiple antennas. Such characterization will be done by means of the analysis of measurements of the radio channel. Performing these measurements requires, first of all, the development of suitable channel sounders. Next, measurement campaigns are carried out in order to characterize the radio channel in several environments such as indoor and tunnels. Additionally, some techniques that can improve the performance of the radio link are evaluated. 


\section{CONTENTS}

1. Introduction

1.1. Motivation and Objectives

1.2. Thesis Overview

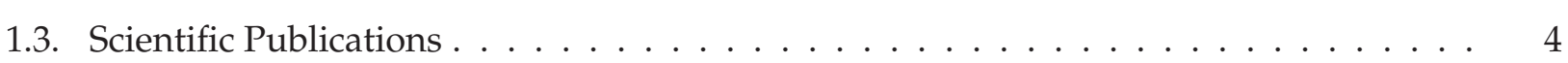

1.4. Academic Contributions ...................... ฤ

1.5. Cotutelle Framework . . . . . . . . . . . . . . . . . . . 8

2. MIMO Channel Sounding

2.1. State of the Art of Channel Sounding . . . . . . . . . . . . . . . 10

2.1.1. SISO Sounding Techniques . . . . . . . . . . . . . . . . . 10

2.1.2. MIMO Sounding Techniques ...................... 12

2.2. Frequency-Domain MIMO Channel Sounder . . . . . . . . . . . . . . . 14

2.2.1. High Spatial Resolution Channel Sounding . . . . . . . . . . . . . . . . 16 
2.2.1.1. XY Positioning Board and Step Motors . . . . . . . . . . . . . . 16

2.2.1.2. Software ............................ 17

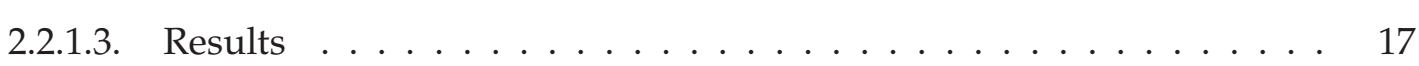

2.2.2. GIS MIMO Channel Sounding Application . . . . . . . . . . . . . . . . 24

2.2.2.1. Implementation . . . . . . . . . . . . . . . . 24

2.3. Time-Domain MIMO Channel Sounder . . . . . . . . . . . . . 26

2.3.1. Loosely-Synchronous Codes . . . . . . . . . . . . . . . . . . 28

2.3.2. Implementation . . . . . . . . . . . . . . . . . . . . . 31

2.3.2.1. Hardware Equipment . . . . . . . . . . . . . . 32

2.3.2.2. Software Application .................. 36

2.3.2.3. Synchronization and Delay Compensation . . . . . . . . . . . . 37

2.3.2.4. Dynamic Range . . . . . . . . . . . . . . . . . . 38

2.3.2.5. Post-Processing . . . . . . . . . . . . . . . . . . 39

2.3.3. Validation of the Channel Sounder . . . . . . . . . . . . . . . . 40

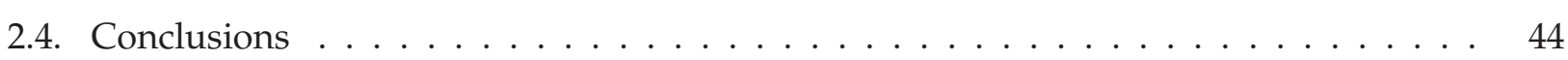

3. Polarized MIMO-UWB Channel Modeling

3.1. State of the Art of UWB Channel Modeling . . . . . . . . . . . . . . . 46

3.1.1. Polarized MIMO-UWB Channel Modeling . . . . . . . . . . . . . . . . . . . 47

3.2. Description of the Measurement Campaign . . . . . . . . . . . . . 48

3.3. Path Loss Models . . . . . . . . . . . . . . . . . . . . . . . . . . . . . . 51

3.3.1. Propagation along the Corridor . . . . . . . . . . . . . . . . 52

3.3.1.1. Analysis of Co-polar Polarizations .............. 52

3.3.1.2. Analysis of Cross-polar Polarizations . . . . . . . . . . . . . . 56

3.3.2. Through-the-wall Propagation in Labs . . . . . . . . . . . . . . . . . . 5 58 
3.3.2.1. Analysis of Co-polar Polarizations . . . . . . . . . . . . . . . . 59

3.3.2.2. Analysis of Cross-polar Polarizations . . . . . . . . . . . . . . . . 60

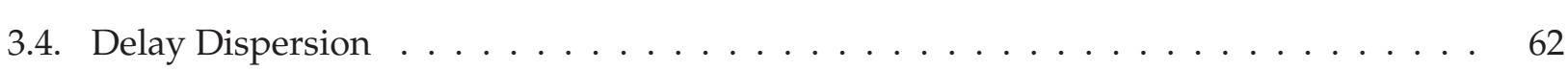

3.4.1. Delay Dispersion in LoS and NLoS along the Corridor . . . . . . . . . . . . 63

3.4.1.1. Analysis in Space Domain . . . . . . . . . . . . . . . . . 63

3.4.1.2. Analysis in Frequency Domain . . . . . . . . . . . . . . 64

3.4.2. Delay Dispersion in Labs . . . . . . . . . . . . . . . . . . . . 65

3.4.2.1. Analysis in Space Domain . . . . . . . . . . . . . . . . . 65

3.4.2.2. Analysis in Frequency Domain . . . . . . . . . . . . . . . 66

3.5. Evaluation of the MIMO System Capacity . . . . . . . . . . . . . . . 66

3.5.1. Capacity for Constant Received power . . . . . . . . . . . . . . . . . 67

3.5.1.1. Capacity along the Corridor in $\operatorname{LoS} \ldots \ldots \ldots$

3.5.1.2. Capacity along the Laboratories . . . . . . . . . . . . . . . . . 69

3.5.2. Capacity for Constant Transmitted power . . . . . . . . . . . . . 70

3.5.2.1. Capacity along the Corridor in $\operatorname{LoS} \ldots \ldots \ldots \ldots$

3.5.2.2. Capacity across the Laboratories . . . . . . . . . . . 71

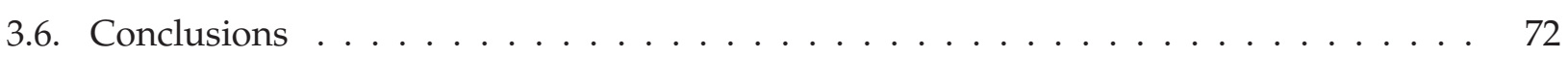

4. Multi-Dimensional Characterization in Tunnels $\quad 75$

4.1. State of the Art of Channel Modeling in Tunnels . . . . . . . . . . . . . 76

4.1.1. Theoretical Characterization $\ldots \ldots \ldots \ldots \ldots \ldots \ldots$

4.1.1.1. Ray Tracing Approach ................... 77

4.1.1.2. Modal Theory Approach ................ 78

4.1.2. Diversity Techniques . . . . . . . . . . . . . . . . . . . 80

4.1.2.1. Frequency Diversity . . . . . . . . . . . . . . . 80 
4.1.2.2. Polarization Diversity . . . . . . . . . . . . . . 80

4.1.2.3. Time-Reversal Technique . . . . . . . . . . . . . . . . . 81

4.2. Description of the Measurement Campaign . . . . . . . . . . . . 83

4.2.1. Measurement Equipment . . . . . . . . . . . . . . . . . . 83

4.2.2. Methodology ... . . . . . . . . . . . . . . . . 85

4.2.3. Polarization ...................... 86

4.3. Experimental UWB Channel Characterization . . . . . . . . . . . . . . . . 86

4.3.1. Mean Path Loss and Small Scale Fading . . . . . . . . . . . . . . . . . . . 86

4.3.2. Channel Characterization in Space-Delay-Frequency Domains . . . . . . . . . . 88

4.3.2.1. Correlation ....................... 88

4.3.2.2. Power Delay Profile and Delay Spread . . . . . . . . . . . . . . . . 90

4.3.2.3. Angular Power Spectrum and Angular Spread . . . . . . . . . . . . 94

4.3.2.4. Frequency Selectivity . . . . . . . . . . . . . . . . . 98

4.4. Diversity Techniques ... . . . . . . . . . . . . . . . . . . . 999

4.4.1. Frequency Diversity . . . . . . . . . . . . . . . . . . 100

4.4.1.1. Space-Frequency Diversity . . . . . . . . . . . . . . . . 101

4.4.2. Polarization Diversity . . . . . . . . . . . . . . . . . . 103

4.4.2.1. Capacity for Constant Received Power . . . . . . . . . . . . . . . 103

4.4.2.2. Capacity for Constant Transmitted Power . . . . . . . . . . . . . 105

4.4.3. Space-Delay Diversity: Time Reversal . . . . . . . . . . . . . . . . . . . . . 107

4.4.3.1. Transmitted and Received Waveforms . . . . . . . . . . . . . 107

4.4.3.2. Time Reversal . . . . . . . . . . . . . . . . . . . . . . 109

4.4.3.3. Spatial Focusing of SISO-TR . . . . . . . . . . . . . . 111

4.4.3.4. Temporal Focusing: Comparison between SISO-TR and MISO-TR . . 112 
4.4.3.5. Some Time-Reversal Characteristics . . . . . . . . . . . . . 113

4.5. Conclusions ............................ 115

5. Conclusions and Perspectives $\quad 119$

5.1. Conclusions ............................... 120

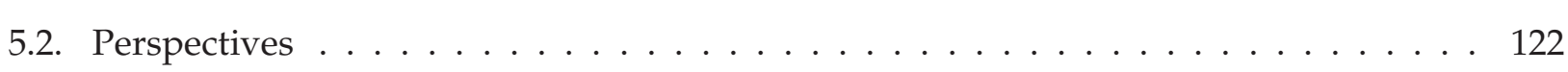

\section{Appendices}

A. Multiple-Input Multiple-Output (MIMO)

A.1. Multiantenna Systems . . . . . . . . . . . . . . . . . . 125

A.2. MIMO Benefits . . . . . . . . . . . . . . . . . . . . 127

A.3. Correlation ............................... 128

A.4. Ergodic Capacity and Outage . . . . . . . . . . . . . . . . . . . . 129

B. Characterization of the Wideband Radio Propagation Channel

B.1. Large-Scale Fading . . . . . . . . . . . . . . . . . . . . . . . 132

B.2. Small-Scale Fading . . . . . . . . . . . . . . . . . . 132

B.3. Characterization of Deterministic and Random Channels . . . . . . . . . . . . 133

B.4. Classification of Practical Channels . . . . . . . . . . . . . . . . . 134

B.5. Parameters of Mobile Multipath Channels . . . . . . . . . . . . . . . 135

B.5.1. Path Loss and Shadowing . . . . . . . . . . . . . . . . . . . . 135

B.5.2. Delay Dispersion Parameters . . . . . . . . . . . . . . . . . 136

B.5.3. Coherence Bandwidth . . . . . . . . . . . . . . . . . 137

B.5.4. Doppler Spread and Coherence Time . . . . . . . . . . . . . . . . . . . 137

B.5.5. Angular Spread . . . . . . . . . . . . . . . . . . . . . . . . 138 
\begin{tabular}{l|l|} 
C. Frequency-Domain Channel Sounder & 139 \\
\hline
\end{tabular}

C.1. System Description . . . . . . . . . . . . . . . . . . . . . . . . . . 140

C.1.1. Hardware ............................ 140

C.1.1.1. Multiport Vector Network Analyzer . . . . . . . . . . . . . . . 140

C.1.1.2. Switch and Controller . . . . . . . . . . . . . . . 141

C.1.1.3. Fiber Optic Link . . . . . . . . . . . . . . . . . . . . 141

C.1.1.4. Computer......................... 142

C.1.1.5. Others ............................... 142

C.1.2. Software ................................. 142

C.2. Measurement Process . . . . . . . . . . . . . . . . . . . . . . . 143

C.2.1. Calibration ........................... 144

C.2.2. Measurement Routine . . . . . . . . . . . . . . . . . . . . 144

D. Geographic Information Systems

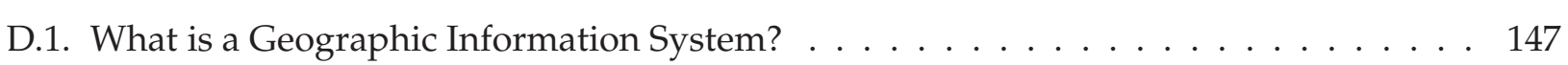

D.2. Data Representation . . . . . . . . . . . . . . . . . . . . 148

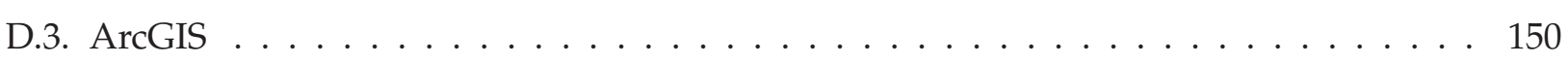

D.3.1. ArgGIS Desktop, ArcView and ArcMap . . . . . . . . . . . . . . . . 151

\begin{tabular}{l|l|}
\hline List of Figures & 153 \\
\hline
\end{tabular}

\begin{tabular}{l|l|} 
List of Tables & 159 \\
\hline
\end{tabular}

\begin{tabular}{l|l|} 
Bibliography & 161 \\
\hline
\end{tabular}

\begin{tabular}{l|l} 
Glossary & 173 \\
\hline
\end{tabular} 
Chapter 1

\section{INTRODUCTION}

\subsection{Motivation and Objectives}

Wireless communications have become essential in our society [Rappaport, 1996], [Parsons, 2000]. Nowadays, people need to be connected everywhere and at any time, and demand faster and enhanced communications every day. New applications requires higher data rates and, therefore, higher bandwidths.

On the one hand, Multiple-Input Multiple-Output (MIMO) systems were proposed as one solution to achieve higher data rates and optimize the use of the spectrum. On the other hand, more recently, systems with an ultra large bandwidth, and particularly Ultra-Wideband (UWB) systems, have gained the interest of the scientific community. Such interest is owing to the extremely high data rates offered and its possible coexistence with existing systems due to the its low transmitted power.

However, this improvement in mobile communications involves the development and testing of new wireless communications systems. Precise knowledge of the radio channel is an essential issue to design this new devices and, thus, reach such improvement in wireless communications.

In general, the modeling of the radio channel can be undertaken in two main ways: 
- Theoretically, where the channel is characterized by means of simulations and theoretical approaches.

- Experimentally, where the radio channel is characterized by means of the analysis of measurements carried out in real scenarios.

This thesis is mainly focused on the experimental characterization of the radio channel for systems with large bandwidth and multiple antennas (MIMO). However, characterizing experimentally the MIMO wideband channel implies the availability of adequate and accurate channel sounders.

There are many parameters that characterizes the radio channel such as the path loss, the RMS delay spread, the coherence bandwidth, and so on. If different polarizations in the receiving and transmitting arrays are used, the Cross-Polar Discrimination factor (XPD) plays also an important role in the channel characterization. The complex correlation coefficient or the capacity define also the performances of multiantenna systems. All these parameters can be deduced from experimental measurements. Thus, the particular behavior of the radio channel where measurements were carried out can be observed.

In general, most of research for MIMO and UWB are performed in indoor environments. However, there are scenarios where reliable communications are needed, for example, in tunnels. In this special environment there is an increasing necessity of developing and implementing control/command equipment, signalling systems and new information and communication technologies in order to develop safe and efficient trains and infrastructures. Consequently, a good characterization of the radio channel is again required for this environment.

Thus, the objectives pursued in this thesis are:

1. The improvement of an existing frequency-domain MIMO channel sounder to achieve more enhanced capabilities.

2. The development of a wideband time-domain MIMO channel sounder in order to perform measurements directly in the time-domain.

3. Characterize the MIMO-UWB radio channel in office environment by means of measurements carried out in the underground level of the Universidad Politécnica de Cartagena. To accomplish this, the influence of space and frequency on single antenna parameters is evaluated. Mutiantenna parameters such as the MIMO capacity are also deduced from these measurements.

4. Study the propagation in tunnels by analyzing some classical single antenna parameters. 
5. Evaluate the performance of applying frequency and polarization diversity techniques as well as the Time-Reversal technique in tunnels.

In the following section the organization of this work to comply with the objectives above presented is explained.

\subsection{Thesis Overview}

In this section the organization and presentation of the work of this thesis is summarized. However, due to the wide variety of topics treated, each chapter includes a brief review of the state of the art focused on the specific research field treated on it.

Chapter 2 is devoted to the improvement and development of MIMO channel sounders. Firstly, a brief state of the art about Single-Input Single-Output (SISO) and MIMO sounding techniques is presented. Next, some modifications performed on an existing MIMO frequency-domain channel sounder are proposed. The first one, includes a positioning system which can measure the channel with a high spatial resolution. In this case, some results from a small measurement campaign are provided. The second one is the development of a software to georeference all the measurement campaigns. To accomplish this, a Geographic Information System (GIS) is used. Finally, in this chapter a new MIMO time-domain channel sounder is proposed. This measurement system is based on Code-Division Multiplexing (CDM) and it uses commercial multipurpose equipment, which is one of its main advantages. Measurements in the same scenario are carried out with this new system and with the one existing in the frequency domain. Results are compared in order to validate the correct operation of the time-domain channel sounder.

In Chapter 3, the polarized MIMO-UWB radio channel is studied experimentally by analyzing measurements from a indoor measurement campaign in an office environment. Firstly, the state of the art about UWB channel modeling is reviewed. Secondly, the channel sounder, the measurement environment and the measurement configuration are detailed. Next, the path loss is analyzed in both space and frequency domains. Since polarization is taken into account in measurements, the effect of distance and frequency on the Cross-Polar Discrimination factor (XPD) is also considered. Next, the RMS delay spread is also evaluated in order to characterize the delay dispersion of the UWB channel in office environments. Again, this analysis is carried in both space and frequency domains. Finally, the MIMO system capacity is evaluated under the assumption of constant transmitted or received power (variable or constant SNR respectively).

The last chapter of this thesis, the Chapter 4 , deals with the experimental characterization of the radio channel in tunnels. In this case, measurements undertaken in a UWB frequency band in an empty tunnel are used. Firstly, a review about the necessity of communications in tunnels, 
the theoretical channel characterization and some diversity techniques that can be used in this special environment are addressed. Next, the measurement equipment and the methodology are described. The experimental analysis starts with the extraction of some classical parameters such as the path loss, the complex correlation coefficient, the RMS delay spread, the angular spread or the coherence bandwidth. Finally, the possibilities of implementing some diversity techniques in tunnels are evaluated.

This thesis concludes with the Chapter 5 where the general conclusions of this thesis are summarized. The future perspectives and research lines derived from this work are also proposed in this chapter.

Furthermore, four appendices are annexed at the end of the thesis in order to treat some transversal topics and contribute to the overall comprehension of this thesis:

Appendix $\mathbf{A}$ summarizes some basic concepts about MIMO systems. In Appendix B the characterization of the radio channel and some classical single antenna parameters that describes its behavior are presented. The MIMO frequency-domain channel sounder existing in the research group is explained in depth in Appendix C. Finally, some concepts about Geographical Information Systems are addressed in Appendix D.

\subsection{Scientific Publications}

This thesis has given rise to 5 international journals (IJ) indexed in to the Journal Citation Reports, 8 international conferences (IC), 4 national journals (NJ), and 4 national conferences (NC):

Particularly, the work of Chapter 2has been published in 1 international journal, 3 international conferences, 1 national journals, and 2 national conferences:

IJ1 Concepcion Garcia-Pardo, Jose-Maria Molina-Garcia-Pardo, and Leandro Juan-Llacer. "TimeDomain MIMO Channel Sounder Based on Code-Division Multiplexing". IEEE Transactions on Instrumentation and Measurement. Status: Accepted for publication.

IC1 Concepcion Garcia-Pardo, José María Molina Garcia-Pardo, Jose Victor Rodriguez and Leandro Juan-Llacer. "A Wideband $2 \times 2$ MIMO Channel Sounder in Time-Domain and Performance Comparison with Frequency Domain Channel Sounder". COST 2100, TD(10)10035. Athens(Greece). March 2010.

IC2 C. Garcia-Pardo, J.M. Molina-Garcia-Pardo, J.-V. Rodríguez, L. Juan-Llacer. "2 $\times 2$ Real-Time Code-Division Multiplexed MIMO Channel Sounder". Proceedings of the 4th European Conference on Antennas and Propagation, EuCAP2010. Barcelona (Spain), 12- 16 April 2010. 
IC3 Concepcion Garcia-Pardo, Jose-Maria Molina-Garcia-Pardo, Jose-Victor Rodríguez, Leandro Juan-Llacer. "Comparison Between Time and Frequency Domain MIMO Channel Sounders". IEEE 72nd Vehicular Technology Conference: VTC2010-Fall. 6-9 September 2010, Ottawa, Canada.

NJ1 Concepción García Pardo, José María Molina García-Pardo, José-Víctor Rodríguez, Leandro Juan Llácer. "Sistema de Medidas de Banda Ancha en el Dominio del Tiempo y Comparación con Sistema en el Dominio de la Frecuencia". II Jornadas Introducción a la investigación (UPCT), 2009.

NC1 Concepción García-Pardo, Concepción Sanchís Borrás, J.M. Molina-García-Pardo, y L. JuanLlácer. "Sistema de Medidas en Banda Ancha en el Dominio del Tiempo y Comparación con Sistema en el Dominio de la Frecuencia". XXXIV Simposium Nacional de la URSI 2009. Santander, September de 2009.

NC2 Concepción García-Pardo, J.M. Molina-García-Pardo, J.V. Rodríguez y L. Juan-Llácer. Estudio Comparativo de "Sistemas de Medida del para Sistemas MIMO $2 \times 2$ de Banda Ancha". XXXV Simposium Nacional de la URSI 2010. Bilbao, September de 2010.

The work of Chapter 3 has been published in 1 international journals, 3 national journals, 2 international conferences and 2 national conferences:

IJ2 Concepcion Garcia-Pardo, Jose-Maria Molina-Garcia-Pardo, Andres Garrido-Cervantes, John Danson Muhehe, and Leandro Juan Llácer. Frequency Dependence of 2-5GHz Polarized UWB Channel Parameters in Office Environment. IEEE Transactions on Antennas and Propagation. Status: Accepted for publication.

IC4 J.M. Molina-García-Pardo, Concepción García-Pardo, J. V. Rodríguez y L. Juan-Llácer. "Path Loss and Delay Spread in UWB Channels". Proceedings of the 2009 IEEE International Symposium on Antennas and Propagation. Charleston (EEUU), June 2009.

IC5 C. Garcia-Pardo, M. Martinez-Quinto, M. Martinez-Ingles, J.M. Molina-Garcia-Pardo, J. V. Rodriguez, L. Juan-Llacer. "Polarimetric Analysis of the MIMO-UWB Channel in Laboratories". Convened Paper. Proceedings of the 5th European Conference on Antennas and Propagation (EUCAP2011), pp.3601-3605, 11-15 April 2011.

NJ2 Concepción García Pardo, Andrés Garrido Cervantes, José María Molina García-Pardo, JoséVíctor Rodríguez, Juan Pascual García, Leandro Juan Llácer. "Estudio de las Pérdidas de Propagación en Función de la Frecuencia en Interiores para Canales MIMO-UWB". III Jornadas Introducción a la investigación (UPCT), 2010.

NJ3 Concepción García Pardo, Ma Victoria Moreno Cano, José Ma Molina García-Pardo, José Víctor Rodríguez Rodríguez, Juan Pascual García y Leandro Juan Llácer. "Sistemas de Comunicaciones UWB". Espacio Teleco 2010. 
NJ4 Concepción García Pardo, Maria Martinez Quinto, Maria Teresa Martínez Inglés, José María Molina García-Pardo, José-Víctor Rodríguez, Juan Pascual García, Leandro Juan Llácer. "Análisis del Canal MIMO-UWB en Interiores para Diferentes Polarizaciones". IV Jornadas Introducción a la investigación (UPCT), 2011.

NC3 Concepción García Pardo, Concepción Sanchís Borras , José-María Molina García-Pardo, JoséVíctor Rodríguez, Leandro Juan Llácer. "Análisis del Canal Radio para Aplicaciones UWB". XXXIII Simposium Nacional de la URSI 2008. Madrid, September 2008.

NC4 Concepción García Pardo, José-María Molina García-Pardo, José-Víctor Rodríguez, Leandro Juan Llácer. "Análisis del Canal MIMO-UWB en Interiores para Diferentes Polarizaciones". XXXV Simposium Nacional de la URSI 2011. Madrid, September 2011.

The work of Chapter 4 has given rise to 3 international journals and 3 international conferences:

IJ3 J.M. Molina-Garcia-Pardo, M. Lienard, P. Degauque, C. Garcia-Pardo, L. Juan-Llacer. "MIMO Channel Capacity With Polarization Diversity in Arched Tunnels". IEEE Antennas and Wireless Propagation Letters, vol.8, no., pp.1186-1189, 2009.

IJ4 Concepcion Garcia-Pardo, Jose-Maria Molina-Garcia-Pardo, Martine Lienard, Davy P. Gaillot and Pierre Degauque. "Double Directional Channel Measurements in an Arched Tunnel and Interpretation Using Ray Tracing in a Rectangular Tunnel". Progress in Electromagnetic Research, PIER. Section M, vol. 22, pp. 91-107, 2012.

IJ5 Concepcion Garcia-Pardo, Martine Lienard, Pierre Degauque, Jose-Maria. Molina-GarciaPardo, Leandro Juan-Llacer. "Experimental Investigation on Channel Characteristics in Tunnel Environment for Time Reversal Ultra Wide Band Techniques". Radio Science. Status: Accepted for publication.

IC6 Concepcion Garcia-Pardo, Martine Lienard, Pierre Degauque, Jose-Maria. Molina-GarciaPardo, Leandro Juan-Llacer. "Time Domain Characterization of UWB and Time-Reversal UWB Channel in Tunnels". COST 2100, TD(10)12017. Bologna(Italy). November 2010.

IC7 Concepcion Garcia-Pardo, Jose Maria Molina Garci Pardo, Martine Lienard, Pierre Degauque. "Time Domain Analysis of Propagation Channels in Tunnels". Proceedings of the The Seventh Advanced International Conference on Telecommunications, AICT 2011. St. Maarten, The Netherlands Antilles, March 2011.

IC8 Kyoko Vin, Concepcion Garcia-Pardo, and Martine Lienard. "On the Energy Gain of Time Reversal UWB Systems". Accepted for the The 31st PIERS Conference. Kuala-Lumpur, March 2012. 


\subsection{Academic Contributions}

This thesis has given rise to 5 Final Degree Projects (PFC), and 1 Final Master Project (TFM).

Particularly, the following works are related with Chapter 2

PFC1 Development of an application to Control Remotely a Wideband MIMO Time-domain Measurement System (Desarrollo de una Aplicación para el Control Remoto de un Equipo de Medidas en el Tiempo para Sistemas MIMO de Banda Ancha). Author: Eduardo Alonso Sánchez. Supervisors: Concepción García Pardo and José María Molina Garcia-Pardo. Grade A (10). 25/03/2010.

PFC2 Implementation of an Automatic Positioning System for Automatizing the Measurements of the Radio Channel (Implementación de un Sistema se Posicionamiento se Antenas para la Automatización de Medidas del Canal Radio). Author: Verónica Alcaraz Vidal. Supervisors: Concepción García Pardo and José María Molina Garcia-Pardo. Grade A (10). 09/07/2010.

PFC3 Development of an Application Based on a Geographical Information System (GIS) for Sounding the Indoor MIMO-UWB Radio Channel (Desarrollo de una Aplicación para la Realización de Medidas del Canal MIMO-UWB en Interiores Basada en Sistemas de Información Geográfica (SIG)). Author: Carlos San Emeterio Villalaín. Supervisors: Concepción García Pardo and Leandro Juan Llácer. Grade A (10). 15/09/2011.

PFC4 Improvement of the Synchronization of a Time-Domain Wideband MIMO Measurement System (Mejora de la Sincronización de un Sistemas de Medidas MIMO de Banda Ancha en el Dominio del Tiempo). Author: Javier Solano García. Supervisors: Concepción García Pardo and José María Molina Garcia-Pardo. Grade A (10). 19/10/2011.

The following works were derived from Chapter 3:

PFC5 Experimental Analysis of the MIMO-UWB Channel in the Antiguones Ground Level (Análisis Experimental del Canal MIMO-UWB en los Sótanos se Antiguones). Author: Andrés Garrido Cervantes. Supervisors: Concepción García Pardo and José María Molina GarciaPardo. Grade A (10). 25/03/2010.

TFM1 Analysis of the Polarization Effect in Indoor Environments for MIMO-UWB Systems (Análisis del Efecto de la Polarización en Sistemas MIMO-UWB en Interiores). Author:Cesáreo Vera Garre. Supervisors: Concepción García Pardo and José María Molina Garcia-Pardo. Grade A (10). 19/10/2011. 


\subsection{Cotutelle Framework}

The work here presented is within the framework of the following research projects: TEC200766698-C04-04/TCM), from the Ministerio de Industria, Turismo y Comercio of Spain, 08818/PI/08 from the Fundación Séneca of Murcia, Spain (08818/PI/08) and TEC2010-20841-C04-03 from the Ministerio de Educación y Ciencia.

This thesis has been funded thanks to a predoctoral grant of the Fundación Séneca, Agencia Regional de Ciencia y Tecnología de la Región de Murcia, Spain (ref. 06640/FPI/07). The stages at TELICE research group in 2008, 2009 and 2010 have been funded also by the Fundación Séneca (refs. 08451/EFPI/08, 10753/EFPI/09, 14809/EFPI/10 respectively) and the Université des Sciences et Technologies de Lille in 2009. 


\section{Chapter 2}

\section{MIMO CHANNEL SOUNDING}

This chapter is devoted to the improvement and development of two channel sounders. The first one, is a Multiple-Input Multiple Output (MIMO) frequency-domain channel sounder based on a vector network analyzer and a semi-switched scheme by Time-Division Multiplexing (TDM). The second one is a MIMO time-domain channel sounder based on a high-performance oscilloscope and a fully switched scheme by Code Division Mutiplexing (CDM).

Firstly, in Section 2.1, Single-Input Single-Output (SISO) sounding techniques and MIMO sounding strategies are reviewed.

Secondly, Section 2.2 describes two main improvements performed on an existing MIMO frequency-domain channel sounder. The first one, described in Section 2.2.1, is based on the incorporation of a positioning system in order to perform high-resolution measurements in the spatial domain. The second one (Section 2.2.2) is based on the incorporation of geographic information to the measurements thanks to the use of a Geographic Information System (GIS) (see Appendix D). This feature will offer the user the possibility of manage all the measurement campaigns from a geographic point of view.

Next, the equipment that compose the MIMO time-domain channel sounder is detailed in Section 2.3.2.1. The software that controls such measurement system is described in Section 2.3.2.2 
while the synchronization and dynamic range are explained in Section 2.3.2.3 and Section 2.3.2.4 respectively. The pos-processing procedure necessary to extract the channel impulse response is described in Section 2.3.2.5. Finally, the validation of the channel sounder is presented in Section 2.3 .3 by comparing measurements provided by the existing frequency-domain channel sounder and the new time-domain channel sounder.

The main conclusions deduced from the work presented in this chapter are outlined in Section 2.4

\subsection{State of the Art of Channel Sounding}

In this section, the main SISO sounding techniques are addressed. Next, MIMO sounding techniques are classified depending on the excitation signals used or the multiantenna multiplexing strategies.

\subsubsection{SISO Sounding Techniques}

As mentioned before, to develop accurate devices for new mobile communications systems implies a precise knowledge of the radio channel. This knowledge can be achieved by means of measurements of the channel since it allows getting the necessary data to implement models of the channel as well as verify such models. The different measurement techniques of the SISO channel that can be found in literature are mainly based on the next ones [Rappaport, 1996]:

\section{A. Direct RF Pulse system}

A repetitive pulse of width $T_{b}$ is transmitted. In reception, the receiver uses a wide bandpass filter $\left(B W=2 / T_{b}\right)$, an amplifier and an envelope detector. The received signal is then displayed in an oscilloscope, as it is presented in Figure 2.1. This gives and immediate measurement of the square of the channel impulse response convolved with the probing pulse. The main advantage of this system is its simplicity. However, its main problem with this system is that it is subject to interference and noise, due to the wide passband filter. Also, the pulse system relies on the ability to trigger the oscilloscope on the first arriving signal, which can lead to a problem of synchronization.

\section{B. Spread Spectrum Sliding Correlator Channel Sounding}

A carrier signal is spread over a large bandwidth by mixing it with a binary pseudo-noise (PN) sequence having a chip duration $T_{c}$. The spread spectrum signal is then received, filtered, and despread using a PN sequence generator identical to that used at the transmitter. Although the two $\mathrm{PN}$ sequences are identical, the transmitter chip clock is run at a slightly faster rate than the receiver chip clock. When the PN code of the faster chip clock catches up with the PN code of the slower chip 


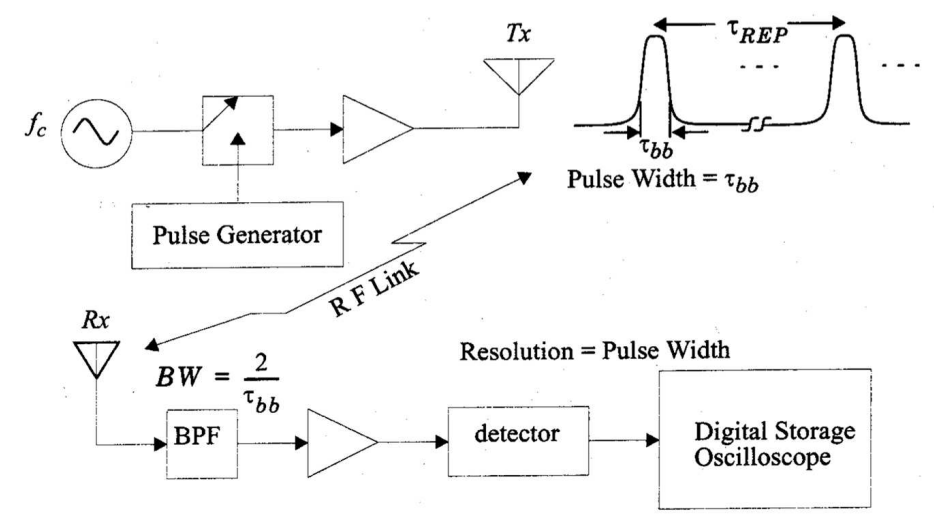

Figure 2.1. Direct RF Pulse Scheme [Rappaport, 1996].

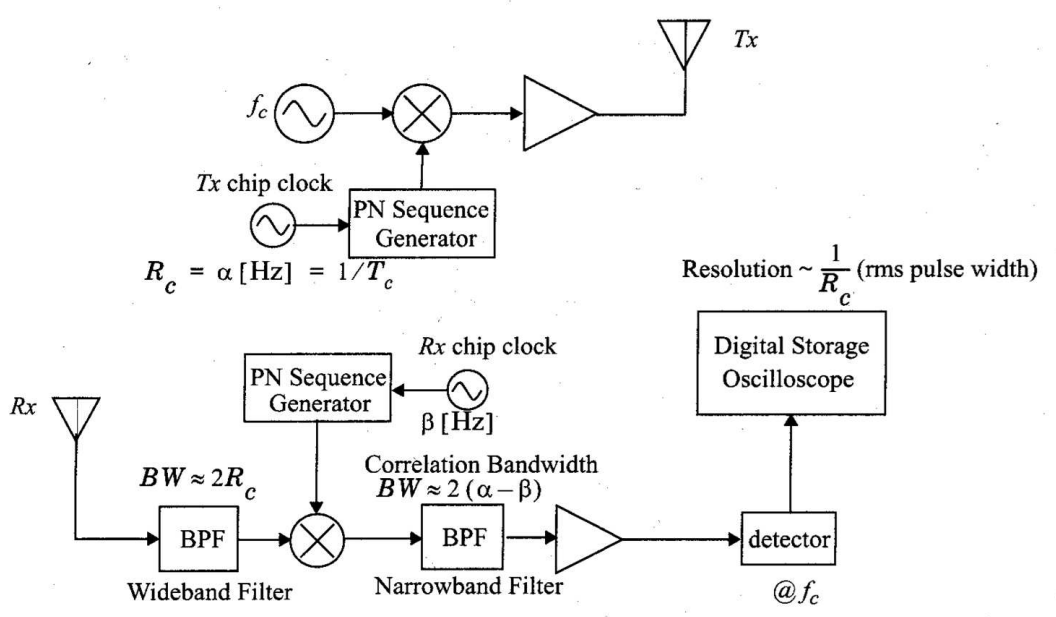

Figure 2.2. Spread Spectrum Sliding Correlator Channel Sounding [Rappaport, 1996].

clock, the two chip sequences will be virtually identically aligned, giving maximal correlation. Then, the signal is envelope detected and the channel impulse response convolved with the pulse shape of a single chip is displayed on the oscilloscope. This sounding scheme is depicted in Figure 2.2.

The main advantage of this sounding technique is the ability to reject passband noise and that the synchronization between transmitter and receiver is eliminated due to the correlation. Moreover, the required transmitter powers can be considerably lower that direct pulse systems due to the inherent processing gain of spread spectrum systems. However, the main disadvantage is that measurements are not made in real time because of the post processing procedure needed for each received signal.

\section{Frequency Domain Channel Sounding}

A vector network analyzer controls a synthesized frequency sweeper, and an S-parameter test set is used to monitor the frequency response of the channel (Figure 2.3). The sweeper scans a particular frequency band (centered on the carrier) by stepping through discrete frequencies. The number and spacing of these frequency steps impact the time resolution of the impulse response measurement. 
For each frequency step, the S-parameter test set transmits a know signal level at port 1 and monitor the received signal level at port 2 . These signal levels allow the analyzer to determine the complex response ( $S_{21}$ parameter) of the channel over the measured frequency range. The response is then converted to the time domain using inverse discrete Fourier transform (IDFT) processing, giving a band limited version of the impulse response. However, the system requires careful calibration and hardwired synchronization between the transmitter and receiver, making it useful only for very close measurements (e. fl., indoor sounding). Another limitation with this system is the non-realtime nature of the measurement. For time varying channels, the channel frequency response can change rapidly, giving an erroneous impulse response measurement.

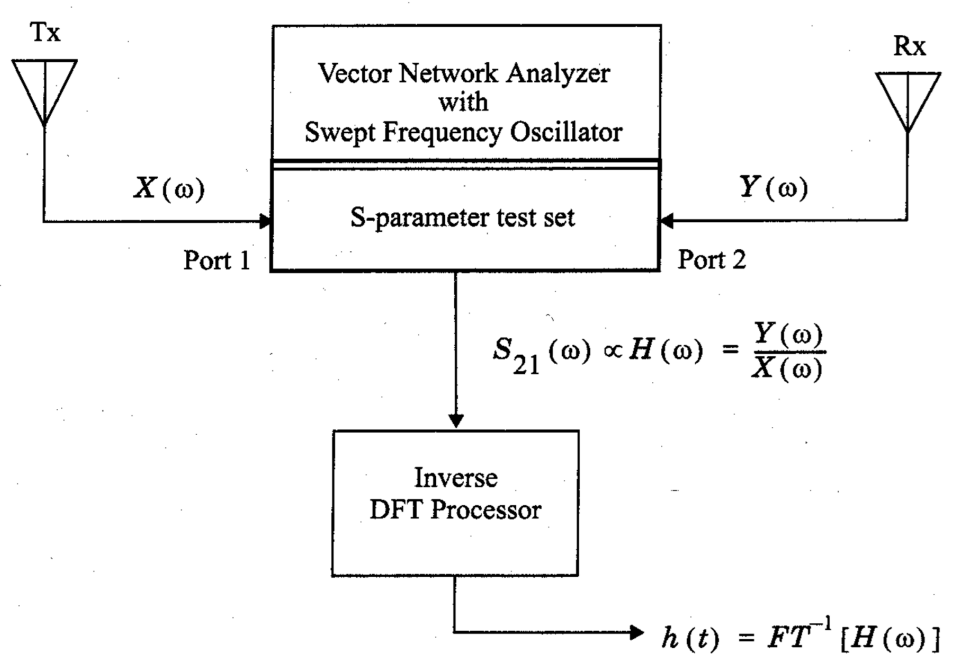

Figure 2.3. Frequency Domain Channel Sounding [Rappaport, 1996].

\subsubsection{MIMO Sounding Techniques}

MIMO sounding techniques are mainly based on the SISO sounding schemes but it should be taken into account the particularities of MIMO systems. Channel sounders should provide the temporal and spatial characteristics of the MIMO channel with accuracy and resolution high enough to benefit the design purpose. This is completely determined by channel sounding features such as the excitation signals to be considered, and the multiplexing strategies to be implemented.

\section{A. Excitation Signals}

The excitation signal choice is a key aspect in channel sounding since it determines the following signal processing to be applied and has implications on the hardware. The first sounding experiments have been carried out by using single-tone continuous wave (CW] signals. This was sufficient as long as only the narrowband channel behavior was of interest. In order to achieve high delay resolution, sequential sounding [Baum et al., 2000] at a number of different frequencies was developed. Its drawback is the resulting huge measurement time, which is not suitable for high mobility measurements. 
The pulse compression approach is well known from spread spectrum technology and makes these signals very useful for real-time identification of time-delay systems since all frequencies are instantaneously excited and a considerable Signal-to-Noise Ratio (SNR processing gain is achieved in the time domain by correlation. An example of these excitation signals are the periodic pseudo-random binary signals (PRBS) [Kivinen et al., 2001] or pseudo-random noise signals (PN) [Kivinen et al., 1999], [Peigang et al., 2004]. They can be generated easily and serve as broadband excitation signals. This is applied in the classical swept time-delay cross-correlation sounder implementation [Parsons, 2000]. The disadvantage of this principle, working sequentially, is again the long measurement time which prohibits real-time operations. Pulse compression using the chirp technique [Kemp and Bryant, 2004], [Chung et al., 2005], [Salous and Razavi-Ghods, 2004] has proven to be a powerful method for measuring the channel response since it avoids transmitter peak power limitations and offers excellent interference rejection capabilities. In general, the application of chirp signals for mobile radio channel characterization has always been limited by the capabilities of modern digital technology.

Finally, a flexible concept of excitation signals for measuring channel frequency response are multi frequency signals [Sakaguchi et al., 2002], [Thoma et al., 2000], [Maharaj et al., 2005], [Wirnitzer et al., 2001]. This approach is well known from frequency-domain system identification in measurement engineering. The advantage of this kind of signals is that channel frequency response can be measured at desired frequencies. These signals present high Peak-to-Average Power Ratio (PAPR) levels which can be reduced by numerical optimization. The difference in comparison to PRBS is that phases and magnitudes of Fourier coefficients can be arbitrarily chosen in order to optimize the system performance.

\section{B. Multiplexing Techniques}

The resolution and capability of a channel sounder is determined by the choice of the hardware architecture and multiplexing strategy. There are three multiplexing schemes for MIMO channel sounders: Time-Division Multiplexing (TDM), Code-division multiplexing (CDM) and FrequencyDivision Multiplexing (FDM).

The aim of MIMO sequential channel sounding (TDM) is to distinguish the transmit antennas as well as reducing complexity and cost. Multiple sequentially switched antenna arrays at both ends, as shown in Figure 2.4, denoted as fully-switched systems [Kivinen et al., 1999], [Wirnitzer et al., 2001], make use of a multiplexing unit that is used to step a single RF chain through all transmit/receive antenna elements sequentially in time.

In a semi-switched system [Peigang et al., 2004], [Salous and Razavi-Ghods, 2004], [Salous et al., 2005], receivers operate in parallel whereas a switching scheme is implemented at the transmitter. The use of parallel receive channels allows high resolution measurements of 


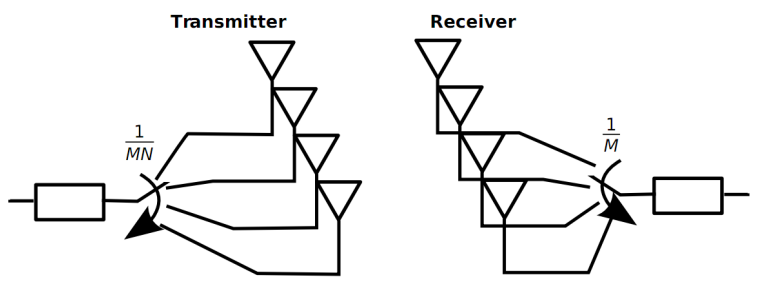

Figure 2.4. Fully-switched configuration [Laureson, 2006].

the radio environment without further reducing the effective sampling rate of the channel. This scheme is not suitable for real-time channel measurement and has some major drawbacks such as the requirement of precise synchronization between transmitter and receiver and accuracy reduction during switching time.

Switching can be avoided using parallel transmitters and parallel receivers [Takada et al., 2001] as it is presented in Figure 2.5. Clearly, the more elements used, the greater the requirement for using parallelism within the system. Moreover, the snapshot time (i.e. the time over all temporal multiplexed channels) is significantly reduced if we compare with a fully-switched scheme.
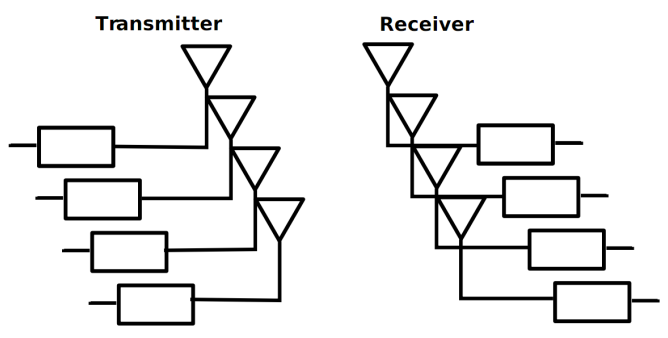

Figure 2.5. Fully-parallel configuration [Laureson, 2006].

This approach supports FDM and CDM, each with different advantages/drawbacks. With FDM, a subset of the spanned frequencies are assigned to each antenna, thus they do not all characterize the channel at exactly the same frequencies. CDM will give excellent discrimination between antenna elements provided that a low correlation between codes is maintained within the channel being sounded. Unfortunately, as the channel is a multipath one, the cross-correlation between codes at non-zero delays is important.

\subsection{Frequency-Domain MIMO Channel Sounder}

In the Research Group of Mobile Communications (SiCoMo), a frequency-domain MIMO channel sounder based on a semi-switched multiplexing scheme was developed [ibernón-Fernández et al., 2008]. As shown in Figure 2.6, such channel sounder is based on a multiport vector network analyzer (MVNA) and a fast switch. The receiving antennas are directly connected to the ports of the MVNA using coaxial cables. One port of the MVNA is configured as a 
transmitter and connected to an optical link (RF/OF and $\mathrm{OF} / \mathrm{RF}$ ), which carries the signal to the fast switch. Finally, the transmitting antennas are connected to the fast switch, so that the signal from the optical link is transmitted sequentially to each element of the transmitter array. The measurement process is controlled by a software application [Lucas Ávila, 2008] (shown in Figure 2.7) which is executed on a laptop. Such laptop is connected to the MVNA by a LAN (Local Area Network) and to the fast switch by a GPIB (General-Purpose Interface Bus) port.

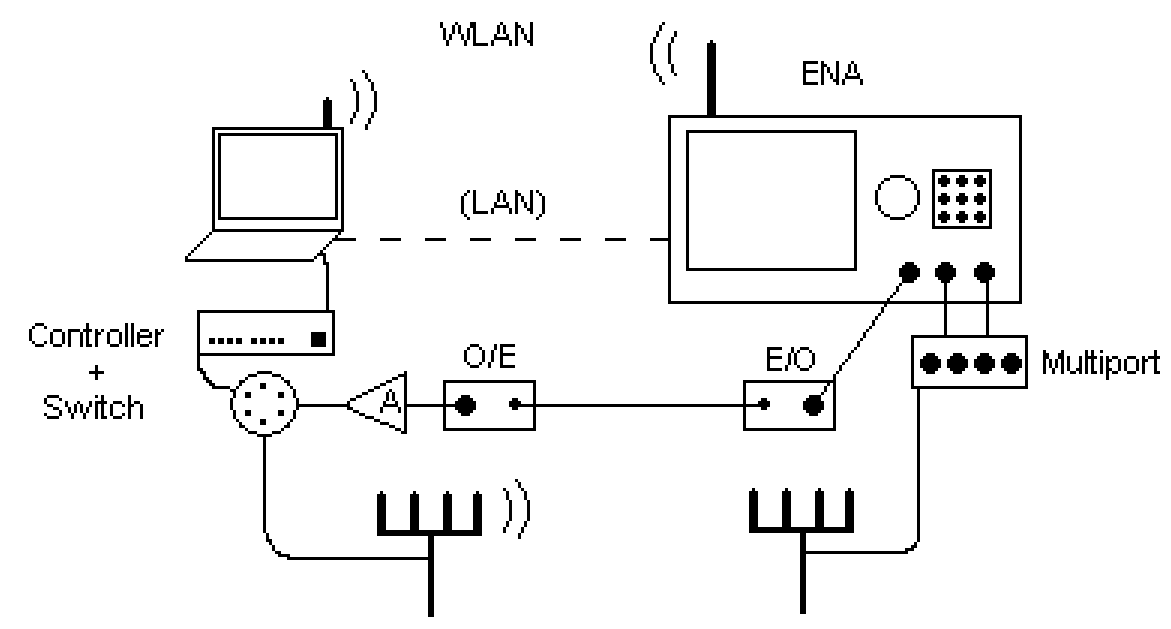

Figure 2.6. Frequency-domain channel sounder.

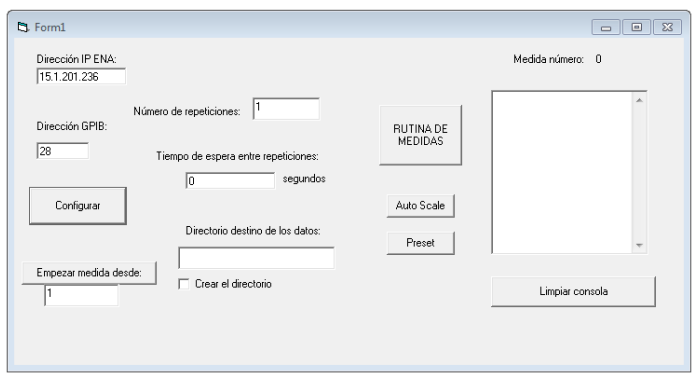

(a)

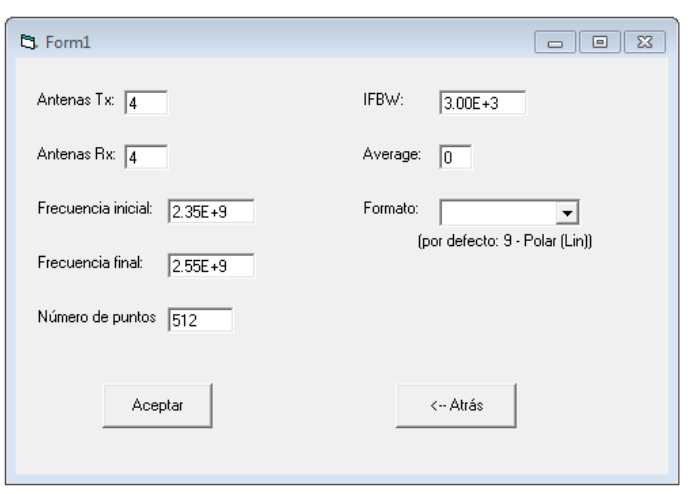

(b)

Figure 2.7. Software application [Lucas Ávila, 2008] (a) main interface, (b) configuration parameters interface.

For a more detailed information about the devices that compose this channel sounder and how it works, the reader could read Appendix C.

In the following sections, some modifications of a frequency-domain channel sounder are explained. 


\subsubsection{High Spatial Resolution Channel Sounding}

This channel sounding system is based on the use of several antennas in transmission and one single antenna for reception (MISO, Multiple-Input Single-Output). The receiving antenna is directly connected to the port number 2 of the MVNA. It is mounted in a mast, which is installed in a XY positioning board as shown in Figure 2.8. This board is composed of $X$ and $Y$ axis linear positioners and $X$ and $Y$ step motors. The hardware equipment is described in Section 2.2.1.1.

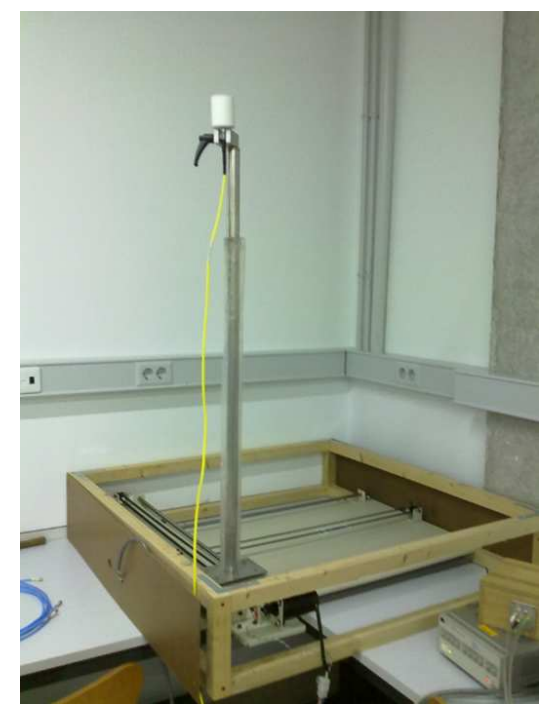

Figure 2.8. $\mathrm{XY}$ positioning board with the transmitting antenna.

The transmitting array is configured as mentioned before: the transmitting antennas are connected to the fast switch, which is also connected to the transmitting port of the MVNA via an optical link.

The application that controls the channel sounder has been modified to control the position of the mast over the board as required as described in Section 2.2.1.2.

\subsubsection{XY Positioning Board and Step Motors}

The $X Y$ positioning board consists on a board in which two linear positioners ( $X$ and $Y$ ) are installed. These positioners (see Figure 2.9) moves along $X$ and $Y$ axis by means of two $X$ and $Y$ step motors. The area covered by these positioners is $70 \times 70 \mathrm{~cm}^{2}$ in steps of at least $1 \mathrm{~cm}$. However, the main problem of a too small step is its slowness.

Once the $X Y$ positioning board has been defined, a system which moves the $X Y$ linear positioners is required. To accomplish this, two stepper motors (once per axis) are used as shown in Figure 2.10. Such motors are capable of moving the linear positioners along its axis in two directions: forward or reverse. 


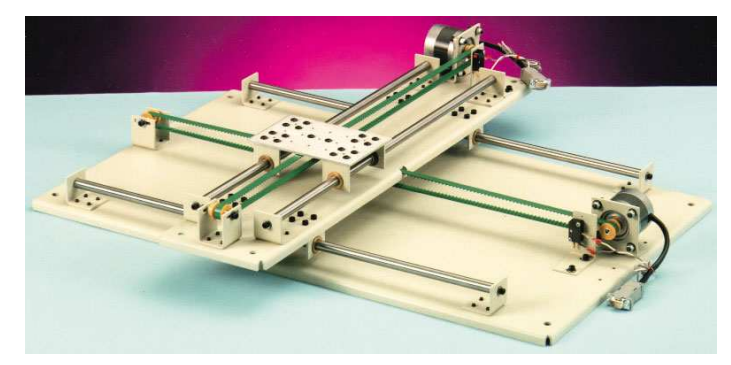

Figure 2.9. XY linear positioners [Robotics, 2011].

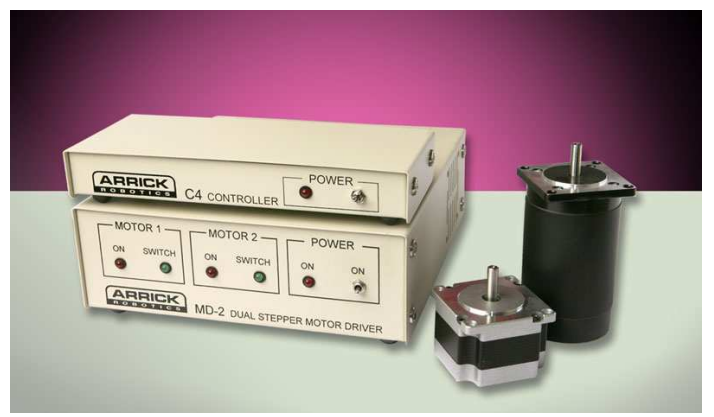

Figure 2.10. Step motors and controller [Robotics, 2011].

The step motors are controlled (and hence, the measurement procedure) by a driver, which is connected to the existing laptop via a parallel port.

\subsubsection{Software}

As mentioned before, the measurement routine of the existing channel sounder was controlled by a software application (see Appendix C, Section C.1.2). Therefore, this application should be modified to provide the user the capabilities of this new measurement method.

In Figure2.11 the configuration interface of the modified application is shown [Alcaraz Vidal, 2010]. In it, the area cover by the positioners, the number of steps and even the speed of motors can be configured. The positioners can be configured to move to one point, move along one axis with a certain number of points, or cover a $2 D$ area with a certain number of points in each dimension ( $\mathrm{X}$ and $\mathrm{Y}$ axis).

\subsubsection{Results}

In order to show the high spatial resolution of the channel sounder some indoor measurements in a room were carried out. The map of the room is shown in Figure 2.12 where the receiver and the four locations for the transmitting array are depicted. For two of them, called LOS 1 and LOS 2, transmitter and receiver are in Line-of-Sight ([LOS) while for the other two, called LOS 1 and LOS 2, transmitter and receiver are in Non Line-of-Sight (NLOS). 


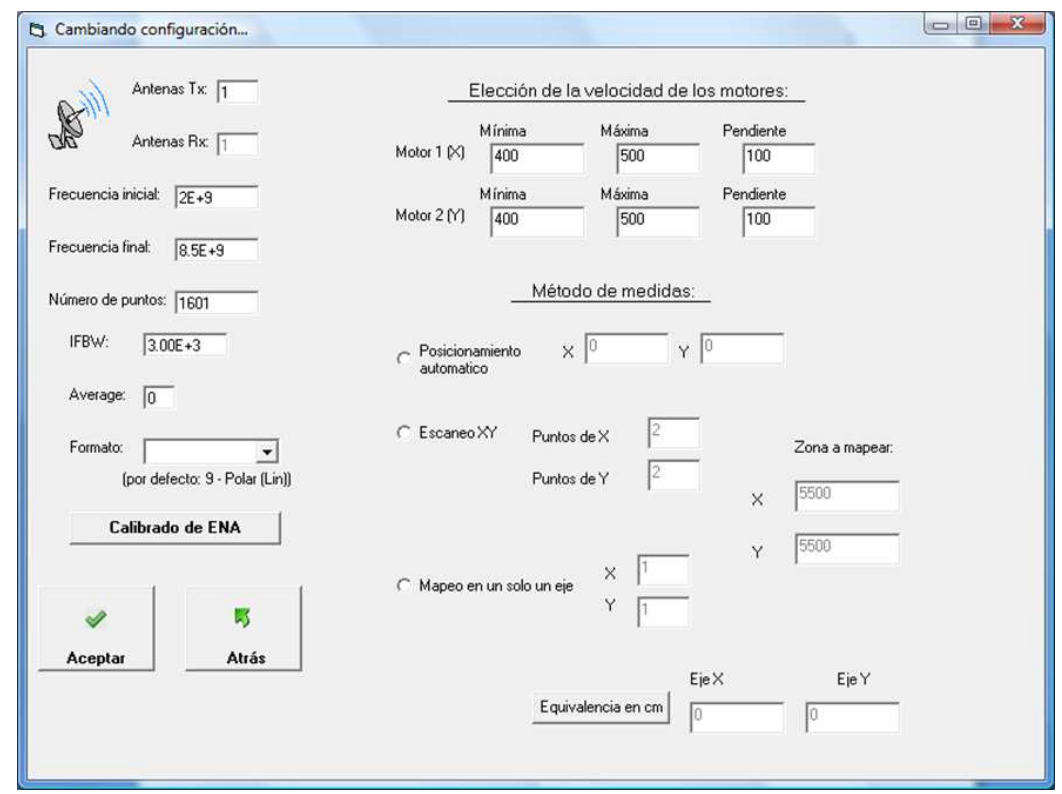

Figure 2.11. Modified interface to introduce the configuration parameters of the XY positioning system [Alcaraz Vidal, 2010].

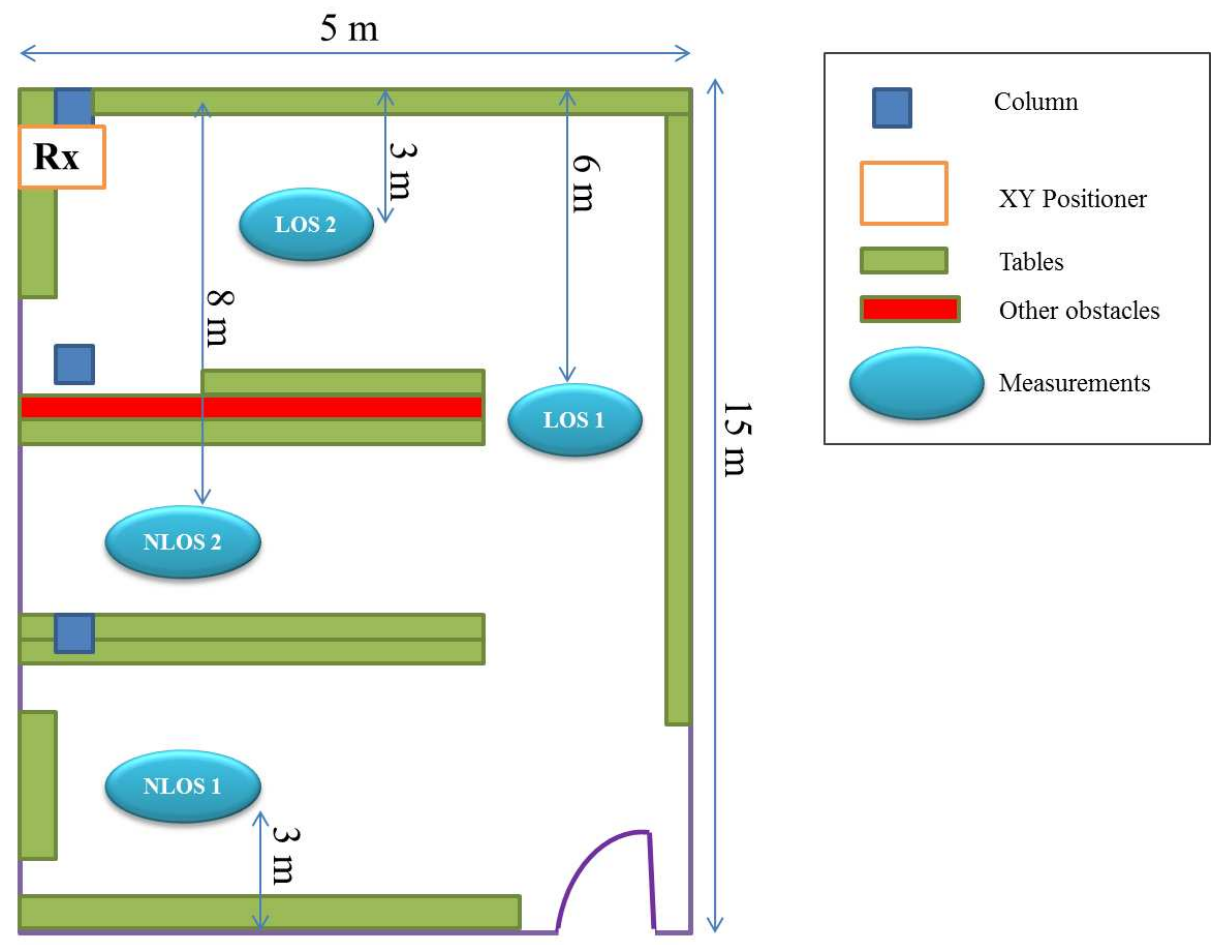

Figure 2.12. Scheme of the room where measurements were performed.

In this room, all the walls are made of plasterboard and the columns are made of concrete. There is also many computers and other equipment. The furniture is mainly made of plaster and wood as shown in Figure 2.13 


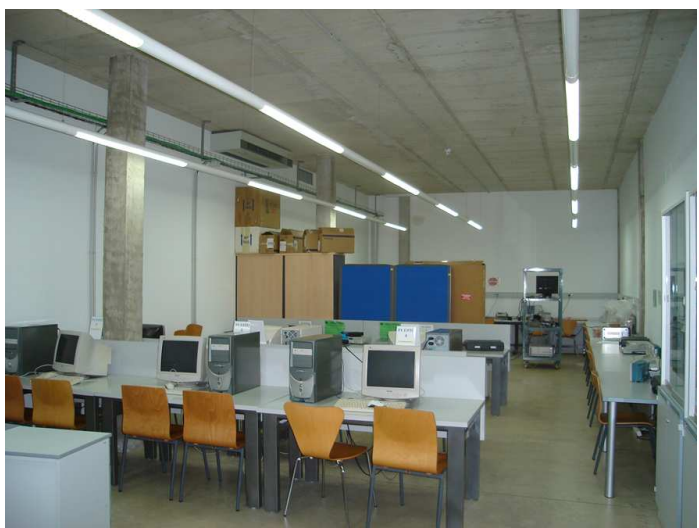

(a)

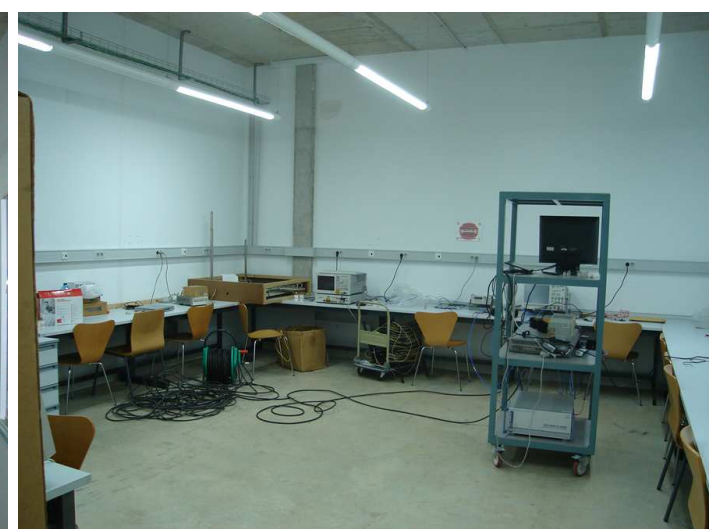

(b)

Figure 2.13. Photo of the laboratory where measurements were undertaken (a) View from the door, (b) View from the LOS 1 position.

Measurements where carried out in the $2-8 \mathrm{GHz}$ frequency band, and the configuration parameters of the channel sounder are shown in Table 2.1. The total transmitting power was $6 \mathrm{dBm}$ and the noise level was $-110 \mathrm{dBm}$. Thus, the total dynamic range was higher than $110 \mathrm{~dB}$, enough to assure a high signal to noise ratio (SNR).

\begin{tabular}{||cc||}
\hline \hline Parameter & Value \\
\hline Number of Points & 4000 \\
\hline Number of Transmitters & 4 \\
\hline Number of Receivers & 1 \\
\hline$\Delta d$ Tx & $\simeq 3 \lambda$ \\
\hline Points in X axis & 45 \\
\hline Points in Y axis & 45 \\
\hline Spatial Resolution $(\Delta x=\Delta y)$ & $1.5625 \mathrm{~cm} \simeq \lambda / 2$ \\
\hline
\end{tabular}

Table 2.1. Configuration parameters of the frequency-domain channel sounder.

To evaluate the performance of single antenna parameters in a high-resolution space domain, the Root Mean Square (RMS) delay spread (see Section B.5.2) for each position on the XY board, $(x, y)$, and for each transmitting antenna, $m$, has been computed as:

$$
\sigma_{\tau}(x, y)=E_{m}\left\{\sqrt{\frac{\sum_{k} P_{x, y, m}\left(\tau_{k}\right) \tau_{k}^{2}}{\sum_{k} P_{x, y, m}\left(\tau_{k}\right)}-\left(\frac{\sum_{k} P_{x, y, m}\left(\tau_{k}\right) \tau_{k}}{\sum_{k} P_{x, y, m}\left(\tau_{k}\right)}\right)^{2}}\right\}
$$

where $E_{m}\{\}$ is the expectation operator over all transmitting antenna elements, and denotes $x$ the position along the $\mathrm{X}$ axis, and $\mathrm{y}$ along the $\mathrm{Y}$ axis. Results are shown in Figure 2.14. 


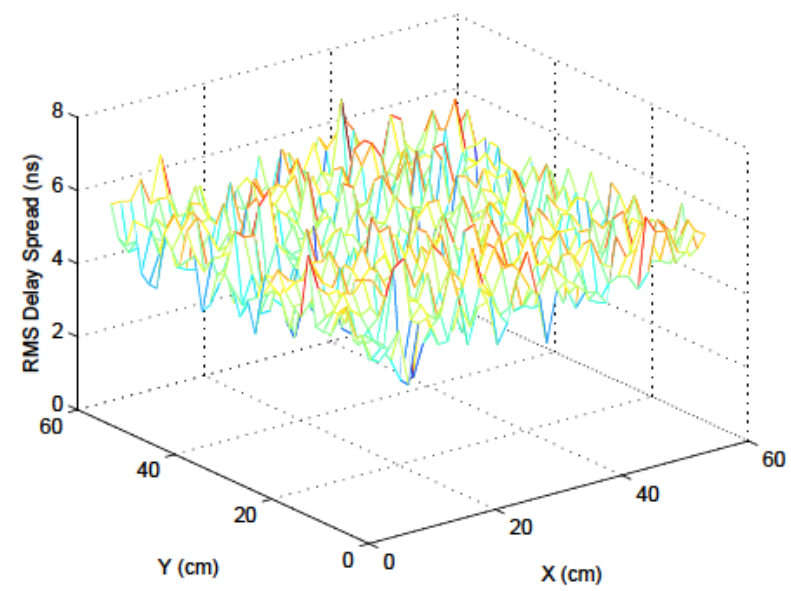

(a)

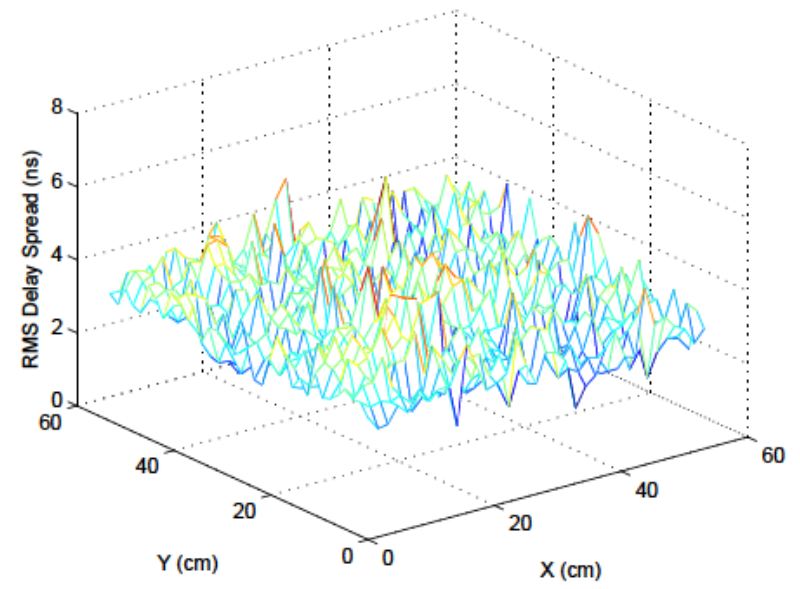

(b)

Figure 2.14. Mean RMS delay spread when the transmitter is located at (a) LOS 1 and (b) LOS 2.

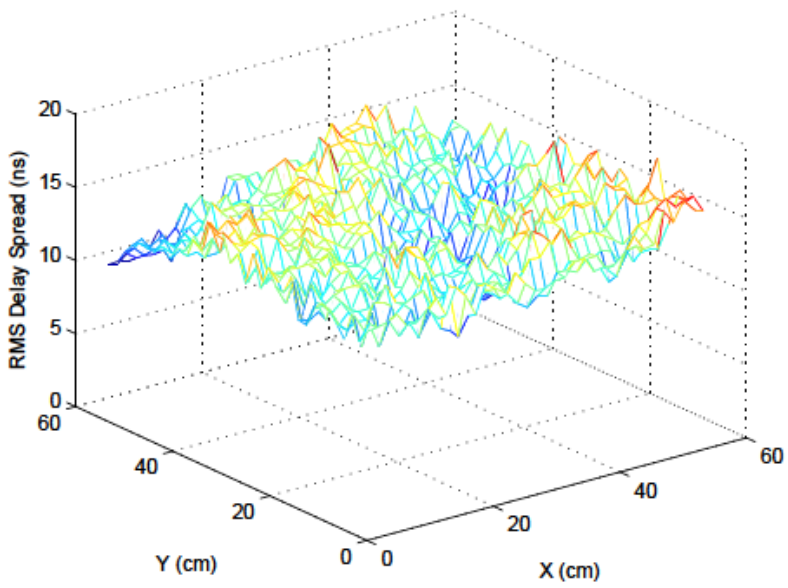

(a)

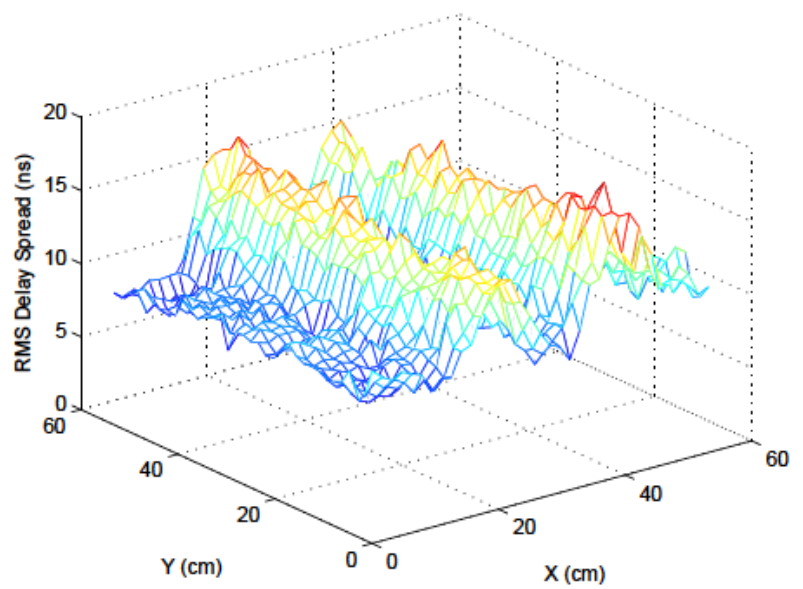

(b)

Figure 2.15. Mean RMS delay spread when the transmitter is located at (a) NLOS1 and (b) NLOS2.

Under Line-of-Sight (LoS positions) conditions, one can observe how the RMS delay spread varies between $3 \mathrm{~ns}$ and $8 \mathrm{~ns}$ within an area of $60 \times 60 \mathrm{~cm}^{2}$. This effect is due to multipath propagation in this area, which gives rise to the small scale fading (see Appendix B). For NLoS positions, the transmitter is in Non Line-of-Sight with the receiver and next to a column as shown in Figure2.12 In both cases, the RMS delay spread is considerably higher than in the case of LoS positions and varies between 10 to 20 bs as shown in Figure 2.15. However, the RMS delay spread varies strongly from one point to another of the board as in the LoS case. 
The mean and the standard deviation of the RMS delay spread for each position are summarized in Table 2.2. The standard deviation of the delay dispersion is higher when considering NLoS locations than for those in NLoS.

\begin{tabular}{||ccc||}
\hline \hline TX Location & Mean RMS Delay Spread (ns) & STD RMS Delay Spread (ns) \\
\hline LOS 1 & 3.54 & 0.59 \\
\hline LOS 2 & 5.40 & 0.64 \\
\hline NLOS 1 & 11.88 & 1.10 \\
\hline NLOS 2 & 14.17 & 1.21 \\
\hline \hline
\end{tabular}

Table 2.2. Mean and standard deviation of the RMS delay spread for each position.

If each point on the $\mathrm{XY}$ board is considered as the position of one receiving element of a virtual MIMO array, the receiving complex correlation coefficient (see Appendix $\mathrm{A}$ ) can be computed and the channel correlation can be evaluated with a high precision. The complex correlation coefficient at the frequency $f$ for the $k$ th time-realization between the reception antennas $p$ and $q$ can be computed as:

$$
\rho^{p, q}(f, k)=\frac{E\left[\overline{h^{p}(f, k)} \cdot \overline{h^{q}(f, k)}\right]}{\sqrt{E\left[\left.\overline{h^{p}(f, k)}\right|^{2} \cdot\left|\overline{h^{q}(f, k)}\right|^{2}\right]}}
$$

where $E[]$ is the expectation operation value for all the transmitting element antennas, and $\overline{h^{p}(f, k)}$ is the zero mean $p$ th row of the MIMO matrix, $H$.

The receiving correlation coefficients measured within the XY board at LOS 1, LOS 2, NLOS 1, NLOS 2 for each transmitting antenna are shown in Figure 2.16, Figure 2.17 Figure 2.18 and Figure 2.19 respectively. Such correlation is refereed to the central point of the XY board so that the correlation in this point is always 1.

The way in which the correlation decreases slowly for LoS positions (in Figure 2.16 and Figure 2.17 respectively) and rapidly for NLoS (Figure 2.18 and Figure 2.19) positions can be observed. The correlation distance (see Table 2.3) can be calculated as the point where correlation decreases the $50 \%$ of its maximum.

\begin{tabular}{||cc||}
\hline \hline TX Location & Correlation distance $\mathbf{( c m )}$ \\
\hline LOS 1 & 30.8 \\
\hline$L O S ~ 2$ & 26.3 \\
\hline NLOS 1 & 2.2 \\
\hline NLOS 2 & 2.2 \\
\hline \hline
\end{tabular}

Table 2.3. Correlation distance for each position of the transmitting array. 

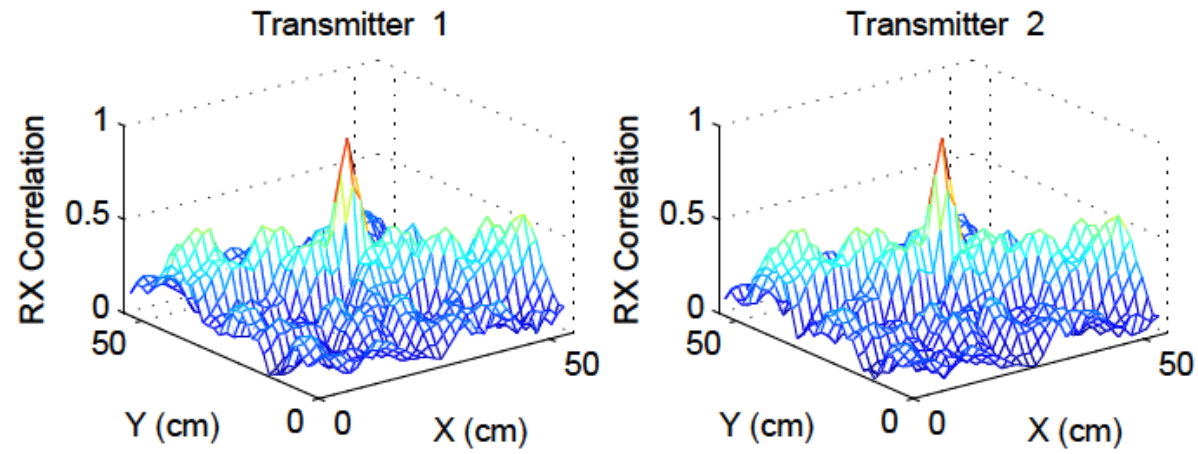

Transmitter 3

Transmitter 4
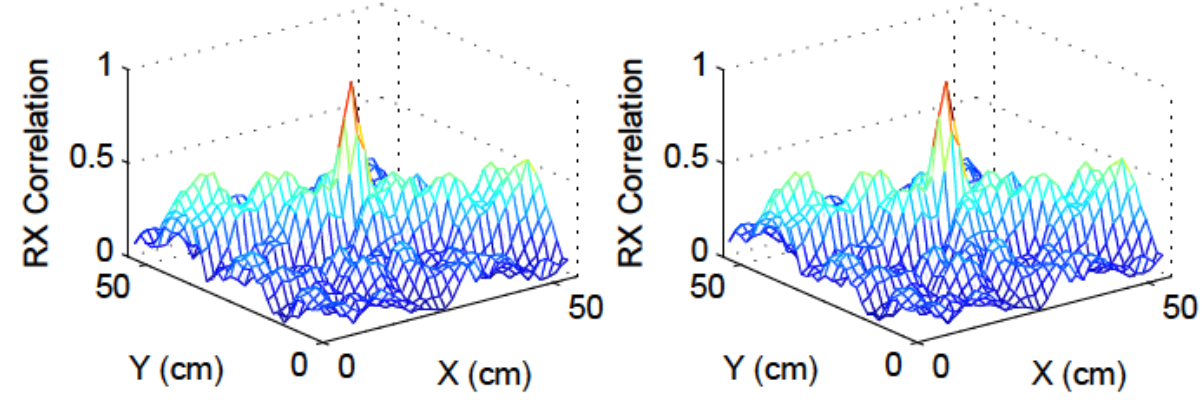

Figure 2.16. Correlation coefficient at the receiver for LOS 1 .
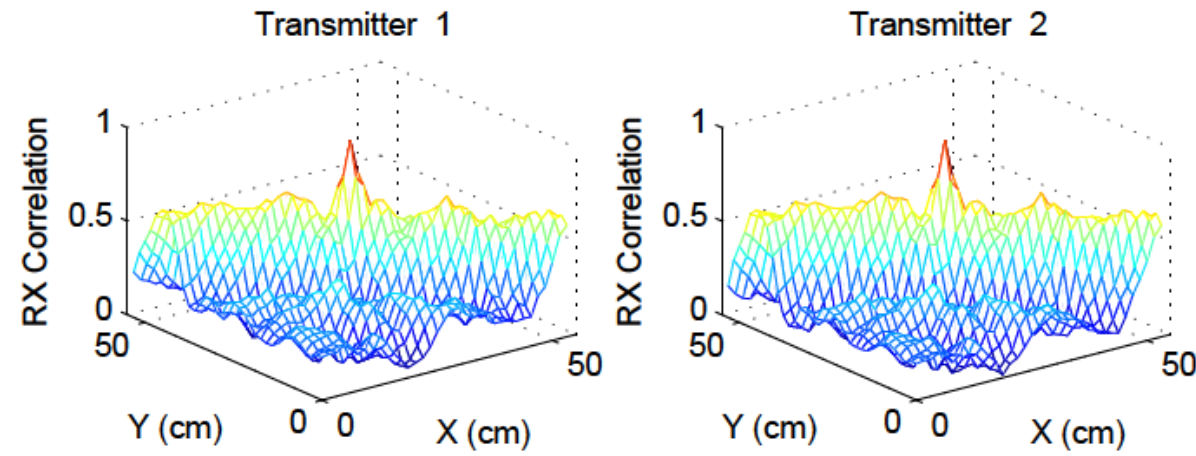

Transmitter 3

Transmitter 4
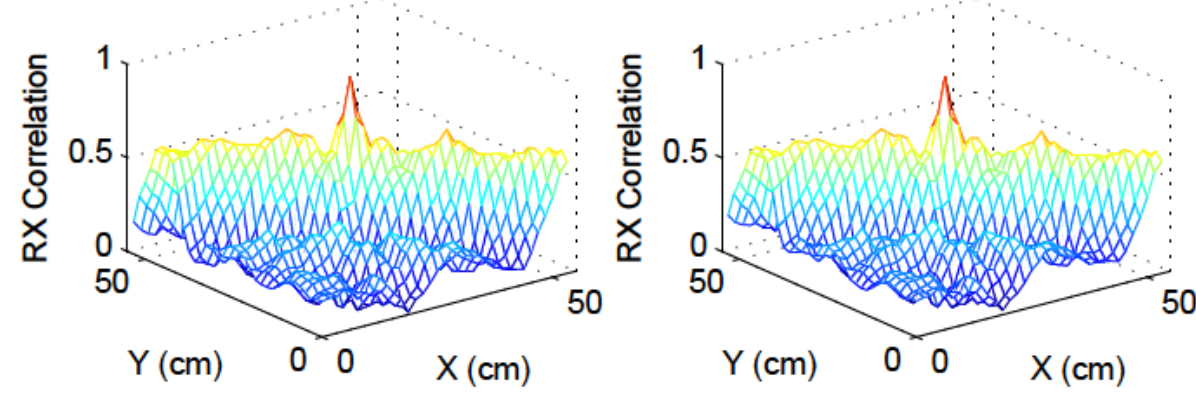

Figure 2.17. Correlation coefficient at the receiver for LOS 2 . 

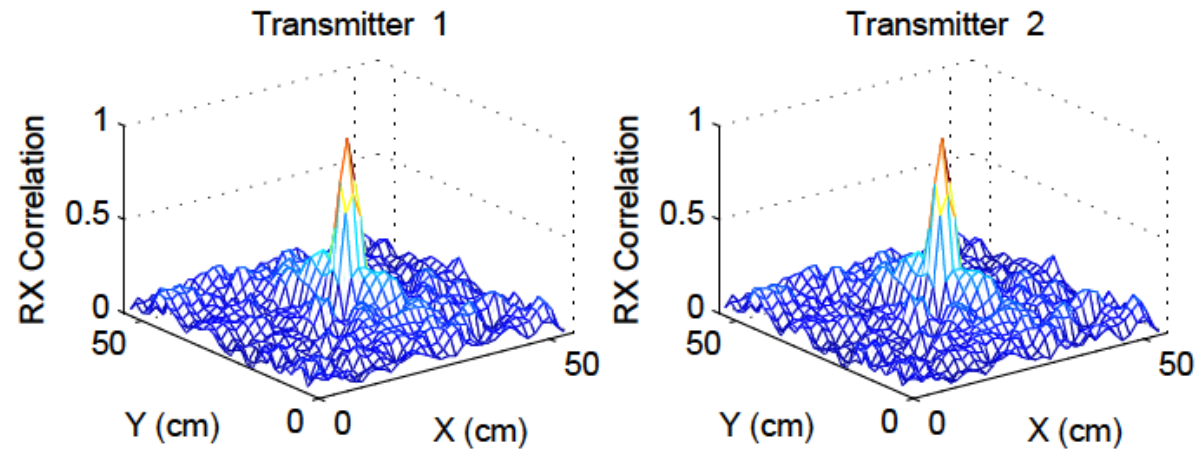

Transmitter 3

Transmitter 4
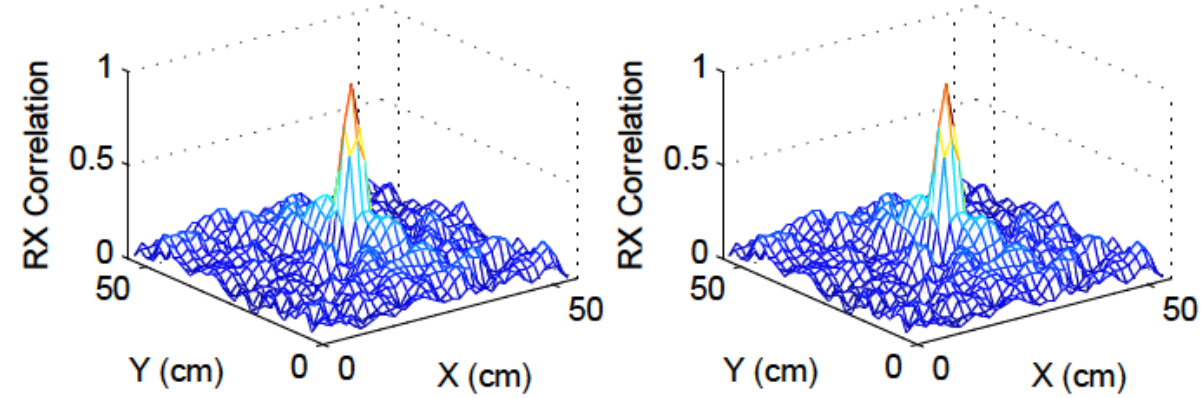

Figure 2.18. Correlation coefficient at the receiver for NLOS 1 .
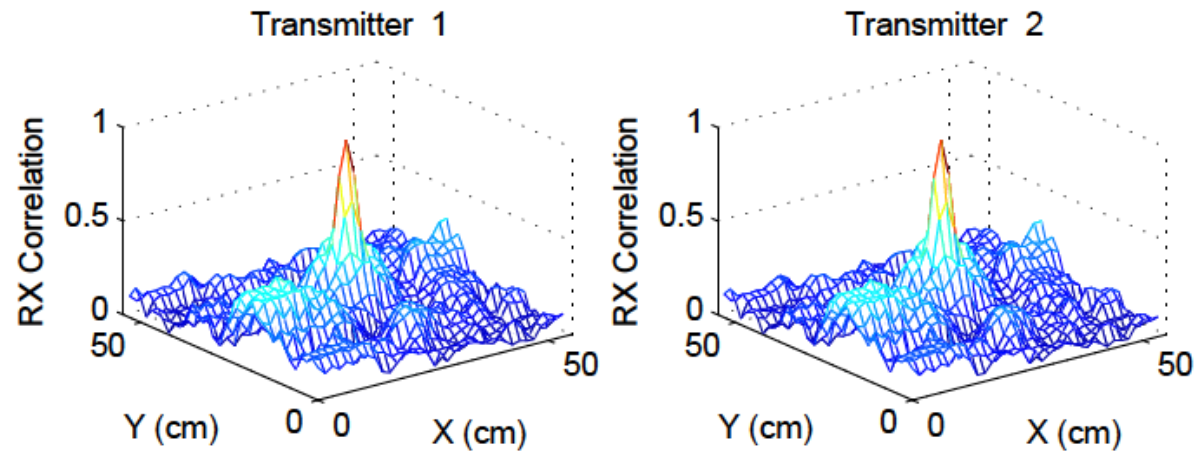

Transmitter 3

Transmitter 4
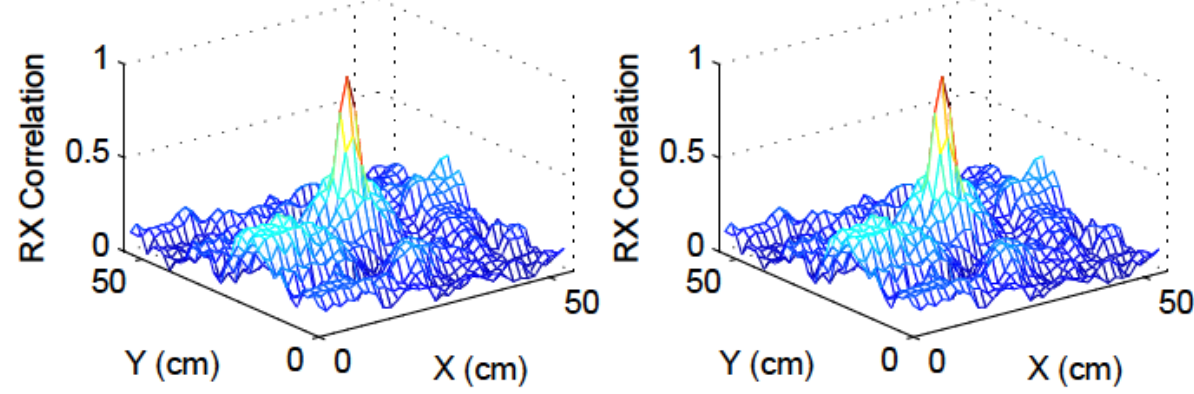

Figure 2.19. Correlation coefficient for at the receiver NLOS 2. 


\subsubsection{GIS MIMO Channel Sounding Application}

Usually, the frequency-domain MIMO channel sounder is used to undertake large measurement campaigns. Measurements are performed in several scenarios and both transmitter an receiver is located at many locations. Thus, the user should relate by hand all the locations and scenarios with the measurements stored at the MVNA. One possible solution to this problem is the integration of the measurement software application into a Geographic Information System.

A Geographic Information System (GIS), is a system designed to capture, store, manipulate, analyze, manage, and present all types of geographically referenced data. In other words, a GIS is the association of cartography, statistical analysis, and database technology. GIS applications are also tools that allow users to analyze spatial information, edit data, maps, and present the results of all these operations.

With a GIS, you can model geographic information or data in three basic ways: as a collection of discrete features in vector format, as a grid of cells with spectral or attribute data (raster), or as a set of triangulated points modeling a surface [Zeiler, 1999] as explained below. Choosing and adequate data representation depends on the requirements of the application or the analysis to carry out.

In this thesis, the GIS used has been ArcGIS Desktop 9.1 from the company ESRI, available at the Group of Mobile Communications (SiCoMo). ArcGIS Desktop is a product that performs advanced spatial analysis, model operational processes, and visualize results on professional-quality maps. It is an integrated suite of desktop applications for Windows such as ArcMap, ArcCatalog, ArcToolbox and ArcGlobe.

The application that controls the measurement routine (shown in Figure 2.7) has been integrated into ArcMap, which is the central application in ArcGIS Desktop [ESRI, 2005]. With ArcMap, the user can carry out tasks based on maps management. It includes cartography, map analysis and editing. A more detailed explanation about Geographic Information Systems, data representation, ArcGIS and ArcMap is available in AppendixD.

In the following section the integration of the measurement software with the GIS is detailed.

\subsubsection{Implementation}

The modified software application is integrated with ArcMap as an user-defined toolbar. This toolbar provides two main groups of functions called Data Base and Measurements and it is shown in Figure 2.20, These features are explained next.

Moreover, the toolbar provides some other functions such as Help, Draw by Hand on the map, or Clean Screen. 


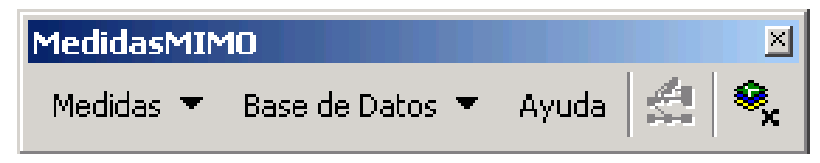

Figure 2.20. Application toolbar.

\section{A. Data Base}

The Data Base tools are used to define the locations where transmitter and/or receivers will be located. These definitions must be done before the measurement campaign. To achieve this goal, it is necessary to have a map of the measurement scenario. Such maps can be created with many design software such as, Autocad.

Firstly, locations are defined directly over the map or introducing its coordinates. All the locations are stored in the location Data Base and can be identified by its name and coordinates. Each location can be opened, copied and deleted.

Secondly, once the locations of the measurement campaign have been created, one can define the measurement route. A measurement route is defined as the group of locations used in the measurement campaign where the transmitter will be located in only one position, while the receiver can be located in many positions. In other words, in a measurement route the transmitter array will be placed at a fixed location, while the receiver array can be placed at different location. If the transmitter's location is changed, a new measurement route should be created.

Once, the measurement locations have been established, the measurement campaign can be started. The measurement procedure is explained next.

\section{B. Measurements}

The Measurements tools are used to on the one hand, relate a previously created measurement route with real measurements to be done, and on the other hand, to carry out the calibration procedure and the measurement routine. The application also guides the user through all this steps.

After assigning a name to measurements to be done, the first step is select the measurement route (from all the measurement routes created and stored in the data base) where measurements will be undertaken. In Figure 2.21, the measurement scenario and the measurement route are shown.

Secondly, the measurement configuration parameters such the number of transmitting and receiving antennas, as the frequency band, the number of points, and the format (as shown in Figure 2.7b, should be introduced.

Next, the software guides the user through the calibration procedure, which is now automatic. 


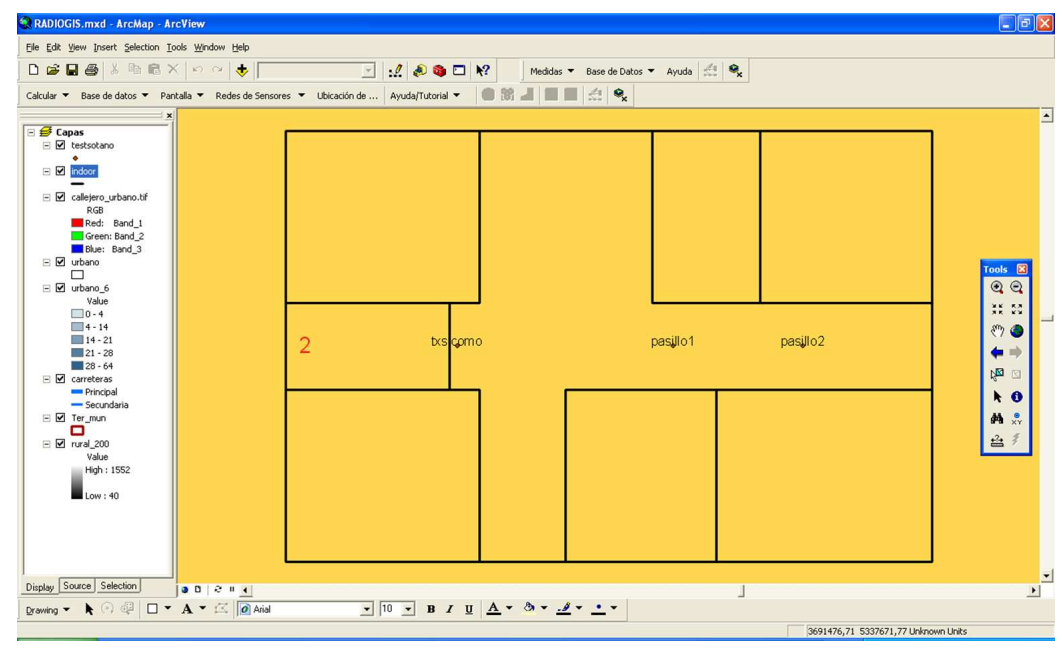

Figure 2.21. Measurement scenario in the GIS.

This procedure was usually long and tedious, and its complexity increased with the number of antennas of the MIMO system. This feature was not included in the first version of the channel sounder (explained at the beginning of Section 2.2). The software application inform the user which pair of antennas should connect in order to calibrate (see Appendix C, Section C.2.1) such pair and eliminate the effects of cables, connectors and so on from the final measurements. This procedure is repeated for all the pairs of antennas and the calibration state is stored into the vector network analyzer.

Finally, measurements are carried out along the measurement route indicated in the first step of the measurement process. The software guides all these procedure by informing the user where the receiver (the transmitter is considered to be fixed) should be located.

Measurements are stored in the MVNA into a folder whose name is the name that was assigned to the measurements in the first step of the measurement procedure. Inside this folder, there is the calibration state file and as many subfolders as receivers in the measurement route are, as shown in Figure 2.22. In each receiver folder the measured data at this location is stored.

With this software the spatial and geographic information of the measurement route is directly related with the measurement data stored by the MVNA. Thus, measurements can be analyzed into the GIS and compared with theoretical results. Furthermore, the features provided by the GIS in data analysis provide the capability of performing more detailed analyses of the measurements.

\subsection{Time-Domain MIMO Channel Sounder}

In the Appendix $\mathrm{C}$ a frequency-domain channel sounder based on a MVNA existing in the group SiCoMo was described [İbernón-Fernández et al., 2008]. Furthermore, along Section[2.2 two 


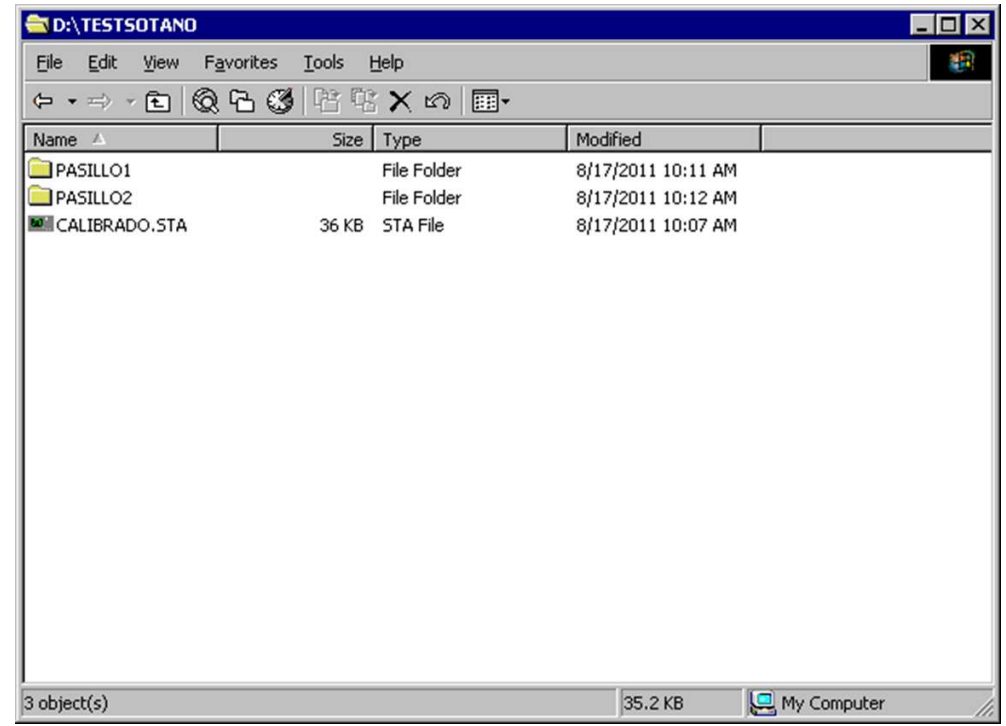

Figure 2.22. Folders created by the application in the MVNA hard disk.

modifications of this channel sounder were proposed with the aim of incorporate new features into this classical measurement system: high spatial resolution and georeferencing capabilities. In all these implementations the multiplexation of the frequency-domain signal between all antennas is a fully switched scheme (TDM). The main drawback of this scheme is the huge amount of time needed to complete a $N \times M$ MIMO measurement, which is of the order of several seconds. During this large time the channel must remain invariant so measurements of time-variant MIMO wideband channels can be erroneous.

For this reason, this section is devoted to the development of a time-domain wideband MIMO channel sounder. Such measurement system is based on the spread spectrum sliding correlator channel sounding scheme presented on Section 2.1.1, where sequences with special autocorrelation properties as $\mathrm{PN}$ sequences, are transmitted and received by a digital oscilloscope. The received signals are then postprocesed to obtain the channel impulse response [Ciccognani et al., 2005]. However, this scheme is suitable only for SISO systems. For MIMO systems, not only should have the sequences good autocorrelation properties, but also adequate crosscorrelation properties. Therefore, it is also necessary to find sequences that meet at the same time appropriate autocorrelation and crosscorrelation properties [Choi et al., 2006b], [Wonsop et al., 2007].

Once the sequences are chosen, the channel sounder is implemented. To accomplish this, commercial multipurpose equipment, which is present in most RF research centers, is used: a baseband generator, an I/Q modulator, and a digital storage oscilloscope. The main difference with existing time-domain MIMO channel sounders such as the well-known RUSK [GmbH, 2011] is on the one hand the overall price of the channel sounder (in the case of the RUSK several hundred thousand euros); and in the other hand, the capability of re-utilize the equipment for other different applications. 
The equipment used in this time-domain channel sounder is connected as depicted in Figure 2.23 and configured as described in the following sections to achieve the correct operation of the channel sounder. Furthermore, a software application to control and automatize all the measurement and acquisition procedure is developed.

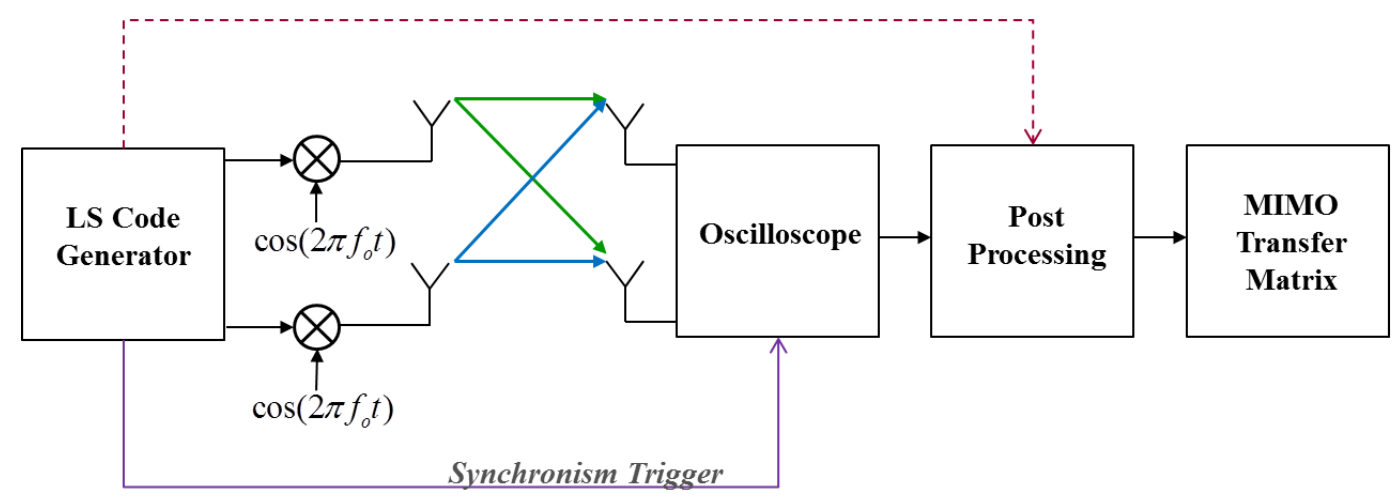

Figure 2.23. Block Diagram of the time-domain MIMO channel sounder.

Finally, measurement campaigns should be undertaken with the system in frequency domain and the system in time domain, in order to evaluate the accuracy and features of the new measurement system. The aim is to compare the results provided by both systems.

\subsubsection{Loosely-Synchronous Codes}

As mentioned before, PN sequences are suitable for SISO time-domain channel sounding but not for MIMO implementation due to its bad crosscorrelation properties. In this case, it is necessary to find a set of sequences with impulsive autocorrelation functions and zero crosscorrelation functions. This kind of sequences, called LAS (Large Area Synchronized) [Stanczak et al., 2001] codes were proposed in the early 2000 for Quasi-Synchronous Code Division Multiple Access (QS-CDMA) systems [Fan, 2004] in third generation (3G) and fourth generation (4G) wireless communications. Such sequences result from a combination of Large Area (LA) codes and pulse compressing Loosely Synchronous (LS) code. In Kim et al., 2008 the performances of a simulated MIMO channel sounder using LS, and other codes with similar properties such as Kasami and Chaotic sequences are analyzed. This study concludes that LS sequences are the most suitable for a low number of transmitting antennas.

The MIMO $2 \times 2$ channel sounder developed is based on the transmission of two LS codes instead of PN-sequences. LS codes (shown in Figure 2.24) are based on Golay complementary codes [Golay, 1961], where additional zeros, $W_{0}$, are inserted between a complementary pair in order to create an area called Interference Free Window (IFW). Thus, the autocorrelation and crosscorrelation properties required to avoid interference between transmitting antennas are achieved as shown in Figure 2.25. 

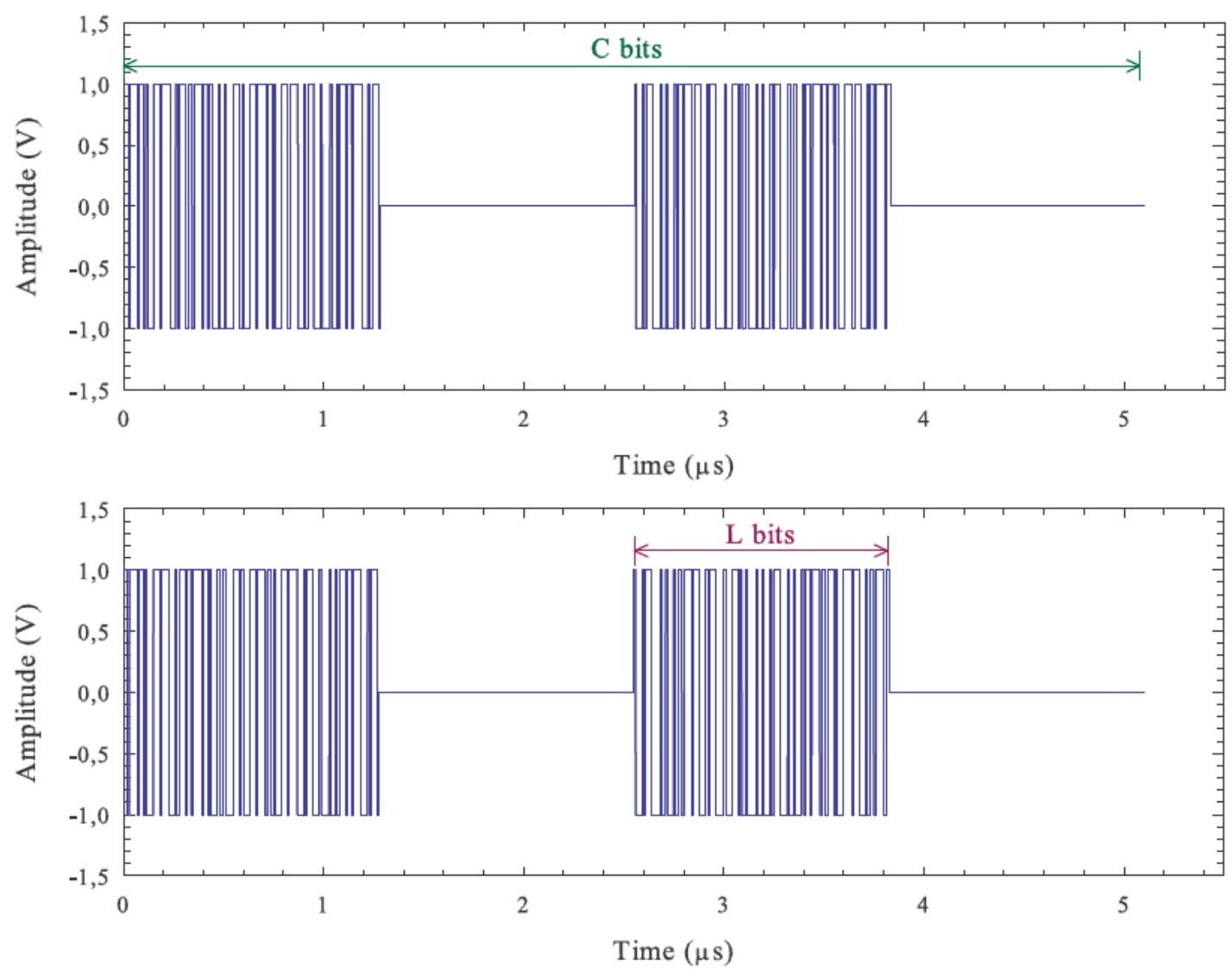

Figure 2.24. LS codes used in the channel sounder.

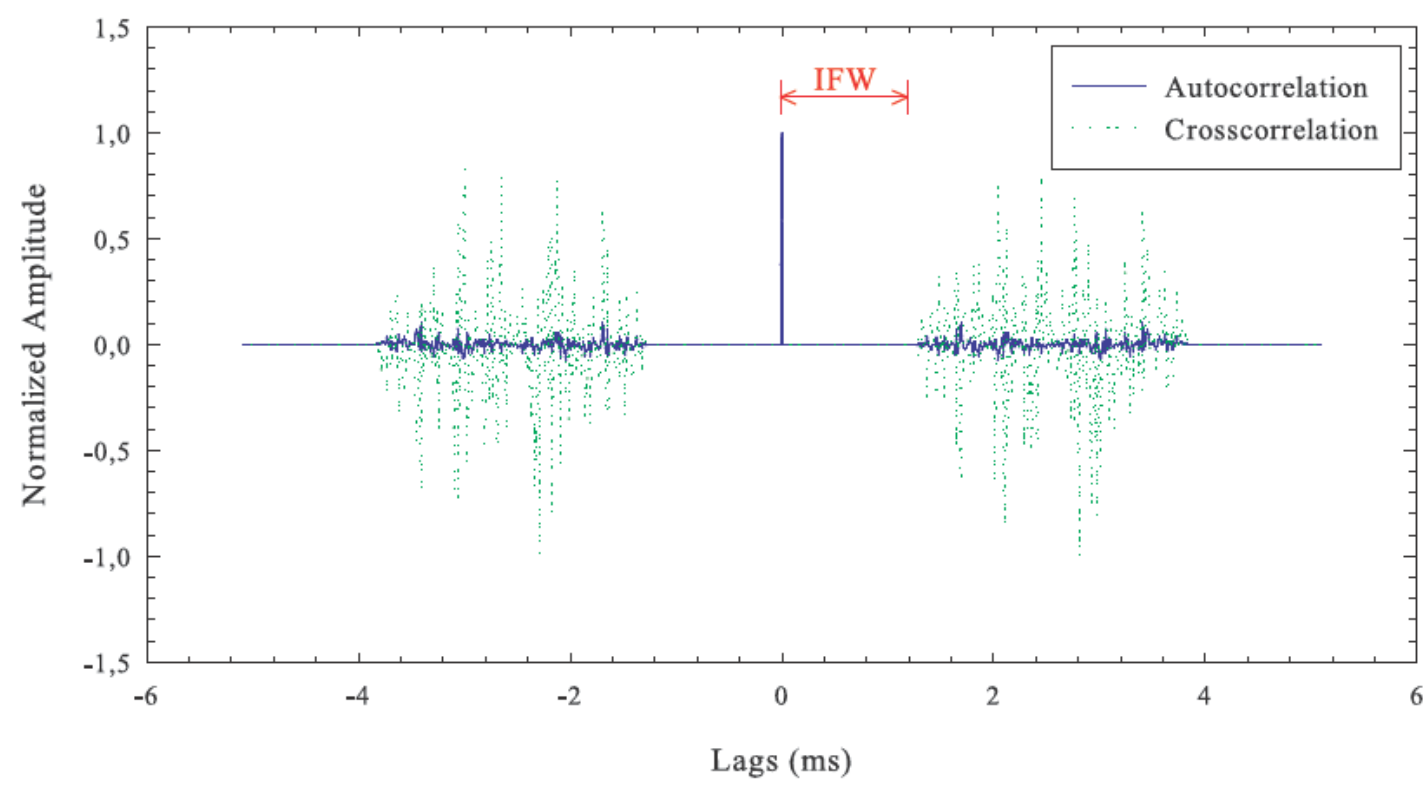

Figure 2.25. Autocorrelation and crosscorrelation of the LS codes. 
Nevertheless, it should be taken into account that the codes are shaped just by square pulses as shown in Figure 2.24. The main drawback of this implementation is the nearly infinite bandwidth of the codes. Since a demodulation procedure is required in reception, as described in Section 2.3.2.5, it is necessary to find a pulse shape which provides a narrower frequency response. To accomplish this, a rised-cosine pulse shaping was carried out. The rolloff factor is designed to 0.8 in order to obtain a $-3 d B$ bandwidth at $f_{\text {chip }} / 2$, and a whole bandwidth of approximately $f_{\text {chip }}$ as shown in Figure 2.26 for $f_{\text {chip }}=100 \mathrm{MHz}, f_{\text {chip }}$ being the frequency chip of the code.

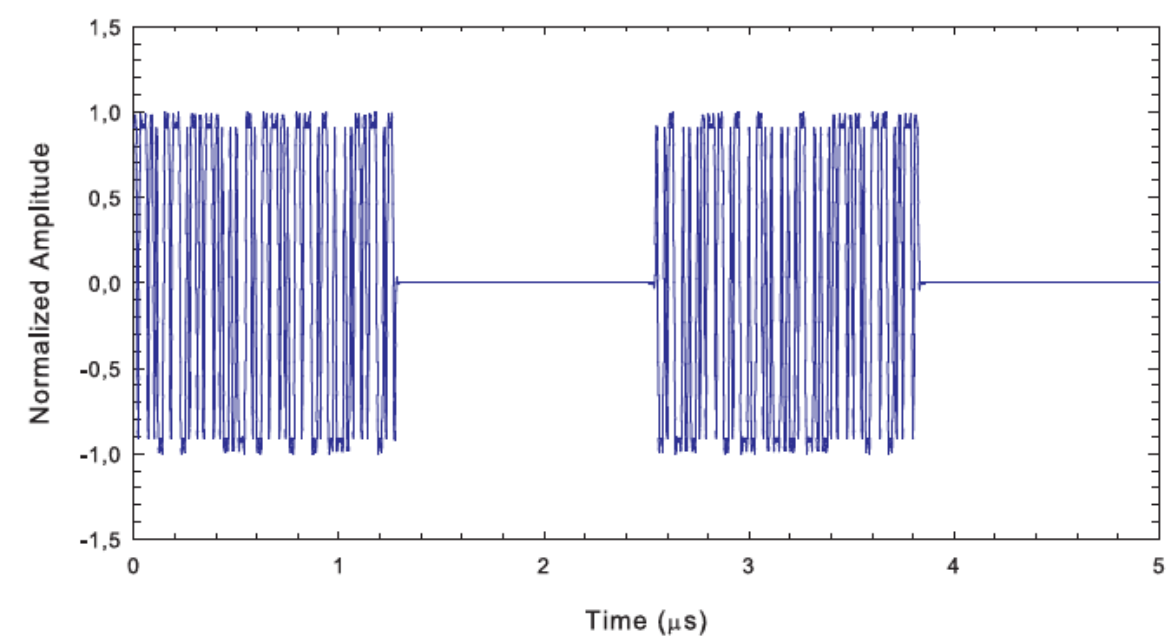

(a)

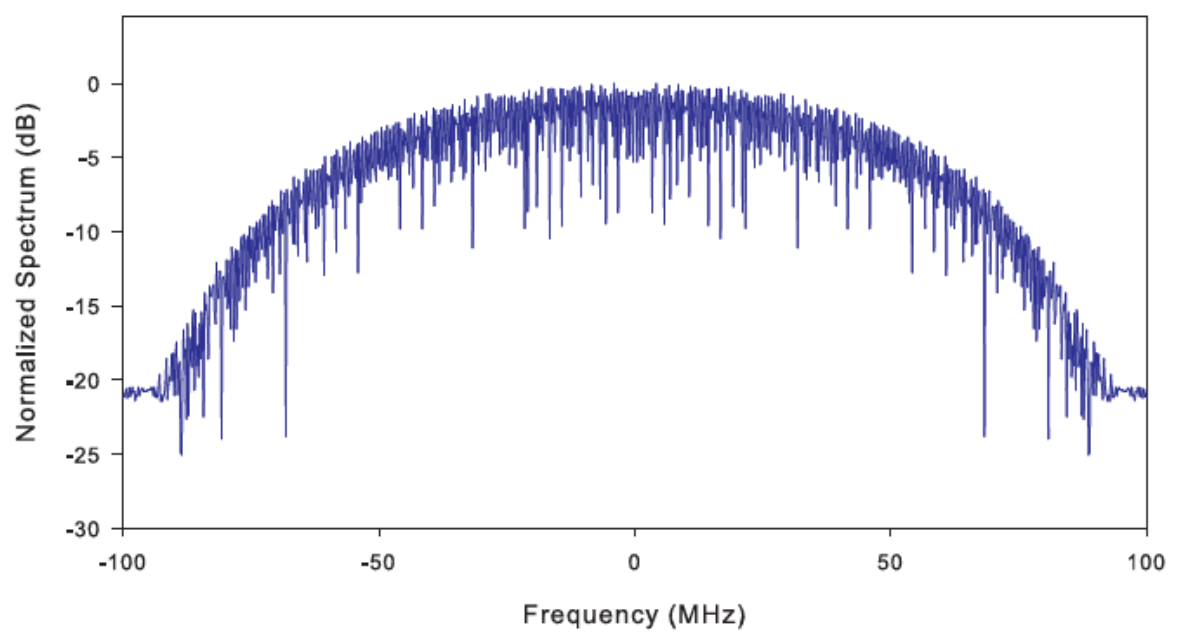

(b)

Figure 2.26. (b) LS code after rised-cosine pulse shaping in time domain (b) Normalized Spectrum of the code. 


\subsubsection{Implementation}

The time-domain channel sounder is mainly based on the transmission on LS codes instead of PN-sequences. Assigning one LS code to each transmitting antenna it is possible to transmit with all the elements at the same time so the the wireless MIMO channel can be measured in real-time. Figure 2.27 depicts the time-domain $2 \times 2 \mathrm{MIMO}$ channel sounder, that is based on a baseband pulse generator, an I/Q modulator and an oscilloscope. The different devices that compose the equipment system and how they work are described in Section 2.3.2.1.

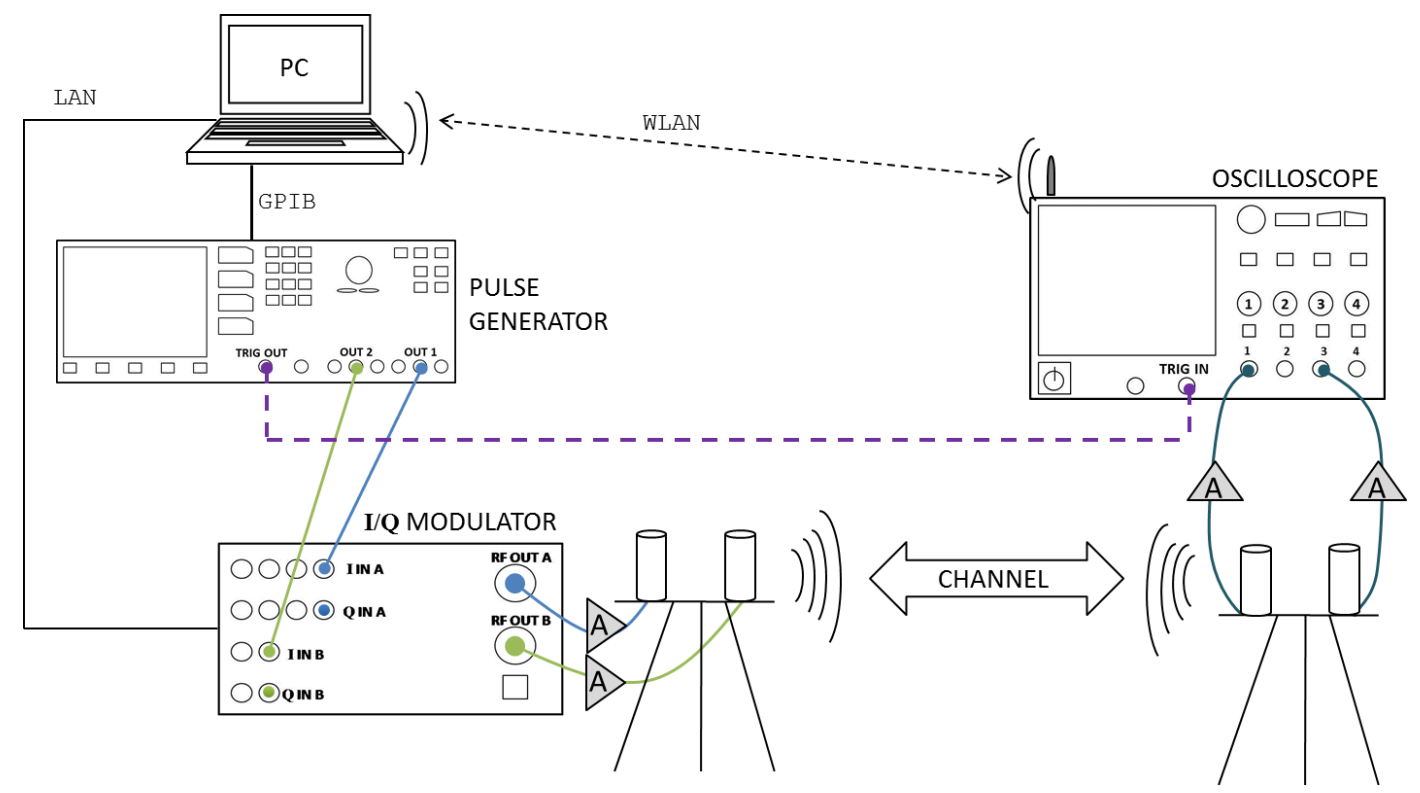

Figure 2.27. Time-domain MIMO channel sounder.

After the assembly and connection of all the equipment, the measurement procedure is controlled by a software application, described in Section 2.3.2.2 and shown in Figure 2.35. This application configures all the devices with the parameters required and controls the measurement routine. When the measurement routine finishes, the data stored in the oscilloscope is post-processed in MATLAB (see Section 2.3.2.5) to extract wideband MIMO parameters. This measurement procedure is depicted in Figure 2.28 and detailed in the following sections.

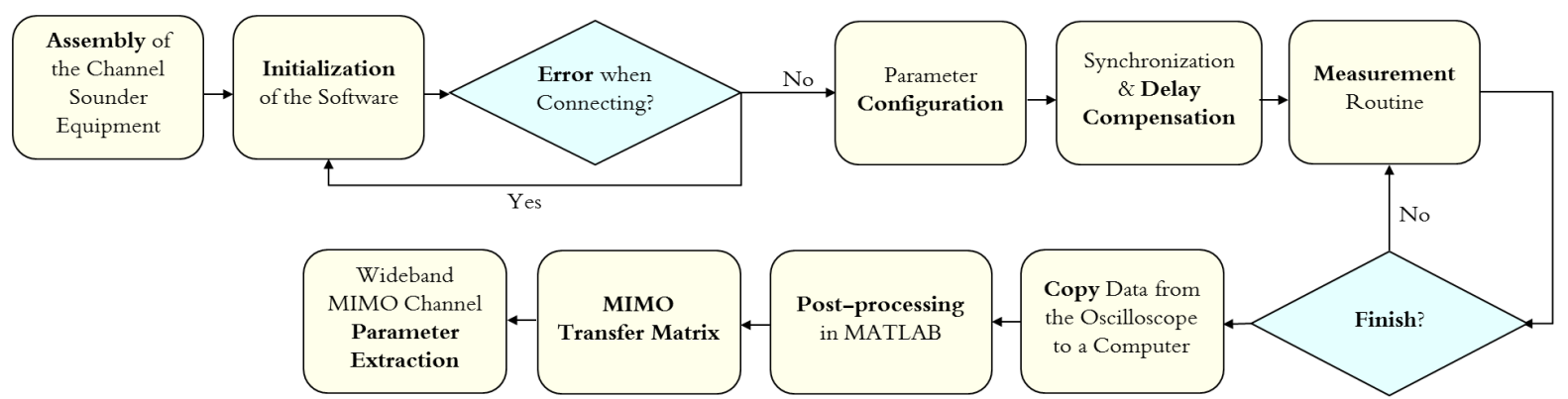

Figure 2.28. Block diagram of the measurement procedure. 


\subsubsection{Hardware Equipment}

\section{A. Baseband Signal Generator}

Firstly, the LS codes, $c_{1}(t)$ and $c_{2}(t)$, generated in MATLAB are downloaded and stored in the Agilent 81550A Pulse Function Arbitrary Noise Generator, shown in Figure 2.29. This device can generate pulse, sine, square, ramp, noise and arbitrary waveforms with many modulation capabilities. It can also generate arbitrary signals with a bandwidth of up to $120 \mathrm{MHz}$ in its two channels, providing four internal waveform memories, where the LS codes are stored. Each memory has a capacity of 512 ksamples.

Regarding trigger signals, it is equipped with a trigger input as well as a trigger output for each of its two channel outputs. Furthermore, both channel outputs can be coupled in order to replicate the frequency, trigger mode, waveform type and advanced mode from one channel to the other one.

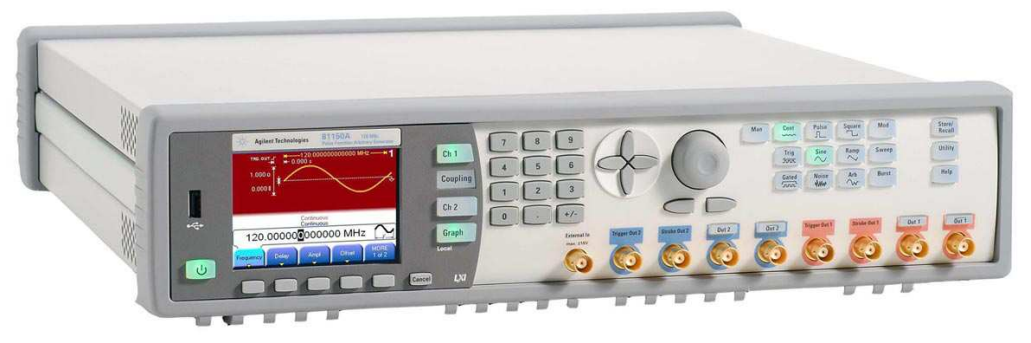

Figure 2.29. Base band signal generator.

Since each LS sequence has a total length of $C$ bits, and each complementary pair has $L$ bits, for a given frequency chip, $f_{c h i p}$, the $81150 \mathrm{~A}$ generator repeats the whole LS sequence with a frequency

$$
f_{r}(H z)=\frac{f_{\text {chip }}}{C}
$$

It should be taken into account that the two sequences should be coupled in order to maintain the alignment between sequences. Thus, the good autocorrelation and crosscorrelation properties of the LS codes are preserved.

\section{B. Modulator}

The two LS sequences, $c_{1}(t)$ and $c_{2}(t)$, generated by the baseband generator are connected to the in-phase (I) inputs of the two channels I/Q Rhode\&Schwarz SMATE200A Vector Signal Generator (Figure 2.30) which can works between $100 \mathrm{kHz}$ up to $6 \mathrm{GHz}$ within a bandwidth of $200 \mathrm{MHz}$.

The quadrature-phase $(\mathrm{Q})$ input of both channels is adapted so the modulator works as a carrier at the central frequency of the bandwidth under measurement. This carrier frequency (central 

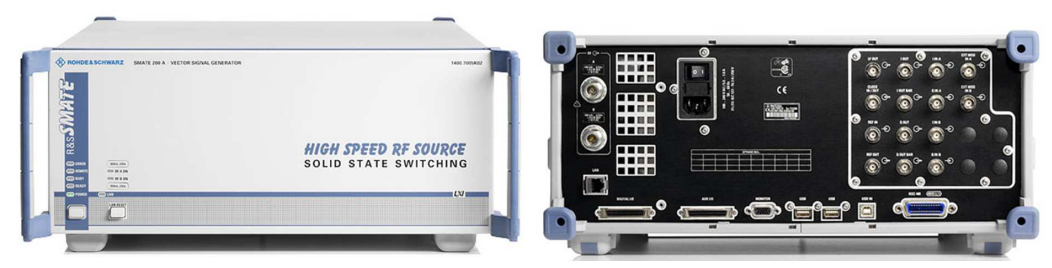

Figure 2.30. Rohde\&Schwarz I/Q Modulator.

frequency of the frequency band to be sounded), $f_{0}$, as well as the output power (in $\mathrm{dBm}$ ) can be adjusted with a high precision for both channels. Thus, the modulated and transmitted signals are

$$
\begin{aligned}
& s_{T X_{1}}(t)=c_{1}(t) \cos \left(2 \pi f_{0} t+\phi_{1}\right) \\
& s_{T X_{2}}(t)=c_{2}(t) \cos \left(2 \pi f_{0} t+\phi_{2}\right)
\end{aligned}
$$

these signals are amplified (see Section 2.3.2.1,E) before being transmitted by the antennas (see Section 2.3.2.1.F).

\section{Oscilloscope}

In the reception side, the signal detected by each antenna is filtered and then amplified (see Section 2.3.2.1,E). After, the signals are acquired and stored by the Agilent Infiniium 90804A highperformance oscilloscope, shown in Figure2.31. This equipment can acquire signals of up to $8 \mathrm{GHz}$ sampling them up to $40 \mathrm{Gsa} / \mathrm{s}$ in its four channels. However, in order to minimize the size of the acquisition, sampling frequency is limited depending on the maximum frequency to measure.

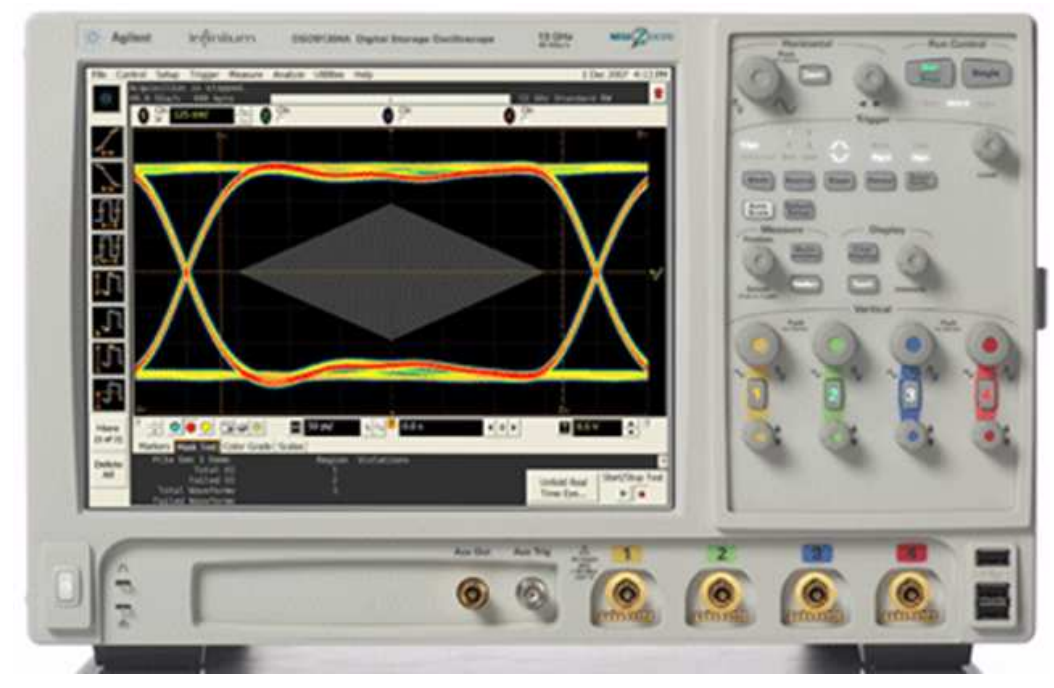

Figure 2.31. Agilent 90804A oscilloscope.

The vertical resolution of the oscilloscope is 8 bits (256 voltage values) in its 8 vertical divisions. It should be taken into account that the received signal should fit in the whole vertical range in order to minimize the quantification noise. Thus, the received signal can be acquired correctly. 
Furthermore, the weakest signal that the oscilloscope can detect depends on the vertical scale used to acquire the signal, so the dynamic range of the channel sounder varies with this parameter (see Section 2.3.2.4). Therefore, the measurement is affected by the vertical scale chosen so this parameter should be controlled in order to measure accurately the received signal.

The horizontal scale depends directly on the acquisition time, $T_{a d q}$, which is equal to the the duration of the whole code

$$
T_{a d q}=\frac{1}{f_{r}}=\frac{C}{f_{\text {chip }}}
$$

plus an extra $10 \%$ to allow all the multipath components being received and thus, avoid inter-symbol interference between consecutive received sequences.

\section{Computer}

A Visual Basic application (described in Section 2.3.2.2) has been developed to control and automatize all the measurement and acquisition procedure. This application is executed in a PC that controls all the elements of the system via Local Area Network (LAN), Wireless LAN (WLAN) and General Purpose Interface Bus (GPIB) interfaces, as shown in Figure 2.27.

\section{E. Filters and Amplifiers}

As mentioned before and shown in Figure 2.27, the modulated signals are amplified before being transmitted by the antennas. In the same way, the signal detected by the receiving antennas is filtered and amplified before being acquired by the oscilloscope.

On the one hand, to amplify the transmitted and received signals, four identical Minicircuits ZVE-8G+ amplifiers (Figure 2.32a), whose mean gain is $30 \mathrm{~dB}$, are used. The DC voltage operation is $12 \mathrm{~V}$, and the maximum input power is $20 \mathrm{dBm}$. Hence, in the transmitting side, the maximum output power of the R\&S SMATE200A must be lower to this value.

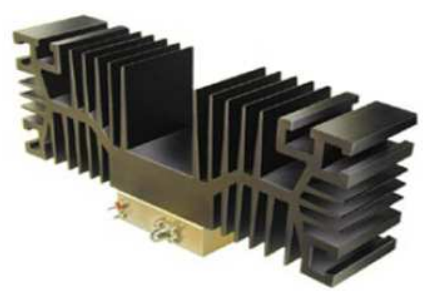

(a)

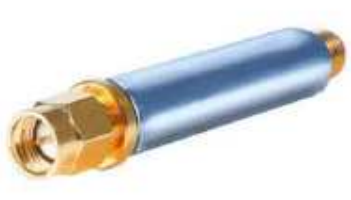

(b)

Figure 2.32. (a) ZVE-8G+ amplifier, (b) VBFZ passband filter, both from MiniCircuits [MiniCircuits, ].

On the other hand, to filter the signal received by the antennas, two MiniCircuits VBFZ coaxial passband filters (Figure 2.32b) are used. The central frequency of such filters depends on the central 
frequency of the band under analysis. Due to the low cost of each filter and that its central frequency $\left(f_{0}\right)$ is fixed, different models of this filter are used in measurements. The passband varies with the model used from approximately $600 \mathrm{MHz}$ up to $1.3 \mathrm{GHz}$, and the insertion losses in the pass band are between 2 and $4 \mathrm{~dB}$ for all the models as summarized in Table 2.4 .

\begin{tabular}{||ccc||}
\hline \hline Model & Band & Insertion Losses \\
\hline VBF-2340+ & $2020-2660 \mathrm{MHz}$ & $<2 \mathrm{~dB}$ \\
\hline VBF-2555+ & $2500-2610 \mathrm{MHz}$ & $<4 \mathrm{~dB}$ \\
\hline VBFZ-2575+ & $2350-2800 \mathrm{MHz}$ & $<2 \mathrm{~dB}$ \\
\hline VBFZ-2575+ & $2350-2800 \mathrm{MHz}$ & $<2 \mathrm{~dB}$ \\
\hline VBFZ-3590+ & $3000-4300 \mathrm{MHz}$ & $<2 \mathrm{~dB}$ \\
\hline VBFZ-3590+ & $3000-4300 \mathrm{MHz}$ & $<2 \mathrm{~dB}$ \\
\hline VBFZ-5500+ & $4900-6200 \mathrm{MHz}$ & $<2 \mathrm{~dB}$ \\
\hline \hline
\end{tabular}

Table 2.4. Different filters used in the channel sounder.

\section{F. Antennas}

For the measurements, four omni-directional Electrometrics EM-6116 antennas (Figure 2.33) have been used. This antenna operates in the 2-10 GHz band and provides a gain of $1 \mathrm{dBi}$.

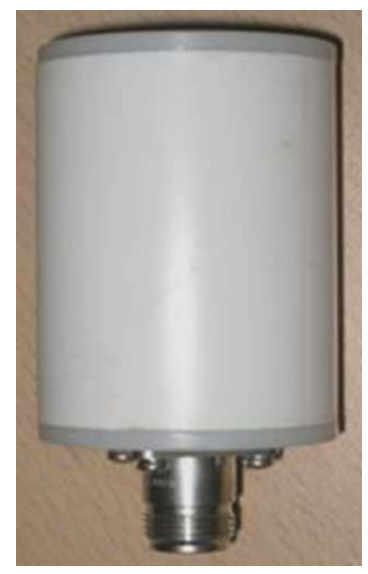

Figure 2.33. Electrometrics EM-6116 antenna.

\section{G. Cables and Connectors}

Coaxial cables of 1.5 meters long and SMA, N and BCN connectors and transitions are used to interconnect all the devices of the channel sounder as shown in Figure 2.27

\section{G. Others}

Some additional equipment is used to manage the correct operation of the channel sounder developed. An external D-Link DWA-160 WLAN USB 802.11 a/b/g/n dual card (see Figure 2.34a) is installed in the oscilloscope in order to establish a wireless LAN connection with the remote PC as 
shown in Figure 2.27. The frequency of the Wi-Fi connection (determined by the standard a/b/g/n) is chosen in order not to operate in the same band as the frequency band to be sounded.

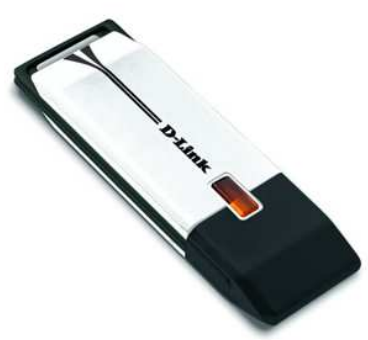

(a)

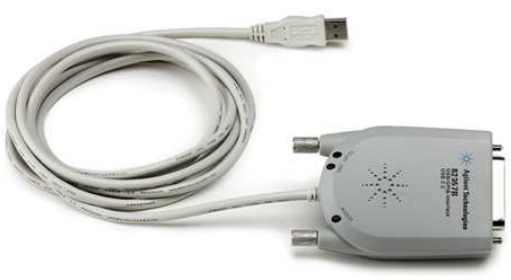

(b)

Figure 2.34. (a) D-Link Wi-Fi card, (b) Agilent USB/GPIB cable.

Connecting the baseband generator with the PC involves the use of the Agilent 82357B USB/GPIB cable shown in Figure 2.34b.

It should be taken into account that the four amplifiers needs $12 \mathrm{~V}$ DC input each one. Thus, two power supplies (one in transmission and one in reception) are also used.

\subsubsection{Software Application}

As mentioned in Section 2.3.2.1. D a software application that is executed in the PC, controls the whole measurement procedure [Alonso Sánchez, 2010], [Solano García, 2011]. This application, shown in Figure 2.35, has been developed in Visual Basic 6 and uses the SCPI (Standard Commands for Programmable Instruments) commands set of all the devices (baseband generator, modulator, and oscilloscope) to configure them correctly depending on the measurement constrains.

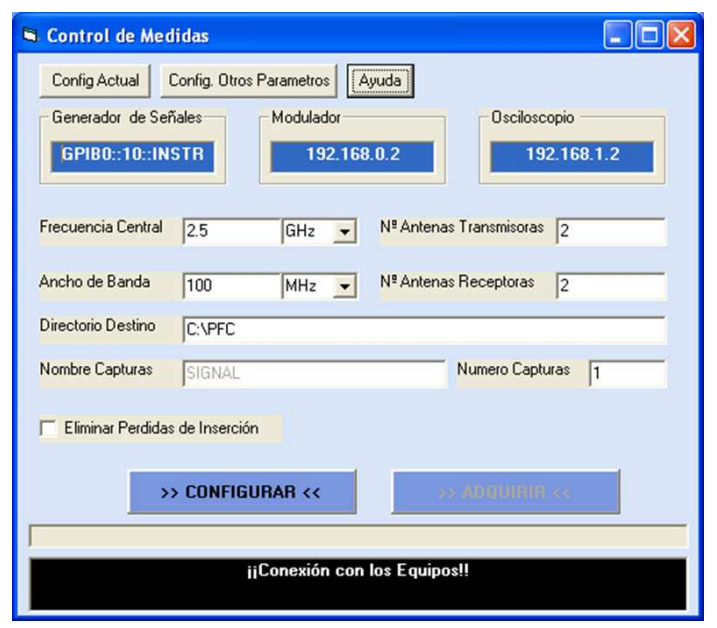

Figure 2.35. Interface of the software application. 
Furthermore, the application executes and control the measurement procedure from the generation of the LS sequences by the baseband generator, to the acquisition and store of the receiving signals on the hard disk of the oscilloscope.

\subsubsection{Synchronization and Delay Compensation}

The synchronization between transmitter and receiver equipment is a crucial issue. In literature one can found, on the one hand, wireless solutions based on rubidium standars [Maharaj et al., 2005], [Kivinen et al., 1999], [Salous et al., 2005] or Global Positioning System (GPS) devices [Chung et al., 2005]. On the other hand, wired approach are also used [Ciccognani et al., 2005], [Keignart et al., 2006] by connecting transmitter and received through a coaxial cable.

In the case of the time-domain channel sounder here presented, the wired solution was selected because of its simplicity and cost. Furthermore, the GPS option was not possible due to the fact that the Agilent baseband generator should be triggered slower than the repetition frequency of the code, $f_{r}$.

The triggering system is implemented by connecting the trigger output of the baseband signal generator with the trigger input of the oscilloscope via a coaxial cable as shown in Figure2.27 Thus, transmitting and receiving sides are correctly synchronized.

Besides, the delay introduced by each element of the channel sounder (cables, filters and amplifiers) from the baseband generator to the scope inputs should be compensated. To accomplish this, we connect the last element (output cable of the transmitting amplifier) with the transmitting side and the first element of the receiving side (input port of the filter). One can observe in Figure 2.36 how the receiving signals are not correctly aligned with the trigger signal (due to de delay introduced by cables, amplifiers and filters). Such delay is measured and introduced as an input parameter of the software application in order to be compensated in the measurements.

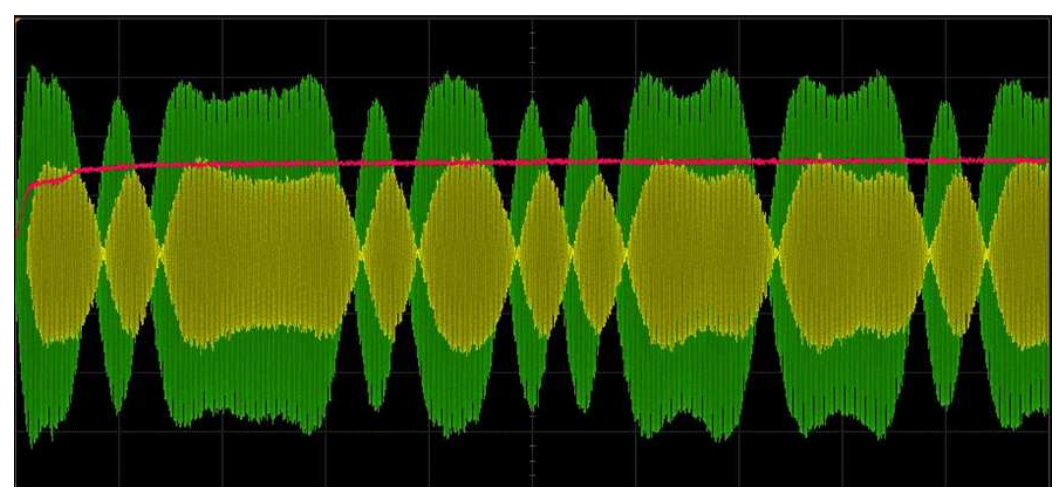

Figure 2.36. Misalignment between trigger and received signals. 


\subsubsection{Dynamic Range}

One of the drawbacks of the time-domain channel sounders is the low dynamic range compared to the frequency-domain channel sounders based on a network analyzer [Rappaport, 1996. Therefore, it is necessary to know the limits of the time-domain implemented, which will depend on the technical characteristics of each device.

To analyze this point, let us assume the most adverse situation, where the signal level is about the noise level. As mentioned before, it should be taken into account that the noise level of the oscilloscope (minimum signal than the scope can acquire) is determined by the scale used as presented in Table 2.5. Thus, the maximum path loss that can be measured will depend on the scale used to acquire the signal.

\begin{tabular}{||ccc||}
\hline \hline Scale (mV/div) & Vmin $_{\left(\mathbf{m V}_{\mathbf{r m s}}\right)}$ & Pmin $\mathbf{( d B m )}$ \\
\hline 5 & 0.322 & -59.83 \\
\hline 10 & 0.358 & -55.91 \\
\hline 20 & 0.498 & -53.05 \\
\hline 50 & 1.150 & -45.78 \\
\hline 100 & 2.220 & -40.06 \\
\hline 200 & 4.280 & -34.36 \\
\hline 500 & 11.500 & -25.78 \\
\hline 1000 & 22.300 & -20.02 \\
\hline \hline
\end{tabular}

Table 2.5. Minimum voltage and power that can be measured by the oscilloscope.

However, after some experiments, it was determined that due to the fact that the vertical scale is 8 bits in its 8 vertical divisions, the minimum signal vertical span should be at least 3 divisions in order to be able to post-process (see Section 2.3.2.5) the signal correctly. This involves that the minimum acceptable power in each scale should be corrected to values shown in Table 2.6 ,

\begin{tabular}{||cc||}
\hline \hline Scale (mV/div) & $\mathbf{P ~ ( d B m ) ~}$ \\
\hline 5 & -23.47 \\
\hline 10 & -17.45 \\
\hline 20 & -11.43 \\
\hline 50 & -3.47 \\
\hline 100 & 2.55 \\
\hline 200 & 8.57 \\
\hline 500 & 16.53 \\
\hline 1000 & 22.55 \\
\hline \hline
\end{tabular}

Table 2.6. Minimum acceptable power in the oscilloscope depending on the vertical scale. 
Assuming that the antennas have a gain of $1 \mathrm{dBi}$ and (as used in measurements in the following sections) and the parameters of each device in Figure 2.23, the gain of the transmitting and receiving side are summarized in Table 2.7 .

\begin{tabular}{||cccc||}
\hline \hline Device TX & Gain (dB) & Device RX & Gain (dB) \\
\hline Coaxial Cable & -1 & Antenna & 1 \\
\hline Amplifier & 30 & Coaxial Cable & -1 \\
\hline Coaxial Cable & -1 & Amplifier & 30 \\
\hline Antenna & 1 & Coaxial Cable & -1 \\
\hline & & Filter & -2 \\
\hline TOTAL TX & 29 & TOTAL RX & 27 \\
\hline \hline
\end{tabular}

Table 2.7. Gain in the transmitting side.

Consequently, considering all the aspects mentioned before, the maximum path loss that can be measured with this new time-domain channel sounder is summarized in Table 2.8. It should be remarked that in this case, the transmitting power of the modulator (see Section 2.3.2.1.B) was considered to be at its maximum $(19 \mathrm{dBm})$, so the amplifiers are working in their linear zone.

\begin{tabular}{||cc||}
\hline \hline Scale (mV/div) & Lmax (dB) \\
\hline 5 & 98.47 \\
\hline 10 & 92.45 \\
\hline 20 & 86.43 \\
\hline 50 & 78.47 \\
\hline 100 & 72.45 \\
\hline 200 & 66.47 \\
\hline 500 & 58.47 \\
\hline 1000 & 52.45 \\
\hline \hline
\end{tabular}

Table 2.8. Gain in the receiving side.

\subsubsection{Post-Processing}

After the measurement routine, the received signals $s_{R X_{1}}(t)$ and $s_{R X_{2}}(t)$ are acquired and stored in the oscilloscope. Then, the aim is to compute each sub-channel impulse response, $h_{i j}(t)$, of the MIMO transfer matrix when the antenna $i$ is receiving and the antenna $j$ is transmitting as:

$$
\boldsymbol{H}(\tau)=\left[\begin{array}{ll}
h_{11}(\tau) & h_{12}(\tau) \\
h_{21}(\tau) & h_{22}(\tau)
\end{array}\right]
$$

As a previous step in the post-processing procedure, the received signal is filtered in order to post-process only the band of interest, $f_{0} \pm f_{\text {chip }}$. 
Then, in order to coherently demodulate each received signal, $s_{R X_{i}}(t)$, we use a carrier at the frequency of the transmitted signal, $f_{0}$, to extract its in-phase, $r_{i}^{I}(t)$, and quadrature-phase, $r_{i}^{Q}(t)$, components [Ciccognani et al., 2005]. Demodulation is completed by using a low-pass Butterworth filter due to its simplicity.

Due to the properties of the LS codes mentioned before, the in-phase and quadrature-phase components of the channel impulse response, $h_{i j}^{I}(\tau)$ and $h_{i j}^{\mathrm{Q}}(\tau)$, can be obtained by correlating $r_{i}^{I}(t)$ and $r_{i}^{Q}(t)$ with $c_{j}(t)$, respectively. Finally, each sub-channel impulse response $h_{i j}(\tau)$ is computed as

$$
\left|h_{i j}(\tau)\right|=\sqrt{\left(h_{i j}^{I}(\tau)\right)^{2}+\left(h_{i j}^{Q}(\tau)\right)^{2}}
$$

and

$$
\phi_{h_{i j}(\tau)}=\arctan \left(\frac{h_{i j}^{Q}(\tau)}{h_{i j}^{I}(\tau)}\right)
$$

Once the whole matrix $\boldsymbol{H}(\tau)$ has been computed, the MIMO channel parameters can be analyzed as usual. Because of the features of LS codes mentioned before, the channel impulse response $h_{i j}(\tau)$ is only computed in the IFW, whose length is

$$
I F W=2 L\left(\frac{f_{s}}{f_{\text {chip }}}\right)-1
$$

samples, where $f_{s}$ is the sampling frequency of the oscilloscope. Hence, the maximum delay that can be measured by the channel sounder is

$$
\tau_{\text {max }}=\tau_{\text {IFW }}=T_{S}\left(L\left(\frac{f_{s}}{f_{\text {chip }}}\right)-1\right)
$$

where $T_{s}$ is the sampling period of the oscilloscope. The delay resolution is equal to the bit rate

$$
\Delta \tau=T_{\text {chip }}=1 / f_{\text {chip }}
$$

\subsubsection{Validation of the Channel Sounder}

As mentioned before, in order to evaluate and validate the performance of the new time-domain MIMO channel sounder, some $2 \times 2 \mathrm{MIMO}$ indoor measurements in a corridor have been performed. The results are compared with those obtained at the same position with our frequency-domain channel sounder based on a multiport network analyzer presented in Section 2.2 and detailed in Appendix C. The measurement environment is a small corridor as depicted in Figure 2.37 The walls are made of plasterboard, the floor is made of reinforced concrete, and so is the ceiling. 


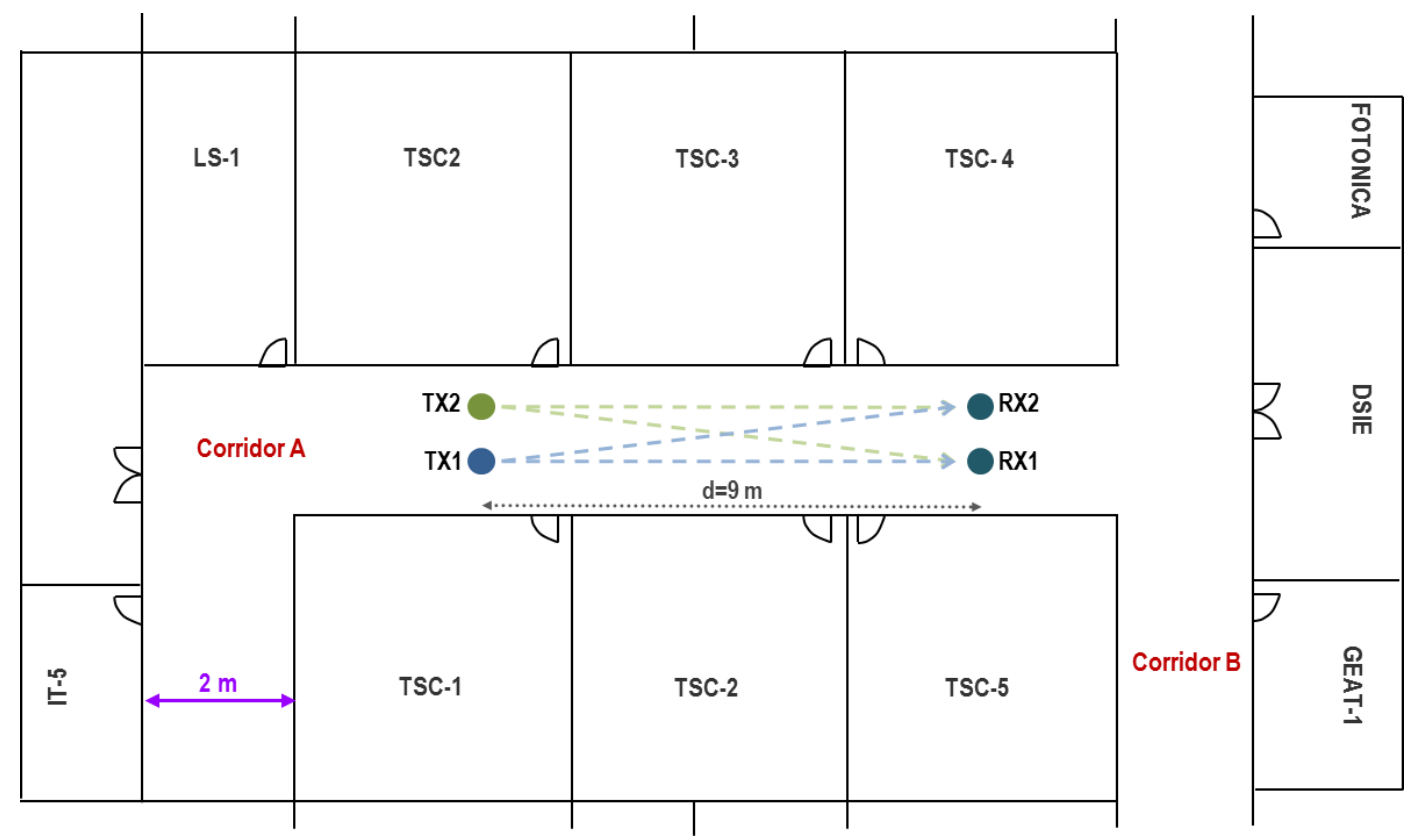

Figure 2.37. Measurement environment.

The frequency band analyzed is $100 \mathrm{MHz}$ around $3.5 \mathrm{GHz}(3.45-3.55 \mathrm{GHz})$ and the separation between elements is $2 \lambda$ at both the transmitting and receiving arrays. The measurement parameters are summarized in Table 2.9 .

\begin{tabular}{||cc||}
\hline \hline Parameter & Value \\
\hline Central Frequency $\left(f_{c}\right)$ & $3.5 \mathrm{GHz}$ \\
\hline Bandwidth $(B W)$ & $100 \mathrm{MHz}$ \\
\hline Wavelength $(\lambda)$ & $8.6 \mathrm{~cm}$ \\
\hline Spacing $\left(\Delta \lambda_{T X}=\Delta \lambda_{R X}\right)$ & $\approx 2 \lambda$ \\
\hline \hline
\end{tabular}

Table 2.9. Measurement Parameters.

In the time-domain channel sounder, the code length was set to $C=510$ bits ( $L=128)$ so the repetition frequency of the base-band generator, $f_{r}$, is $196.08 \mathrm{kHz}$ (chip frequency of $100 \mathrm{MHz}$ ). The speed of the system depends, on the one hand, on the acquisition time (duration of the code), $1 / f_{r}$, which determines also the time during which the channel should be invariant. On the other hand, the speed depends also on the time needed to save the signal in the oscilloscope's hard disk. Thus, the overall time (acquisition + saving) will condition the number of channel impulse responses measured per second (CIRs/s).

Therefore, the configuration of the time-domain and frequency-domain channel sounders are detailed in Table 2.10 and Table 2.11 respectively. 


\begin{tabular}{||cc||}
\hline \hline Parameter & Value \\
\hline Code Length $(C)$ & 510 bits \\
\hline Complementary Pair Length $(L)$ & $128 \mathrm{bits}$ \\
\hline Chip Frequency $\left(f_{r}\right)$ & $196.08 \mathrm{kHz}$ \\
\hline Acquisition Time & $5.1 \mu \mathrm{s}$ \\
\hline Speed & $4 \mathrm{CIRs} / \mathrm{s}$ \\
\hline Vertical Scale & $1 \mathrm{~V} / \mathrm{div}$ \\
\hline Horizontal Scale & $561 \mathrm{~ns} / \mathrm{div}$ \\
\hline Transmitting Power & $-42 \mathrm{dBm}$ \\
\hline$\tau_{\max }$ & $1.28 \mu \mathrm{s}$ \\
\hline$\Delta \tau$ & $10 \mathrm{~ns}$ \\
\hline \hline
\end{tabular}

Table 2.10. Configuration parameters of the time-domain channel sounder.

\begin{tabular}{||cc||}
\hline \hline Parameter & Value \\
\hline Transmitting Power & $0 \mathrm{dBm}$ \\
\hline Number of Points & 1601 \\
\hline$\tau_{\max }$ & $16 \mu \mathrm{s}$ \\
\hline$\Delta \tau$ & $10 \mathrm{~ns}$ \\
\hline \hline
\end{tabular}

Table 2.11. Configuration parameters of the frequency-domain channel sounder.

The normalized power-delay profile (PDP), which represents the received power in the delay domain (see Appendix B, Section B.5), has been computed for each channel impulse response, $h_{i j}$, of the MIMO matrix, $\boldsymbol{H}(\tau)$ as:

$$
\operatorname{PDP}_{i j}(\tau)=\left|h_{i j}(\tau)\right|^{2}
$$

and it is depicted in Figure 2.38

One can observe how both PDPs exhibit the same behavior. Nevertheless, it should be taken into account that the response obtained by the time-domain channel sounder is a snapshot of the channel impulse response, while the response provided by the frequency-domain channel sounder represents the permanent channel impulse response. Consequently, the time-domain PDP displays a more instantaneous behavior than the frequency-domain one.

Furthermore, as a consequence of the autocorrelation properties of the codes used, the timedomain response also shows a characteristic crinkly response observed in Figure 2.38 , 

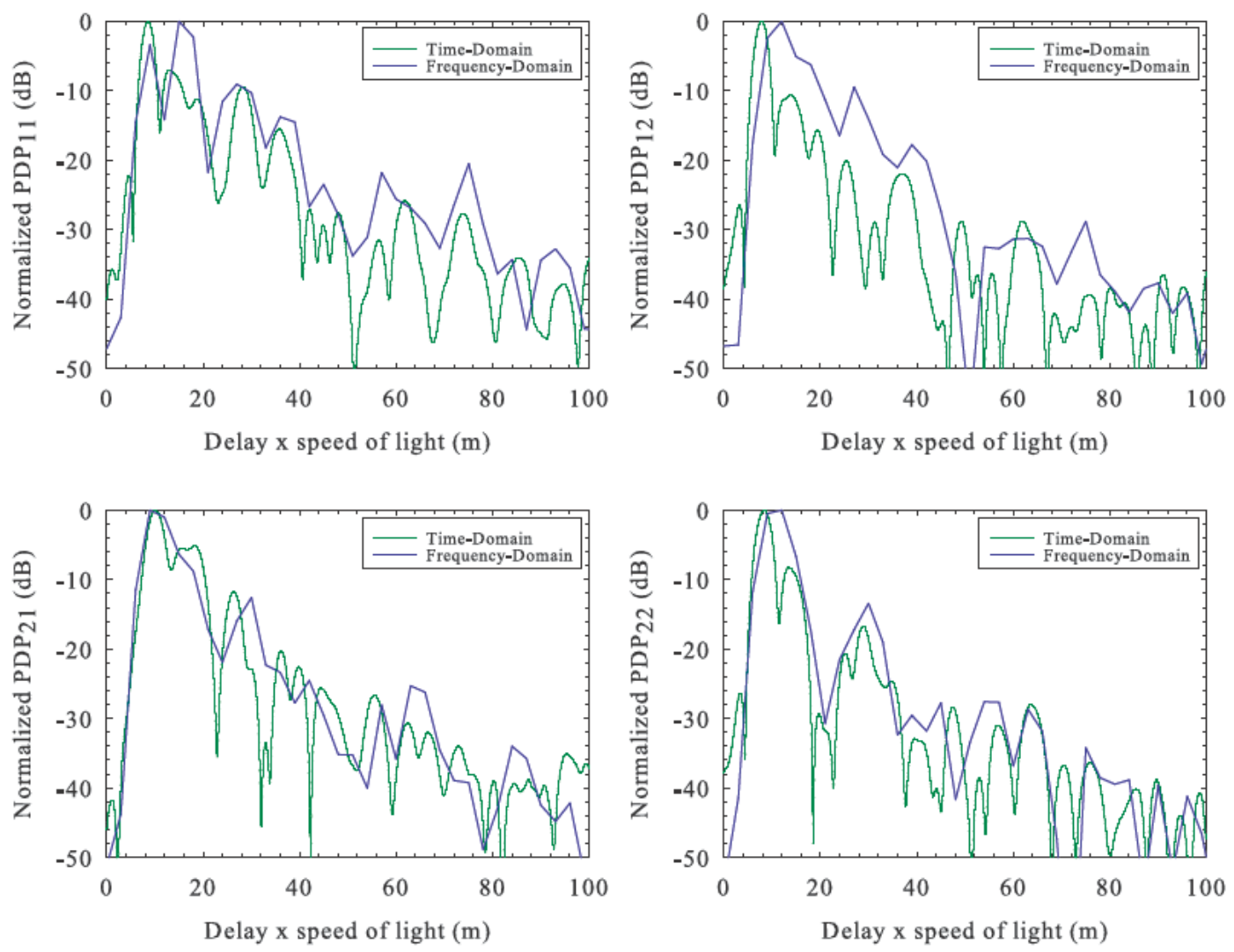

Figure 2.38. Normalized power-delay profile of the MIMO transfer matrix.

To compare the performance of single antenna parameters, the mean RMS delay spread (see Appendix B Section B.5.2) has been computed as:

$$
\sigma_{\tau}=\sqrt{\frac{\sum_{k} P\left(\tau_{k}\right) \tau_{k}^{2}}{\sum_{k} P\left(\tau_{k}\right)}-\left(\frac{\sum_{k} P\left(\tau_{k}\right) \tau_{k}}{\sum_{k} P\left(\tau_{k}\right)}\right)^{2}}
$$

where $P\left(\tau_{k}\right)$ is the received power (in linear units) at $\tau_{k}$. The results are summarized in Table 2.12 for different thresholds. Regarding multiantenna parameters, the complex correlation coefficients of the transmission and reception antennas are computed as explained in Appendix $\mathrm{A}$ Section $\mathrm{A} .3$ and summarized in Table 2.12. In both cases, a quite good agreement is shown for both channel sounders.

\begin{tabular}{||ccccc||}
\hline \hline Channel Sounder & RMS $_{-10 \mathrm{~dB}}$ & RMS $_{-20 \mathrm{~dB}}$ & TX Corr & RX Corr \\
\hline Time-Domain (SCOPE & $8.80 \mathrm{~ns}$ & $16.4 \mathrm{~ns}$ & 0.65 & 0.67 \\
\hline Frequency-Domain (ENA & $10.5 \mathrm{~ns}$ & $21.2 \mathrm{~ns}$ & 0.66 & 0.68 \\
\hline \hline
\end{tabular}

Table 2.12. RMS delay spread and correlation values for both channel sounders. 


\subsection{Conclusions}

This chapter has dealt with the improvement and development of two MIMO channel sounders. On the one hand some improvements were performed to an existing frequency-domain MIMO channel sounder based on a multiport network analyzer in order to obtain new features and accomplish some special kind of channel measurements. On the other hand, a new MIMO timedomain channel sounder has been developed.

Firstly, an existing frequency-domain channel sounder based on a multiport network analyzer was modified in order to accomplish high resolution measurements in the spatial domain. To accomplish this an $\mathrm{XY}$ positioning board and two stepper motors were used. It was shown how the RMS delay spread varied significantly from one position to another within an area of $60 \times 60$ $\mathrm{cm}^{2}$. Furthermore, the complex receiving correlation was calculated in such a small area, showing that when transmitter and receiver are NLoS, the correlation decreases more rapidly than in LoS situations.

Secondly, the same channel sounder was also integrated in a geographical information system (GIS) with the aim of control all the measurement campaigns from a geographic point of view. Thus, measurements are georeferenced as well as the locations of both transmitter and receiver. This application could very useful for analyzing and comparing the radioelectric parameters provided by measurements and those provided by theoretical simulations that can be run into the GIS. Furthermore, the application make the organization and storing of the measurements easier.

Finally, a $2 \times 2$ MIMO time-domain channel sounder based on the use of commercial and multipurpose equipment has been developed. The main advantage of this kind of channel sounders is the low acquisition time compared to classical channel sounders based on a network analyzer. This measurement system is based on the transmission and correlation of LS sequences, which have special autocorrelation and cross-correlation properties. The channel sounder has been made up with multipurpose and commercial equipment such as a base-band generator, an I/Q modulator, and an oscilloscope. The delay introduced by each device was compensated before starting measurements. In order to extract the channel impulse response from the received signals, a postprocessing procedure is followed after finishing measurements. The received signals are demodulated In-Phase and in Quadrature-Phase in order to obtain the complex channel impulse response in the delay domain.

Measurements in a corridor have also been undertaken in order to compare the channel impulse response provided by this new time-domain channel sounder with the one existing in the frequencydomain. It was observed that the channel response measured by both sounders exhibited a similar behavior; also, the RMS delay spread and correlation coefficients computed show similar values. 


\section{Chapter 3}

\section{Polarized MIMO-UWB Channel Modeling}

In this chapter, the polarized Multiple-Input Multiple-Output Ultra-Wideband (MIMO-UWB) channel has been experimentally analyzed by means of an indoor measurement campaign in the $2-5 \mathrm{GHz}$ frequency band [Garrido Cervantes, 2010]. The parameters extracted from this analysis have been compared with those obtained in the same environment for a $200 \mathrm{MHz}$ bandwidth [Molina-Garcia-Pardo et al., 2008c].

Firstly, in Section 3.1 the state of the art about UWB and polarized MIMO-UWB channel modeling is reviewed.

In Section 3.2 the channel sounder, the scenarios where measurements were carried out and the polarizations of the arrays are described.

In Section 3.3 the measured path loss is analyzed and path loss models for co-polar polarizations are proposed for each scenario. This analysis is performed as a function of the distance between transmitter and receiver and as a function of frequency. The influence of polarization is studied by analizing the XPD (Cross Polar Discrimination Factor) in both space and frequency domain.

Next, Section 3.4 deals with the analysis of the delay dispersion for all the scenarios and all 
polarizations. As in the case of the path loss, the effect of both distance and frequency on the RMS (Root Mean Square) delay spread is considered.

Regarding multiantenna parameters, in Section 3.5 the MIMO system capacity is evaluated for all the polarizations. The capacity is computed under two different assumptions: in case of constant transmitted power or constant received power.

Finally, The main conclusions deduced from the work presented in this chapter are outlined in Section 3.6 ,

\subsection{State of the Art of UWB Channel Modeling}

Ultra-Wideband (UWB) communications have lately gained much interest from the research community [Win and Scholtz, 1998], [Molisch, 2005], [Qiu et al., 2005], [Win et al., 2009]. UWB signals are defined as those whose bandwidth is larger than $500 \mathrm{MHz}$ (UWB with a large absolute bandwidth) and/or larger than $20 \%$ of the central frequency (UWB with a large relative bandwidth) [Molisch, 2009]. The main features offered by UWB are extremely high data rates, precise ranging and geolocation and covert high-data-rate communications. The development of applications that requires such capabilities are Personal Area Networks (PAN), Wireless Sensor Networks (WSN), emergency communications, geolocation, Body Area Networks (BAN), and so on.

However, due to its coexistence with other technologies, the regulatory organisms have strongly restricted the transmitted power of UWB systems, due to the interference with existent communication systems, as it is shown in Figure 3.1. The Federal Communications Commission (FCC) establishes for indoor environments, a maximum Equivalent Isotropic Radiated Power (EIRP) of $-41.3 \mathrm{dBm} / \mathrm{MHz}$ between 3.1 and $10.6 \mathrm{GHz}$ [FCC, 2002]. In Europe, the European Conference of Postal and Telecommunication Administrations (CEPT) imposes even more stringent limits for UWB emissions [ECO, 2009]. These restrictions make necessary a thorough study of the propagation channel in order to determine the limits of both the characteristics of transmitters and receivers and its algorithms [Benedetto et al., 2006].

A fundamental mechanism in wireless propagation is multipath propagation: the fact that the signal can get from the transmitter $(\overline{T X})$ to the receiver $(\underline{\mathrm{RX}})$ via different paths and interactions (reflection, diffraction, diffusion...). Depending on the path that a multipath contribution (MPC) takes, it has a certain delay, attenuation, and direction of arrival. Therefore the signal arriving at the receiver is the sum of scaled and delayed replicas of the transmit signal and the channel impulse response of the channel is [Molisch, 2009]:

$$
h(t, \tau)=\sum_{i=1}^{N} a_{i}(t) \delta\left(\tau-\tau_{i}\right)
$$




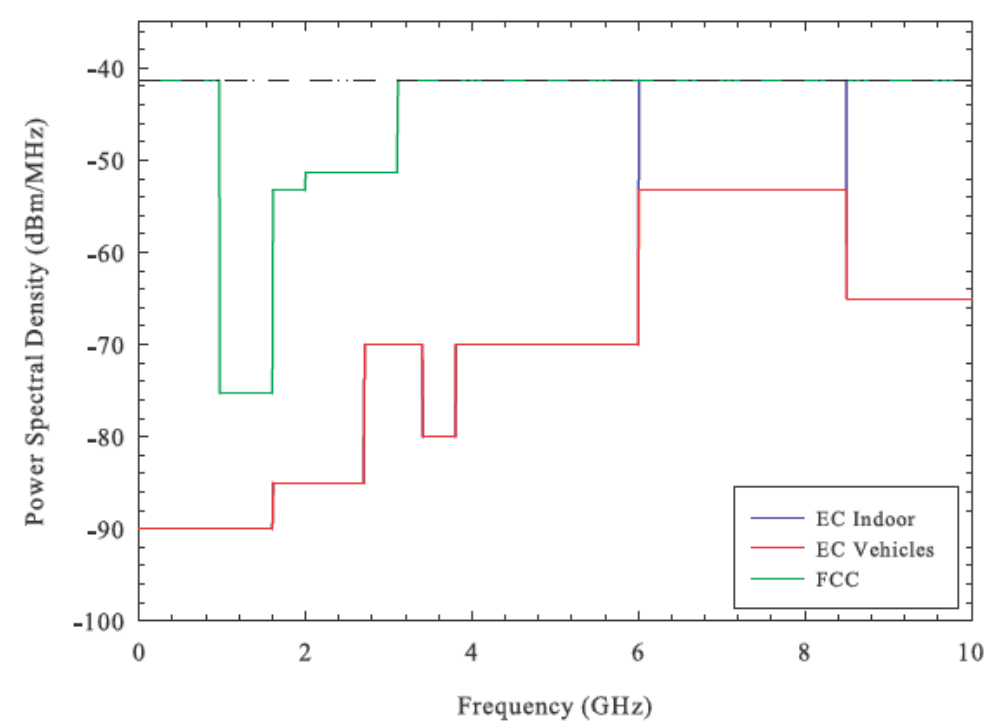

Figure 3.1. Emissions regulation in Europe (EC) and the United States (FCC)..

where $a_{i}$ and $\tau_{i}$ are the gain and delay of the ith MPC. However, due to the large bandwidth occupied by UWB signals (larger than common wideband signals), some propagation phenomena do not behave in the same way at all frequencies. Consequently, the transmitted signal suffers from distortion, and then the channel impulse response is described as Molisch, 2005, [Molisch et al., 2006], [Molisch, 2009]:

$$
h(t, \tau)=\sum_{i=1}^{N} a_{i}(t) \chi_{i}(t, \tau) \bigotimes \delta\left(\tau-\tau_{i}\right)
$$

This distortion makes that some parameters such as the path loss not only depends on distance but also on frequency. Therefore, both distance and frequency dependence should be considered when estimating the received power.

Despite the importance of frequency in UWB channels, there is not much information in the scientific literature about the effect of frequency on the essential parameters of the UWB propagation channel. In [Choi et al., 2006a] and [Choi et al., 2009], the decay factor of the path loss is studied in the frequency domain, while in [Chong et al., 2005] the effect of distance and frequency on the path loss is examined.

\subsubsection{Polarized MIMO-UWB Channel Modeling}

The polarization of the radio waves plays also an important role in propagation. If the receiver's polarization does not perfectly match that of the transmitter, the receiving antenna will not be able to capture all the received power. Therefore, knowledge of the behavior of the polar 
radio channel has been frequently addressed in the literature. The so called tap XPD and energy XPD and their relationship with parameters such as the RMS delay spread is widely studied in Malik, 2008a from measurements using elliptical polarization. Indoor channel measurements with dual-polarized or hybrid array configurations have been reported in [Kyritsi et al., 2002], [Zhao et al., 2003], [Wallace et al., 2003].

Multiple-input multiple-output (MIMO) (see Appendix A) systems were considered as one of the best techniques to optimize the use of the transmission spectrum and power [Winters, 1987], [Foschini and Gans, 1998]. This technique benefits from the use of multiple antennas at both sides of the radio interface, so that by using a proper space-time code, either the diversity and/or the throughput can be substantially improved [Gesbert et al., 2003]. The use of MIMO in UWB systems has been addressed as one possible solution to considerably improve the UWB link robustness or its range [Sibille, 2005] as well as the error-rate [Wang et al., 2005], [Yang and Giannakis, 2004], [Liu et al., 2005]. In contrast with narrowband channels, there are few studies of the use of the MIMO technique in UWB channels. In [Malik and Edwards, 2007], [Zheng and Kaiser, 2004], [Zheng and Kaiser, 2008] some results on the channel capacity of UWB-MIMO systems are presented. Furthermore, in [Malik and Edwards, 2007] it was found that polarization diversity is sometimes more effective than temporal multipath diversity, so the required number of rake fingers can be reduced. In [Malik, 2008b] the author demonstrates that horizontally polarized links exhibit less spatial correlation than vertically polarized links. However, although polarization has been widely analyzed for conventional MIMO systems [Erceg et al., 2004], [Oestges et al., 2004], [Eiceg et al., 2006], [Shafi et al., 2006], [Bhagavatula et al., 2010], [Quitin et al., 2009b], [Quitin et al., 2010] there is not too much information in scientific literature about the effect of polarization in MIMO-UWB systems.

\subsection{Description of the Measurement Campaign}

The measurement campaign has been carried out in the underground level of the Universidad Politécnica de Cartagena. It consists mainly of a 50 meter long corridor, three perpendicular corridors, and some labs. A top view of the selected indoor environment is depicted in Figure 3.2 The walls of this building are made of plasterboard, and the floor and ceiling are made of reinforced concrete.

The MIMO-channel sounder used to perform the measurements (see Figure 3.3) is based on a multiport vector network analyzer (MVNA), composed by an Agilent ENA E5071B and Multiport Test Set E5091A, and a solid state fast switch, (Agilent 87406B, controlled by a switch driver Agilent 11713A) [Molina-Garcia-Pardo et al., 2008c]. A more detailed explanation about this channel sounder and the equipment is presented in Appendix $\mathrm{C}$

The receiving antennas are directly connected to the ports of the MVNA. One port of the MVNA 


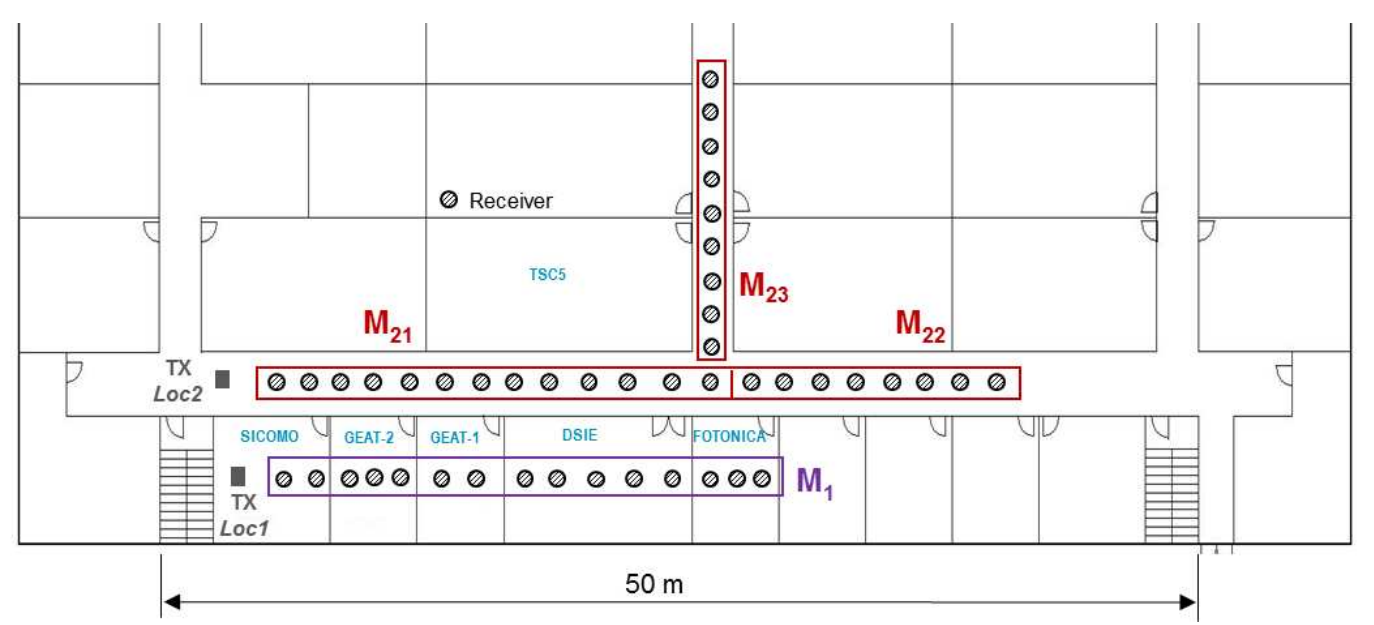

Figure 3.2. Floor plan of the measurement scenario and the position of the transmitting and receiving antennas.

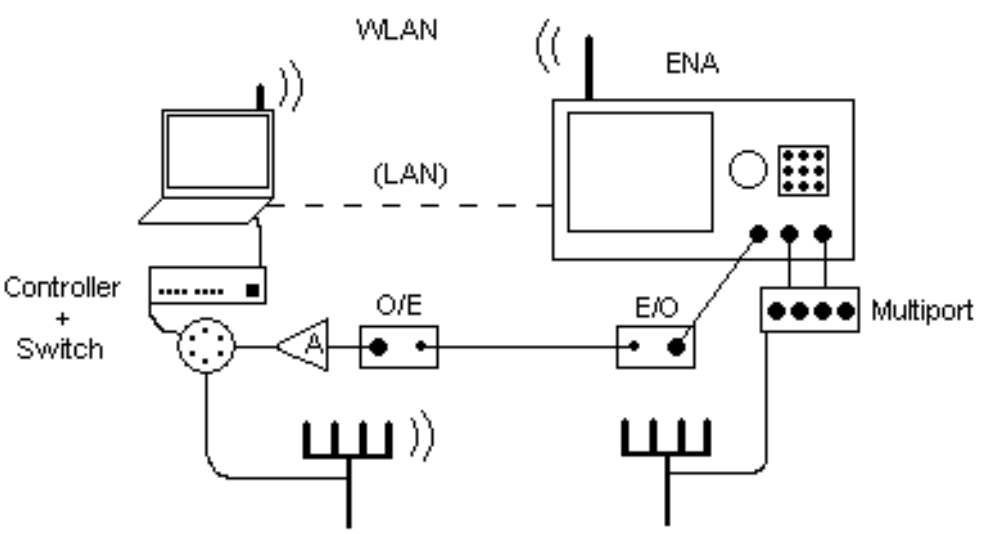

Figure 3.3. Block diagram of the MIMO channel sounder.

is configured as a transmitter and connected to an optical link (RF/OF and OF/RF), which carries the signal to the fast switch. Such optical link is composed of an optic fiber of 50 meters long, a Ortel 3540A transmitter (RF/OF) and a Ortel 4510B optical receiver (OF/RF). Finally, the transmitting antennas are connected to the fast switch, so that the signal from the optical link is transmitted sequentially to each element of the transmitter array. The measurement process is controlled by a developed program executed on a laptop, which is connected to the MVNA by a LAN (Local Area Network) and to the fast switch by a GPIB (General-Purpose Interface Bus) port. The whole measurement system (MVNA, optical link, fast switch, coaxial cables and connectors) is calibrated before sounding the channel in order to remove the frequency response of all elements from the measurements. This calibration procedure is repeated when starting measurements for each zone in Figure 3.2 .

For the measurements, eight Electro-Metrics omnidirectional mast mounted antennas (EM-6116) with $1 \mathrm{dBi}$ gain which work in the 2-10 GHz frequency band with a XPD of $12 \mathrm{~dB}$ have been used. 
The power transmitted by each antenna was $-13 \mathrm{dBm}$ and the dynamic range after calibration was $95 \mathrm{~dB}$ which was enough to assure a high signal to noise ratio (SNR) for most of the measurements. Therefore, the noise floor measured by the MVNA was $-108 \mathrm{dBm}$.

However, due to the limitations of the optical transmitter and receiver, which work up to $5 \mathrm{GHz}$, the maximum frequency that can be measured is $5 \mathrm{GHz}$. Thus, the channel has been sounded from $f_{\min }=2$ to $f_{\max }=5 \mathrm{GHz}$ by measuring 801 frequency points over the $B W=3 \mathrm{GHz}$ bandwidth. Therefore, the resulting frequency resolution is $\Delta f=3.75 \mathrm{MHz}$.

The channel coherence bandwidth is defined as the range of frequencies over which the channel can be considered to have components with approximately equal gain and linear phase [Rappaport, 1996]. When the correlation function is above 0.9 , the coherence bandwidth was found to be around $W B_{c}=25 \mathrm{MHz}$. This value is much higher than the frequency resolution $\Delta f$, validating thereby, the correct frequency sampling of the measured channel transfer functions within bins of width $\Delta f$. Furthermore, it is in the order of other values extracted in scientific literature for UWB measurements campaigns [Malik and Edwards, 2007].

Two positions of the transmitter have been chosen (Figure 3.2):

- TX-Location1 (TX-Loc1) in the middle of one of the labs (SiCoMo), at a height of 1.5 meters.

- TX-Location2 (TX-Loc2) in the middle of the corridor at a height of 1.5 meters.

The receivers (circles with hatching in Figure 3.3) have been classified into four groups:

- $\mathrm{M}_{1}$ along five small consecutive labs (15 positions).

- $\mathrm{M}_{21}$ along the corridor (first 13 positions).

- $\mathrm{M}_{22}$ along the corridor, after the $\mathrm{T}$ junction (last 8 positions in the corridor).

- $\mathrm{M}_{23}$ along the central perpendicular corridor, after the $\mathrm{T}$ junction (9 positions).

For each position (transmitter-receiver pair), four combinations of the arrays have been measured. Defining $\mathrm{V}$ as the vertical polarization and $\mathrm{H}$ as the horizontal polarization for the elements of the array, the four combinations mentioned are VV, HH, HV and VH (see Figure 3.4), in which the first letter refers to the transmitter and the second to the receiver.

The large size of the antennas meant one was unable to establish a separation between elements of the array of less than $4 \mathrm{~cm}$. Therefore, the antenna spacing had to be set to $2 \lambda_{\min }=6 \mathrm{~cm}$ (with $\lambda_{\min }$ referred to $f_{\max }$ ). 


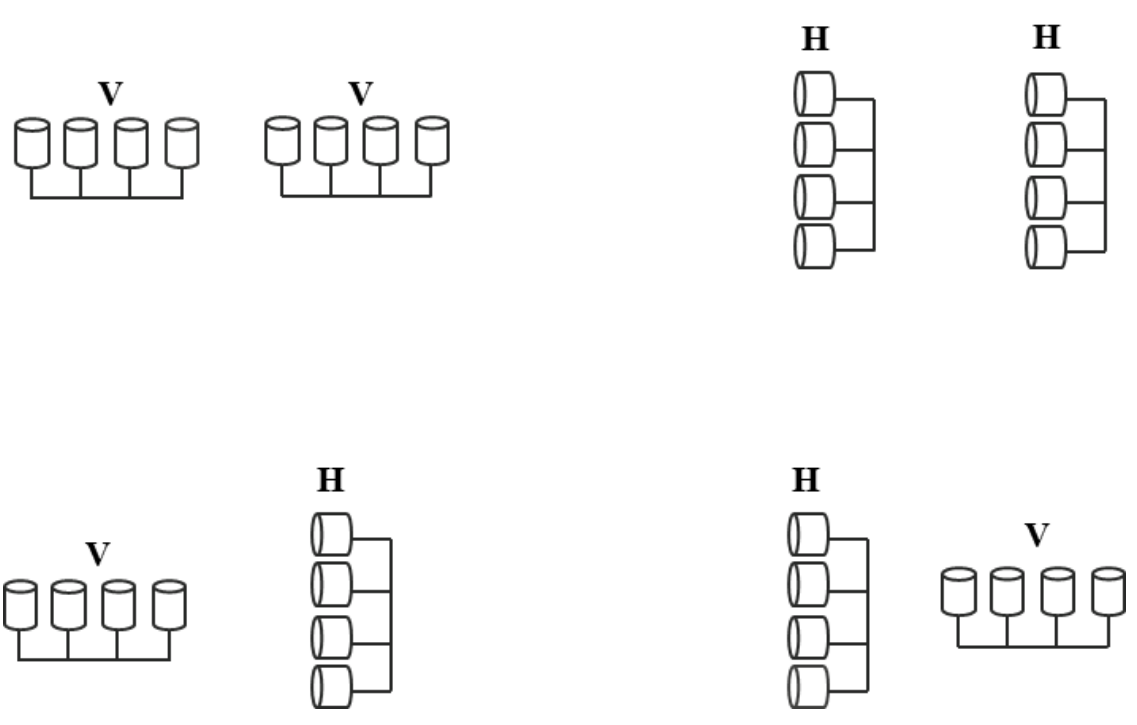

Figure 3.4. Different polarizations for the arrays.

Summarizing, corresponding to each position of the receiver in the route and each polarization, the MIMO frequency domain transfer function is defined as $\mathbf{G}(n, m, f, t)$, where $n$ denotes the receiving element of the array, $m$ the transmitting element of the array, $f$ the frequency point within the measured frequency band, and $t$ the snapshot. Moreover, $n=4$ receiving elements, $m=4$ transmitting elements, and $f=801$ frequency points have been used. The stationarity of the channel is assured by taking $t=5$ snapshots of each measurement, which also improves the Signal-to-Noise Ratio (SNR) of measurements. Thus, for each position and polarization $G$ is a matrix of dimensions $4 \times 4 \times 801 \times 5$.

\subsection{Path Loss Models}

In this section, the path loss is presented, since it is important in any wireless system regardless of the number of antennas. The measured path loss is defined as the ratio of the transmitted power and a local average of the received power (for more information see Appendix B). It can be obtained from the power delay profile (PDP), which represents the received power in the delay domain, which can be computed by averaging spatially the square of the absolute value of the channel impulse response, over a local area [Rappaport, 1996]. In other words, the $P D P(\tau)$ is computed as:

$$
\operatorname{PDP}(\tau)=E_{n, m}\left\{|h(n, m, \tau)|^{2}\right\}
$$

where $E_{n, m}$ is the expectation operator over all receiving antenna elements and transmitting antenna elements, and $h(n, m, \tau)$ is the channel impulse response obtained from the Inverse Fast Fourier Transform (IFFT) of the 801-point frequency response, $\mathbf{G}(n, m, f, t)$. The average of the $t=5$ snapshots is performed in frequency before computing the IFFT in order to improve the SNR. 
Thus, the mean path loss in logarithmic units is calculated from the PDP as [Molina-Garcia-Pardo et al., 2004]:

$$
L=-10 \log _{10} \sum_{i=0}^{N_{f}} \operatorname{PDP}\left(\tau_{i}\right)
$$

where $N_{f}$ is the number of points of the PDP.

In the technical literature many indoor propagation models can be found for predicting the path loss, but as mentioned before, not much information dealing with the influence of polarization can be found. In [Molina-Garcia-Pardo et al., 2008c] the path loss is computed in the frequency domain for a $200 \mathrm{MHz}$ bandwidth in the same environment and for all the polarizations. The objective in this case is not only to analyze and compare the path loss in the same environment for a wider bandwidth, but also to study the Cross-Polar Discrimination factor (XPD) in this environment. To achieve this goal, different antennas have been used in order to measure the UWB channel in the $2-5 \mathrm{GHz}$ frequency band.

\subsubsection{Propagation along the Corridor}

In this section, detailed analysis will be provided for when the receiver is moving away from the transmitter TX-Loc2 over 40 meters in Line-of-Sight (LoS) (21 positions, $\mathrm{M}_{21}+\mathrm{M}_{22}$ ). The measurements in the case the receiver turns left at the $T$ junction $\left(\mathrm{M}_{23}\right)$ and when the receiver is in a Non Line-of-Sight (NLoS) situation are also evaluated.

Firstly, VV and $\mathrm{HH}$ polarizations are both studied for the $3 \mathrm{GHz}$ frequency band as a function of distance. The effect of frequency is studied by analyzing the measurements in $500 \mathrm{MHz}$ slidingwindow frequency intervals, in order to obtain a continuous response in frequency. Finally, as performed with the co-polar polarizations, $\mathrm{VH}$ and $\mathrm{HV}$ configurations will also be studied by analyzing the XPD in the space and frequency domain.

\subsubsection{Analysis of Co-polar Polarizations}

\section{A. Path Loss Modeling in $3 \mathrm{GHz}$ Bandwidth}

First of all, the results from when the receiver is moving away from the TX-Loc2 transmitter along 40 meters in LoS (21 positions, $\mathrm{M}_{21}+\mathrm{M}_{22}$ ) are presented in Figure 3.5. The results in the case where the receiver turns left at the T junction $\left(\mathrm{M}_{23}\right)$ are also presented in Figure 3.5. As shown in Figure 3.2 . the corner of the $\mathrm{T}$ junction is located at $d_{1}=26$ meters away from the transmitter.

For the group of measurements $\mathrm{M}_{21}+\mathrm{M}_{22}$, one can see how the path loss increases linearly with 


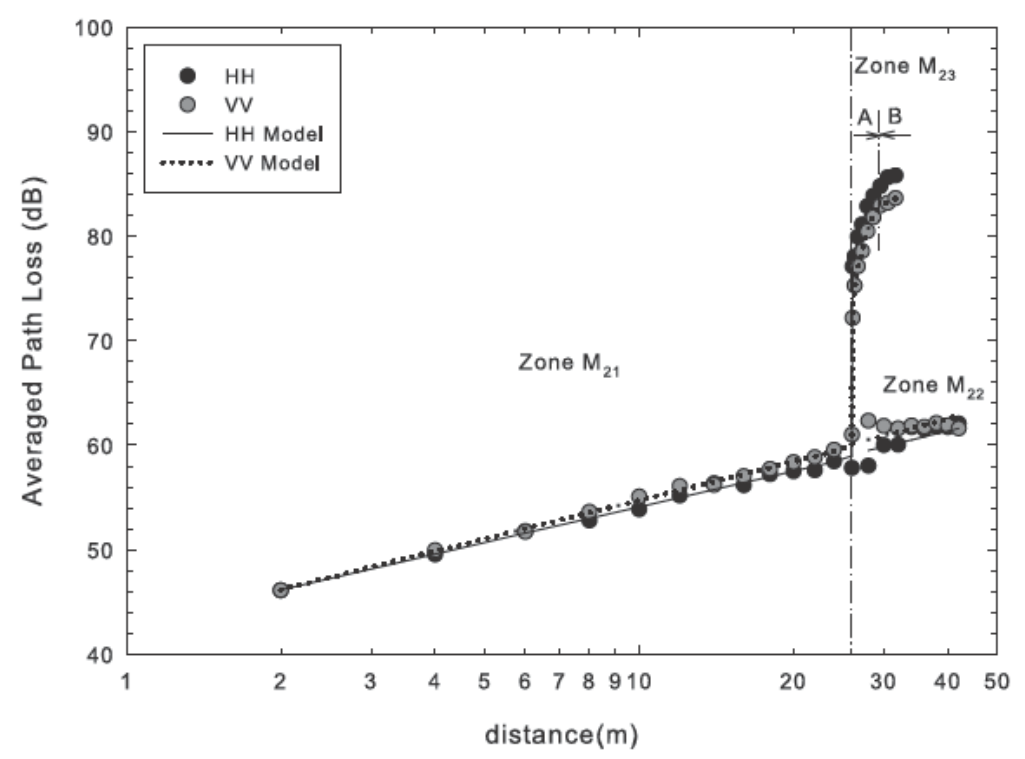

Figure 3.5. Mean path loss for TX-Loc2 and $\mathrm{M}_{21}+\mathrm{M}_{22}$, and $\mathrm{M}_{23}$ and the adjusted models.

distance expressed in logarithmic units, so a one-slope model [Rappaport, 1996] is proposed as:

$$
L(d)=L_{0}+10 n_{1} \log _{10}(d)+X_{\sigma_{1}}
$$

where $L_{0}$ is the path loss at a reference distance $(1 \mathrm{~m}), n_{1}$ is the decay factor, $d$ is the distance between transmitter and receiver, and $X_{\sigma_{1}}$ is the shadowing, which is classically defined as a zero-mean lognormal random variable with standard deviation $\sigma_{1}$.

The statistical distribution of the shadowing was confirmed by conducting a KolmogorovSmirnov test for all polarizations (VV, VH, HH and HV). Results show that the shadowing in logarithmic units follows a normal distribution, confirming thus, the log-normality of this parameter [Ghassemzadeh et al., 2004]. This test was repeated for the measurements in zones $\mathrm{M}_{23}$ and $\mathrm{M}_{1}$, where the same outcomes were found. Therefore, since the shadowing (and therefore the path loss) for $\mathrm{VV}, \mathrm{VH}, \mathrm{HH}$ and $\mathrm{HV}$ is described by the same statistical distribution, the behavior of cross-polar polarizations will be analyzed by means of the computation of its XPD, which will be described by its mean and its standard deviation in the following sections.

In the case of the group of measurements $\mathrm{M}_{23}$, although the path loss clearly increases with distance, two zones can be distinguished: the first one is a transition zone between LoS and NLoS (hereafter Zone $\mathrm{M}_{23_{\mathrm{A}}}$, with the first 6 positions of $\mathrm{M}_{23}$ from distance $d_{1}$ to distance $d_{2}=38 \mathrm{~m}$ ); and the second in NLoS (hereafter Zone $\mathrm{M}_{23-\mathrm{B}}$, with the last 3 positions of $\mathrm{M}_{23}$ from distance $d_{2}$ up to a distance of $44 \mathrm{~m}$ ). For this environment, a two-slope path loss model that defines path loss for a 
group of distances is proposed:

$$
L(d)=\left\{\begin{array}{cc}
L_{0}+10 n_{2} \log _{10}(d)+X_{\sigma_{2}} & d_{1}<d<d_{2} \\
L_{0}+10 n_{3} \log _{10}(d)+X_{\sigma_{3}} & d_{2}<d
\end{array}\right.
$$

The parameters obtained for the path loss models are presented in Table 3.1

\begin{tabular}{||c|ccc|ccc|ccc||}
\hline \hline & \multicolumn{2}{|c|}{ Zone $\mathbf{M}_{21}-\mathbf{M}_{\mathbf{2 2}}$} & \multicolumn{3}{c|}{ Zone $\mathbf{M}_{23_{\mathrm{A}}}$} & \multicolumn{3}{c||}{ Zone $\mathbf{M}_{2 \mathbf{3}_{\mathrm{B}}}$} \\
& $L_{\mathbf{0}}(\mathrm{dB})$ & $n_{\mathbf{1}}$ & $\sigma_{\mathbf{1}}$ & $\boldsymbol{L}_{\mathbf{0}}(\mathrm{dB})$ & $\boldsymbol{n}_{\mathbf{2}}$ & $\sigma_{\mathbf{2}}$ & $L_{0}(\mathrm{~dB})$ & $\boldsymbol{n}_{\mathbf{3}}$ & $\sigma_{3}$ \\
\hline $\mathrm{VV}$ & 41.6 & 1.2 & 1.1 & -233.2 & 21.6 & 1.1 & 47.2 & 2.3 & 0.5 \\
\hline $\mathrm{HH}$ & 41.3 & 1.2 & 1.0 & -151.7 & 16.1 & 0.8 & 42.9 & 2.8 & 0.6 \\
\hline \hline
\end{tabular}

Table 3.1. Whole bandwidth model parameters for the group of measurements $M_{21}+M_{22}$ and $\mathrm{M}_{23}$.

The decay factor indicates the rate at which the path loss increases with distance. On the one hand, the low decay factor obtained here for zones $\mathrm{M}_{21}+\mathrm{M}_{22}$ are approximately of the order of the values proposed by Choi et al. in [Choi et al., 2009] in a similar corridor environment (called Environment 4, in LoS situation). This dramatic reduction of the decay factor is due to the guiding effect present in this environment.

On the other hand, the high decay factor in zone $\mathrm{M}_{23_{\mathrm{A}}}$ is due to the predominance of wallreflected contributions over diffracted contributions. The higher value of $n_{2}$ for VV in this zone could be caused by the existence of some furniture in this zone.

The low values for $\sigma_{1}, \sigma_{2}$, and $\sigma_{3}$ are mainly due to the low number of values for computation $(4 \times 4 \times$ number of measured positions over the distance).

\section{B. Path Loss Modeling in $500 \mathrm{MHz}$ Sliding-Window Intervals}

Next, the influence of frequency on the path loss is studied. To accomplish this, the analysis performed in the previous section is repeated by varying the central frequency of a $500 \mathrm{MHz}$ measurements sliding-window interval [Choi et al., 2009]. In this case, the path loss described by (3.4) is computed from the PDP by computing the N-points IFFT, where $\mathrm{N}$ is now:

$$
N=\frac{500 M H z}{\Delta f}=\frac{500}{3.75} \simeq 133
$$

First of all, the overall effect of the frequency in the path loss for the group of measurements $\mathrm{M}_{21}+\mathrm{M}_{22}$ is considered. To accomplish this, the mean path loss as a function of frequency has been computed by averaging over all the positions along the corridor (see Figure 3.2). The results are depicted in Figure 3.6 


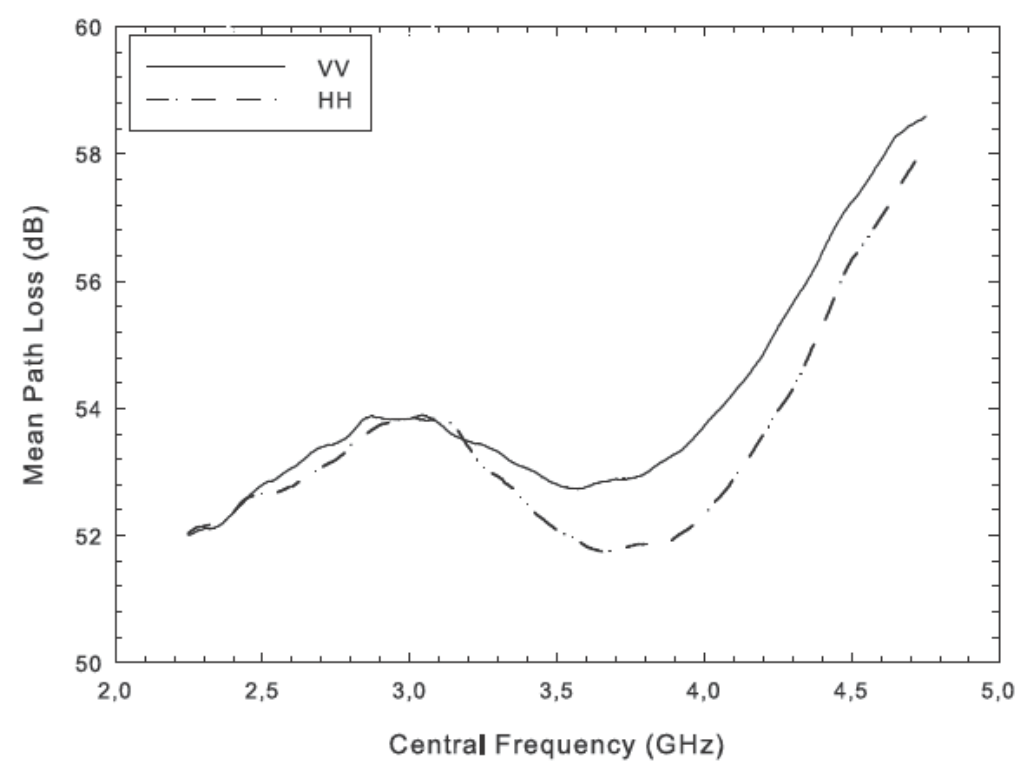

Figure 3.6. Averaged path loss for TX-Loc2 and $\mathrm{M}_{21}+\mathrm{M}_{22}$, as a function of the central frequency of the $500 \mathrm{MHz}$ frequency interval.

The minimum in the path loss between 3.5 and $4.0 \mathrm{GHz}$ observed in Figure 3.6 is due to the behavior of the antennas in this zone owing to the fact that measurements include the effect of the antennas.

The aim of this analysis is also to evaluate the effect of frequency on the parameters of the path loss model proposed in the previous section. To accomplish this, the models proposed in (3.5) and (3.6) have been applied to the measured path loss in each frequency interval. The decay factor remains constant with frequency as observed in Table 3.2 while the parameter $L_{0}$ increases with frequency as summarized in Table 3.3

\begin{tabular}{||c|c|c|c|c|c||}
\hline \hline & $2.5 \mathrm{GHz}$ & $3.0 \mathrm{GHz}$ & $3.5 \mathrm{GHz}$ & $\mathbf{4 . 0} \mathrm{GHz}$ & $\mathbf{4 . 5} \mathrm{GHz}$ \\
\hline VV & 0.9 & 1.3 & 1.3 & 1.3 & 1.3 \\
\hline HH & 1.1 & 1.2 & 1.2 & 1.2 & 1.2 \\
\hline \hline
\end{tabular}

Table 3.2. Decay factor as a function of the central frequency in $\mathrm{M}_{21}+\mathrm{M}_{22}$ zones.

\begin{tabular}{||c|c|c|c|c|c||}
\hline \hline & $2.5 \mathrm{GHz}$ & $\mathbf{3 . 0} \mathrm{GHz}$ & $\mathbf{3 . 5} \mathrm{GHz}$ & $\mathbf{4 . 0} \mathrm{GHz}$ & $\mathbf{4 . 5} \mathrm{GHz}$ \\
\hline VV & 41.8 & 40.3 & 40.1 & 40.1 & 42.3 \\
\hline HH & 40.5 & 40.6 & 40.4 & 39.9 & 42.1 \\
\hline \hline
\end{tabular}

Table 3.3. $L_{0}$ in decibels as a function of the central frequency in $\mathrm{M}_{21}+\mathrm{M}_{22}$ zones. 
In [Choi et al., 2009], the path loss is also analyzed in the frequency domain. However, in this case, the decay factor tends to increase with frequency because of $L_{0}$ does not vary with frequency as in our case.

The same analysis was performed in zone $\mathrm{M}_{23}$ where similar conclusions were extracted.

\subsubsection{Analysis of Cross-polar Polarizations}

Once the co-polar configuration has been analyzed both in the space and frequency domain, the performance of path loss for cross-polar polarization can be studied by means of the analysis of the Cross-Polar Discrimination factor or XPD. In [Malik, 2008a] the author defines the XPD of the UWB channel energy as the ratio between the channel energy of co-polar and cross-polar configurations. Thus, for the configurations presented in Figure 3.4 the XPD can be computed as:

$$
\begin{aligned}
& X P D_{V}(d)=L_{V H}(d)-L_{V V}(d) \\
& X P D_{H}(d)=L_{H V}(d)-L_{H H}(d)
\end{aligned}
$$

In the following sections, the XPD is analyzed in both the space and frequency domain.

\section{A. Analysis of the XPD in a $3 \mathrm{GHz}$ Bandwidth}

First, both the $X P D_{V}$ and $X P D_{H}$ have been computed over the whole bandwidth for each pair of transmitter-receiver elements $(4 \times 4$ in total) for all the positions of the group of measurements $\mathrm{M}_{21}+\mathrm{M}_{22}$. The results are presented in Figure 3.7. In both cases, the XPD can be considered to be nearly constant with distance. The decrease with distance of the XPD provided by the regression line is $1.8 \mathrm{~dB} / 100 \mathrm{~m}$ for $X P D_{V}$ and $0.9 \mathrm{~dB} / 100 \mathrm{~m}$ for $X P D_{H}$. Therefore, the depolarization of the radio waves with distance is more pronounced for the $\mathrm{VV}$ configuration but for very long distances.

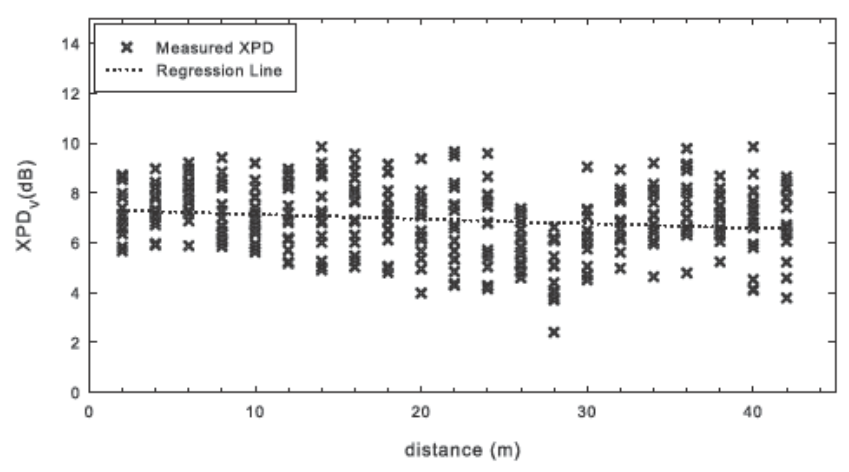

(a)

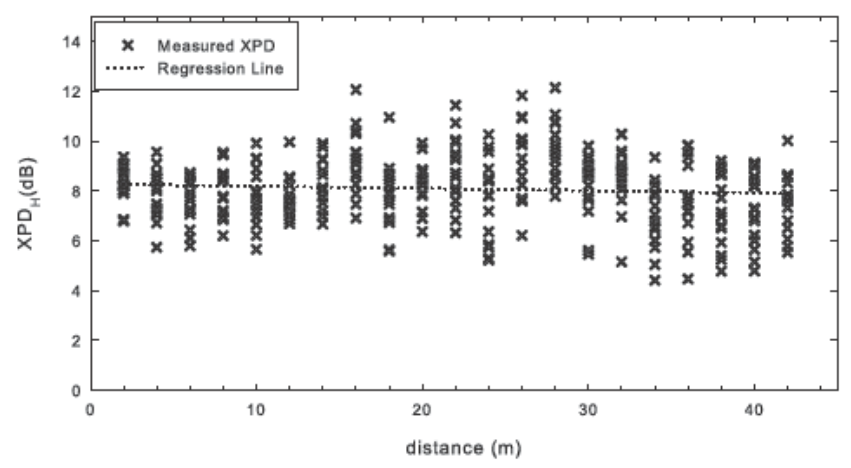

(b)

Figure 3.7. $X P D$ as function of distance for each TX-RX pair: (a) $X P D_{V}$ and (b) $X P D_{H}$. 
The averaged $X P D$ is found to be around $7 \mathrm{~dB}$ for $X P D_{V}$ and around $8 \mathrm{~dB}$ for $X P D_{H}$ with a standard deviation about of $1.4 \mathrm{~dB}$, as summarized in Table 3.4. Similar values of the standard deviation were obtained in [Malik, 2008a], where values were around $1 \mathrm{~dB}$ for all polarizations and LoS situation.

\begin{tabular}{||l|cc|cc||}
\hline \hline & \multicolumn{2}{|c|}{ Zone $\mathbf{M}_{21}-\mathbf{M}_{22}$} & \multicolumn{2}{c||}{ Zone $\mathbf{M}_{23_{\mathrm{A}}}$} \\
& Mean (dB) & Std (dB) & Mean (dB) & Std (dB) \\
\hline$X P D_{V}$ & 7.1 & 1.38 & 7.0 & 1.1 \\
\hline$X P D_{H}$ & 8.1 & 1.41 & 5.6 & 1.1 \\
\hline \hline
\end{tabular}

Table 3.4. Mean and standard deviation of the XPD in zones $\mathrm{M}_{21}+\mathrm{M}_{22}$ and $\mathrm{M}_{23}$.

Finally, the same analysis is performed for measurements of zone $\mathrm{M}_{23}$. The value of the mean $X P D$ in that case, is around $7 \mathrm{~dB}$ for $X P D_{V}$ and around $5.6 \mathrm{~dB}$ for $X P D_{H}$ with a $1.1 \mathrm{~dB}$ standard deviation as presented in Table 3.4. A severe decrement of the mean XPD from zone $\mathrm{M}_{21}+\mathrm{M}_{22}$ to $\mathrm{M}_{23}$ is observed, while no significant variation of the $X P D_{V}$ is appreciated. Considering that crosspolar polarizations always experience higher losses than co-polar ones, this decrement of the $X P D_{H}$ implies a decrease of the difference between the path loss of the $\mathrm{HH}$ polarization compared to that of the $\mathrm{HV}$ from zone $\mathrm{M}_{21}+\mathrm{M}_{22}$ to zone $\mathrm{M}_{23}$. This effect is mainly due to the depolarization of the radio waves because of the difference between the $\operatorname{LoS}\left(\mathrm{M}_{21}+\mathrm{M}_{22}\right)$ and $\operatorname{NLoS}\left(\mathrm{M}_{23}\right)$ scenarios. However, this depolarization is not so appreciable for $\mathrm{VV}-\mathrm{VH}$ configurations.

\section{B. Analysis of the XPD in $500 \mathrm{MHz}$ Sliding-Window Intervals}

The variation of the energy XPD (directly related with path loss) with the central frequency is also analyzed in [Malik, 2008a]. In this case, the author analyzes the XPD directly in the frequency domain from each point of the frequency response. Thus, the large amounts of points available results in a high standard deviation. The author also states that the mean XPD in frequency domains does not reveal any discernible trend.

Next, the performance of cross-polar polarizations with frequency is studied by means of the analysis of the XPD. In this case, this analysis is also carried out in $500 \mathrm{MHz}$ intervals. The mean and the standard deviation of the XPD computed in $500 \mathrm{MHz}$ sliding-window frequency intervals have been deduced from equations (3.8) and (3.9). The path loss is computed again by applying (3.4), which leads to a dramatic decrement in the number of points available compared with [Malik, 2008a (in our case with a ratio of 801:1). The results of this study are summarized in Table 3.5 and Table 3.6

The mean XPD can be considered as nearly constant with frequency in both cases and environments. Moreover, as in the case of the $3 \mathrm{GHz}$ bandwidth, $X P D_{H}>X P D_{V}$ in zone $\mathrm{M}_{21}+\mathrm{M}_{22}$ and $X P D_{V}>X P D_{H}$ in zone $M_{23}$. The standard deviation of the XPD is higher than when considering the $3 \mathrm{GHz}$ bandwidth. This effect maybe is due to the fact that the decrease in delay 


\begin{tabular}{|c|c|c|c|c|}
\hline & \multicolumn{4}{|c|}{ Zone $\mathbf{M}_{21}-\mathbf{M}_{22}$} \\
\hline & \multicolumn{2}{|c|}{$X P D_{V}$} & \multicolumn{2}{|c|}{$X P D_{H}$} \\
\hline & Mean (dB) & Std (dB) & $\overline{\text { Mean (dB) }}$ & Std (dB) \\
\hline $2.5 \mathrm{GHz}$ & 6.5 & 2.9 & 7.9 & 2.4 \\
\hline $3.0 \mathrm{GHz}$ & 5.8 & 2.9 & 6.0 & 2.4 \\
\hline $3.5 \mathrm{GHz}$ & 8.0 & 2.8 & 9.0 & 3.0 \\
\hline $4.0 \mathrm{GHz}$ & 8.0 & 2.8 & 10.7 & 3.4 \\
\hline $4.5 \mathrm{GHz}$ & 6.3 & 3.1 & 7.8 & 4.5 \\
\hline
\end{tabular}

Table 3.5. Mean and standard deviation of the $X P D$ as a function of the central frequency $(\mathrm{GHz})$ in $\mathrm{M}_{21}+\mathrm{M}_{22}$ zone.

\begin{tabular}{||c|cc|cc||}
\hline \hline \multirow{2}{*}{} & \multicolumn{4}{|c||}{ Zone $\mathbf{M}_{\mathbf{2 3}}$} \\
& \multicolumn{2}{|c|}{$\boldsymbol{X P D _ { V }}$} & \multicolumn{2}{c||}{$\boldsymbol{X P D}_{\boldsymbol{H}}$} \\
\cline { 2 - 5 } & Mean (dB) & Std (dB) & Mean (dB) & Std (dB) \\
\hline $\mathbf{2 . 5} \mathrm{GHz}$ & 6.9 & 1.6 & 6.4 & 1.4 \\
\hline $\mathbf{3 . 0} \mathrm{GHz}$ & 6.6 & 1.5 & 3.9 & 1.2 \\
\hline $\mathbf{3 . 5} \mathrm{GHz}$ & 8.0 & 1.5 & 4.6 & 1.6 \\
\hline $\mathbf{4 . 0} \mathrm{GHz}$ & 7.8 & 1.8 & 6.4 & 2.1 \\
\hline $\mathbf{4 . 5} \mathrm{GHz}$ & 5.6 & 1.2 & 4.6 & 1.4 \\
\hline \hline
\end{tabular}

Table 3.6. Mean and standard deviation of the XPD as a function of the central frequency $(\mathrm{GHz})$ in $\mathrm{M}_{23}$ zone.

resolution leads to more severe fading for each tap (due to the higher amount of multipath within one tap), thereby increasing the standard deviation of the XPD.

\subsubsection{Through-the-wall Propagation in Labs}

In this section, the group of measurements $\mathrm{M}_{1}$ is analyzed. The measurements have been undertaken in an indoor scenario where the transmitter is in LoS with the receiver, located in the same room, only for the first two positions. Then, the receiver is located out of the view of the receiver (NLoS). Therefore, the transmitted signal should go through several walls, which are made of plasterboard. This material provides a lower attenuation of the signal than bricks.

Firstly, VV and $\mathrm{HH}$ polarizations are both studied in the $3 \mathrm{GHz}$ bandwidth as a function of distance. Again, the effect of frequency is studied by varying the central frequency of a $500 \mathrm{MHz}$ sliding-window frequency interval, in order to obtain a continuous response in frequency. Lastly, as was performed previously, $\mathrm{VH}$ and $\mathrm{HV}$ configurations will be studied by also analyzing the XPD in the space and frequency domain. 


\subsubsection{Analysis of Co-polar Polarizations}

\section{A. Path Loss Modeling in $3 \mathrm{GHz}$ Bandwidth}

In Figure 3.8 [Vera Garre, 2011], the averaged path loss is plotted for each receiving position from the group of measurements $\mathrm{M}_{1}$ when transmitting TX-Loc1 (Figure 3.2) for all combinations of polarizations.

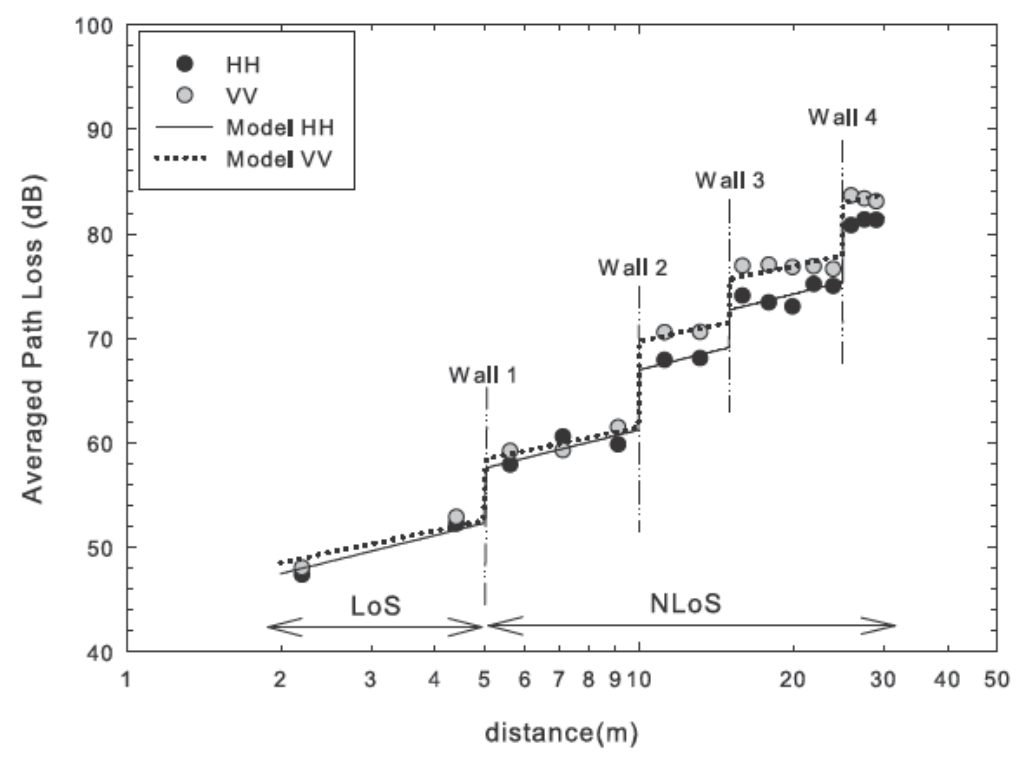

Figure 3.8. Averaged path loss for TX-Loc1 and $\mathrm{M}_{1}$ and the adjusted model.

In this environment, the path loss not only increases with distance, but also extra-losses due to each wall penetrated are also experienced. In this case, a partition loss model [Rappaport, 1996] is proposed to predict the path loss as:

$$
L(d)=L_{0}+10 n \log _{10}(d)+\sum_{i} L_{W a l l(i)}+X_{\sigma_{1}}
$$

where $L_{0}$ is the path loss at a reference distance $(1 \mathrm{~m}), n$ is the decay factor, $L_{\text {Wall (i) }}$ is the attenuation of the ith wall, and $d$ is the distance between the transmitter and receiver.

The model parameters have been extracted by applying a multi-dimensional nonlinear minimization based on the Nelder-Mead simplex algorithm as described by Lagarias et al. in [Lagarias et al., 1998], and the results are presented in Table 3.7

On the one hand, the parameter $L_{0}$ is higher for $\mathrm{VV}$ polarization as in the previous analyzed environments. On the other hand, the decay factor $n$ is higher for $\mathrm{HH}$ configuration than for the VV one. The attenuation of the walls does not seem to follow a particular behavior. 


\begin{tabular}{||c|c|c|c|c|c|c|c||}
\hline \hline & $n$ & $L_{\mathbf{0}}(\mathbf{d B})$ & $\boldsymbol{L}_{\text {wall1 }}(\mathbf{d B})$ & $\boldsymbol{L}_{\text {wall2 }}(\mathbf{d B})$ & $\boldsymbol{L}_{\text {wall3 }}(\mathbf{d B})$ & $\boldsymbol{L}_{\text {wall4 }}(\mathrm{dB})$ & $\sigma \mathbf{( d B})$ \\
\hline $\mathbf{V V}$ & 0.92 & 45.04 & 5.77 & 8.36 & 4.39 & 5.48 & 1.24 \\
\hline $\mathbf{H H}$ & 1.17 & 43.14 & 5.12 & 5.78 & 3.71 & 5.40 & 1.38 \\
\hline \hline
\end{tabular}

Table 3.7. Whole bandwidth parameters of the proposed model for $\mathrm{M}_{1}$ zone.

It can be observed how the decay factor $n$ is in the order of values provided for a one-slope path loss model in $\mathrm{M}_{21}+\mathrm{M}_{22}$ in LoS. This is due to the fact that the partition loss model here proposed can be considered as a one-slope path loss model in LoS plus the extra losses due to the four walls. In other words, the losses due to NLoS situation are included only in the extra losses of the walls' rooms.

If the one-slope model proposed in (3.5) for LoS had been considered in this environment, as Choi et al. did in [Choi et al., 2009] also for NLoS, the decay factor would have been considerably higher as it is shown in Table 3.8 .

\begin{tabular}{||c|c|c||}
\hline \hline & $n$ & $L_{0}(\mathbf{d B})$ \\
\hline VV & 3.36 & 32.22 \\
\hline HH & 3.14 & 32.85 \\
\hline \hline
\end{tabular}

Table 3.8. Whole bandwidth parameters for a one-slope model for $\mathrm{M}_{1}$ zone.

\section{B. Path Loss Modeling in $500 \mathrm{MHz}$ Sliding-Window Intervals}

As for the scenarios $\mathrm{M}_{21}+\mathrm{M}_{22}$ and $\mathrm{M}_{23}$ in Section 3.3.1.1, the influence of frequency on path loss is also analyzed from measurements of zone $\mathrm{M}_{1}$ by considering measurements in $500 \mathrm{MHz}$ sliding-window intervals. By repeating the same analysis in this scenario, the same behavior of the parameters $L_{0}$ and $n$ has been found. The value of the attenuations of the fourth walls, $L_{\text {Wall(i) }}$, also behaves approximately constant with frequency.

\subsubsection{Analysis of Cross-polar Polarizations}

Finally, the behavior of cross-polar polarizations in this scenario is compared with the behavior in the scenario previously considered. Again the analysis of the XPD is carried out in the space domain as well as in frequency domain.

\section{A. Analysis of the XPD in a $3 \mathrm{GHz}$ Bandwidth}

First, both $X P D_{V}$ and $X P D_{H}$ over the whole bandwidth have been computed for each pair of transmitting-receiving elements $\left(4 \times 4\right.$ in total) in all positions of the group of measurements $\mathrm{M}_{1}$. 
As in the case of the $\mathrm{M}_{23}$ scenario, in this case the radio waves are also depolarized when they are propagated through the walls. Hence, the XPD clearly decreases with distance, as shown in Figure 3.9

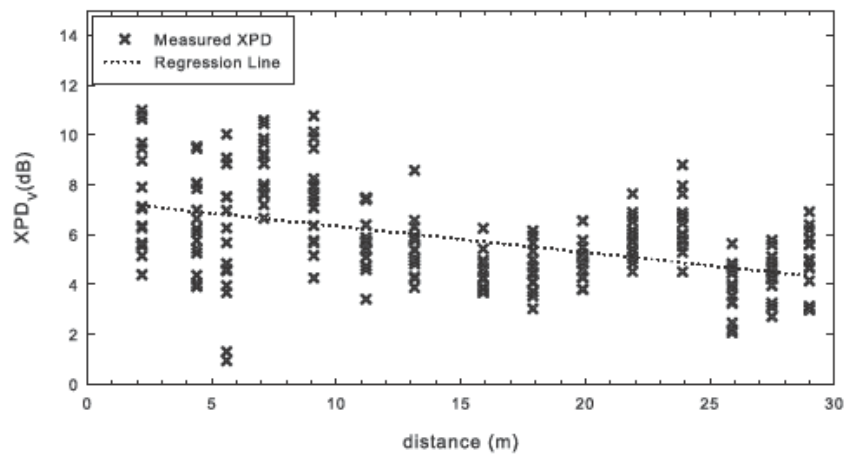

(a)

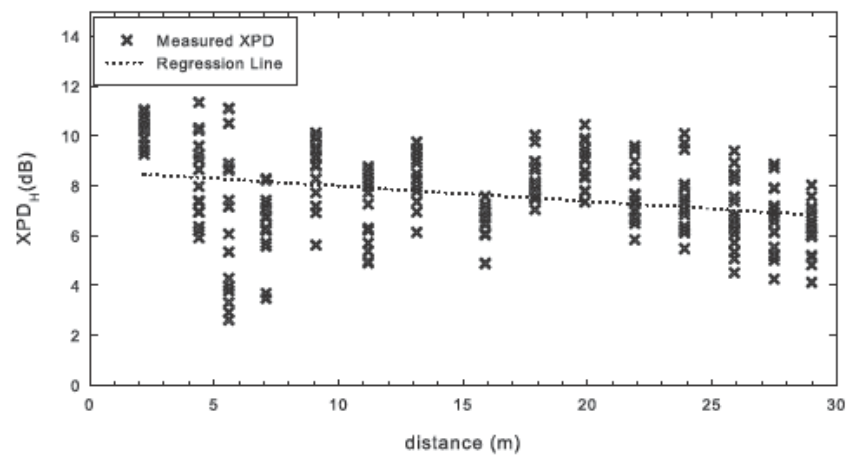

(b)

Figure 3.9. $X P D$ as function of distance for each TX-RX pair: (a) $X P D_{V}$ and (b) $X P D_{H}$.

The decrease with distance of the XPD provided by the regression line is $11.5 \mathrm{~dB} / 100 \mathrm{~m}$ for $X P D_{V}$ and $6.8 \mathrm{~dB} / 100 \mathrm{~m}$ for $X P D_{H}$. Therefore, the depolarization of the radio waves with distance is once again more pronounced for the VV configuration. Moreover, this depolarization of the transmitted signal is more pronounced here than in the corridor $\left(\mathrm{M}_{21}+\mathrm{M}_{22}\right)$.

Table 3.9 presents the mean and standard deviation of the XPD, which is found to be $5.8 \mathrm{~dB}$ for the mean $X P D_{V}$ and $7.6 \mathrm{~dB}$ for the mean $X P D_{H}$ with around $2.0 \mathrm{~dB}$ standard deviation in both cases. In that case, the $X P D_{H}$ is higher than the $X P D_{V}$, as in the corridor scenario.

\begin{tabular}{||l|cc||}
\hline \hline & \multicolumn{2}{|c|}{ Zone $\mathbf{M}_{21}-\mathbf{M}_{22}$} \\
& Mean (dB) & Std (dB) \\
\hline$X P D_{V}$ & 5.8 & 2.0 \\
\hline$X P D_{H}$ & 7.6 & 1.9 \\
\hline \hline
\end{tabular}

Table 3.9. Mean and standard deviation of the XPD in zone $\mathrm{M}_{1}$.

\section{B. Analysis of the XPD in $500 \mathrm{MHz}$ Sliding-Window Intervals}

To conclude this section, $\mathrm{VH}$ and $\mathrm{HV}$ array configurations are studied in the frequency domain by carrying out an analysis of the XPD in the frequency domain, as was performed previously for the group of measurements $\mathrm{M}_{21}+\mathrm{M}_{22}$ and $\mathrm{M}_{23}$. The results are summarized in the Table 3.10

As occurred in the group of measurements $\mathrm{M}_{21}+\mathrm{M}_{22}$, both the mean $X P D_{V}$ and the mean $X P D_{H}$ are constant with frequency. Again, the relationship between $X P D_{V}$ and $X P D_{H}$ is the same as in the case of the analysis of the whole bandwidth, i.e. $X P D_{H}>X P D_{V}$. 


\begin{tabular}{||c|cc|cc||}
\hline \hline \multirow{2}{*}{} & \multicolumn{4}{|c||}{ Zone $\mathbf{M}_{21}-\mathbf{M}_{\mathbf{2 2}}$} \\
& \multicolumn{2}{|c|}{$X P D_{V}$} & \multicolumn{2}{c||}{$X D_{H}$} \\
\cline { 2 - 5 } & Mean (dB) & Std (dB) & Mean (dB) & Std (dB) \\
\hline $\mathbf{2 . 5} \mathrm{GHz}$ & 5.9 & 2.5 & 7.2 & 2.2 \\
\hline $3.0 \mathrm{GHz}$ & 7.1 & 2.7 & 7.6 & 3.0 \\
\hline $3.5 \mathrm{GHz}$ & 7.0 & 2.8 & 8.4 & 3.1 \\
\hline $\mathbf{4 . 0} \mathrm{GHz}$ & 5.9 & 3.0 & 7.6 & 2.9 \\
\hline $\mathbf{4 . 5} \mathrm{GHz}$ & 5.1 & 2.8 & 7.1 & 3.0 \\
\hline \hline
\end{tabular}

Table 3.10. Mean and standard deviation of the $X P D$ as a function of the central frequency $(\mathrm{GHz})$ in zone $\mathrm{M}_{1}$.

As in zones $\mathrm{M}_{21}+\mathrm{M}_{22}$ and $\mathrm{M}_{23}$, an increase of the standard deviation of the XPD is observed when studying the influence of frequency in this parameter. This behavior is due to a more severe fading for each tap caused by a decrease in the delay resolution.

\subsection{Delay Dispersion}

The delay dispersion is a crucial parameter for studying the performance of any digital wireless transmission, since it fixes the maximum data rate without equalization and gives us an idea of the flatness of the channel in frequency [Rappaport, 1996].

The RMS delay spread can be computed from the PDP as its second moment (see Appendix B, Section B.5.2):

$$
\sigma_{\tau}=\sqrt{\frac{\sum_{k} P\left(\tau_{k}\right) \tau_{k}^{2}}{\sum_{k} P\left(\tau_{k}\right)}-\left(\frac{\sum_{k} P\left(\tau_{k}\right) \tau_{k}}{\sum_{k} P\left(\tau_{k}\right)}\right)^{2}}
$$

where $P\left(\tau_{k}\right)$ is the received power (in linear units) at $\tau_{k}$.

As mentioned at the beginning of Section 3.3 , the PDP can be calculated from the square of the channel impulse response by applying equation (3.3). In our measurement campaign, $4 \times 4$ PDPs are available at each position in all scenarios. Furthermore, only those PDPs which guarantee that at least the $85 \%$ of their points in the frequency domain are $10 \mathrm{~dB}$ over the noise level have been selected.

In the following sections, the RMS delay spread is analyzed in all the scenarios considered, and the results are compared with those obtained from the $200 \mathrm{MHz}$ bandwidth in [Molina-Garcia-Pardo et al., 2008c]. The same threshold of $10 \mathrm{~dB}$ is used in both analyses for a fair comparison of the results. 


\subsubsection{Delay Dispersion in LoS and NLoS along the Corridor}

\subsubsection{Analysis in Space Domain}

First, the results of the group of measurements $\mathrm{M}_{21}, \mathrm{M}_{22}$, and $\mathrm{M}_{23}$ when the transmitter is located in TX-Loc2 are analyzed. In this case, the transmitter and receiver are in LoS conditions for the group of measurements $\mathrm{M}_{21}$ and $\mathrm{M}_{22}$, and in NLoS for $\mathrm{M}_{23}$.

Figure 3.10 shows that the RMS delay spread is approximately constant with distance in zone $\mathrm{M}_{21}$, the mean value of delay spread being in the order of $3 \mathrm{~ns}$ in this zone for all polarizations. Such low delay dispersion is due to the guiding effect caused by the corridor. In contrast, in zone $\mathrm{M}_{22}$, the delay dispersion experiences a gradual increase after the $\mathrm{T}$ junction until the end of the corridor due to the multiple reflections caused by the end of the main corridor and the corridor to the left. Thus, the mean RMS delay spread in zone $\mathrm{M}_{22}$ is around $9 \mathrm{~ns}$ for co-polar polarizations and about $6 \mathrm{~ns}$ for the cross-polar ones.

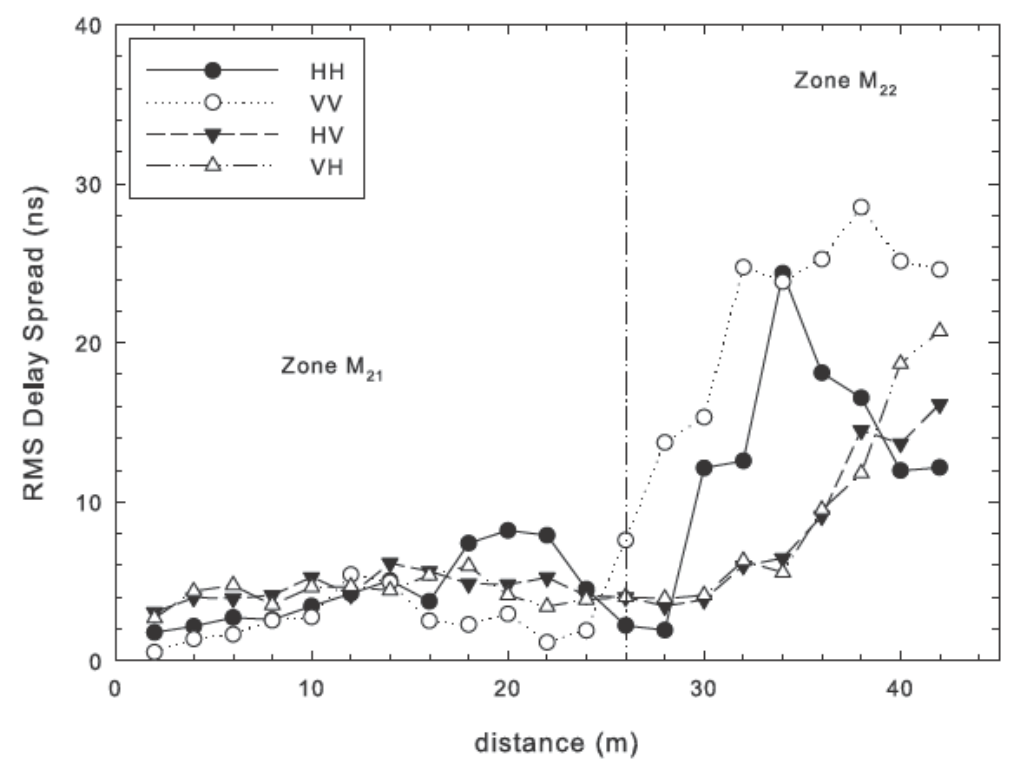

Figure 3.10. RMS delay spread for the group of measurements $\mathrm{M}_{21}$ and $\mathrm{M}_{22}$ (corridor in LoS).

These values are significantly lower than those provided for the same environments in [Molina-Garcia-Pardo et al., 2008c] for the $200 \mathrm{MHz}$ bandwidth because of the increase in the delay resolution from $0.33 \mathrm{~ns}$ for the $3 \mathrm{GHz}$ bandwidth to $5 \mathrm{~ns}$ for the $200 \mathrm{MHz}$ bandwidth.

Next, the behavior of the RMS delay spread when turning left at the T junction (group of measurements $\mathrm{M}_{23}$ ) is considered. In this case, the receiver is always situated in NLoS and any of the $4 \times 4$ PDPs available at each position meet the requirements of having at least $85 \%$ of its frequency points over the noise level. Therefore, the RMS delay spread in this zone has not been computed. 


\subsubsection{Analysis in Frequency Domain}

As previously mentioned, the bandwidth in which the delay dispersion is analyzed changes the delay resolution of the power delay profile, and therefore the computed RMS delay spread. Hence, the higher the bandwidth, the lower the delay resolution, and consequently the lower the delay dispersion.

Nevertheless, the influence of frequency on delay dispersion can be observed by fixing the bandwidth and varying the central frequency. In line with the analysis performed for path loss in the previous section, a bandwidth of $500 \mathrm{MHz}$ is chosen. Therefore, in this case the delay resolution is 2 $\mathrm{ns}$, and the central frequency varies from 2.25 to $4.75 \mathrm{GHz}$.

Figure 3.11 shows the RMS delay spread in the frequency domain computed by averaging all the positions of the group of measurements $\mathrm{M}_{21}$ in the space domain. The delay dispersion remains constant with frequency around $5 \mathrm{~ns}$. As expected, the values of the RMS delay spread are higher than in the case of the $3 \mathrm{GHz}$ bandwidth (about 1 or $2 \mathrm{~ns}$ in those positions) because of the reduction of delay resolution.

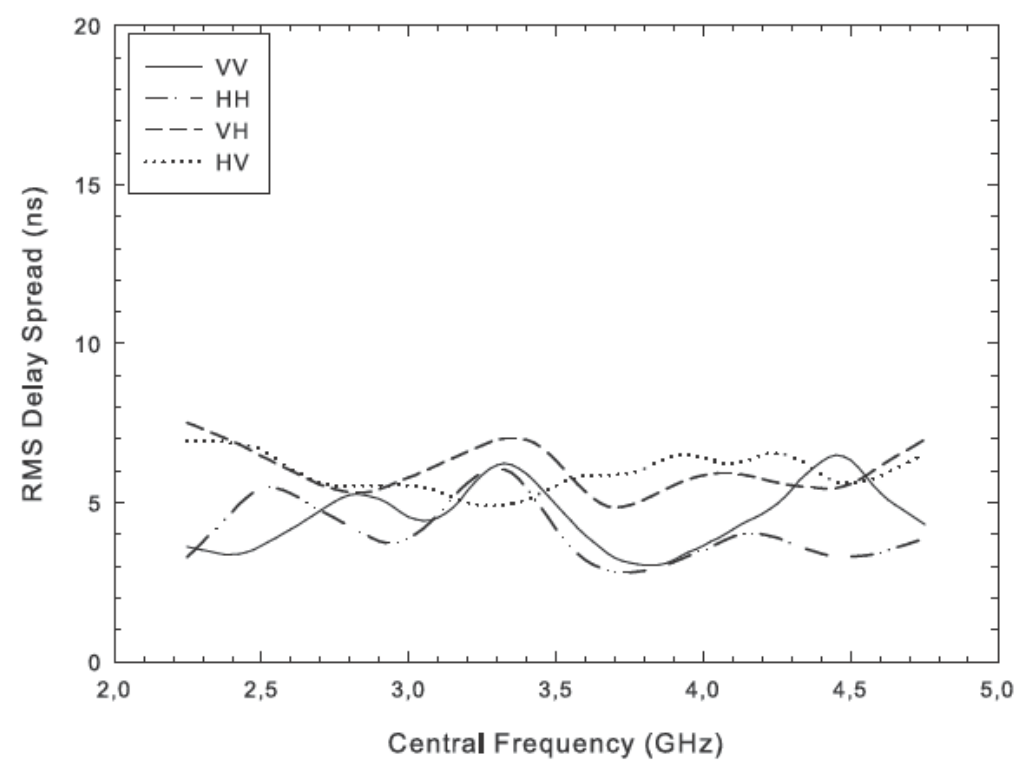

Figure 3.11. RMS delay spread as a function of the central frequency for zone $\mathrm{M}_{21}$ (corridor in LoS).

In the zone $\mathrm{M}_{22}$ and $\mathrm{M}_{23}$, the same analysis has been performed and the same behavior has been observed. Nevertheless, the low amount of valid PDPs does not provide significant information. 


\subsubsection{Delay Dispersion in Labs}

\subsubsection{Analysis in Space Domain}

Finally, the RMS delay spread for the group of measurements $\mathrm{M}_{1}$ is analyzed. Figure 3.12 shows how the RMS delay spread tends to increase with distance and number of walls penetrated. In general, co-polar polarizations have less delay spread than the cross-polar configuration, and the $\mathrm{HH}$ polarization in particular presents the lowest delay dispersion. This effect is also found in the analysis for the $200 \mathrm{MHz}$ bandwidth [Molina-Garcia-Pardo et al., 2008c] where, for example, the RMS delay spread in LoS is $7.2 \mathrm{~ns}$ for co-polar polarizations and $10.7 \mathrm{~ns}$ for cross-polar ones.

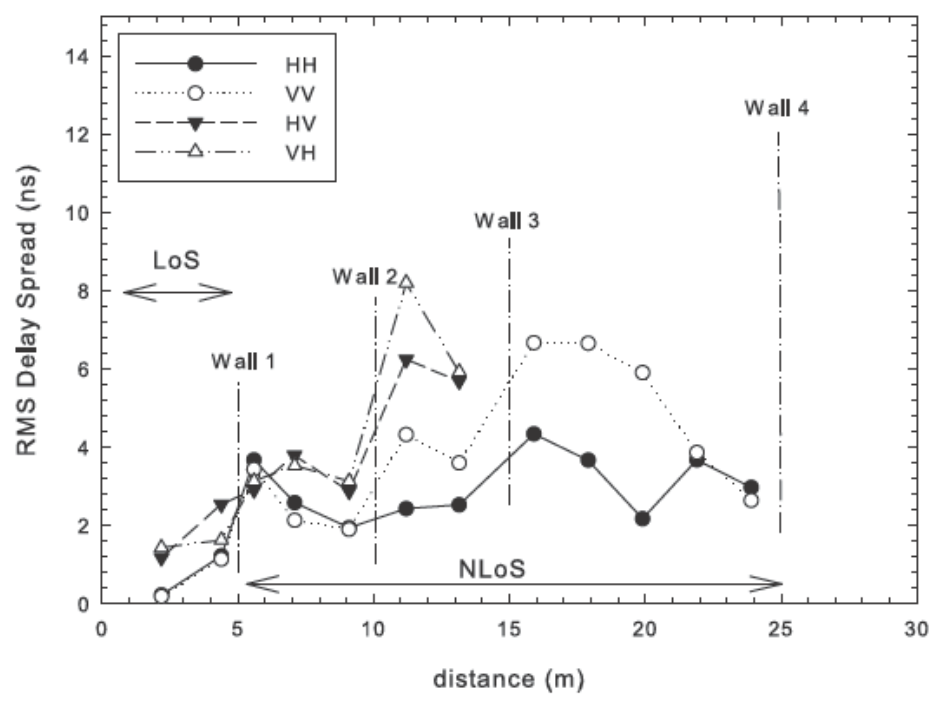

Figure 3.12. RMS delay spread for the group of measurements $M_{1}$.

The mean RMS delay spread has been found to be about $3 \mathrm{~ns}$ for all polarizations, taking into account the difference in the number of valid points for each polarization as shown in Figure 3.12

It also should be pointed out that after the fourth wall, for co-polar configurations any of the $4 \times 4$ PDPs are available since every position meets the requirements of having at least $85 \%$ of its frequency points over the noise level. The same situation occurs for cross-polar polarizations after the third wall.

Comparing the results obtained here for the $3 \mathrm{GHz}$ bandwidth with those obtained for the 200 MHz bandwidth in [Molina-Garcia-Pardo et al., 2008c], the same behavior of the delay spread can be observed. Again, the values of RMS delay spread were higher in the case of the $200 \mathrm{MHz}$ bandwidth, resulting in more $10 \mathrm{~ns}$ of mean RMS in all cases. 


\subsubsection{Analysis in Frequency Domain}

Finally, the analysis in the frequency domain of the RMS delay spread is repeated for the measurements in the laboratories. In Figure 3.13 the way in which the delay dispersion remains constant with frequency as in zone $\mathrm{M}_{21}$ is shown. In that case, the mean RMS delay spread is about $5 \mathrm{~ns}$ for all polarizations. Again, the decrement of delay resolution increases the mean value of RMS delay spread.

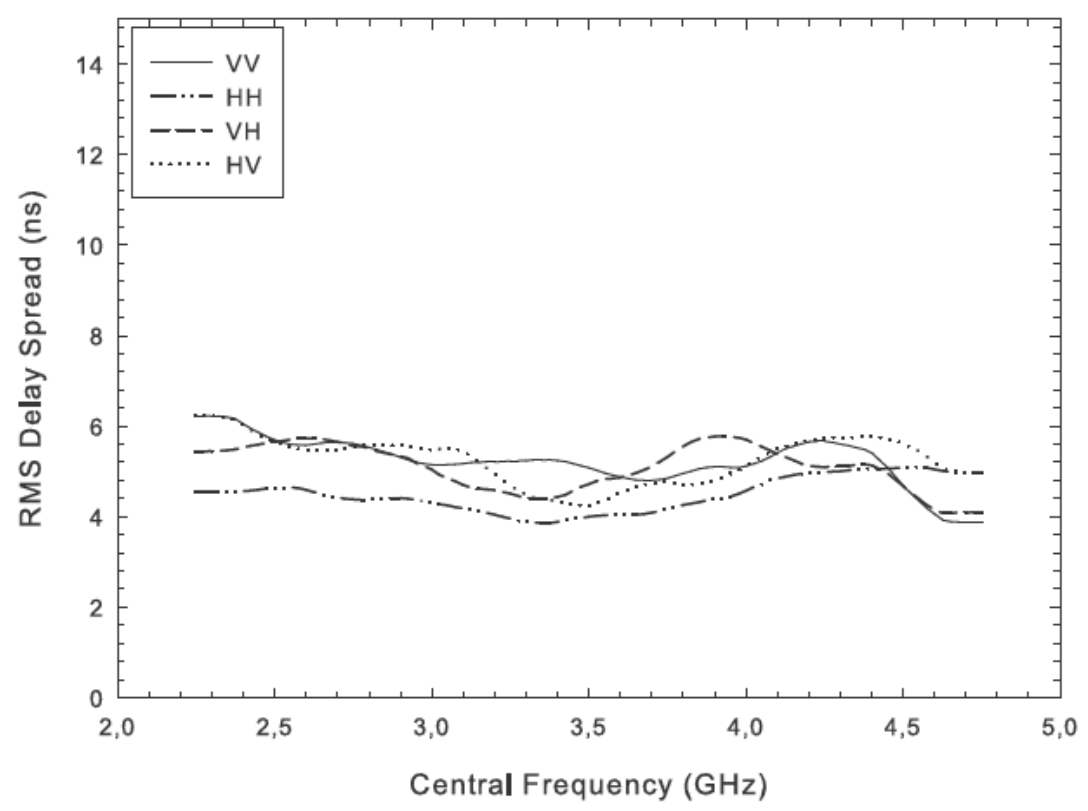

Figure 3.13. RMS delay spread as a function of the central frequency for zone $\mathrm{M}_{1}$.

\subsection{Evaluation of the MIMO System Capacity}

This section deals with multiantenna element parameters, extracted from the measured data, the $G$ matrices. In this way, the maximum theoretical capacity for an $N \times M$ MIMO system can be obtained as [Telatar, 1995], [Foschini and Gans, 1998]:

$$
C=\log _{2}\left(\operatorname{det}\left(\mathbf{I}_{\mathbf{N}}+\frac{S N R}{M} \mathbf{H H}^{\mathbf{\dagger}}\right)\right)
$$

where $\mathbf{I}_{\mathbf{N}}$ is the $N \times N$ identity matrix, $†$ represents the conjugate transpose operation, $\mathbf{H}$ is the Frobenius normalized $G$ matrix, and SNR is the signal to noise ratio at the receiver. Uniform linear arrays having four elements $(M=N=4)$ have been considered.

Attending to SNR in (3.12), two cases can be considered. Firstly, MIMO capacity is studied by assuming a constant received power. On the one hand, it can be considered a receiver which adjusts 
the received power using an automatic gain control (CAG) amplifier, as it is used in third generation (3G) systems to control the received power and decrease the interference. This implies the use of a constant SNR in all positions independently of the received power, and the effect of spatial richness is directly observed in the capacity curves.

On the other hand, one can think in a system where the transmitted power is fixed and the SNR at the receiver is mainly determined by the path loss. In this case, capacity shows both effects related to the received power and the spatial richness.

Moreover, the correlation independently at both the transmitter and the receiver [Eggers et al., 1993] has been studied. The complex correlation coefficient at the frequency $f$ for the kth time-realization between the reception antennas $p$ and $q$ can be computed as [Lienard et al., 2003]:

$$
\rho^{p, q}(f, k)=\frac{E\left[\overline{h^{p}(f, k)} \cdot \overline{h^{q}(f, k)}{ }^{*}\right]}{\sqrt{E\left[\left|\overline{h^{p}(f, k)}\right|^{2} \cdot\left|\overline{h^{q}(f, k)}\right|^{2}\right]}}
$$

where $E[]$ is the expectation operation value for all the transmitting element antennas, and $\overline{h^{p}(f, k)}$ is the zero mean pth row of the $\mathrm{H}$ matrix. The correlation coefficient between the transmitting antennas can be calculated in a similar way by swapping the rows and the columns on the matrix. For more information about MIMO and/or the correlation, the reader can refer to Appendix $\mathrm{A}$

The study of capacity in different environments has been divided into two cases: constant received power and constant transmitted power. The results are presented below.

\subsubsection{Capacity for Constant Received power}

\subsubsection{Capacity along the Corridor in LoS}

Firstly, the capacity performance in a corridor environment for the group of measurements $\mathrm{M}_{21}+\mathrm{M}_{22}$ in LoS is studied. To accomplish this, some significant positions of the receiver have been chosen: two before the $\mathrm{T}$ junction, the third in the $\mathrm{T}$ junction, and the last one at the end of the main corridor, that is, when the receiver is in the positions 1, 6, 13, and 21 of $\mathrm{M}_{21}+\mathrm{M}_{22}$. For each polarization and position 801 points are available.

The CCDF (Complementary Cumulative Distribution Function) of the normalized capacity (for an SNR of $10 \mathrm{~dB}$ ) for all polarizations depicted in Figure 3.14. For a probability of 90\%, Table 3.11 summarizes such results. 

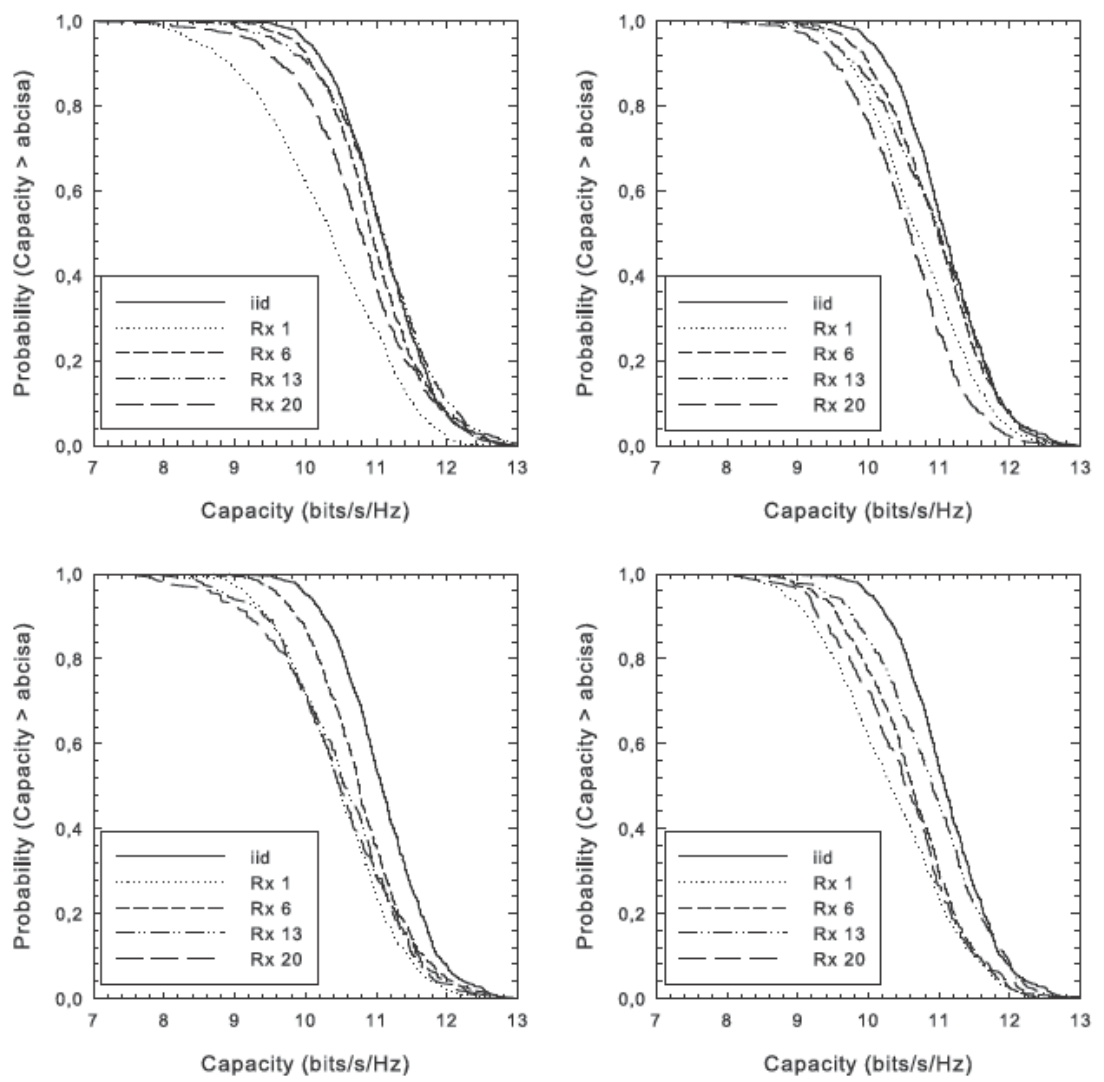

Figure 3.14. CCDFs of the capacity for the group of measurements $M_{21}+M_{22}$ for different polarizations.

\begin{tabular}{|c|c|c|c|c|}
\hline & & Tx Correlation & Rx Correlation & Capacity $(90 \%)$ \\
\hline \multirow{4}{*}{$\operatorname{Rx} 1$} & HH & 0.55 & 0.56 & 8.9 \\
\hline & VV & 0.49 & 0.49 & 9.7 \\
\hline & HV & 0.54 & 0.54 & 9.4 \\
\hline & VH & 0.56 & 0.55 & 9.1 \\
\hline \multirow{4}{*}{ Rx 6} & HH & 0.48 & 0.48 & 10.1 \\
\hline & VV & 0.47 & 0.47 & 10.0 \\
\hline & HV & 0.50 & 0.49 & 9.8 \\
\hline & VH & 0.51 & 0.51 & 9.6 \\
\hline \multirow{4}{*}{$\operatorname{Rx} 13$} & HH & 0.45 & 0.46 & 10.1 \\
\hline & VV & 0.48 & 0.48 & 10.2 \\
\hline & HV & 0.52 & 0.52 & 9.4 \\
\hline & VH & 0.48 & 0.48 & 9.8 \\
\hline \multirow{4}{*}{$\operatorname{Rx} 13$} & HH & 0.49 & 0.50 & 9.6 \\
\hline & VV & 0.52 & 0.52 & 9.6 \\
\hline & HV & 0.52 & 0.52 & 9.2 \\
\hline & VH & 0.52 & 0.52 & 9.3 \\
\hline
\end{tabular}

Table 3.11. Correlations and capacity for SNR fixed at $10 \mathrm{~dB}$ when probability $>0.9$ in corridor scenario $\left(\mathrm{M}_{21}+\mathrm{M}_{22}\right)$. 
In this scenario, the co-polar configurations outperform the cross-polar ones. The same effect was observed in LoS for $200 \mathrm{MHz}$ bandwidth [Molina-Garcia-Pardo et al., 2008c]. The highest capacity is provided in the position next to the $\mathrm{T}$ junction (position $3, \mathrm{Rx} 3=26 \mathrm{~m}$ ), where the lowest correlation coefficient is also given. This behavior is a consequence of the multiple reflections coming from the corridor on the left.

Furthermore, the capacity for $\mathrm{HH}, \mathrm{VV}, \mathrm{HV}$, and $\mathrm{VH}$ at the end of the corridor tends to converge due to a depolarization of the radio waves along the corridor.

\subsubsection{Capacity along the Laboratories}

Next, the capacity for constant received power in the laboratories conditions will be studied by analyzing the group of measurements $\mathrm{M}_{1}$. Again, the CCDFs of capacity in each laboratory (room) have been computed and are shown in Figure 3.15 In each room, the number of points available for the analysis is $801 \times$ number of positions measured in the room. The results are summarized in Table 3.12 for a probability of $90 \%$. In Room 1 the receiver is in LoS with the transmitter, while in Rooms 2 and 3 the receiver is in NLoS.
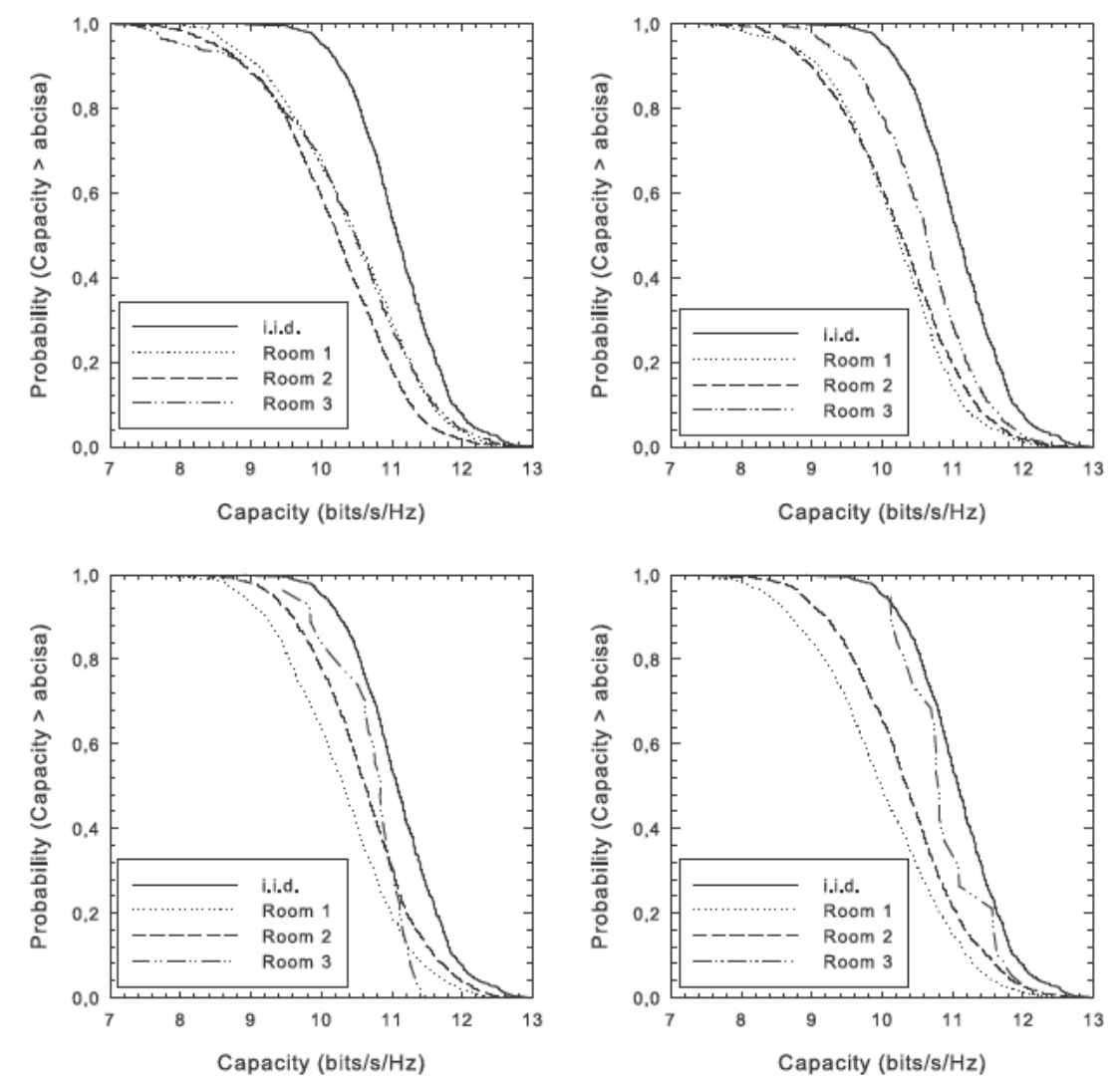

Figure 3.15. CCDFs of the capacity for the group of measurements $\mathrm{M}_{1}$ for different polarizations. 


\begin{tabular}{||c|c|c|c|c||}
\hline \hline \multicolumn{2}{||c|}{} & Tx Correlation & Rx Correlation & Capacity (90\%) \\
\hline \multirow{4}{*}{ Room 1 } & HH & 0.55 & 0.56 & 9.1 \\
& VV & 0.56 & 0.57 & 9.1 \\
& HV & 0.57 & 0.56 & 9.2 \\
& VH & 0.59 & 0.62 & 8.7 \\
\hline \multirow{4}{*}{ Room 2 } & HH & 0.55 & 0.58 & 8.9 \\
& VV & 0.59 & 0.57 & 9.0 \\
& HV & 0.52 & 0.51 & 9.5 \\
& VH & 0.54 & 0.55 & 9.2 \\
\hline \multirow{4}{*}{ Room 3 } & HH & 0.55 & 0.56 & 8.9 \\
& VV & 0.52 & 0.52 & 9.6 \\
& HV & 0.48 & 0.48 & 9.8 \\
& VH & 0.46 & 0.46 & 10.1 \\
\hline \hline
\end{tabular}

Table 3.12. Correlations and capacity for SNR fixed at $10 \mathrm{~dB}$ when probability $>0.9$ in laboratories scenario $\left(\mathrm{M}_{1}\right)$.

It should be noted that after the second and third walls only some points of $\mathrm{HH}$ and VV polarizations meet the SNR requirements described at the beginning of this section. Therefore, results for those points are not presented.

The way in which capacity increases with the number of walls penetrated can be observed. Thus, the highest values of capacity correspond to the furthest laboratory (room 3) for all polarizations. Consequently, this performance also corresponds to a decrease in the correlation coefficient with distance. Besides, the cross-polar polarizations present higher capacity than co-polar ones, in contrast to the performance in $\operatorname{LoS}\left(\mathrm{M}_{21}+\mathrm{M}_{22}\right)$. This also agrees with the results provided in [Molina-Garcia-Pardo et al., 2008c] for $200 \mathrm{MHz}$ bandwidth, where in NLoS cross-polar polarizations outperform the co-polar configuration.

\subsubsection{Capacity for Constant Transmitted power}

\subsubsection{Capacity along the Corridor in LoS}

Next, the performance of the capacity is studied in a corridor environment for the group of measurements $\mathrm{M}_{21}+\mathrm{M}_{22}$ in the case of constant transmitted power, that is, in the case of using the measured SNR in the receiver. To fairly compare the performance of the capacity for the three groups of measurements, the measured SNR at every point has been referred to the measured SNR at the end of the corridor (last position of $\mathrm{M}_{22}$ ), where the lowest value of received power was found. An extra threshold of $10 \mathrm{~dB}$ above such a level was also added.

It should also be pointed out that in this case, the mean capacity has been computed by averaging the capacity over all the measured frequency band (801 frequency points in $3 \mathrm{GHz}$ bandwidth). 
The way in which capacity decreases exponentially with distance as SNR does can be observed in Figure 3.16 In this case, the effect of the SNR is much higher than that which comes from the correlation (as in the previous section). In this scenario, capacity is higher for $\mathrm{HH}$ polarization although capacity for all configurations tends to converge at the end of the corridor.

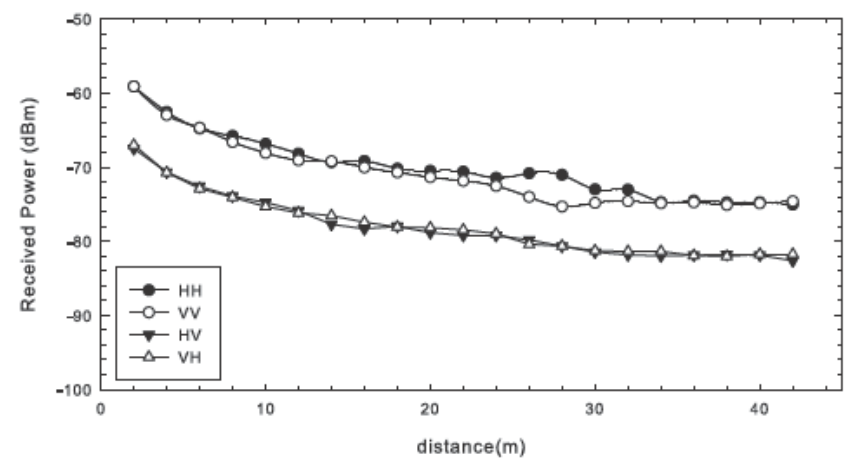

(a)

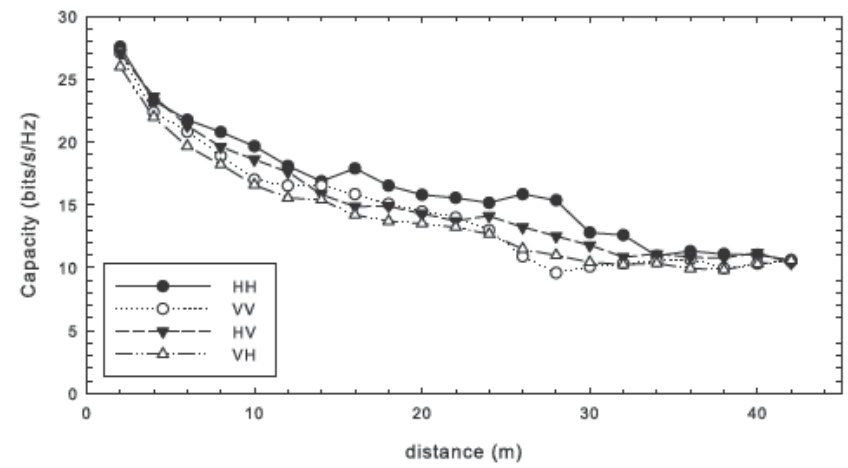

(b)

Figure 3.16. (a) Received power and (b) the mean measured capacity as a function of distance for a variable SNR in the main corridor $\left(\mathrm{M}_{21}+\mathrm{M}_{22}\right)$ in LoS.

\subsubsection{Capacity across the Laboratories}

Secondly, the capacity for constant transmitted power (SNR variable) will be analyzed. In this case, the variable SNR is referred to the measured SNR at the last laboratory. In Figure 3.17 the capacity for the group of measurements $\mathrm{M}_{1}$ is depicted. After the second and third walls only some points of $\mathrm{HH}$ and VV polarizations meet the SNR requirements, so results for those points are not presented.

Since SNR decreases in the same manner as path loss, capacity decreases with distance and exhibits strong decrements after each wall. Again, this performance of the capacity was observed for $200 \mathrm{MHz}$ bandwidth in [Molina-Garcia-Pardo et al., 2008c]. 


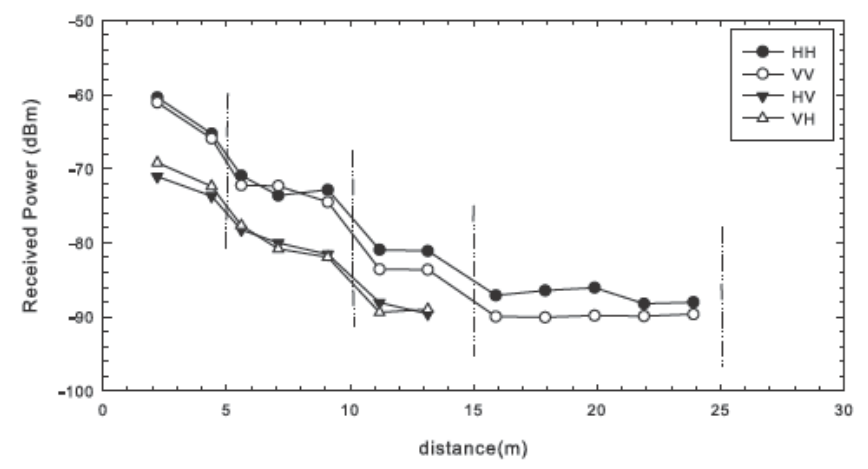

(a)

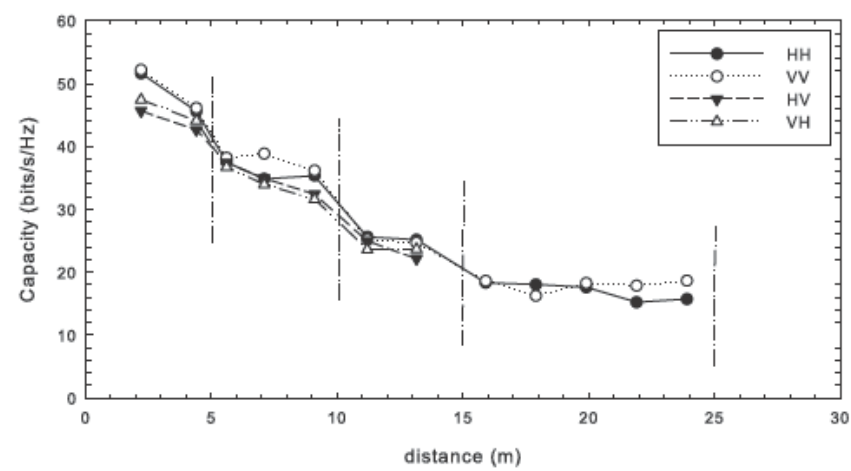

(b)

Figure 3.17. (a) Received power and (b) the mean measured capacity as a function of distance for a variable SNR in the labotories $\left(\mathrm{M}_{1}\right)$.

\subsection{Conclusions}

In this chapter, the results from an indoor $4 \times 4 \mathrm{MIMO}$ measurement campaign in the $2-5 \mathrm{GHz}$ UWB frequency band have been reported. Such a measurement campaign was carried out using a multiport vector network analyzer within a $3 \mathrm{GHz}$ bandwidth. Important single-antenna and multiantenna parameters such as path loss, RMS delay spread and the MIMO capacity were analyzed in different environments and for different polarizations ( $\mathrm{HH}, \mathrm{VV}, \mathrm{HV}$ and $\mathrm{VH})$.

The aim of this measurement campaign was, on the one hand, to compare the results obtained with those obtained in [Molina-Garcia-Pardo et al., 2008c] in the same environment at $2.45 \mathrm{GHz}$ for a $200 \mathrm{MHz}$ bandwidth. On the other hand, due to the huge bandwidth measured, the second objective of this work was to analyze the effect of frequency in the path loss as well as in the delay dispersion. To accomplish this, measurements have been divided into $500 \mathrm{MHz}$ sliding-window frequency intervals.

The path loss for VV and $\mathrm{HH}$ polarizations (co-polar) was analyzed by computing the mean path loss as a function of distance, in contrast with VH and $\mathrm{HV}$ polarizations (cross-polar) that were analyzed by means of the analysis of the Cross Polarization Discrimination or XPD. In all 
the scenarios considered, the path loss for co-polar configurations increases linearly with distance. In the case of the laboratories, the walls (made of plasterboard) are modeled with an extra loss. The $X P D$ in LoS in the corridor is found to be constant with distance at around $7.1 \mathrm{~dB}$ for $X P D_{V}$ and 8.1 $\mathrm{dB}$ for $X P D_{H}$. In contrast, it decreases with distance in NLoS for propagation in laboratories at 11.5 $\mathrm{dB} / 100 \mathrm{~m}$ for $X P D_{V}$ and $6.8 \mathrm{~dB} / 100 \mathrm{~m}$ for $X P D_{H}$.

In the frequency domain, the average path loss increases with frequency because of the reduction of the effective area of the antennas. The model parameters of the mean path loss as a function of distance have been determined from a $500 \mathrm{MHz}$ bandwidth and by varying the central frequency. The decay factor has been found to be constant with frequency in all environments. The losses of each wall in the $\mathrm{M}_{1}$ environment also behave constantly with frequency, as expected. The central frequency do not influence the behavior of the $X P D$, which is also constant with frequency.

Regarding delay dispersion, in zone $\mathrm{M}_{21}$ in LoS, the RMS delay spread is around $3 \mathrm{~ns}$ and is constant with distance; however it tends to increase after the $\mathrm{T}$ junction up to more than $10 \mathrm{~ns}$ at the end of the corridor. Furthermore, in the laboratories (zone $\mathrm{M}_{1}$ ) it increases with respect to the number of walls penetrated. The same behavior was found in the previous analysis with a $200 \mathrm{MHz}$ bandwidth, but in this case the lower delay resolution results in higher values of RMS delay spread.

Although the analysis in the frequency domain of delay dispersion implies an increment of the RMS delay spread caused by the narrower bandwidth used, it was shown that frequency does not have an effect on the delay dispersion.

Finally, the capacity of the $4 \times 4$ MIMO-UWB system was analyzed in different environments for different polarizations $(\mathrm{HH}, \mathrm{VV}, \mathrm{HV}$, and $\mathrm{VH})$ under two different hypotheses: the first one, in the case of constant received power (constant SNR) and the second one in the case of constant transmitted power (variable SNR).

In the case of constant SNR, the capacity for the co-polar configurations (VV and $\mathrm{HH}$ polarizations) in LoS conditions was found to be higher than for the cross-polar ones. However, the contrary behavior was found when the receiver was in NLoS with the transmitter. The same behavior was found in a similar analysis for $200 \mathrm{MHz}$ bandwidth. In the case of variable SNR, the capacity was strongly influenced by the SNR. Thus, the capacity tends to decrease as the SNR do in all the environments under analysis. 


\section{Chapter 4}

\section{MULTi-DimenSiONAL \\ CHARACTERIZATION IN TUNNELS}

In order to optimize the use of railway infrastructures, new technologies related to train-to-track or train-to-train communications have emerged. Thus, there is an increasing necessity of developing and implementing control/command equipment, signalling systems and new information and communication technologies in order to develop safe and efficient trains and infrastructures operation and management.

In order to cover the tunnel, two solutions have traditionally been proposed: the so-called "natural propagation" using antennas of small size, and leaky coaxial cables [Liénard and Degauque, 1999]. However, the implementation of radiating cables is expensive, at least in long tunnels, because the diameter of the cable must be large enough to avoid a prohibitive attenuation in the $1-5 \mathrm{GHz}$ band, which will be considered in this chapter.

This chapter is devoted to the experimental characterization of the radio channel in a special environment such as a transportation tunnel. To achieve this goal, measurements performed in a empty tunnel in the $2.8-5 \mathrm{GHz}$ Ultra-Wideband (UWB) frequency band are used. 
A brief state of the art about channel modeling in tunnels is presented in Section 4.1. Some diversity techniques that will be evaluated at the end of this chapter are also reviewed in this section

Section 4.2 details the channel sounder, the measurement environment, and the polarization of the transmitting and receiving arrays.

In Section 4.3 basic single antenna parameters are deduced from measurements such as the mean path loss, the complex correlation coefficient, the power delay profile and the RMS (Root Mean Square) delay spread, the angular power spectrum and the selectivity in frequency domain. These parameters are analyzed taking into account the behavior of the propagation channel in tunnels.

In Section 4.4, the gain provided by diversity techniques previously presented are studied in tunnels. Firstly, in Section 4.4.1 the frequency diversity domain is analyzed. Next, the effect of polarization in the Multiple-Input Multiple-Output (MIMO) system capacity is outlined in Section 4.4.2. Finally, the benefits of using Time Reversal in tunnels are evaluated in Section 4.4.3.

The main conclusions deduced from the work presented in this chapter are outlined in Section 4.5 ,

\subsection{State of the Art of Channel Modeling in Tunnels}

As in other environments, achieving a reliable communication in tunnels implies a preliminary knowledge of the propagation channel. In the following sections the theoretical characterization of the propagation in tunnels is described. Next, a review of some diversity techniques for multiantenna communications in tunnels is presented.

\subsubsection{Theoretical Characterization}

Many approaches have been developed to theoretically study electromagnetic wave propagation inside a tunnel, the most well known being those based either on the ray theory [Pallares et al., 2001] or on the modal theory [Mahmoud, 1998] [Dudley et al., 2007]. The transmitting frequency range must be chosen such that the attenuation per unit length is not prohibitive. To fulfill this requirement, the tunnel must behave as an oversized waveguide. Consequently, the wavelength must be much smaller than the transverse dimensions of the tunnel, which leads to transmitting frequencies greater than few hundred MHz in usual road or train tunnels [Molina-Garcia-Pardo et al., 2011].

The objective of this section is to outline the theoretical characterization of the radio channel in tunnels of simple geometry, such as those with a rectangular cross section as shown in Figure 4.1 . 


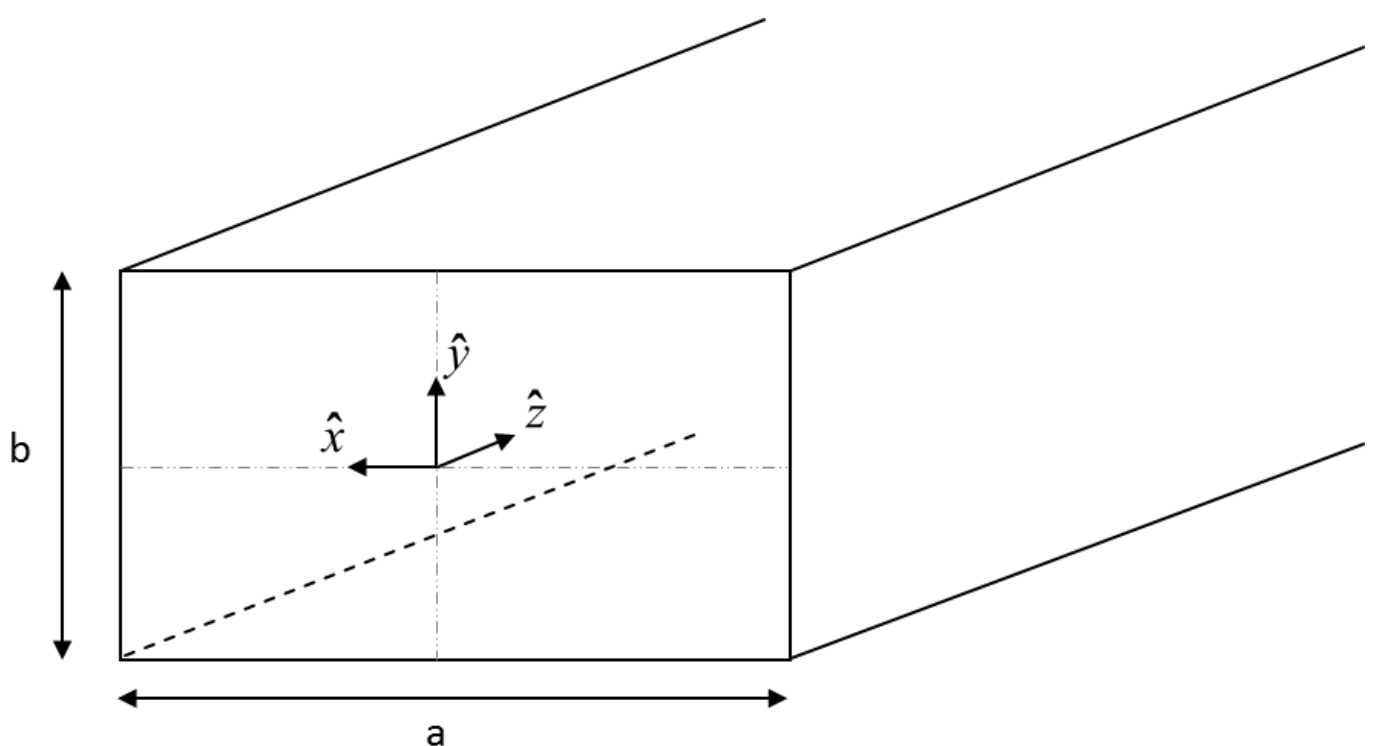

Figure 4.1. Geometry of the rectangular tunnel.

\subsubsection{Ray Tracing Approach}

Ray theory combined with image theory leads to a set of virtual transmitting (Tx) antennas. If the tunnel is of rectangular cross-section, the determination of the location of these virtual antennas is straightforward, and it is independent of the location of the receiving point. The total field is obtained by summing the contribution of all rays connecting the Tx images and the receiving point $(\mathrm{Rx})$, whilst considering the reflection coefficients on the tunnel walls. However, even by assuming ray propagation, the summation of the contribution of the rays at the Rx point must take into account the vector nature of the electric field. Before each reflection on a wall, the electric field vector must be expressed as the sum of two components: one perpendicular to the incidence plane $E_{\text {perp }}$ and one parallel to this plane $E_{\text {para }}$. To each of these components, reflection coefficients $R_{T M}$ and $R_{T E}$ are respectively applied, mathematical expressions for which can be found in any book treating electromagnetic wave propagation [Wait, 1962] [Dudley, 1994]. After each reflection, one can thus obtain the new orientation of the electric field vector. The same approach is successively applied by following the rays and by finding the successive incidence planes. Nevertheless, it has been shown [Lienard et al., 1997] that if the distance between Tx and Rx becomes greater than three times the largest dimension of the tunnel cross-section, the waves remain nearly linearly polarized. In this case, the vector summation of the electric field radiated by an antenna and its images becomes a simple scalar summation as in:

$$
E(x, y, z)=\sum_{m} \sum_{n}\left(R_{T M}\right)^{m}\left(R_{T E}\right)^{n} E_{d}\left(S_{m n}\right)
$$

where $E_{d}\left(S_{m n}\right)$ is the electric field radiated in free space by the image source $S_{m n}$ and corresponding to rays having $m$ reflections on the walls perpendicular to the Tx dipole axis and $n$ reflections on the 
walls parallel to the dipole axis [Mahmoud and Wait, 1974]. In the following examples the excitation by an electric dipole will be considered, but this is not a strong restriction in the ray approach since other kinds of antennas can be treated by introducing their free space radiation pattern into the model, i.e. by weighting the rays in a given direction by a factor proportional to the antenna gain in this direction.

Lastly, it must also be emphasized that the reflection coefficients on the walls tend to 1 if the angle of incidence on the reflecting plane tends to $90^{\circ}$. This means that, at large distances, only rays impinging the tunnel walls with a grazing angle of incidence play a leading part in the received power, thus the number of rays that fulfill this condition is important. Typically, to predict the total electric field in standard tunnels and at distances of a few hundred meters, 20 to 30 rays are needed. One should note that if a base station is located outside the tunnel, and if a mobile moves inside the tunnel, the ray theory can still be applied by taking the diffraction in the aperture plane of the tunnel into account [Mariage et al., 1994].

\subsubsection{Modal Theory Approach}

This approach is based on considering the tunnel as a waveguide where:

- The dielectric is the air.

- The dimensions of the tunnel are much larger than the wavelength of the propagated signal, $\lambda$.

The analysis based on modal theory is simple for a rectangular cross-section. However, complexity increases considerably for more complicated structures. For the shake of simplicity, in this section, the analysis for a rectangular cross-section is detailed.

The modes propagating inside the tunnel are hybrid modes $E H_{m n}$, the three components of the electric and magnetic fields that are present [Mahmoud, 2010] [Dudley et al., 2007]. Any electric field component $E(x, y, z)$ can be expressed as a sum of the modal components [Molina-Garcia-Pardo et al., 2011]:

$$
E(x, y, z)=\sum_{m} \sum_{n} A_{m n}(0) e_{m n}(x, y) \mathrm{e}^{-\gamma_{m n} z}
$$

where $A_{m n}(0)$ is the complex amplitude of the mode in the excitation plane, $e_{m n}(x, y)$ is the normalized modal eigenfunction, and $\gamma_{m n}$ is the complex propagation constant, often written as $\gamma_{m n}=\alpha_{m n}+j \beta_{m n}$. 
It is interesting to introduce the weight of the modes $A_{m n}(z)$ at any abscissa $z$ by stating:

$$
E(x, y, z)=\sum_{m} \sum_{n} A_{m n}(z) e_{m n}(x, y)
$$

where $A_{m n}(z)=A_{m n}(0) \mathrm{e}^{-\gamma_{m n} z}$.

The analytical expressions of the modal eigenfunctions are usually obtained by writing the boundary conditions on the internal surface of the guiding structure. However, in the case of lossy dielectric walls, as in the case of a tunnel, approximations are needed and they are detailed in [Emslie et al., 1975] [Laakmann and Steier, 1976]. It has been previously outlined that rays remain polarized if the distance between Tx and Rx is larger than a few times the transverse dimensions of the tunnel. In the modal theory, the same kind of approximation is performed. If the tunnel is excited by a vertical (y-directed) dipole, the hybrid modes $E H_{m n}^{y}$ are such that the vertical electric field is dominant. For an $x$-directed dipole, the modes are denoted $E H_{m n}^{x}$. The expressions of the modal functions $e_{m n}^{V}(x, y)$ for the $y$-polarized modes, and $e_{m n}^{H}(x, y)$ for the $x$-polarized modes can be found in [Mahmoud, 2010] [Dudley et al., 2007]. The solution of the modal equation leads to expressions for the phase and attenuation constants:

$$
\alpha_{m n}=\frac{2}{a}\left(\frac{m \lambda}{2 a}\right)^{2} \operatorname{Re}\left[\frac{1}{\sqrt{\varepsilon_{r}^{*}-1}}\right]+\frac{2}{b}\left(\frac{n \lambda}{2 b}\right)^{2} \operatorname{Re}\left[\frac{\varepsilon_{r}^{*}}{\sqrt{\varepsilon_{r}^{*}-1}}\right]
$$

and

$$
\beta_{m n}=\frac{2 \pi}{\lambda}\left[1-\frac{1}{2}\left(\frac{m \lambda}{2 a}\right)^{2}-\frac{1}{2}\left(\frac{n \lambda}{2 b}\right)^{2}\right]
$$

Equations (4.4) and (4.5) shows that the attenuation is inversely proportional to the waveguide dimension cubed and the frequency squared. It must be stressed that, given the finite conductivity of the tunnel walls, the modes are not precisely orthogonal. Nevertheless, numerical applications indicate that, when considering the first 60 modes with orders $m=11$ and $n=7$, the modes can be considered as practically orthogonal [Lienard et al., 2006] [Molina-Garcia-Pardo et al., 2008a].

For a vertical transmitting elementary dipole situated at $\left(x_{t x}, y_{t x}\right)$, the total electric field at the receiving point $(x, y, z)$ can be just determined from [Molina-Garcia-Pardo et al., 2008b]:

$$
E(x, y, z)=\sum_{m} \sum_{n} e_{m n}^{V}\left(x_{t x}, y_{t x}\right) e_{m n}^{V}(x, y) \mathrm{e}^{-\gamma_{m n} z}
$$




\subsubsection{Diversity Techniques}

Some diversity techniques are reviewed in the following sections. These diversity techniques are based in the frequency and polarization domain in Section 4.1.2.1, and Section 4.1.2.2 respectively. Time Reversal, that can be considered as a kind of delay-space diversity technique is considered in Section 4.1.2.3.

\subsubsection{Frequency Diversity}

Frequency dimension can be exploited to improve the reliability of the communication or the transmitted data rate. Some WLAN (Wireless Local Area Network) standards such as IEEE 802.11 consider frequency hopping as a frequency diversity scheme. Nevertheless the efficiency of such a technique is strongly dependent on the channel characteristics.

Recently, Orthogonal Frequency Division Mutiplexing (OFDM) has been considered in the latest WLAN (Wireless Local Area Networks) standard such as 802.11n, and in high rate UWB 805.13.3a. One of the main reasons is that OFDM overcomes the drawback of frequency selectivity of the channel by dividing the total bandwidth in $N_{f}$ narrow sub bands in which the channel can be considered to be flat provided that the bandwidth of each sub band is smaller than the channel coherence bandwidth $B_{c}$ (see Appendix B, Section B.5.3). Therefore, its main advantage relies on the simplicity of its one-tap equalization technique and its robustness against inter symbol interference.

OFDM associated with adaptive modulation schemes also offers the advantage of increasing the data rate if the channel is sufficiently "selective" in the transmitted bandwidth. The ideal case would be to get $N_{f}$ independent subchannels. A new metric, called frequency diversity degree, $D_{f}$, can be introduced to quantify the number of independent sub channels. It is defined as the ratio between the transmitted bandwidth and the coherence bandwidth. $D_{f}$ can be considered as the minimum number of sub channels which satisfy the flat fading channel condition.

In a rich scatter environment as in urban area, the advantage of using OFDM has been widely demonstrated. However, in a guiding structure such as tunnels, no results have been found in scientific literature.

\subsubsection{Polarization Diversity}

In tunnels, only waves reflecting on the walls with a grazing angle of incidence, contribute significantly to the received power at large distance from the transmitter, and thus the angular spread of the rays, either at the receiving site or at the transmitting site, is small [Molina-Garcia-Pardo et al., 2003]. 
Since low correlation between array elements is an important criterion for MIMO configurations, this can lead, in a tunnel, to arrays whose length may become prohibitive, due to implementation constraints which have to be fulfilled.

To improve the performance of the link and/or to decrease the size of the array, polarization diversity has been proposed as a solution to reduce the equipment size of MIMO terminals. By using orthogonally-polarized, co-located antennas, it is possible to reduce the interantenna correlation while keeping a compact equipment size [Erceg et al., 2004] [Zhao et al., 2003] [Kyritsi and Cox, 2002] [Quitin et al., 2009a].

In tunnels, the idea that different electric field polarizations use different propagation channels can be interpreted in terms of modes. Indeed, from the modal expansion of the electric field [Kyritsi and Cox, 2002] [Molina-Garcia-Pardo et al., 2008b], it can be easily shown that each mode received with a reasonably high Signal-to-Noise Ratio (SNR) can be associated to a spatial channel. Since the excited modes depend not only on the position but also on the orientation of each Tx element, polarization diversity allows exciting a larger number of modes. This is beneficial in terms of diversity order and low correlation between array elements. However, the efficiency of such techniques is strongly dependent on propagation characteristics.

\subsubsection{Time-Reversal Technique}

In the case of transmission in a rich scattering environment, Time Reversal (TR) signal processing, usually applied to acoustics [Fink et al., 2000], was recently extended to electromagnetic waves [Lerosey et al., 2004] [Lerosey et al., 2005]. One of the main objectives is to focus the energy on the intended user while minimizing its interference to other users. It may be of great interest for multi-user communication and indoor environments containing multiple wireless communication nodes Barton et al., 2007]. A specific application using TR to enhance data communication in ventilation ducts, thus in a guided structure, is reported in [Henty, 2007].

The idea of combining UWB and TR to take benefit from the multipath propagation in indoor channels has been proposed [Pajusco and Pagani, 2009] [Naqvi et al., 2010]. Another example of performances of TR UWB is given in [Liu et al., 2008], based on typical IEEE Ultra-Wideband (UWB) channel model parameters. A theoretical study of TR-UWB for train-to-wayside communication, and based on a software tool simulating the propagation in a rectangular tunnel was presented in [Saghir et al., 2009a], while modified-orthogonal waveforms for ensuring multiple access communications are discussed in [Saghir et al., 2009b].

The main characteristic of Time Reversal is the space and time compression. The channel impulse response, $h(\tau)$ is estimated. Next, this response is inverted and conjugated, being $h^{*}(\tau)$. This new channel impulse response is used as the pre-coding of the transmitted UWB pulse. Therefore, 
let $x(t)$ be the transmitted signal, the received signal $y(t)$ can be expressed as:

$$
y(\tau)=\left(x(\tau) \otimes h^{*}(-\tau)\right) \otimes h(\tau)=x(\tau) \otimes\left(h(\tau) \otimes h^{*}(-\tau)\right)=x(\tau) \otimes h_{e q}(\tau)
$$

where $h_{e q}(\tau)$ is the equivalent channel impulse response using time reversal, which is defined as:

$$
h_{e q}(\tau)=h^{*}(-\tau) \otimes h(\tau)
$$

This expression is the autocorrelation of the channel impulse response. It should be also considered normalize the equivalent channel impulse response in order to fairly compare this technique with other schemes [Pajusco and Pagani, 2009];

$$
h_{e q}(\tau)=\frac{h^{*}(-\tau)}{\sqrt{\int\left|h^{*}(-\tau)\right|^{2}}} \otimes h(\tau)
$$

In order to evaluate the features of Time Reversal, some figures of merit are proposed in literature. In [Pajusco and Pagani, 2009] the authors propose the study of the gain depending on the kind of receiver used: those which only detects the main peak of the received signal or those which acquires all the energy of the channel impulse response. If the receiver only detects the main peak of the received signal, the peak to peak gain is defined as follows:

$$
G_{p 2 p}=10 \log _{10}\left(\frac{\max \left(\left|h_{e q}(\tau)\right|^{2}\right)}{\max \left(|h(\tau)|^{2}\right)}\right)
$$

However, if the receiver captures all the energy of the channel impulse response, the total power gain is defined as:

$$
G_{\text {pow }}=10 \log _{10}\left(\frac{\int\left|h_{e q}(\tau)\right|^{2}}{\int|h(\tau)|^{2}}\right)
$$

As mentioned before, spatial compression is one of the most important characteristics of Time Reversal. Multi-user systems profit from this quality as it is studied in [Nguyen et al., 2006]. As it is shown in Figure 4.2, the equivalent channel impulse response for TX-RX2 is not optimal because of the use of the estimated channel impulse response of TX-RX1. For this reason, it can be assured that this is an advantageous technique because the signal can be optimally received by the target receiver while it produces a kind of error in the non-target receivers.

However, this technique presents the disadvantage that if the channel varies between the estimation and the transmission of the coded signal, it will suffer from error due to the bad estimation of the channel. This is the reason why this technique is strongly recommended in slow fading channels. 


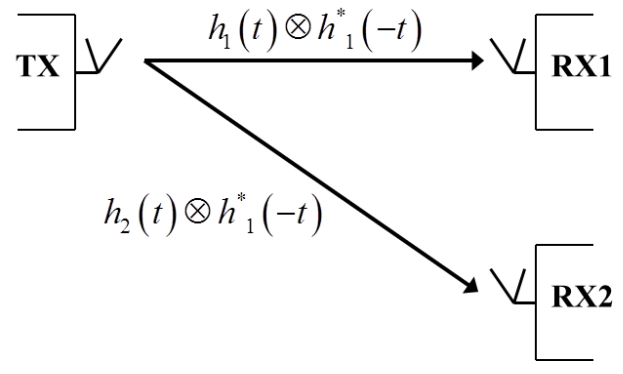

Figure 4.2. Situation in case of several receivers.

\subsection{Description of the Measurement Campaign}

The straight tunnel wherein our measurements were performed is shown in Figure 4.3. This 3 $\mathrm{km}$-long tunnel was closed to traffic during the experiments so the propagation channel was therefore stationary. The transverse section of the tunnel was semicircular and the diameter of the cylindrical part was $8.6 \mathrm{~m}$. The maximum height was $6.1 \mathrm{~m}$ at the center of the tunnel. The roughness of the walls is quite low, in the order of less than $2 \mathrm{~cm}$.

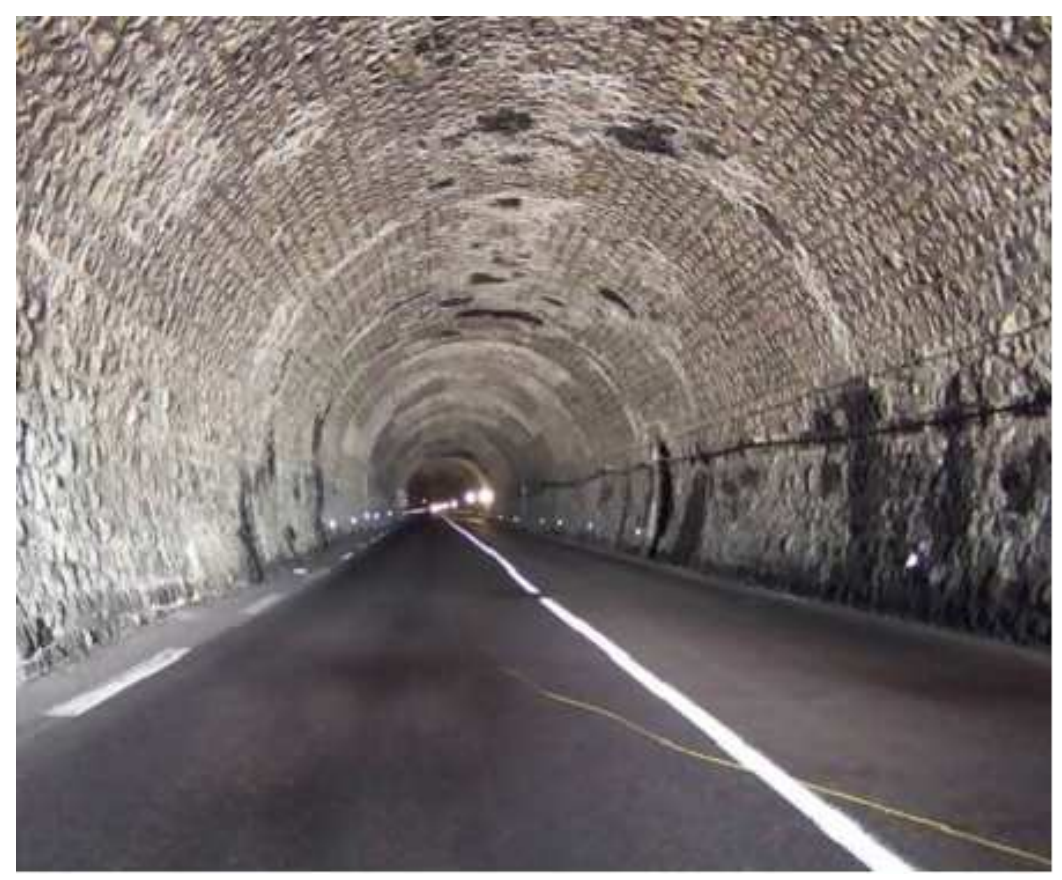

Figure 4.3. Photo of the tunnel where measurements were carried out.

\subsubsection{Measurement Equipment}

The complex channel transfer function between the transmitting and receiving antennas has thus been obtained by measuring the $S_{21}$ parameter with a vector network analyzer VNA Agilent E5071B). Using a coaxial cable to connect the Tx antenna to one port of the VNA would lead to prohibitive attenuation, the maximum distance between Tx and Rx being $500 \mathrm{~m}$. 
The signal of the Tx port of the VNA is thus connected to a distributed feedback laser transmitter (ORTEL 3541A) working in a frequency range extending from $100 \mathrm{MHz}$ to $10 \mathrm{GHz}$, the amplitude flatness in this whole band being $\pm 2.5 \mathrm{~dB}$. The optical signal is sent through a monomode fibre optics presenting a very low attenuation $(2 \mathrm{~dB} / \mathrm{km})$. It is then converted back to radio frequency owing to a wideband photodiode receiver (Agere 4518B), whose amplitude flatness is the same as for the transmitter. In our frequency band of interest $(2.8-5 \mathrm{GHz})$, the total loss of the optical chain is $26 \mathrm{~dB} \pm 0.5 \mathrm{~dB}$. The signal is thus amplified so that the output RF signal reaches a transmitter power of $20 \mathrm{dBm}$.

The Rx antenna is directly connected to the other port of the VNA using a low attenuation coaxial cable, $4 \mathrm{~m}$ long, a $30 \mathrm{~dB}$ low-noise amplifier (Nextec NBL00416) having a noise figure of $2.5 \mathrm{~dB}$.

The phase stability of the fibre optics link has been checked and the calibration of the VNA takes into account amplifiers, cables and optic coupler. The block diagram of the channel sounder is depicted in Figure 4.4

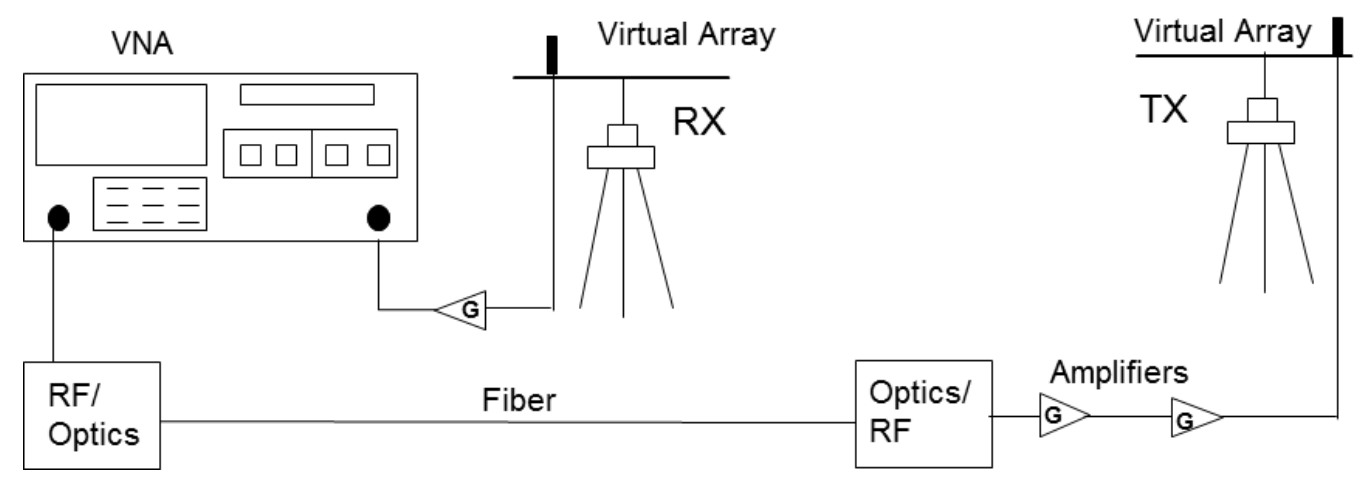

Figure 4.4. Scheme of the channel sounder set up.

The wideband biconical antennas (Electrometrics EM-6116) used in this experiment have nearly a flat gain, between 2 and $10 \mathrm{GHz}$. Indeed, the frequency response of the two antennas has been measured in an anechoic chamber, and the variation of the antenna gain was found to be less than 2 $\mathrm{dB}$ in our frequency range. The radiation pattern of wideband antennas is thus also slightly frequency dependent. This is not a critical point in our case since, in a tunnel, only waves impinging the tunnel walls with a grazing angle of incidence contribute to the total received power significantly. This means that, whatever the frequency, the angular spread of the received rays remains much smaller than the $3 \mathrm{~dB}$ beamwidth of the main antenna lobe in the $\mathrm{E}$ plane, equal to about $80^{\circ}$, the antenna being nearly omnidirectional in the H plane.

Virtual arrays were obtained by moving the Tx and Rx antennas along a rail. The position mechanical systems are remotely controlled, with a precision of $\pm 0.5 \mathrm{~mm}$, optic fibres connecting the step by step motors to the control unit situated near the VNA. The battery-driven mobile equipment was mounted on a wooden rolling table which was manually pulled. 


\subsubsection{Methodology}

The channel frequency response has been measured for 1601 frequency points, equally spaced between 2.8 and $5 \mathrm{GHz}$, leading to a frequency step of $1.375 \mathrm{MHz}$.

The rails supporting the Tx and Rx antennas were put at a height of $1 \mathrm{~m}$ and centred on the same lane of this 2-lane tunnel, thus at $1 / 4$ of the tunnel width. For each successive axial distance $d$, both the Tx and Rx antennas were moved in the transverse plane on a distance of $33 \mathrm{~cm}$, with a spatial step of $3 \mathrm{~cm}$, corresponding to half a wavelength at $5 \mathrm{GHz}$. A $12 \times 12$ transfer matrix is thus obtained, the configuration of the measurements being schematically described in Figure 4.5. Fine spatial sampling was chosen for measurements in the transverse plane to be able to extract information of the direction of departure/arrival of the rays.

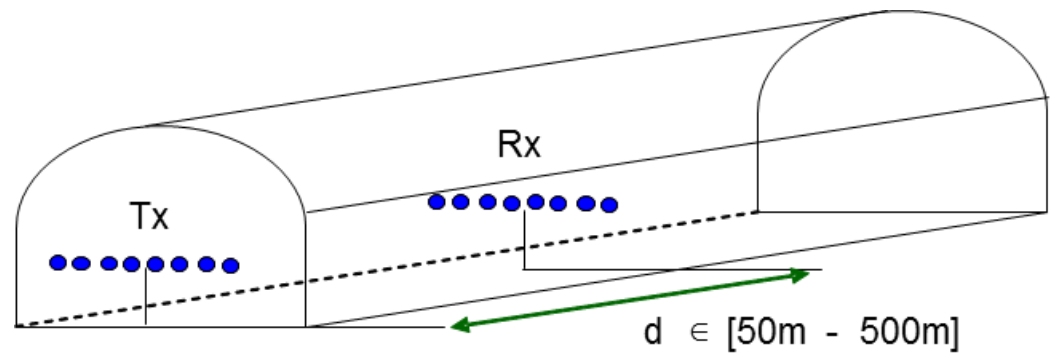

Figure 4.5. Configuration of the wideband measurements.

Due to the limited time available for such an experiment and to operational constraints, it was not possible to extensively repeat such measurements for very small steps along the tunnel axis. In the experiments described in this thesis, the axial step was chosen equal to $4 \mathrm{~m}$ when $50 \mathrm{~m}<d<202$ $\mathrm{m}$ and to $6 \mathrm{~m}$ when $202 \mathrm{~m}<d<500 \mathrm{~m}$.

Measurements are made in successive static conditions. At each Tx and Rx position, 5 successive recordings of field variation versus frequency are stored and averaged. A summary of the measurement parameters and equipment characteristics is summarized in Table 4.1.

\begin{tabular}{||c|c||}
\hline \hline Frequency Band & $2.8-5 \mathrm{GHz}$ \\
\hline Number of Frequency Points & 1601 \\
\hline Antenna & Biconical antenna (Electrometrics EM-6116) \\
\hline Transmitter Power & $20 \mathrm{dBm}$ \\
\hline Dynamic Range & $>100 \mathrm{~dB}$ \\
\hline Positions in the Transverse Plane & 12 positions every $3 \mathrm{~cm}(\lambda / 2$ at $5 \mathrm{GHz})$ \\
\hline Positions along the Longitudinal Axis & From $50 \mathrm{~m}$ to $202 \mathrm{~m}$ every $4 \mathrm{~m}$ \\
& From $202 \mathrm{~m}$ to $500 \mathrm{~m}$ every $6 \mathrm{~m}$ \\
\hline Number of Acquisitions at each Position & $5 \mathrm{~dB}$ \\
\hline \hline
\end{tabular}

Table 4.1. Equipment characteristics and measurement parameters. 


\subsubsection{Polarization}

In the same measurement campaign several polarizations of the transmitting and receiving arrays where taken into account. In order to avoid an exhaustive parametric study, only $4 \times 4 \mathrm{MIMO}$ links are studied by assuming a fixed total length of the virtual uniform linear array equal to $18 \mathrm{~cm}$, the inter element spacing being $6 \mathrm{~cm}$. In this case, the following combinations of polarization were:

- VV, where all Tx and Rx elements are vertically polarized.

- HH, where all Tx and Rx elements are vertically polarized

- VH, where all elements are vertically polarized while all Rx elements are horizontally polarized.

- VHVH, where both the first and the third elements of each array are vertically polarized, while the second and the fourth elements are horizontally polarized.

- Dual, where Tx or Rx elements are dual-polarized. For this special configuration, the spacing between the two dual-polarized elements is $18 \mathrm{~cm}$, i.e. the length of the previous arrays.

For each polarization and each combination of transmitting and receiving antennas $(4 \times 4=16$ in total), the transfer matrix $G(d, f)$ can be deduced from measurements, where $d$ is the axial distance and $f$ the frequency.

\subsection{Experimental UWB Channel Characterization}

This section deals with the experimental characterization of the radio channel in tunnels. In the follwing sections a detailed analysis of classical parameters such as the mean path loss, the RMS delay spread, the power delay profile, the complex correlation coefficient, or the channel coherence bandwidth are deduced.

The knowledge of such parameters is an essential issue in order to evaluate the performance in tunnel of the diversity techniques proposed in Section 4.1.2 and that will be evaluated in Section 4.4.

\subsubsection{Mean Path Loss and Small Scale Fading}

In narrow band communication systems, path loss is frequency and distance dependent, while small scale fading is due to the constructive and destructive interference of all possible paths between Tx and Rx. For Impulse Radio (IR) UWB (measurements bandwidth $2.8-5 \mathrm{GHz}$ ) signals, the most important characteristics are related to the variation in the energy of the pulse and not of its individual spectral components. Figure 4.6 gives the value of the UWB measured path loss defined 
as the ratio of the Tx power to the received power at a distance $d$, this ratio being averaged over the whole frequency band and over the $12 \times 12$ transverse positions of Tx and Rx. I can be observed the guiding effect of the tunnel, the additional attenuation between $100 \mathrm{~m}$ and $500 \mathrm{~m}$ only being $4 \mathrm{~dB}$. The variation of experimental values of path loss can be modeled by a decay factor of 0.55 , as shown in Figure 4.6

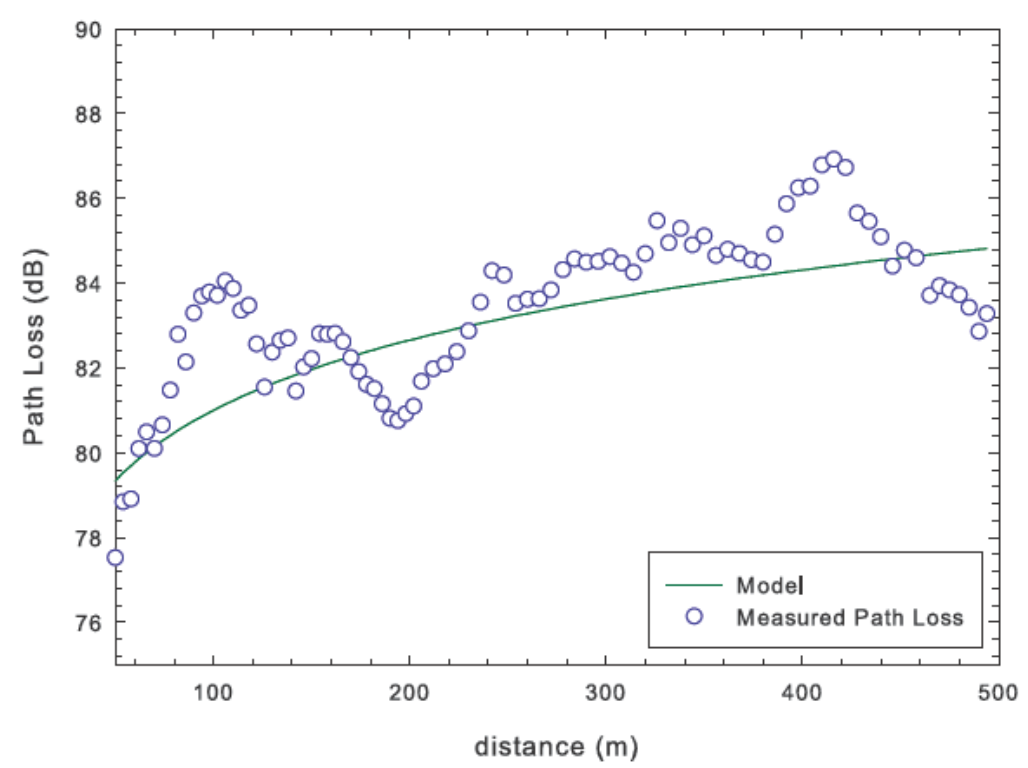

Figure 4.6. Mean path loss versus distance.

To determine the characteristics of the small-scale fading, a rectangular grid containing successive Rx positions is considered. The width of the grid, i.e. its dimension along the transverse axis, is equal to $33 \mathrm{~cm}$ and corresponds to the maximum displacement of the antenna in the transverse plane. Along the tunnel axis, 3 successive distances as shown in Figure 4.7 are taken into account.

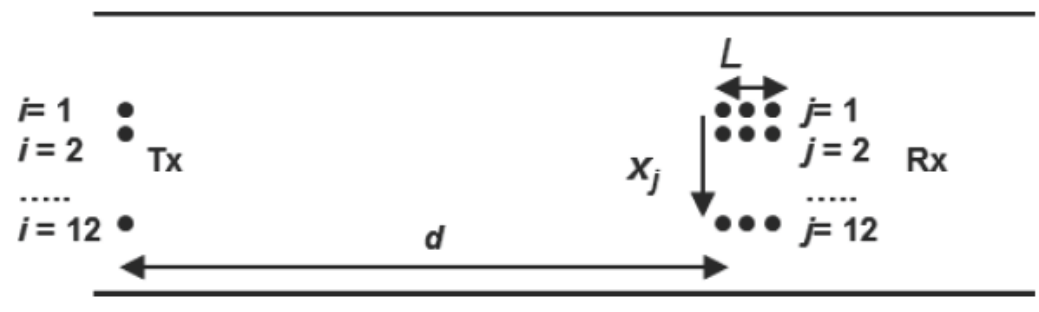

Figure 4.7. Successive positions of $\mathrm{Rx}$ for calculating the standard deviation of the path loss. $L=8 \mathrm{~m}$ for $d \leq 200 \mathrm{~m}$ and $L=12 \mathrm{~m}$ for $206 \mathrm{~m} \leq d \leq 486 \mathrm{~m}$.

Thus the length $L$ of the grid is either $8 \mathrm{~m}$ or $12 \mathrm{~m}$ depending on whether the distance $d$ between Tx and Rx is smaller or larger than $206 \mathrm{~m}$, as explained in Section 4.2. The standard deviation $(s t d)$ of the path loss in the rectangular grid, normalized to its average value in this grid and thus expressed in \%, was calculated for successive values of $d$ varying between $50 \mathrm{~m}$ and $486 \mathrm{~m}$ and also by considering, for each value of $d$, the 12 possible positions of the Tx antenna in the transverse plane of 
the tunnel. It appears that std varies between $8 \%$ and $20 \%$, depending on the axial distance between Tx and Rx. This means that there is almost no fading as a result of interference and this is the same conclusion as in the case of propagation in a typical in-building environment [Muqaibel et al., 2006] and [Molisch, 2009].

The analysis of the delay dispersion is also analyzed in Section 4.4.3.2 for examining the role of the Time Reversal technique on the reduction of $D_{s}$.

\subsubsection{Channel Characterization in Space-Delay-Frequency Domains}

In this section, the results from the wideband analysis of the radio channel are presented. First, the complex correlation coefficient is evaluated in Section 4.3.2.1 Next, the power delay profile and the delay dispersion are presented in Section 4.3.2.2. The double directional parameters of the channel are deduced from measurements by using the RIMAX algorithm in Section 4.3.2.3. Finally, in Section 4.3.2.4 the coherence bandwidth is deduced to provide information about flatness of the channel in frequency.

\subsubsection{Correlation}

In this section, the complex correlation coefficient is evaluated. First, the transverse correlation at the transmitter is analyzed as a function of distance Tx-Rx along the tunnel. Next, the axial correlation between two consecutive positions of the transmitter is also considered.

\section{A. Transverse Correlation}

The complex correlation coefficient, $\rho$, at the transmitter at the initial and final frequency of the measured frequency band (2.8 and $5 \mathrm{GHz}$ respectively) has been computed (see also Appendix $\mathrm{A}$ and Section A.3 and results are shown in Figure 4.8.

For $f=2.8 \mathrm{GHz}$, the channel is uncorrelated $(\rho<0.7)$ only at the beginning of the tunnel, along the first 100 meters. This effect is more noticeable for a larger antenna spacing $(12 \mathrm{~cm})$ as expected. The same behavior is observed at $f=5 \mathrm{GHz}$, although the transverse correlation increases considerably after the first 150 meters. Again, the higher the antenna spacing is, the lower the transverse correlation is.

The effect of a higher antenna spacing on the decrease in the transverse correlation is clearly observed in Figure 4.9 where the complex correlation coefficient has been averaged over all frequencies. From this figure it can be deduced that for an antenna spacing of $6 \mathrm{~cm}$ the channel is uncorrelated only in the first 100 meters. Nevertheless, if the antenna spacing is doubled, the correlation distance increases up to nearly 150 meters. 


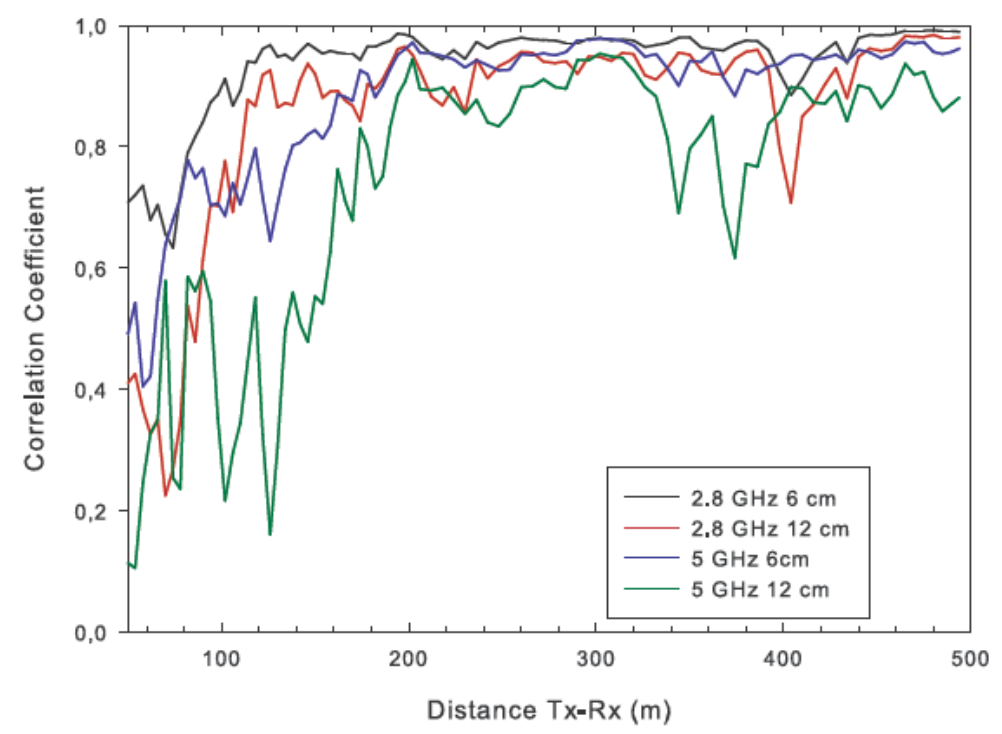

Figure 4.8. Transverse correlation for $f=2.8 \mathrm{GHz}$ and $f=5 \mathrm{GHz}$ for different antenna spacings, $6 \mathrm{~cm}$ and $12 \mathrm{~cm}$.

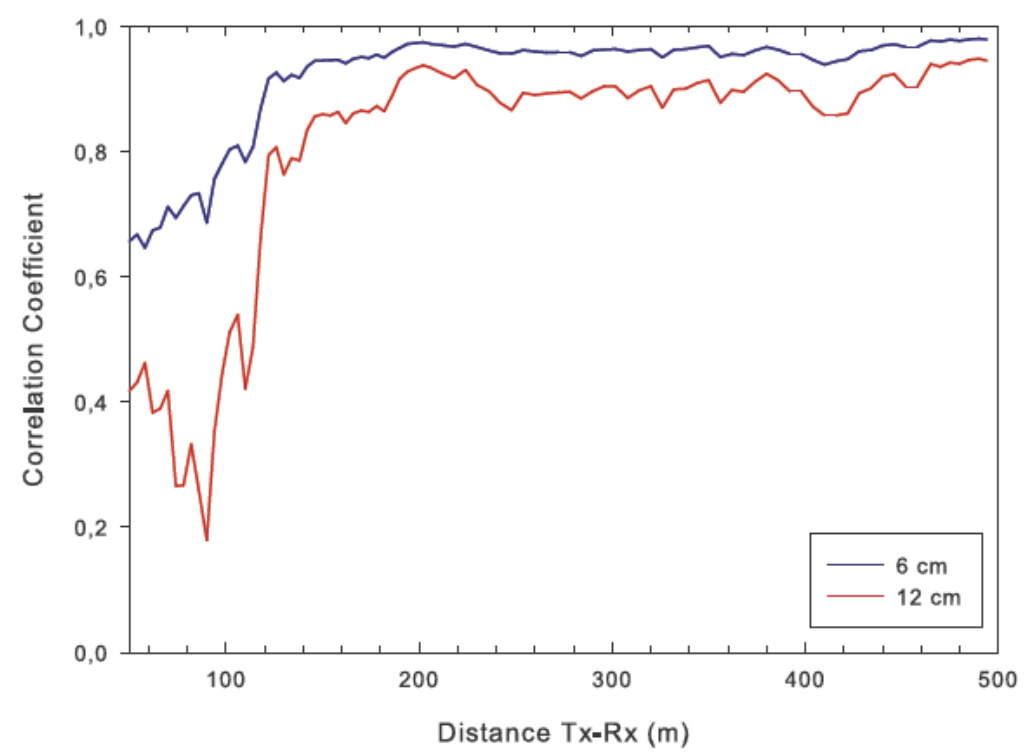

Figure 4.9. Mean transverse correlation for different antenna spacings, $6 \mathrm{~cm}$ and $12 \mathrm{~cm}$.

\section{B. Axial Correlation}

Due to experimental constraints, the spacing between successive measurement points along the tunnel axis were $d=4 \mathrm{~m}$ or $6 \mathrm{~m}$, depending on the distance $d$. This axial step $d$ is thus not small enough to clearly point out the continuous decrease of the correlation coefficient versus the antenna spacing. 
However, it is interesting to point out the variation of the average complex correlation between receiving points situated at $d$ and $d+\Delta d$ (with $\Delta d$ the distance between two consecutive points along the tunnel), when $d$ varies from $50 \mathrm{~m}$ to $498 \mathrm{~m}$. This average complex correlation is defined, as for the transverse correlation, by:

$$
\rho(d, d+\Delta d)=<\rho(i, d, d+\Delta d)>_{i}
$$

where $i$ denotes the position of a Tx antenna. The results plotted in Figure 4.10 show that in the first zone, i.e. when $d$ varies from $50 \mathrm{~m}$ to $206 \mathrm{~m}$ and $\Delta d=4 \mathrm{~m}$, the correlation coefficient varies from 0.4 to 0.9 .

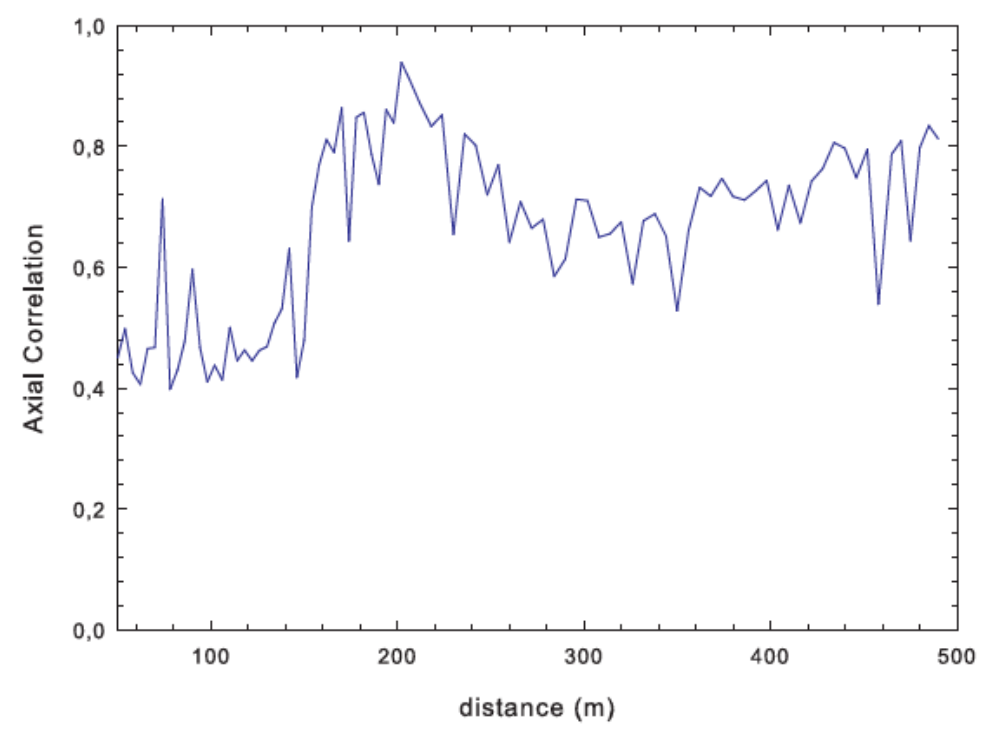

Figure 4.10. Axial correlation coefficient versus distance $d$ between 2 points, $4 \mathrm{~m}$ apart (if $d<204$ $\mathrm{m}$ ) or $6 \mathrm{~m}$ apart (if $d>204 \mathrm{~m}$ ).

The high value of the correlation coefficient around $d=200 \mathrm{~m}$ can be explained by a strong attenuation of the multipath components as previously outlined. In the second zone of measurement, i.e. when $250 \mathrm{~m}<d<498 \mathrm{~m}$ and $\Delta d=6 \mathrm{~m}$, the correlation increases from 0.7 to 0.8 . The average increase of the correlation coefficient between $50 \mathrm{~m}$ and $500 \mathrm{~m}$, can be explained, as in the case of the correlation in the transverse plane, by the decrease of the number of modes propagating at large distances from the transmitter.

\subsubsection{Power Delay Profile and Delay Spread}

As the channel was stationary during measurements, the channel transfer function in the frequency domain $H(d, i, j, f)$ is measured with the experimental set up described in Section 4.2 The variables $i$ and $j$, varying from 1 to 12 , correspond to the various locations of the antennas in the transverse plane. 
Thus, the complex channel impulse response at a distance $d$ between $T x$ and $R x$, expressed as $h(d, i, j, \tau)$, where $\tau$ represents the channel multipath delay, is then obtained by applying a Hamming window and an inverse Fourier transform to $H(d, i, j, f)$.

\section{A. Power Delay Profile}

The power delay profile, is found by taking the spatial average of $|h(d, i, j, \tau)|^{2}$ [Richter and Thoma, 2005]. In the following examples, the axial distance (along the tunnel) $d$ is larger than $50 \mathrm{~m}$, whereas in the transverse plane, the antennas move along a maximum distance of $33 \mathrm{~cm}$. Thus, for a given value of $d$, the propagation delay due to the difference in distances between the successive transverse elements of $\mathrm{Tx}$ and $\mathrm{Rx}$ is smaller than $0.03 \mathrm{~ns}$ which is quite negligible. Consequently, the $\operatorname{PDP}(d, \tau)$ can be obtained by simply averaging $|h(d, i, j, \tau)|^{2}$ over the $12 \times 12$ positions $(i, j)$ of the antennas. At each distance $d$, the power of the strongest path of the Power Delay Profile (PDP) is then normalized to $0 \mathrm{~dB}$. All successive normalized PDPs at different distances have been gathered in Figure 4.11. It must be emphasized that the delays presented are always measured relative to free space delay, corresponding to the direct path. Only paths whose amplitudes are attenuated less than $20 \mathrm{~dB}$ are represented in Figure 4.11 and in the following figures. The continuous decrease in the delays between successive rays, at least for distances smaller than $200 \mathrm{~m}$ can also be observed.

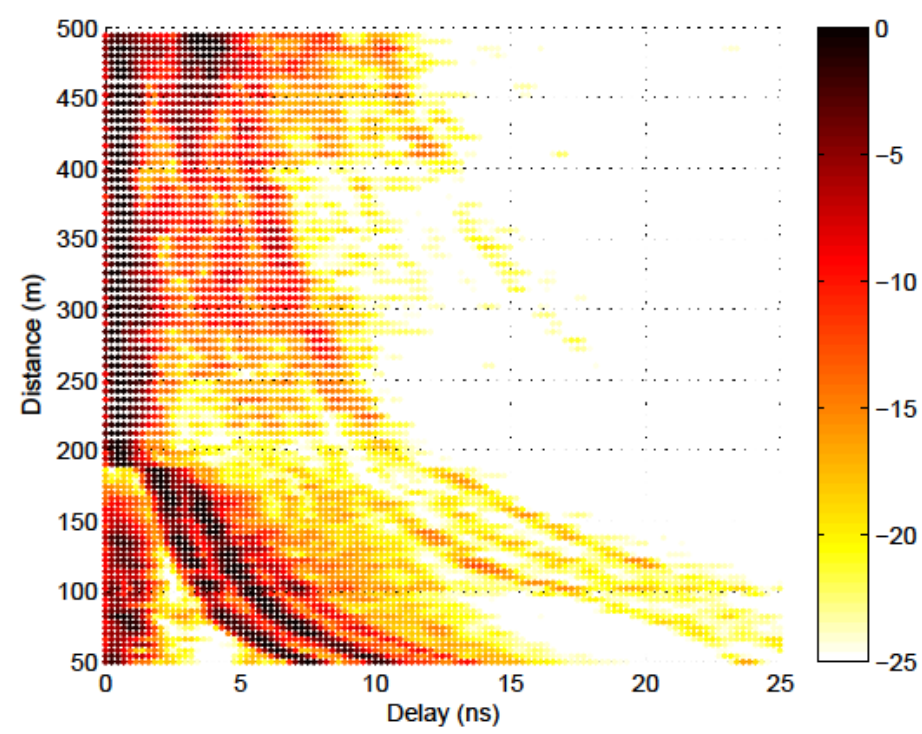

Figure 4.11. PDPs deduced from measurements and normalized to $0 \mathrm{~dB}$ corresponding to their maximum power.

It is interesting to compare these experimental results with theoretical values. However, the simulation of the propagation in an arched tunnel over a large distance and over a wide frequency band is not an easy task and, and thus a very simple model based on the image theory has been 
chosen. Indeed, it was shown in [Molina-Garcia-Pardo et al., 2008b] that for frequencies of $500 \mathrm{MHz}$ and $900 \mathrm{MHz}$ it was possible to interpret both path loss and fading in this arched tunnel by means of an equivalent straight rectangular tunnel, $8 \mathrm{~m}$ wide and $5.6 \mathrm{~m}$ high, with walls of an equivalent conductivity and permittivity equal to $10^{-2} \mathrm{~S} / \mathrm{m}$ and 5 , respectively. Using these values, and with the same bandwidth as in the experiments $(2.8-5 \mathrm{GHz})$, the PDPs deduced from the propagation model are given in Figure 4.12

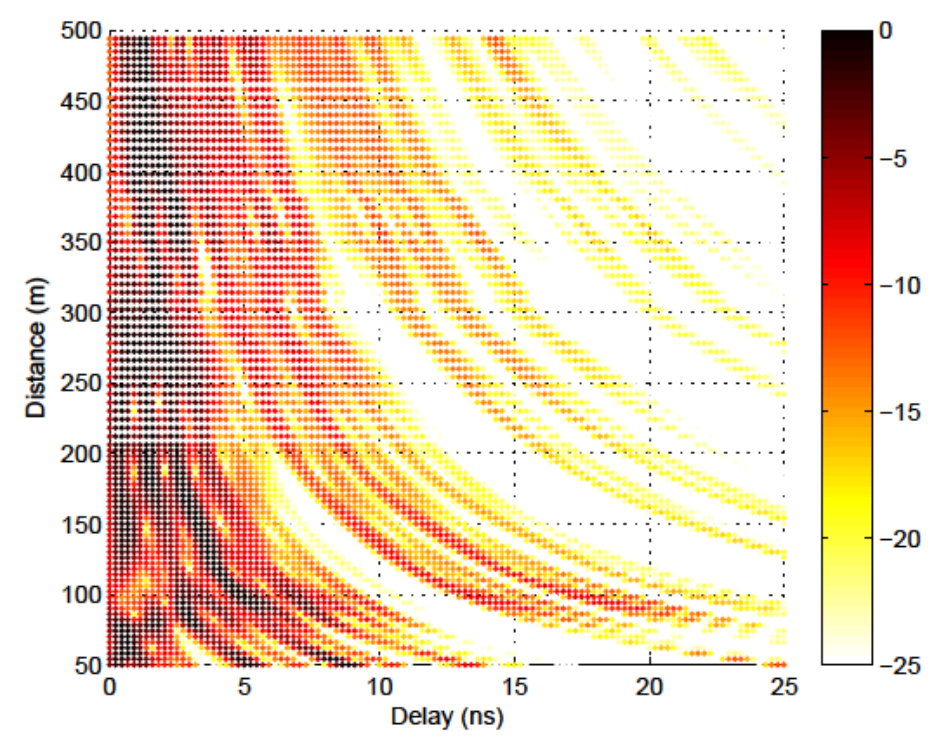

Figure 4.12. PDPs obtained from a theoretical propagation model, assuming a tunnel of rectangular cross section $(8 \times 5.6 \mathrm{~m})$ and the same frequency bandwidth as in the measurements.

At a short distance from $\mathrm{Tx}$, between $50 \mathrm{~m}$ and $100 \mathrm{~m}$, the successive paths can be clearly distinguished in both figures. At $50 \mathrm{~m}$, the maximum delay, considering a relative attenuation of $10 \mathrm{~dB}$ referred to the strongest path, is $10-15 \mathrm{~ns}$. When this distance increases, only rays impinging the tunnel wall with a grazing angle of incidence play a leading part, and this gives rise to the typical shape of the plots drawn in Figure 4.11 and Figure 4.12 In these figures, local decreases of the received power also appear. As an example, Figure 4.12 shows that the signals arriving during the first $3 \mathrm{~ns}$ are attenuated in a zone situated at about $100 \mathrm{~m}$ from Tx. Indeed, even with a $2.2 \mathrm{GHz}$ bandwidth, the time resolution is not high enough to separate the contribution of individual rays and destructive (or constructive) interference may occur. The ray tracing model, assuming an infinite bandwidth and a rectangular tunnel, shows that 32 rays, having a relative attenuation of less than 10 $\mathrm{dB}$, arrive during a time window of $10 \mathrm{~ns}$, and 10 rays reach the Rx during the first $3 \mathrm{~ns}$.

\section{B. RMS Delay Spread}

In the delay domain, an interesting parameter characterizing the channel properties is the RMS (Root Mean Square) delay spread, $D_{s}$, defined as the normalized second-order moment of the channel 
impulse response (see Appendix $B$ Section B.5.2). For each axial distance $d$, the delay spread is deduced from the channel impulse response calculated for each of the $12 \times 12$ channels associated with the possible positions of the antennas in the transverse plane.

Since it is interesting to point out the variation of $D_{s}$ both on a small scale and on a large scale, the tunnel was divided into 4 zones. The first one extends from $50 \mathrm{~m}$ to $100 \mathrm{~m}$ and henceforth will be called the "near" zone. Other zones correspond to distance intervals from $100 \mathrm{~m}$ to $200 \mathrm{~m}$, from $200 \mathrm{~m}$ to $300 \mathrm{~m}$, from $300 \mathrm{~m}$ to $400 \mathrm{~m}$ and finally from $400 \mathrm{~m}$ to $500 \mathrm{~m}$. Figure 4.13 shows the complementary cumulative distribution function (CCDF) of the local $D_{s}$, deduced from experimental data.

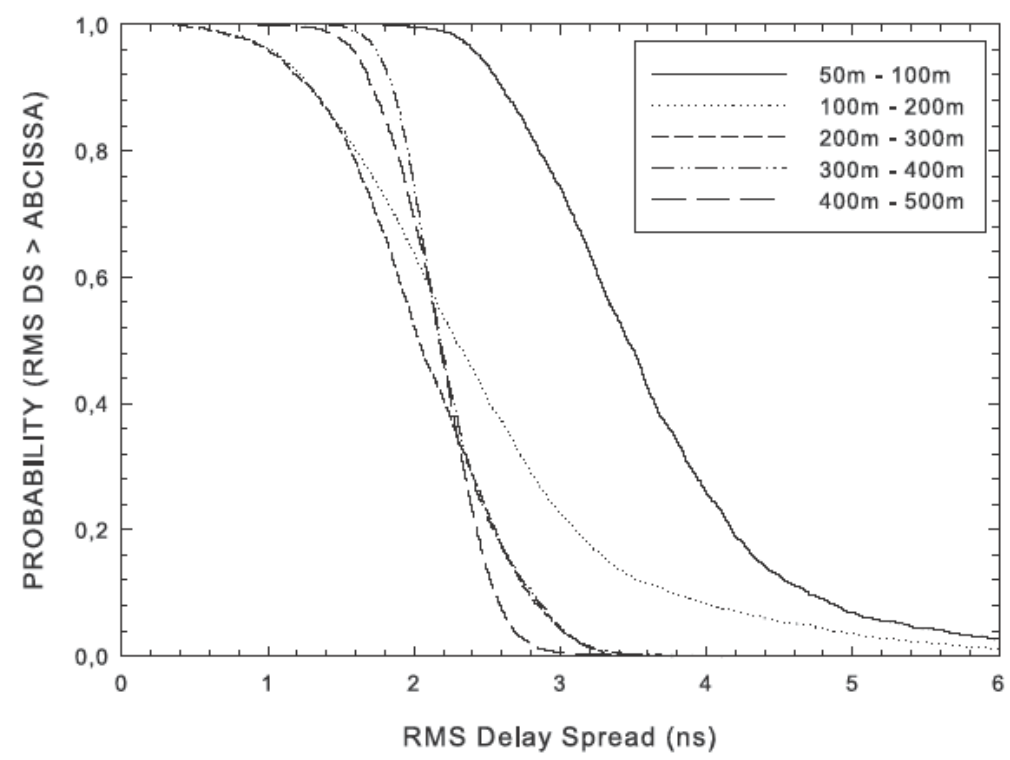

Figure 4.13. CCDFs of the local rms delay spread deduced from experimental data.

Considering a probability of 0.5 , the curves in Figure 4.13 show that the $D_{s}$ is in the order of 3.6 $\mathrm{ns}$ in the near zone $(50 \mathrm{~m}-100 \mathrm{~m})$, but then decreases to $2.5 \mathrm{~ns}$ beyond $100 \mathrm{~m}$ and does not vary very much with distance. This can also be observed by visually interpreting Figure 4.12 For a rectangular tunnel, $8 \times 5.6 \mathrm{~m}$, the theoretical values of the $D_{s}$ deduced from the ray approach are larger and vary from $6 \mathrm{~ns}$ in the near zone to $4 \mathrm{~ns}$ beyond $100 \mathrm{~m}$. The difference may be due to the idealized rectangular shape of the tunnel in the theoretical model, instead of the curved shape of the tunnel where experiments took place. The values of $D_{s}$ can be compared to those measured in an empty tunnel, without obstacles, and described by other authors as in [Boutin et al., 2008 and [Zhang and Hwang, 1998]. In a $70 \mathrm{~m}$ long mine gallery, it was shown [Boutin et al., 2008] that $D_{s}$ in the $2.4 \mathrm{GHz}$ band is less than or equal to $6.3 \mathrm{~ns}$ for $50 \%$ of all locations. The corresponding value for the $5.8 \mathrm{GHz}$ band is $5 \mathrm{~ns}$. Delay spread in a rectangular concrete subway tunnel, $3.5 \mathrm{~m}$ wide and 2.6 $\mathrm{m}$ high, at a carrier frequency of $1.8 \mathrm{GHz}$ and over a $400 \mathrm{MHz}$ band was found to be equal to $5.5 \mathrm{~ns}$ for a $50 \mathrm{~m}$ separation between the transmit and receive antennas [Zhang and Hwang, 1998]. 
In Figure 4.13, one can also note that when the distance increases from $100 \mathrm{~m}$ to $500 \mathrm{~m}$, the slopes of the CCDF curves become steeper corresponding to a decrease of the spread of the $D_{s}$. For example, if a probability interval of $0.1-0.9$ is considered, $D_{s}$ is between 1.3 and 3.9 ns within the $100 \mathrm{~m}-200$ $\mathrm{m}$ range from $\mathrm{Tx}$ and between 1.9 and $2.5 \mathrm{~ns}$ within the $400 \mathrm{~m}-500 \mathrm{~m}$ range from Tx.

\subsubsection{Angular Power Spectrum and Angular Spread}

Multiple replicas of the radio signal from different directions in space give rise to spatial diversity (see Appendix $\mathrm{A}$ ), which can be used to increase the transmission reliability of the fading radio link. Since the higher the spatial correlation, the lower the diversity order is, spatial diversity is therefore an important channel characteristic [Sibille et al., 2011].

The double directional channel describes the propagation channel and separates the effect of transmitting and receiving antennas from the propagation environment as depicted in Figure 4.14 On the one hand, the transmitting antenna distributes the signal energy into the desired Directions of Departure, $\mathrm{DoD}$ (also called Angles of Departure, $\mathrm{AoD}$ ). On the other hand, the receiving antenna collects the signal components from the Directions of Arrival, DoAs (also called Angles of Arrival, AoA by weighted combination. Thus, the double directional channel includes all resolvable propagation paths, between the transmitter and the receiver sites. Each path is delayed in accordance to its excess delay, $\tau$, weighted with the proper complex amplitude, and each $\operatorname{DoD}, \varphi_{T}$, is connected to the corresponding DOA, $\varphi_{T}$ [Steinbauer et al., 2001].

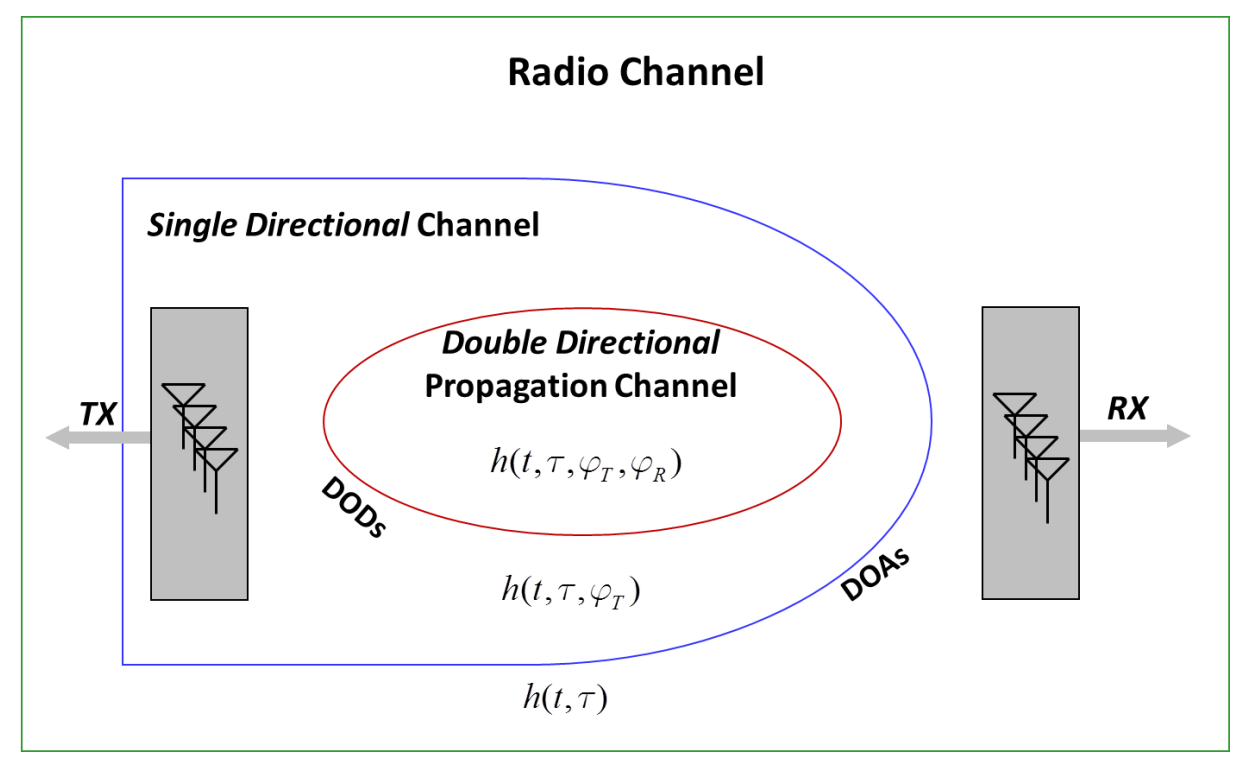

Figure 4.14. Scheme of the double directional channel. 
High resolution parameter estimation algorithms have been widely employed to estimate the parameters of the double directional channels. MUSIC (Multiple Signal Classification) [Schmidt, 1986], ESPRIT (Estimation of Signal Parameters via Rotational Invariance Techniques) [Haardt, 1996], and SAGE (Space Alternating Generalized Expectation maximization) [Fessler and Hero, 1994] are well known algorithms to extract the directions of departure/arrival from experimental measurements. Lately, RIMAX algorithm [Richter, 2005] has emerged as a parameter estimation method which allows joint high-resolution estimation of both the specular propagation paths parameters and of the dense multipath components.

Despite of the characterization of the double directional channel has been widely treat in scientific literature for office environments, there is not too much information about the spatial characterization of the MIMO channel in tunnels.

In this section, the RIMAX algorithm [Richter, 2005] is used to obtain quantitative results about the bidirectional channel characteristics, such as the angle of departure (AoD) and the angle of arrival (AoA) of the paths and the angular spread along the tunnel. This algorithm is a recently developed multidimensional maximum-likelihood high-resolution channel parameters estimator. It is based on the assumption that the radio channel can be modeled as a superposition of a number of finite specular-alike propagation paths and dense multipath components. The algorithm iteratively seeks propagation paths and optimizes their characteristics. It does not require a prior knowledge of the number of dominant propagation paths.

The double directional characteristics of the channel are deduced from measurements and the results are compared with those extracted from theoretical simulations described in Section 4.3.2.2. $A$. During the measurement campaign the virtual antenna arrays were situated on the horizontal plane, the array axis being perpendicular to the tunnel axis. It is thus only possible to compute the azimuth $\varphi$ of arrival or departure of the rays in this plane and defined as the horizontal angular distance from the tunnel axis $z$ to the direction of the rays. A 1D array is not able to distinguish rays propagating along positive or negative values of $z$, i.e. from or towards the transmitter. However, this is not critical for this analysis since in an empty tunnel, no reflection giving rise to a backward propagation may occur.

Firstly, the theoretical azimuth power spectrum APS $(d, \varphi)$ [Molisch, 2011] versus the distance between Tx and Rx, has been calculated from the channel impulse response $h(\tau)$. For each axial distance, the power of the strongest ray, corresponding to the direct path, is normalized to $0 \mathrm{~dB}$. In such a rectangular tunnel, many rays arrive with the same azimuth $\varphi$ and thus only the APS of the most powerful rays are presented in Figure 4.15. Their relative weights in the successive transverse planes (between $50 \mathrm{~m}$ and $500 \mathrm{~m}$ ) are expressed in $\mathrm{dB}$ and are given by the color scale on the right hand side of the figure. 


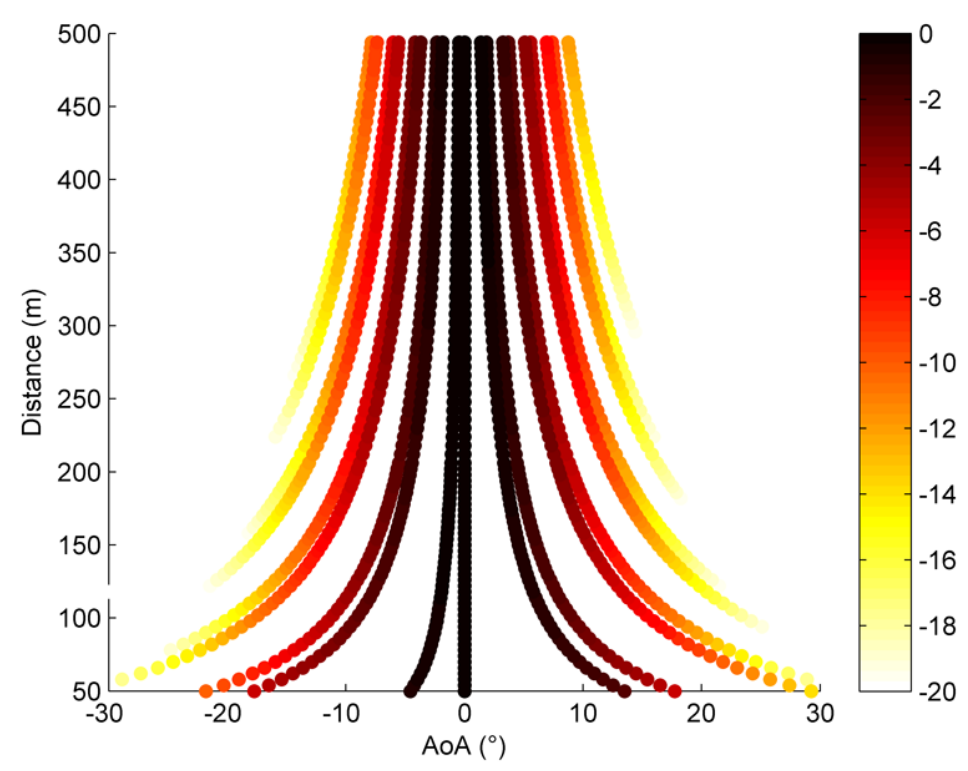

Figure 4.15. Normalized theoretical APS $(d, \varphi)$, in $\mathrm{dB}$, related to the angle of arrival (AoA).

There is no axial symmetry since the Tx and Rx antennas are situated at a transverse distance of $1 / 4$ of the tunnel width, as in the case in the experiments. Figure 4.15 clearly shows a decrease in the angle of arrival (AoA) with distance. For example, if one consider the rays having a relative attenuation equal to or smaller than $20 \mathrm{~dB}$, the AoAs are between $\pm 30^{\circ}$ if $d=50 \mathrm{~m}$ but decreases to $\pm 10^{\circ}$ when $d=400 \mathrm{~m}$.

In order to optimize all the parameters of the narrow band high resolution algorithm, one can also deduce $\operatorname{APS}(d, \varphi)$ from the theoretical channel transfer functions $H(d, i, j, f)$ at each distance $\mathrm{d}$ for the $12 \times 12$ possible links. By conducting a parametric study, it appears that the best results, shown in Figure 4.16 were obtained with 146 frequency points, in a $200 \mathrm{MHz}$ band (between 4.8 and $5 \mathrm{GHz}$ ). One can mention that the results on the APS of the angle of departure (AoD) are quite similar to those of the AoA.

As shown in Figure 4.16, the accuracy in determining the successive paths is rather good for distances up to $150 \mathrm{~m}$ but beyond this distance, it becomes more difficult to extract individual rays. This can be explained by the number of rays arriving during a short time window which become very large when the distance increases, as outlined in Section 4.3.2.2. This is the main difficulty when using high resolution algorithms to extract the AoA/AoD in such perfectly guiding structure without obstacles. These algorithms are usually applied to determine the position of the first or last interaction point (reflection or diffraction) between the rays and an obstacle, but with a rather small number of rays.

One can also recall that the antennas were placed at a height of $1 \mathrm{~m}$ from the ground. At large distances, taking this small height into account, the direct ray cannot be separated from the single- 


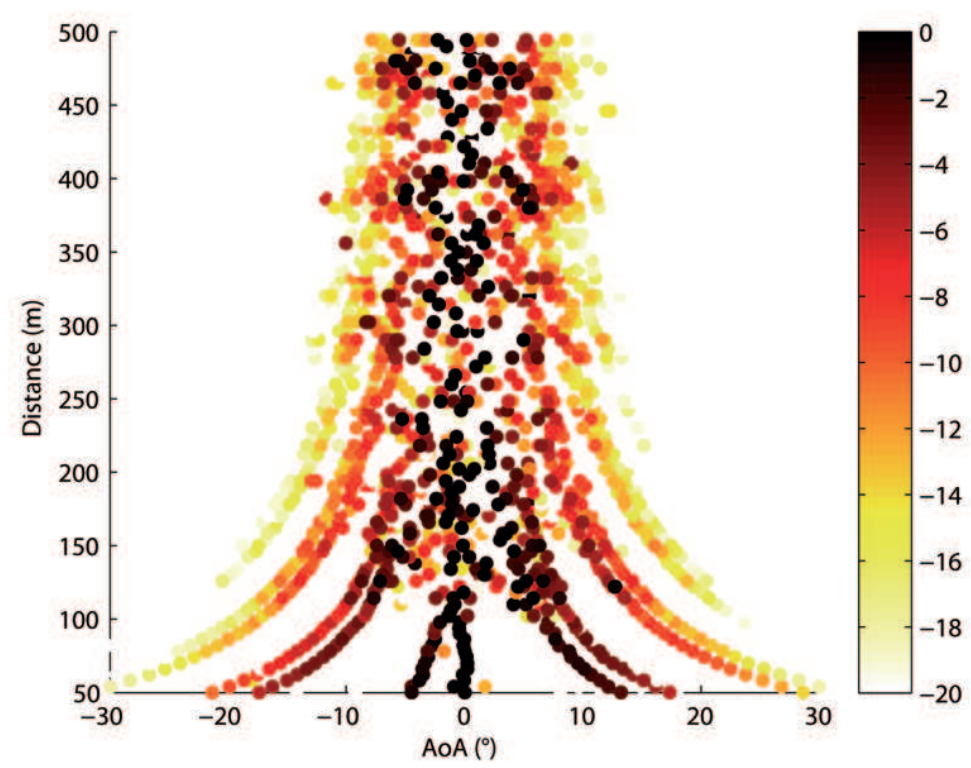

Figure 4.16. Normalized theoretical $\operatorname{APS}(d, \varphi)$, in $\mathrm{dB}$, related to the angle of arrival (AoA) and obtained by the high resolution algorithm from the theoretical channel transfer functions.

bounce ray corresponding to ground specular reflection. Despite this difficulty, such an approach would allow getting quantitative experimental values of the angular spread versus the distance between Tx and Rx.

The APS $(d, \varphi)$ of the AoA deduced from the measured channel transfer functions is given in Figure 4.17. It was found the well known result that the APS decreases with the distance but the spread of the AoA in this arched tunnel is qualitatively smaller than for the theoretical case of a rectangular tunnel (Figure 4.16). This agrees with a similar statement made in Section 4.3.2.2 about the delay spread.

It is also noted a slight negative biasing of the AoA which can probably due to an inaccuracy when positioning the fixed virtual array.

To quantitatively establish the variation of the spread of the AoA/AoD versus distance, the angular spread was calculated. This suitable measure of the extent of dispersion in direction related to the AoA is given by (see also Appendix B, Section B.5.5):

$$
A o A^{r m s}(d)=\sqrt{\frac{\sum_{k} \operatorname{APS}\left(d, \varphi_{k}\right) \varphi_{k}^{2}}{\sum_{k} A P S\left(d, \varphi_{k}\right)}-\left(\frac{\sum_{k} A P S\left(d, \varphi_{k}\right) \varphi_{k}}{\sum_{k} \operatorname{APS}\left(d, \varphi_{k}\right)}\right)^{2}}
$$

where $\varphi_{k}$ refers to the AoA of path $k$. A similar formula applies to the AoD. It should be taken into account that the average impinging power $\operatorname{APS}(d, \varphi)$ is considered to be highly confined around a 


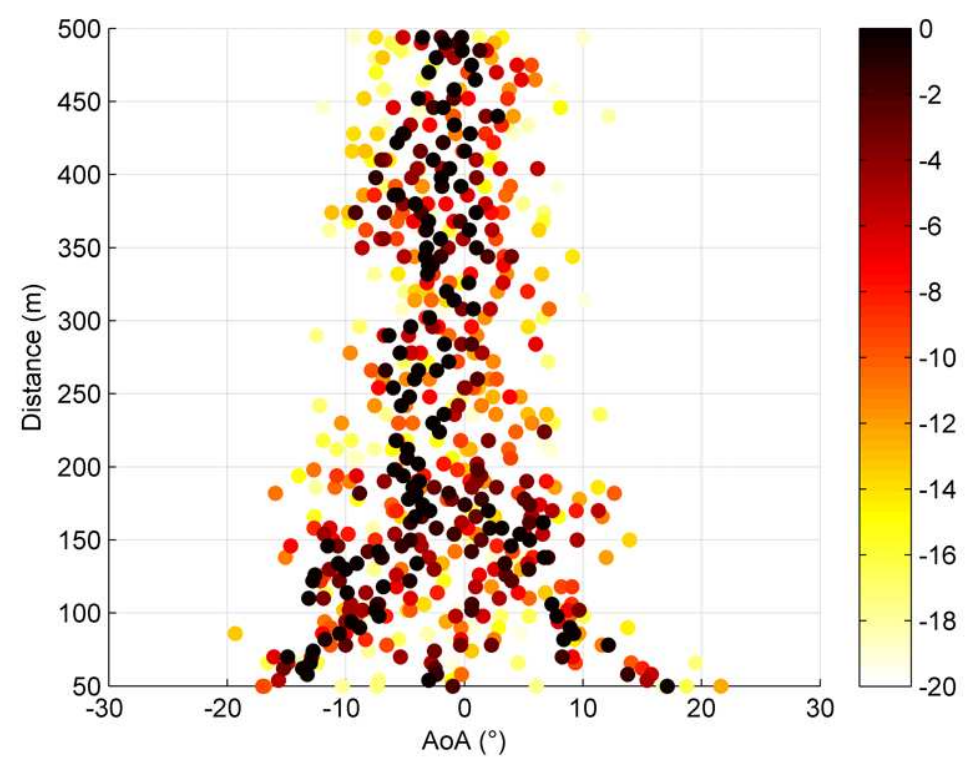

Figure 4.17. Normalized APS $(d, \varphi)$ of the angle of arrival (AoA) expressed in dB deduced from measurements.

certain azimuth. In other cases, ambiguity may appear because of the periodicity of the azimuthal angle [Molisch, 2011], [Eggers, 1999].

In Figure 4.18, one can observe how the variations in $A o A^{r m s}$ and $A o D^{r m s}$ versus distance are quite similar. The angular spread decreases from $10^{\circ}$ at $50 \mathrm{~m}$ from Tx to about $3^{\circ}$ at $200 \mathrm{~m}$ and then remains nearly constant beyond this distance.

The theoretical angular spread deduced from ray tracing in a rectangular tunnel, and assuming an infinite bandwidth (from the tap-delay model of the channel impulse response) is also plotted in Figure 4.18. In this case a continuous decrease is found since there are no constructive/destructive interactions among rays. Due to the symmetry of the problem, theoretical results are the same for AoA and AoD. The angular spread rapidly decreases from $11^{\circ}$ to $6^{\circ}$ at $200 \mathrm{~m}$, and then to $4^{\circ}$ at 500 m.

\subsubsection{Frequency Selectivity}

The selectivity of the radio channel in frequency can be evaluated by means of the channel coherence bandwidth which is a statistical measure of the range of frequencies over which the channel can be considered to be flat (see also Appendix B, Section B.5.3).

Firstly, the channel coherence bandwidth is computed versus the distance between transmitter and receiver. In order to increase the number of realizations for the statistical analysis, 36 independent channels corresponding to $6 \times 6$ positions of Tx-Rx among the $12 \times 12=144$ possible 


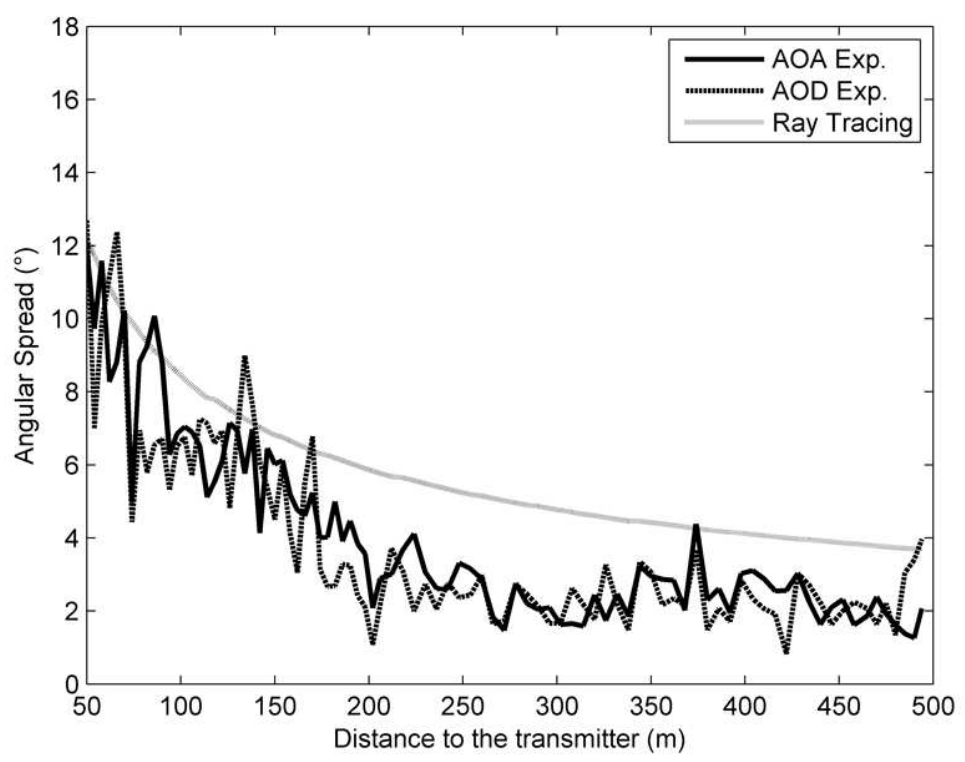

Figure 4.18. RMS angular spread versus the distance between $\mathrm{Tx}$ and $\mathrm{Rx}$, deduced from measurements (both AoA and AoD from measurements) and from a ray tracing approach (theoretical).

positions are chosen, the distance between two successive positions of $\mathrm{Rx}$ or $\mathrm{Tx}$ in the transverse plane being equal to $6 \mathrm{~cm}$. For each channel, the variation of the transfer function in a bandwidth of $1.1 \mathrm{GHz}$ is considered. In order to increase the number of transfer functions, and taking into account that the frequency sampling is $1.375 \mathrm{MHz}$, the central frequency of this interval, $f_{c}$, has been successively slide from $3.35 \mathrm{GHz}$ up to $4.45 \mathrm{GHz}$, in steps of 30 samples (41.25 MHz). This leads to 972 channels to perform a statistical analysis of the coherence bandwidth.

The $10 \%, 50 \%$ and $90 \%$ values of the Cumulative Distribution Function (CDF) of the $B_{c}$ are plotted in Figure 4.19 versus the distance between Tx and $\mathrm{Rx}$, where the coherence bandwidth has been computed for a correlation coefficient of 0.7. From these curves it appears that the median value $(50 \%)$ of $B_{c}$ increases from $40 \mathrm{MHz}$ to $550 \mathrm{MHz}$ when the distance varies from $50 \mathrm{~m}$ to $200 \mathrm{~m}$. After this point, the $B_{c}$ decreases to around $50 \mathrm{MHz}$ and increases slighly until the end of the tunnel $B_{c}(50 \%)$ being equal to $100 \mathrm{MHz}$ at $d=500$ meters.

\subsection{Diversity Techniques}

Once the channel has been characterized in space, frequency and delay domains, the diversity techniques outlined in Section 4.1.2 are evaluated experimentally. Thus, frequency, polarization, space and delay domains are considered in order to achieve the best performance of the radio link. 


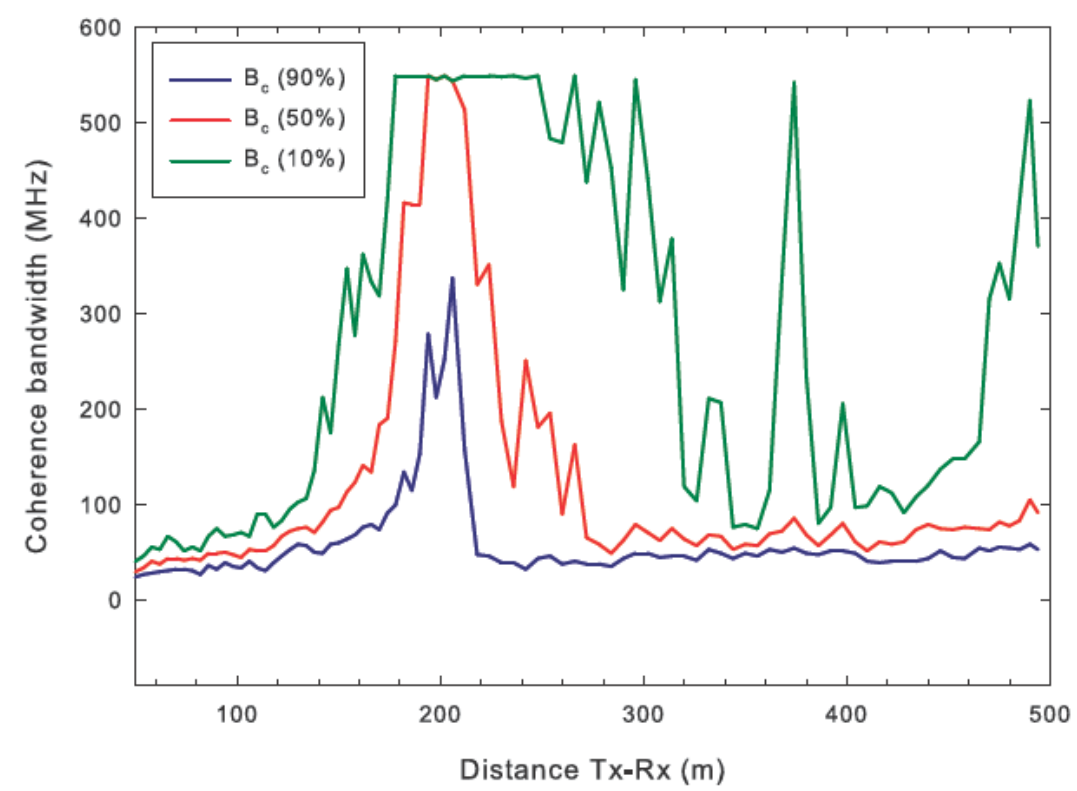

Figure 4.19. Coherence bandwidth $(\rho=0.7)$ versus distance calculated for different values of the correlation coefficient.

\subsubsection{Frequency Diversity}

As outlined in Section 4.1.2.1 if the channel is enough frequency selective, the frequency domain can also be exploited to improve the reliability of the radio link or to increase the throughput of the communications.

The analysis of the channel coherence bandwidth (the frequency selectivity of the channel is given by the channel characteristics) carried out in Section 4.3.2.4 has shown a low value of the channel coherence bandwidth for the first 150 meters along the tunnel. This fact leads to a high selectivity in frequency domain at the beginning of the tunnel.

The frequency diversity gain, $D_{f}$, using a total bandwidth $B_{p}$ of $500 \mathrm{MHz}$ is depicted in Figure 4.20. In this figure, one can observe how the highest frequency diversity degree is found at the beginning of the tunnel, over all in the first $100 \mathrm{~m}$ as expected. It decreases dramatically at $200 \mathrm{~m}$, where the highest values of coherence bandwidth were found, and remains approximately constant at 8 up to $500 \mathrm{~m}$.

In terms of diversity, the benefit achieved by frequency diversity in tunnels is unclear due to the low number of independent sub channels. 


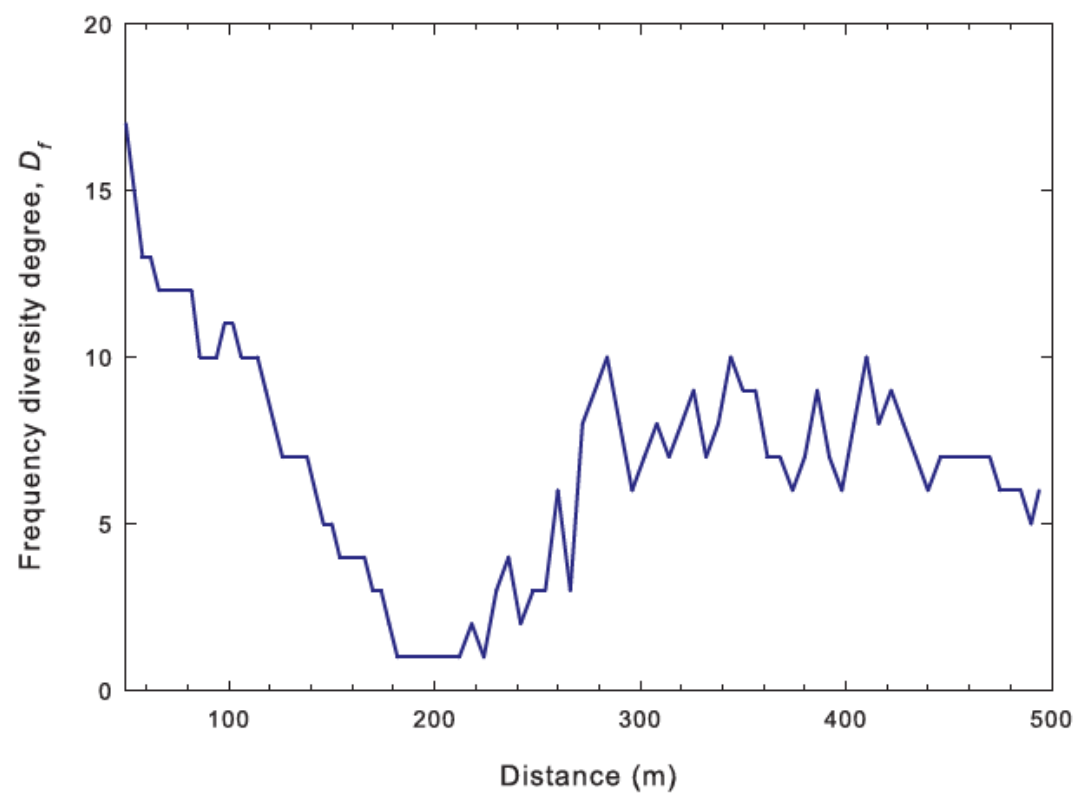

Figure 4.20. Frequency diversity degree versus distance for a total band used of $B_{p}=500 \mathrm{MHz}$.

\subsubsection{Space-Frequency Diversity}

Recent and future wireless systems combine MIMO techniques with OFDM. In Rayleigh fading channels, the channel capacity can be improved by using space-frequency block coding (SFBC) [Lee and Williams, 2000], where the Alamouti scheme is implemented over adjacent uncorrelated subbands within the same OFDM symbol. As shown previously, the flat fading channel condition in tunnel could represent a limitation for the use of such scheme.

The problem encountered in tunnel is the strong correlation in both space and frequency domains, specially for adjacent subcarriers. To overcome this drawback, a solution is to interleave the sub band in such manner that data stream is split among uncorrelated sub channels. Therefore, it could be interesting to evaluate the optimum interleaving depth for a given element spacing. To achieve this goal, the correlation in the frequency and spatial domain is studied by calculating the complex correlation function defined as:

$$
\rho_{T X}(p \Delta x, q \Delta f)=\frac{\sum_{k=1}^{6} \sum_{m=1}^{6} \sum_{n=1}^{31}\left(H\left(t x_{k}, r x_{m}, f_{n}\right) H^{*}\left(t x_{k}+p \Delta x, r x_{m}, f_{n}+q \Delta f\right)\right)}{\sqrt{\sum_{k=1}^{6} \sum_{m=1}^{6} \sum_{n=1}^{31}\left|H\left(t x_{k}, r x_{m}, f_{n}\right)\right|^{2} \sum_{k=1}^{6} \sum_{m=1}^{6} \sum_{n=1}^{31}\left|H^{*}\left(t x_{k}+p \Delta x, r x_{m}, f_{n}+q \Delta f\right)\right|^{2}}}
$$

where $t k_{k}$ is the $k t h$ transmitting antenna element, $r x_{m}$ is the $m t h$ receiving antenna element, and $f_{n}$ is the $n$th frequency. Note that $f_{n}-f_{n-1}=1.375 \mathrm{MHz}$ with $f_{1}=2.8 \mathrm{GHz}$. The distance between two successive positions of Tx and $\mathrm{Rx}$ in the transverse plane are equal to $3 \mathrm{~cm}$ and $6 \mathrm{~cm}$ respectively. $\Delta x$ 
and $\Delta f$ are the space and frequency sampling equal to $3 \mathrm{~cm}$ and $1.375 \mathrm{MHz}$ respectively. $\Delta x$ and $\Delta f$ are integer varying from 0 to 5 and 0 to 181 respectively.

Figure 4.21 shows a 3D representation of $\rho_{T X}$ using contour plot for a distance Tx-Rx equal to $50 \mathrm{~m}$ (short distance). Each curve is associated to a value of the correlation coefficient, abscissa and ordinate being related to the element spacing $p \Delta x$, and frequency separation, $q \Delta f$ respectively. If a correlation coefficient smaller than 0.7 is chosen, this value is obtained for element spacing greater than $5 \mathrm{~cm}$ and for a frequency separation equal to the frequency sampling of $1.375 \mathrm{MHz}$. In MIMO configuration, the element spacing is usually greater than this value, thus no frequency interleaving is necessary for short distances.

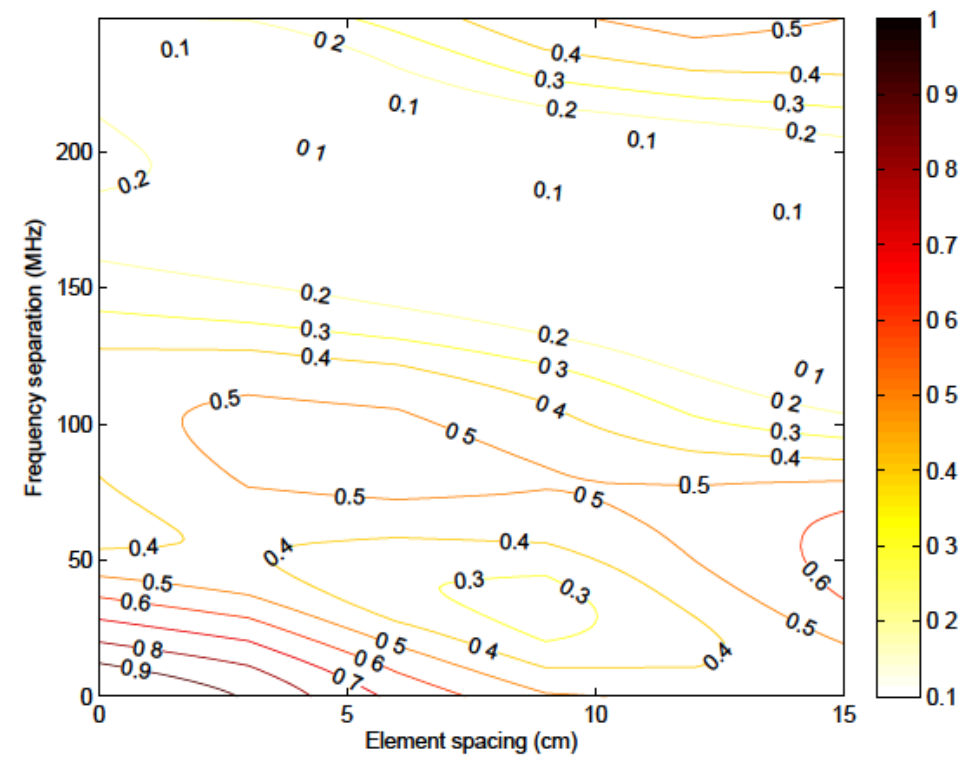

Figure 4.21. Correlation coefficient versus frequency separation and antenna element spacing for distance $T x-R x=50 \mathrm{~m}$.

The correlation coefficient calculated at $446 \mathrm{~m}$ from the transmitter (nearly the end of the tunnel) is displayed on Figure 4.22 As before, if a correlation coefficient of 0.7 is considered, the minimum bandwidth separation is $80 \mathrm{MHz}$ whatever the antenna element spacing. Thus, the frequency separation is quite large compared to the previous case (begginning of the tunnel).

Figure 4.21 and Figure 4.22 reveals that at short Tx-Rx distance in tunnel, the channel is sufficiently frequency selective and spatially uncorrelated for the use of space frequency and diversity technique. Unfortunately, at large distances, this assumption is not valid any more. 


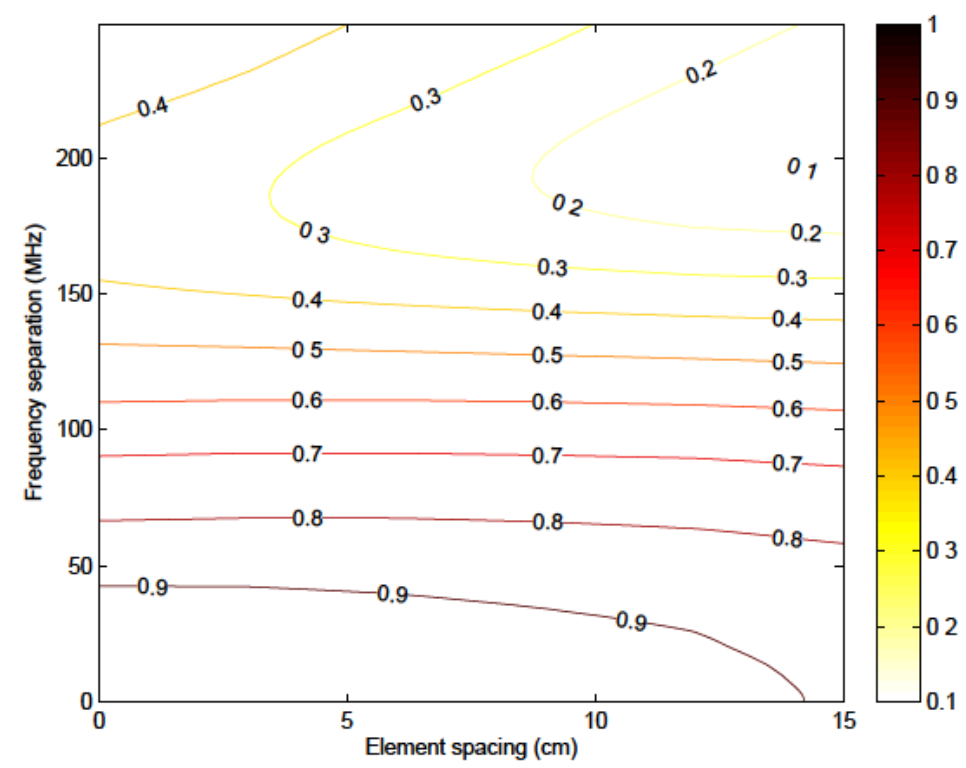

Figure 4.22. Correlation coefficient versus frequency separation and antenna element spacing for distance $T x-R x=446 \mathrm{~m}$.

\subsubsection{Polarization Diversity}

In this section, the benefits of using different polarizations scheme for MIMO communications are studied. The cases of constant received power and constant transmitted power (as in Chapter 3 Section 3.5 for and office environments) are successively considered. For this analysis the five polarizations for the arrays described in Section 4.2.3 are considered. The results are presented below.

\subsubsection{Capacity for Constant Received Power}

In this case, MIMO capacity only depends on the properties of each transfer matrix $H(d, f)$, deduced from the measured matrix $G(d, f)$ by Frobenius normalization [Foschini and Gans, 1998].

The average complex correlation coefficients $\rho_{T x}^{p, q}(d, f)$ between the electric fields transmitted by the Tx elements $p$ and $q$, at a distance $d$ was calculated for VV polarization in Section 4.3.2.1 and depicted in Figure 4.9. It was shown that for the first $100 \mathrm{~m}$ along the tunnel, the correlation at the transmitter remains low while it increases after this distance. If the antenna spacing was larger, the correlation distance increased. This increment of the correlation with distance can be explained through the modal theory of the electromagnetic wave propagation, high order hybrid modes being strongly attenuated at large distances [Molina-Garcia-Pardo et al., 2008b]. This behavior was also observed for $\mathrm{HH}$ configuration.

A possible way of decreasing the correlation between successive elements is to introduce polarization diversity, using, for example, a VHVH array as mentioned in Section 4.2.3 In this case, 
the mean correlation coefficient is lower than for VV configurations, as well as, it tends to decrease with distance.

Given the fact that the correlation for VHVH configuration is much smaller than for VV arrays, one can expect an increase in the capacity in this case. Thus, the capacity of the multiantenna system can be computed as:

$$
C(d, f)=E_{H}\left[\log _{2}\left(\operatorname{det}\left(\mathbf{I}_{\mathbf{N}}+\frac{S N R}{M} \mathbf{H H}^{\mathbf{\dagger}}\right)\right)\right]
$$

where $\mathbf{I}_{\mathbf{N}}$ is the $N \times N$ identity matrix, + represents the conjugate transpose operation, $\mathbf{H}$ is the Frobenius normalized $G$ matrix, and SNR is the signal to noise ratio at the receiver. The average capacity in the frequency band under consideration, $C(d)$, was then computed by averaging $C(d, f)$ over frequency.

The variation of the cpaacity for the five polarization configurations described Section 4.2 .3 and for a constant SNR of $15 \mathrm{~dB}$, is represented in Figure 4.23

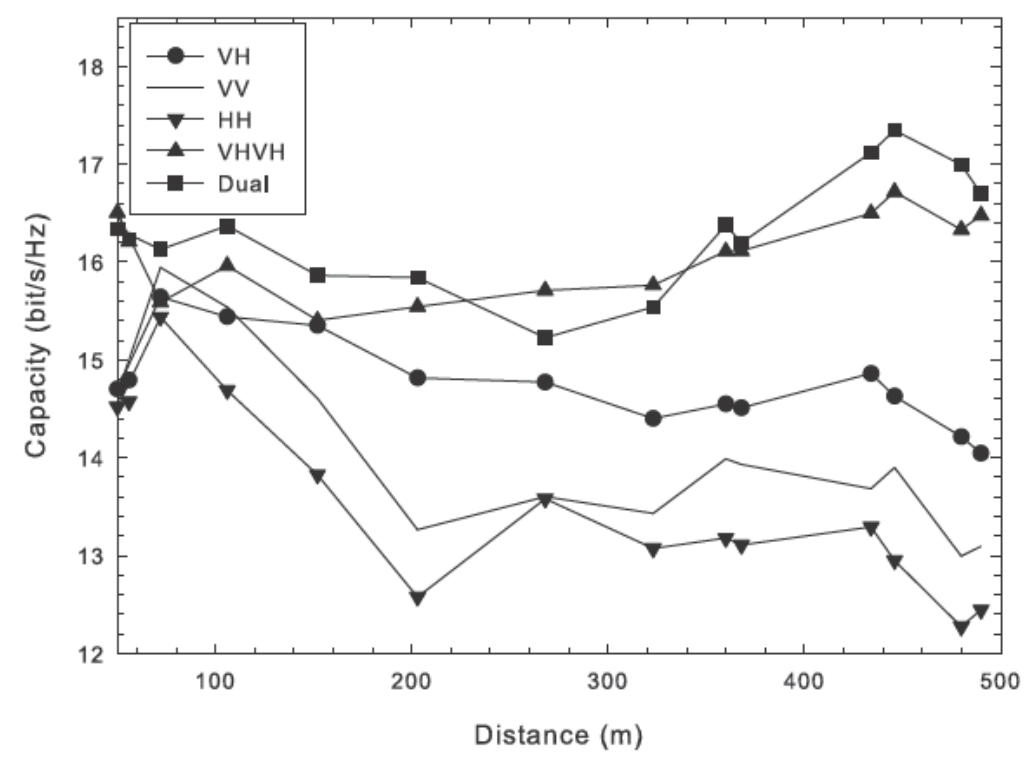

Figure 4.23. Mean MIMO capacity assuming a fixed SNR at the receiver of $15 \mathrm{~dB}$.

As expected, there is a strong dependence between capacity and correlation, the worst configurations being VV and HH. For VV co-polarized elements, the capacity decreases from 16 $\mathrm{bits} / \mathrm{s} / \mathrm{Hz}$ at a short distance from Tx to $13 \mathrm{bits} / \mathrm{s} / \mathrm{Hz}$ for $d=500 \mathrm{~m}$. On the contrary, VHVH and Dual give the best performances, with a capacity on the order of $16-17 \mathrm{bits} / \mathrm{s} / \mathrm{Hz}$, whatever the distance. It can be interesting to mention that the maximum theoretical capacity of a $4 \times 4 \mathrm{MIMO}$ i.i.d. channel would be around $20 \mathrm{bits} / \mathrm{s} / \mathrm{Hz}$. Since MIMO is a rather expensive technology, it is also very important to compare its performances to those of a simple Single-Input Single-Output (SISO) 
system. As a constant SNR of $15 \mathrm{~dB}$ is assumed, the capacity of a SISO link would be $5 \mathrm{bits} / \mathrm{s} / \mathrm{Hz}$, thus much smaller than for MIMO.

However, in practical cases corresponding to a fixed transmitting power; the capacity depends on the received power. It is thus important to study the received power as a function of the polarization. Indeed, the channel capacity of the MIMO link can be improved by introducing polarization diversity only if the decrease in correlation between arrays elements can balance a possible loss of received power on the cross polarized elements. This tradeoff between SNR and correlation was outlined in [Khan and Hall, 2010] for other types of channel.

\subsubsection{Capacity for Constant Transmitted Power}

In this case the measured MIMO capacity depends not only on the channel characteristics but also on the SNR at the receiver, and thereby on the measured received power. Such received power depends also on the array configurations.

To outline the influence of the polarization on the received power $P_{r}$, it is thus interesting to smooth the small scale variation of $P_{r}$, by averaging its value over the whole frequency range and over the successive positions of the antennas in the transverse plane. Curves in Figure 4.24 give the average received power $P_{r, a v}$ versus distance, $P_{r, a v}$ being normalized to the power received at $50 \mathrm{~m}$ for a VV polarization.

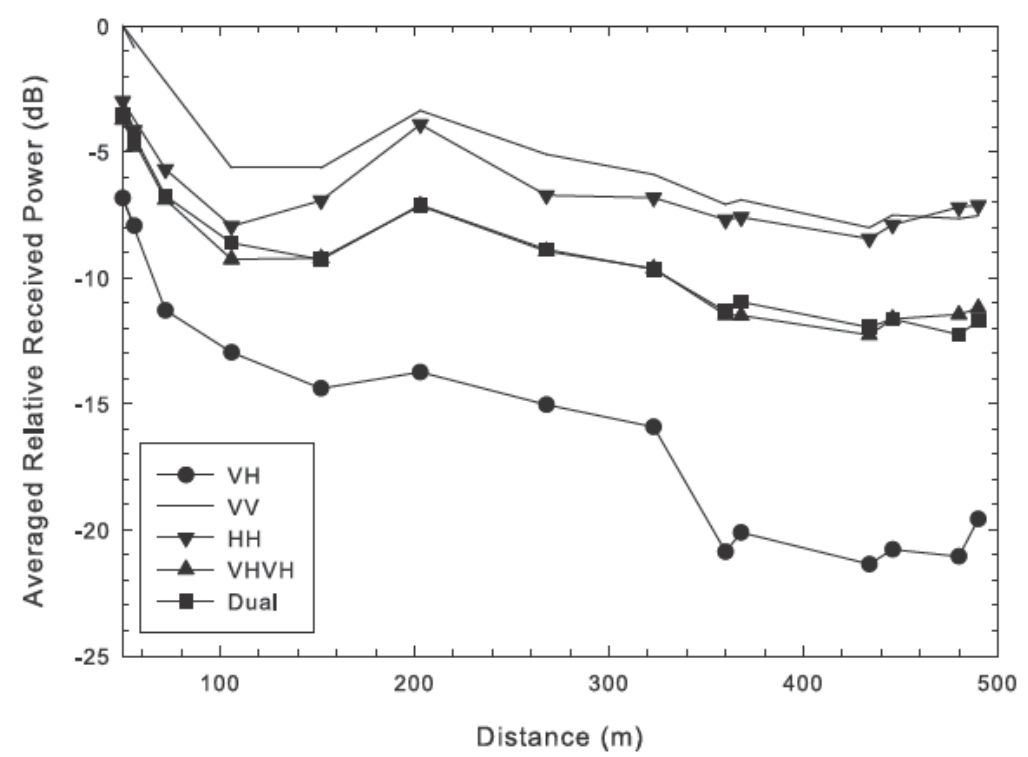

Figure 4.24. Average relative received power for various polarizations. The reference value is 0 $\mathrm{dB}$ at $50 \mathrm{~m}$ and for VV polarization. 
Firstly, if a co-polar transmission is considered, one can see that $P_{r, a v}$ does not strongly vary between $100 \mathrm{~m}$ and $500 \mathrm{~m}, \mathrm{VV}$ giving nevertheless slightly better results than $\mathrm{HH}$. For the full cross polar configuration (VH), the received power is much smaller. The XPD (Cross-Polarization Discrimination Factor) increases with distance, and it varies from $7 \mathrm{~dB}$ near $\mathrm{Tx}$ to reach $15 \mathrm{~dB}$ if $d=500 \mathrm{~m}$. This means that the waves remain strongly polarized in such arched tunnels. Finally, for the last two cases, VHVH and Dual, half of the elements of the MIMO matrix are co-polarized, while the others are cross-polarized. As a result, the mean received power is about $3 \mathrm{~dB}$ less than for VV co-polarization.

Once the measured received power has been analyzed for all polarization, the measured capacity for constant transmitted power can be computed. To make a numerical application, we have chosen a reference point corresponding to a SNR of $15 \mathrm{~dB}$ for the VV configuration and a distance $d=500$ $\mathrm{m}$. The average SNR versus distance and for the various polarizations is directly deduced from the curves in Figure 4.24

The average capacity in the frequency band, $C(d)$, is plotted in Figure 4.25 The VH curve obviously shows that full cross-polar is not suitable in a tunnel, the loss in terms of received power being too important. For the two other configurations of diversity polarization, namely VHVH and Dual, the capacity is nearly the same as for the $\mathrm{HH}$ polarization, the decrease in the correlation between the Tx and Rx elements compensating the loss of received power. Capacity obtained with such MIMO configurations is much larger that the SISO capacity for vertical polarization, as also shown in this Figure.

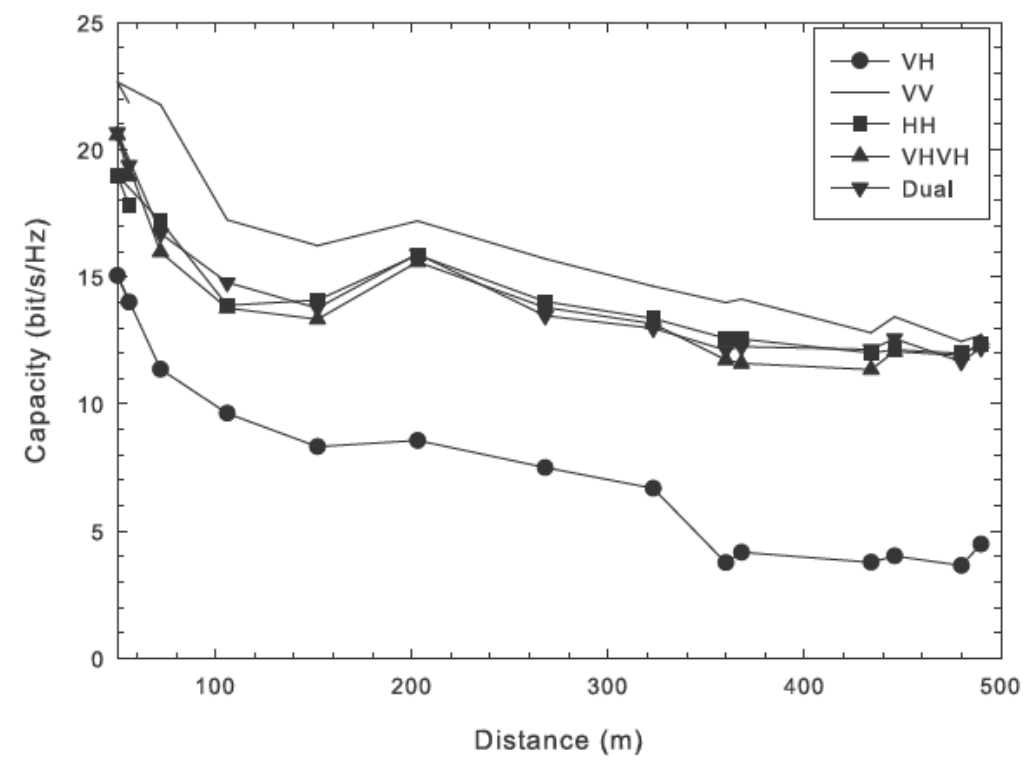

Figure 4.25. Mean MIMO capacity assuming a fixed Tx Power. 
Finally, the best performances are obtained for vertical co-polarized elements, the average received power being slightly higher than for $\mathrm{HH}$ (around $1 \mathrm{~dB}$ ).

Consequently, changing the polarization of the successive elements of the antenna arrays, as for VHVH, does not present an interest for improving MIMO performances in an arched tunnel. Concerning the Dual configuration, it is important to recall that the distance between the two dualpolarized array elements is $18 \mathrm{~cm}$, i.e. the total length of the array chosen as a reference. The interest of using Dual could be to decrease this length while keeping the same channel capacity. As an example, let us consider the most critical case in terms of correlation between array elements corresponding to a large distance between $\mathrm{Tx}$ and $\mathrm{Rx}$.

Table 4.2 gives the average capacity between $400 \mathrm{~m}$ and $500 \mathrm{~m}$, first for co-polarized vertical elements (VV), the length of the array being either $18 \mathrm{~cm}$ or $9 \mathrm{~cm}$ and, secondly, for dual-polarized elements arrays of different lengths. The other parameters of the link are identical to those of the previous sections. We see that using dual-polarized elements allows decreasing the total length of the arrays without loosing capacity.

\begin{tabular}{||c|c|c|c|c|c|c||}
\hline \hline Configuration & \multicolumn{2}{|c|}{ VV } & \multicolumn{3}{c||}{ Dual-polarized elements } \\
\hline Length & $18 \mathrm{~cm}$ & $9 \mathrm{~cm}$ & $3 \mathrm{~cm}$ & $6 \mathrm{~cm}$ & $9 \mathrm{~cm}$ & $18 \mathrm{~cm}$ \\
\hline Capacity & 12.8 & 10.0 & 9.4 & 10.6 & 11.5 & 12.2 \\
\hline \hline
\end{tabular}

Table 4.2. Average value of the capacity far from the transmitter using either co-polarized vertical antennas or dual-polarized antennas of different lengths.

\subsubsection{Space-Delay Diversity: Time Reversal}

As mentioned in Section 4.1.2 Time Reversal can be seen as a kind of delay-space diversity technique. In this section the benefits of using the Time Reversal technique described in Section 4.1.2.3 in tunnels are evaluated for both SISO and Multiple-Input Single-Output (MISO) configurations. However, firstly it is necessary to have comprehensive knowledge of the characteristics of the received and transmitted waveforms.

\subsubsection{Transmitted and Received Waveforms}

As previously outlined in Section 4.2, the complex channel transfer function was measured in the frequency domain between 2.8 and $5 \mathrm{GHz}$. The equivalent transmitted pulse is represented in Figure 4.26a.

It has been obtained by applying on the complex transfer function, measured in the frequency domain, a Hamming window and an inverse Fourier transform. The maximum amplitude of the pulse is normalized to $1 \mathrm{~V}$. The pseudo frequency corresponds to the center of the frequency band 


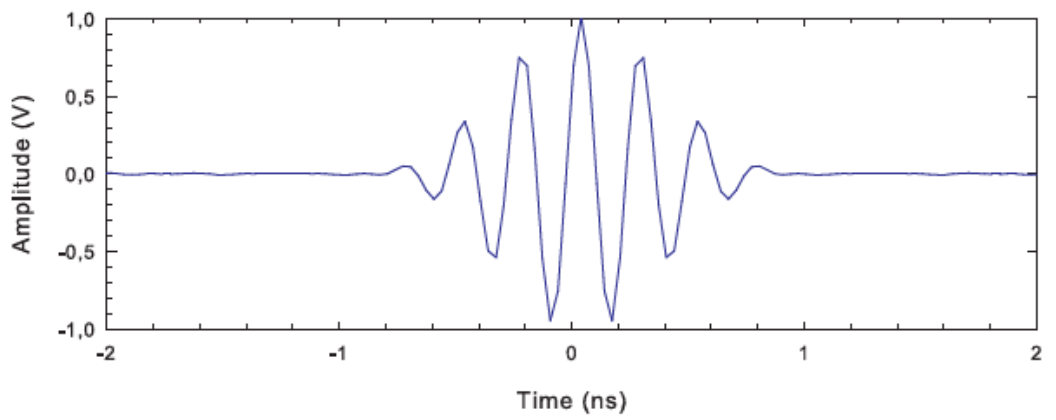

(a)

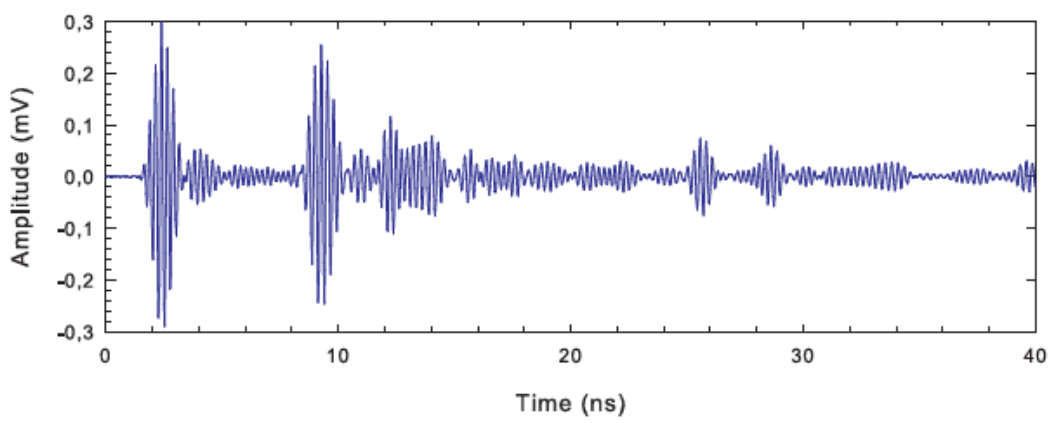

(b)

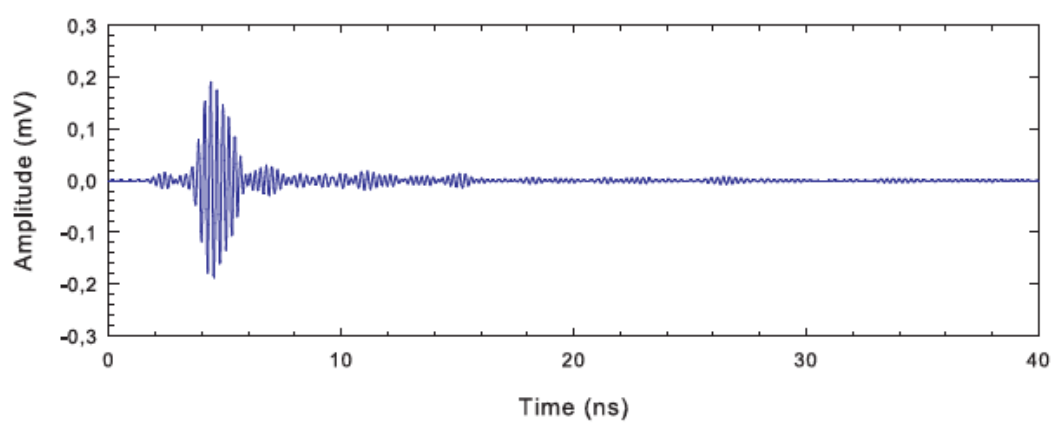

(c)

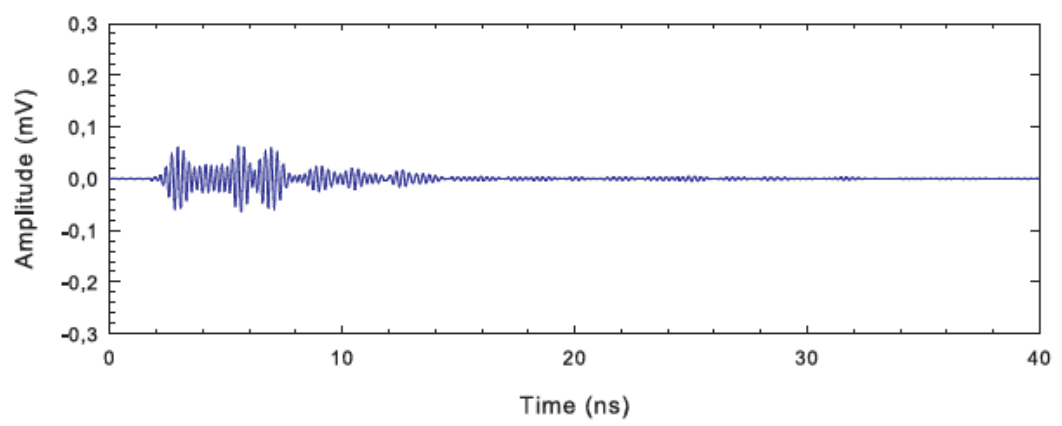

(d)

Figure 4.26. Waveforms of the (a) transmitted pulse, and (b) to (d) of the received signals at different distances. 
under analysis $(3.9 \mathrm{GHz})$. Examples of the received signal waveform at a distance of $50 \mathrm{~m}, 200 \mathrm{~m}$ and $498 \mathrm{~m}$ are given in Figure $4.26 \mathrm{~b}$, Figure $4.26 \mathrm{~d}$ and Figure $4.26 \mathrm{~d}$, To avoid time shift due to the propagation delay, the origin of the time axis in this figure, and in the following figures, is quite arbitrary.

At $d=50 \mathrm{~m}$, one can observe a first pulse, which includes the direct path, and which has a peak amplitude of $0.3 \mathrm{mV}$. Then, there is a series of other delayed corresponding to multipath propagation. For a delay of $30 \mathrm{~ns}$, the maximum amplitude of the pulse is $0.07 \mathrm{mV}$, which is not still negligible, referred to the amplitude of the strongest pulse. At $d=200 \mathrm{~m}$, the first received pulse has a width of about $2 \mathrm{~ns}$ and has nearly the same amplitude and shape as that obtained at $50 \mathrm{~m}$, but the amplitudes of the delayed pulses are very small. This result can be explained from a theoretical modeling of the propagation in a straight tunnel, based on a ray theory [Molina-Garcia-Pardo et al., 2008b]. Due to the finite bandwidth (even if it is equal to $2.1 \mathrm{GHz}$ ), interferences between rays reflecting on the tunnel walls occur. The total signal can be arbitrary put in the sum of two contributions: on the one hand the direct path and rays reflecting on the walls with a grazing angle of incidence and, on the other hand, rays experiencing many reflections on the walls. It numerically appears from the theoretical model that, for a distance between the transmitter and the receiver on the order of $200 \mathrm{~m}$, destructive interference occurs between rays of high order reflection, leading in the time domain to delayed pulses of small amplitude. This critical distance of $200 \mathrm{~m}$ in our case, of course depends on the tunnel geometry.

For a distance equal to $498 \mathrm{~m}$, the curve plotted in Figure 4.26d shows a high multipath density, but within a short time delay, of about $10 \mathrm{~ns}$. Indeed at large distance, the main contribution to the signal is due to rays propagating nearly parallel to the tunnel axis [Molina-Garcia-Pardo et al., 2008b]. It is thus interesting to determine the correlation function between signals received at different locations to be able to interpret the results which would be obtained with Time Reversal.

\subsubsection{Time Reversal}

Let us first consider the SISO configuration, with a single antenna being used at the Tx site and at the Rx site. The implementation of TR involves the previous estimation of the CIR. Let us assume a bidirectional and stationary channel where the transmitter and receiver are separated by a distance $d_{0}$. Let us also assume that the CIR has been estimated. This response can then be inverted and conjugated, $h^{*}\left(d_{0}, \tau\right)$, and used as the pre-coding of the transmitted UWB pulse [Lerosey et al., 2004], [Lerosey et al., 2005]. Note that in our case of IR UWB, the channel impulse response (CIR) is real and the conjugate operation is not required. If the transmitted pulse is noted $p(\tau)$, the received signal at any distance $d$ from the transmitter, $y_{T R}(d, \tau)$ can be expressed as follows:

$$
y_{T R}(\tau)=\left(p(\tau) \otimes h^{*}\left(d_{0},-\tau\right)\right) \otimes h(d, \tau)=p(\tau) \otimes\left(h(d, \tau) \otimes h^{*}\left(d_{0},-\tau\right)\right)=p(\tau) \otimes h_{e q}(d, \tau)
$$


The equivalent CIR, using TR is thus given by:

$$
h_{e q}(d, \tau)=h(d, \tau) \otimes h^{*}\left(d_{0},-\tau\right)
$$

Since in our study of propagation in tunnels and measurements were carried out in the frequency domain, (4.16) can also be written as:

$$
y_{T R}(d, \tau)=\operatorname{IFFT}\left\{P^{*}(f) H^{*}\left(d_{0}, f\right) H(d, f)\right\}
$$

In this formula, $P(f)$ is the frequency content of the transmitted pulse, and $H\left(d_{0}, f\right)$ and $H(d, f)$ are the complex pass band transfer functions for both positive and negative frequencies, deduced from the measurement results.

In practical systems, rather than evaluating the intrinsic channel transfer function, the precoding signal used at the transmission site is the real channel impulse $h\left(d_{0}, \tau\right)$ response convolved with the pulse $p(\tau)$ as a result of a previous demand from the transmitter to the receiver. Furthermore, to carry out a fair comparison of the received signals with or without TR, the transmission power must be the same in both cases. Taking these two aspects into account, the received signal can be put in the following final form:

$$
y_{T R}(d, \tau)=\operatorname{IFFT}\left\{\frac{P(f) P^{*}(f) H^{*}\left(d_{0}, f\right) H(d, f)}{\sqrt{\left|P^{*}(f) H^{*}\left(d_{0}, f\right)\right|^{2}}}\right\}
$$

To increase the gain when using TR, a possible solution which has already been investigated is to employ multiple antenna transmission schemes, such as MISO [Qiu et al., 2006], [Kyritsi et al., 2004], [Naqvi and El Zein, 2008]. If there are M antennas at the Tx site, the received signal is the coherent sum of all the TR signals at the target receiver and can be expressed as:

$$
y_{T R}(d, \tau)=\operatorname{IFFT}\left\{\sum_{j=1}^{M} P(f) \frac{P^{*}(f) H_{j}^{*}\left(d_{0}, f\right)}{\sqrt{M\left|P^{*}(f) H_{j}^{*}\left(d_{0}, f\right)\right|^{2}}} H_{j}(d, f)\right\}
$$

where $H_{j}\left(d_{0}, f\right)$ is the pass band transfer function between the transmitter $j$ and the target receiver. In order to point out the benefits of using TR techniques in tunnels, examples will first be given for three different distances $\mathrm{d}$ between $\mathrm{Tx}$ and $\mathrm{Rx}$ : around $50 \mathrm{~m}$ and $500 \mathrm{~m}$ corresponding to the maximum and minimum values of $d$ in the experiments, and at around $200 \mathrm{~m}$. This particular distance has been chosen since in this zone the delay spread reaches its minimum value. 


\subsubsection{Spatial Focusing of SISO-TR}

One of the advantages of Time Reversal, pointed out in the literature, is that the signal can only be optimally received by the target receiver associated with the CIR used in the pre-coding technique. In order to outline this spatial focusing, the case of an optimal pre-coding, i.e. when the CIR has been estimated at the location of the receiving point $\left(d=d_{0}\right)$ is considered. The signal obtained in this case with SISO-TR and at a distance $d=50 \mathrm{~m}$ is plotted in Figure 4.27a Let us now assume that the receiver moves to $d=54 \mathrm{~m}$ and then to $d=58 \mathrm{~m}$ but by keeping the CIR at $50 \mathrm{~m}$ for pre-coding. Since the axial correlation coefficient is equal to 0.4 between two points $4 \mathrm{~m}$ apart (see Figure 4.10), the focusing effect of the TR no longer appears as shown in Figure $4.27 \mathrm{~b}$ and Figure $4.27 \mathrm{C}$

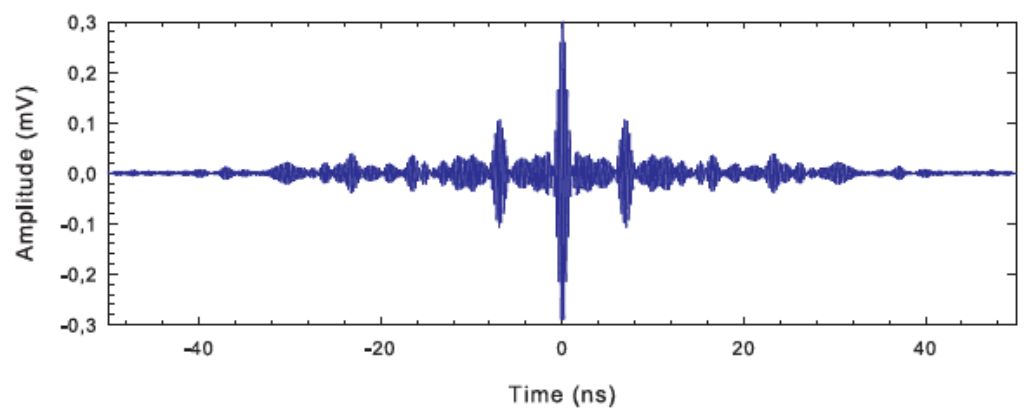

(a)

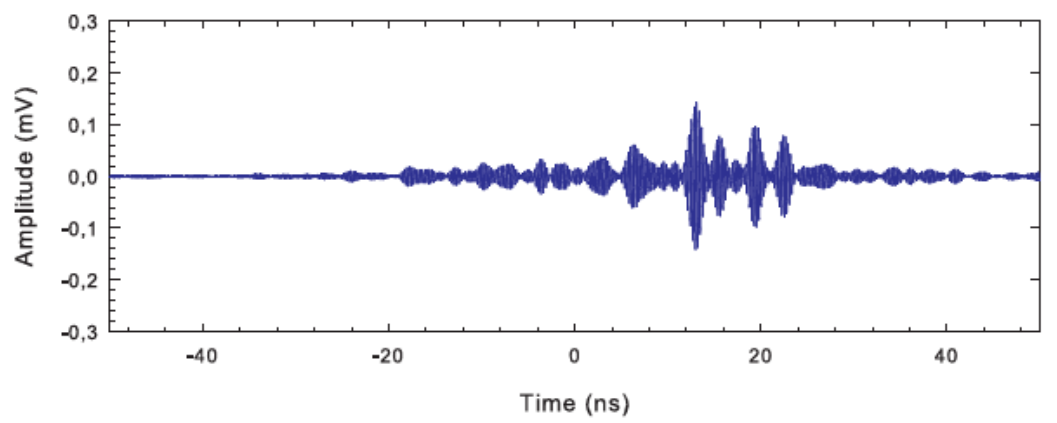

(b)

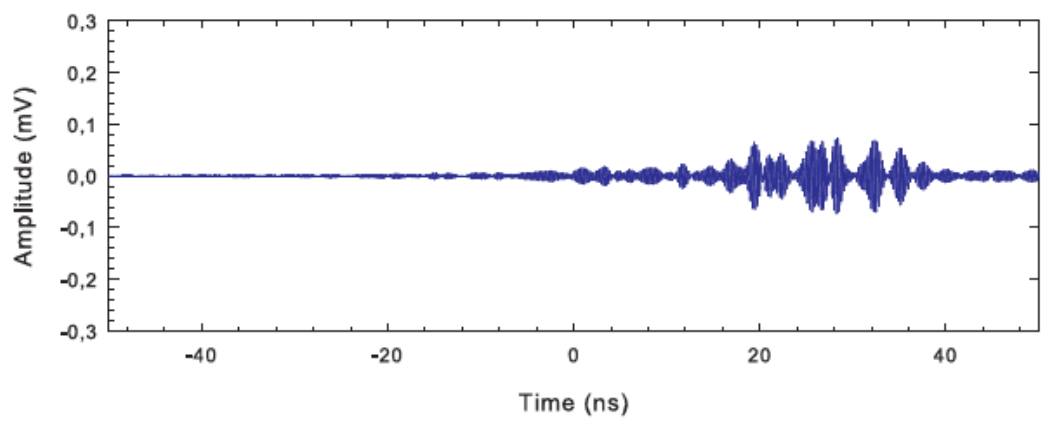

(c)

Figure 4.27. Received signal with TR: (a) $d=d_{0}=50 \mathrm{~m}$, (b) $d=54 \mathrm{~m}$ but $d_{0}=50 \mathrm{~m}$, (c) $d=58$ $\mathrm{m}$ but $d_{0}=50$. 


\subsubsection{Temporal Focusing: Comparison between SISO-TR and MISO-TR}

Before extracting some TR characteristics from all measurement results, it is interesting to present typical waveforms of the received signal. It is presumed in this section that the precoding is optimum, i.e. it uses the channel estimation at the receiving point. This means that in (4.19) $d=d_{0}$. As an example, Figure 4.28a indicates the waveform of the signal at $50 \mathrm{~m}$ with SISO TR. For this last configuration, TR is equivalent to a coherent summation of all paths, giving rise to an increase in the Rx power at and a decrease of the side lobes. All quantitative aspects will be considered in the following section.

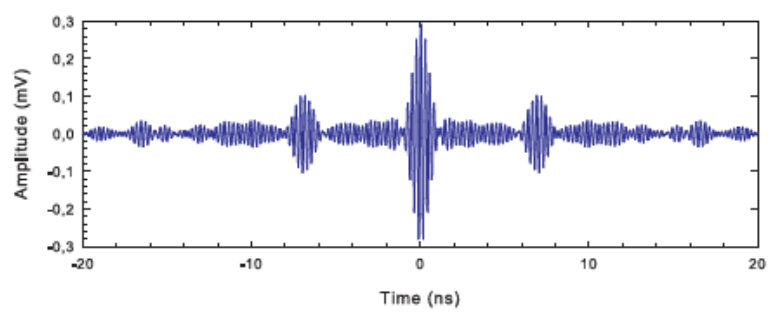

(a)

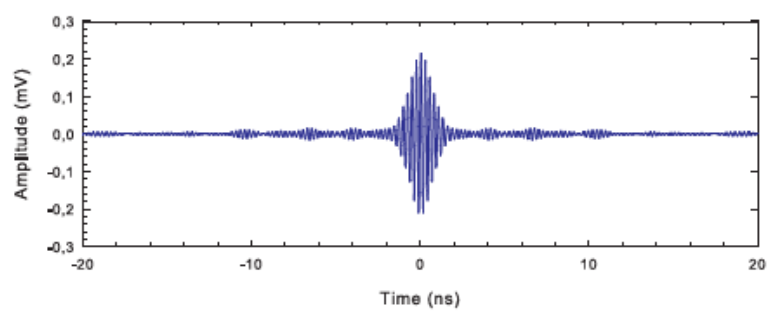

(c)

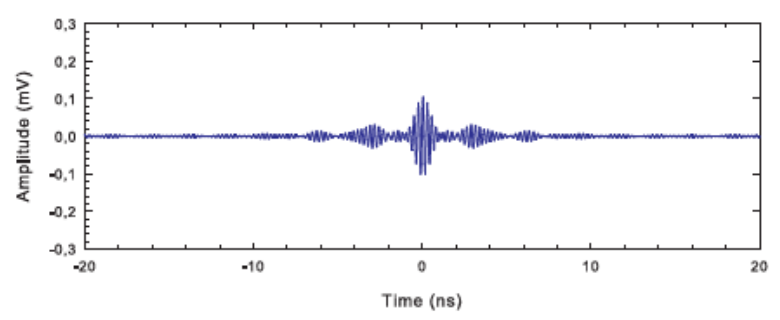

(e)

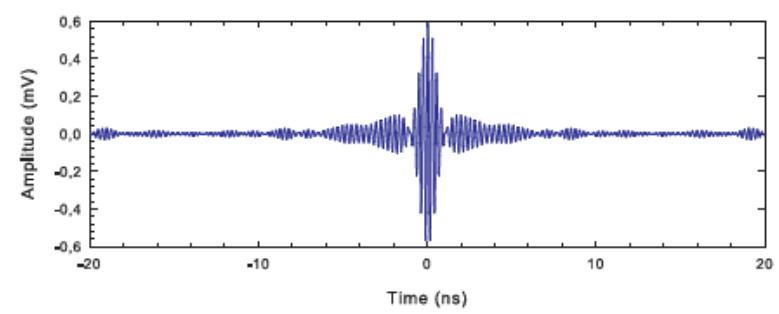

(b)

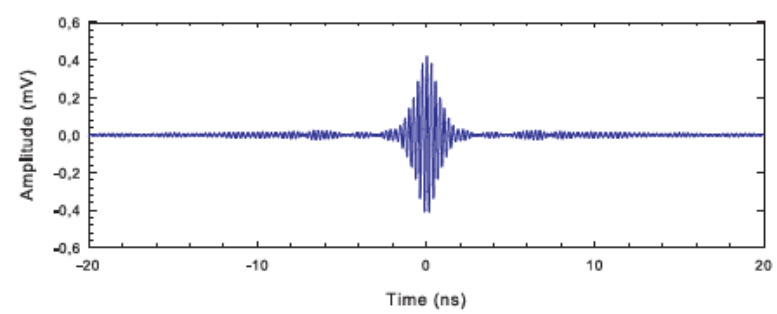

(d)

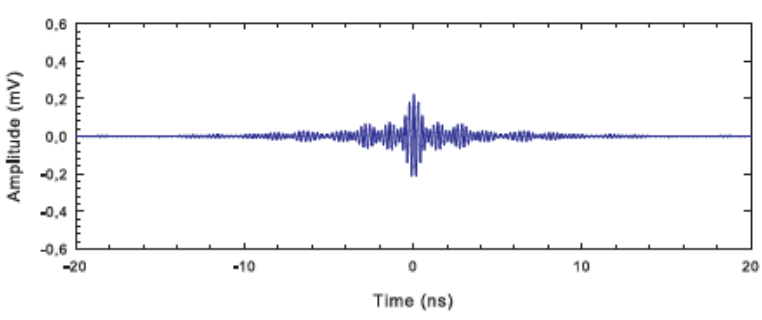

(f)

Figure 4.28. Waveform of the received signals at (a) $d=50 \mathrm{~m}$ with SISO-TR, (b) $d=50 \mathrm{~m}$ with MISO-TR, (c) $d=200 \mathrm{~m}$ with SISO-TR, (d) $d=200 \mathrm{~m}$ with MISO-TR, (e) $d=498 \mathrm{~m}$ with SISO-TR, (b) $d=498 \mathrm{~m}$ with MISO-TR.

An illustration of the application of MISO TR is given in Figure 4.28b Four Tx antennas have been considered in this case, the spacing between each antenna being $9 \mathrm{~cm}$. This corresponds to the maximum possible spacing in our experiments. Consequently, only one Tx virtual array can be considered. Comparing Figure 4.28a and Figure 4.28b a gain factor of around 2 on the peak value of the strongest signal is observed for the MISO case. As already mentioned, this is due to the coherent addition of the peaks from each branch in the MISO TR response. It has been shown that the gain 
factor on the peak of the channel response with MISO TR is higher than the peak average over all the M SISO cases [Zhou et al., 2006].

Lastly, waveforms of the signal received at a distance of $200 \mathrm{~m}$ and $498 \mathrm{~m}$ are plotted in Figure $4.28 \mathrm{~d}$ to Figure $4.28 \mathrm{f}$ As mentioned in Section 4.4.3.1 at $200 \mathrm{~m}$ the delayed multipath components of the signal without TR are strongly attenuated, the pulse having duration of about $2 \mathrm{~ns}$. Therefore, the benefit in terms of a reduction of the signal spread and in terms of gain brought about by TR is not significant, as can be shown by comparing Figure $4.26 \mathrm{C}$ and Figure $4.28 \mathrm{c}$ At 498 $\mathrm{m}$, the received pulse without TR being slightly wider than at $200 \mathrm{~m}$, the main interest of using SISO TR or MISO TR is to increase the peak value of the received pulse.

Curves presented in Figure 4.28 clearly show that the improvement which could be expected on the temporal focusing by using TR, is dependent on the distance $d$ between the transmitter and the receiver inside the tunnel. The next section is thus devoted to the variation of the focusing characteristics versus this distance.

\subsubsection{Some Time-Reversal Characteristics}

Various figures of merit are proposed in the literature to evaluate the main features of TR. The first possible metric is the average RMS delay spread $\left(D_{s}\right)$ describing the temporal compression of the signal. The three curves in Figure 4.29 have been plotted for the following configurations: SISO without TR, SISO TR and MISO TR. For MISO, the number M of transmitting elements is, as previously, equal to 4 .

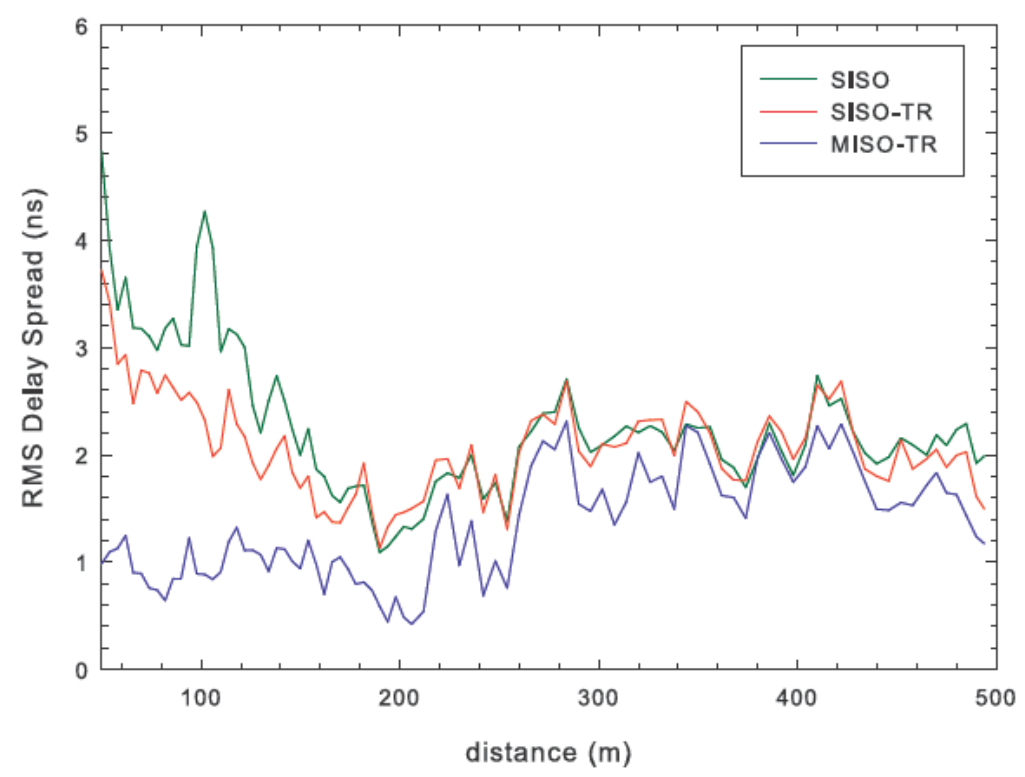

Figure 4.29. Average rms delay spread: SISO without TR, SISO TR and MISO TR. 
For each distance $d, D_{s}$ was averaged over the 12 positions of the Rx antenna in the transverse plane and over the 12 positions of the Tx antenna in the SISO case, and over the 12 positions of the Rx antenna in the MISO case. At a distance of $50 \mathrm{~m}, D_{s}$ is on the order of $5 \mathrm{~ns}$, and reduces to $3.5 \mathrm{~ns}$ with SISO TR. A noticeable improvement is obtained for MISO TR, with $D_{s}$ decreasing to 1 ns. However, the significance of using TR becomes small at large distances, since the average delay spread for the 3 configurations is quite similar. Indeed in this case, the transmitting bandwidth is not sufficiently important to profit from the multipath propagation, the delay spread without TR being only $2 \mathrm{~ns}$. To clearly indicate the reduction of $D_{s}$ by using SISO TR and MISO TR, the reduction factors of delay spread, noted $R_{S I S O-T R}$ and $R_{M I S O-T R}$ respectively and expressed in percentages, have been computed from the following expressions:

$$
\begin{gathered}
R_{S I S O-T R}(d, i, j)=100\left(\frac{D_{s}(d, i, j)-D_{s}^{S I S O-T R}(d, i, j)}{D_{s}(d, i, j)}\right) \\
R_{M I S O-T R}(d, j)=100\left(\frac{\left\langle D_{s}(d, i, j)\right\rangle_{i}-D_{s}^{M I S O-T R}(d, j)}{<D_{s}(d, i, j)>_{i}}\right)
\end{gathered}
$$

In (4.21) and (4.23), $D_{s}(d, i, j), D_{s}^{S I S O-T R}(d, i, j)$ and $D_{S}^{M I S O-T R}(d, i, j)$ are the RMS delay spreads, without TR, with SISO-TR and with MISO-TR respectively, and calculated at an axial distance $d$. The indices $i$ and $j$ characterize the location of Tx and $\mathrm{Rx}$ in the transverse plane respectively.

The curves in Figure 4.30 correspond to the Cumulative Distribution Function (CDF) of $R_{S I S O-T R}$ and $R_{M I S O-T R}$.

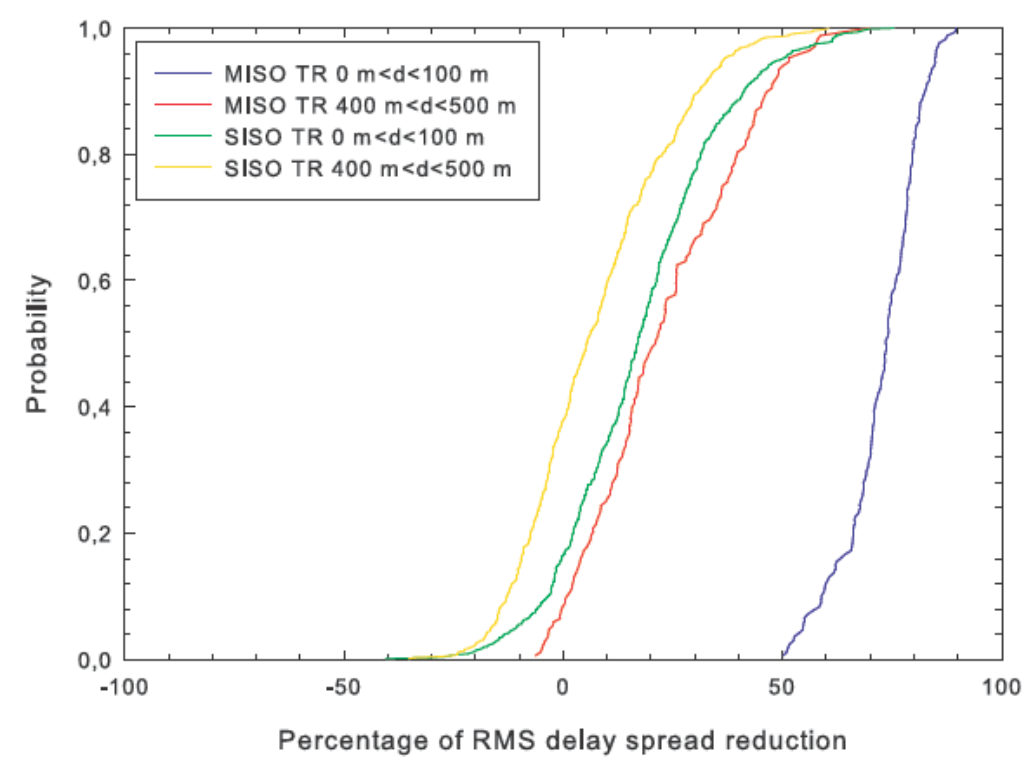

Figure 4.30. CDF of $R_{S I S O-T R}$ and $R_{M I S O-T R}$ for short range $(d<100 \mathrm{~m})$ and for long range (400 $\mathrm{m}<d<500 \mathrm{~m}$ ) communication. 
Two zones have been considered: One close to Tx (50 m $<d<100 \mathrm{~m})$, and the other far away from Tx $(400 \mathrm{~m}<d<500 \mathrm{~m})$. For a probability of 0.5 , the reduction of $D_{s}$ with SISO TR is only $15 \%$ in the near zone and $5 \%$ in the far zone. Furthermore, there is even a small probability that $D_{s}$ slightly increases, corresponding to a negative value of the delay spread reduction. This occurs in a zone of the tunnel where the number of multipath components is small, such as when $d=200 \mathrm{~m}$, as shown in Figure 4.29. This absence of reduction of $D_{s}$ with TR alone is due to the autocorrelation operation which nearly doubled the impulse response. With MISO TR and for a probability of 0.5, the delay spread reduction is $75 \%$ in the near zone but decreases to $20 \%$ at large distances from the transmitter.

For a simple receiver picking up the peak energy of the impulse response, another interesting metric is the focusing gain, also called the peak to peak gain, noted $G_{p 2 p}$ and defined as the ratio of the strongest tap power received with TR or without TR [Naqvi and El Zein, 2008], [Pajusco and Pagani, 2009]. It is thus given by:

$$
G_{p 2 p}\left(d_{0}\right)=\left(\frac{\max _{\tau}\left(\left|y_{T R}\left(d_{0}, \tau\right)\right|^{2}\right)}{\max _{\tau}\left(\left|y\left(d_{0}, \tau\right)\right|^{2}\right)}\right)
$$

where $y_{T R}\left(d_{0}, \tau\right)$ and $y\left(d_{0}, \tau\right)$ denotes the received signal amplitude using TR and not using TR, respectively.

The curves in Figure 4.31 represent the variation of $G_{p 2 p}$ versus distance, for TR and for $4 \times 1$ MISO TR, respectively. It should be noted that the gain with SISO TR is between 2 and $6 \mathrm{~dB}$, except in the zone situated at $200 \mathrm{~m}$ from the transmitter where the gain becomes negligible, as already outlined. When using MISO TR, $G_{p 2 p}$ is $6 \mathrm{~dB}$ better than for SISO TR, since all the energies from different elements coherently add up.

\subsection{Conclusions}

In this chapter, multidimensional propagation characteristics in an empty straight arc-shaped tunnel were deduced from measurements in the $2.8-5 \mathrm{GHz}$ UWB frequency band. On the one hand, some parameters that characterizes the propagation channel have been deduced from measurements. On the other hand, these parameters are also used to perform a multi-dimensional analysis (space, frequency, polarization and delay-space) of different diversity techniques proposed.

Firstly, some parameters that characterizes the propagation channel have been extracted for this particular environment. The guiding effect of the tunnel clearly appears on the mean path loss, the average attenuation between $50 \mathrm{~m}$ and $500 \mathrm{~m}$ being of about $4 \mathrm{~dB}$. The analysis of the correlation in the transverse plain shown that the channel is highly correlated for distances between Tx-Rx larger than $100 \mathrm{~m}$. Furthermore, the larger the antenna spacing, the lower the correlation coefficient is. 


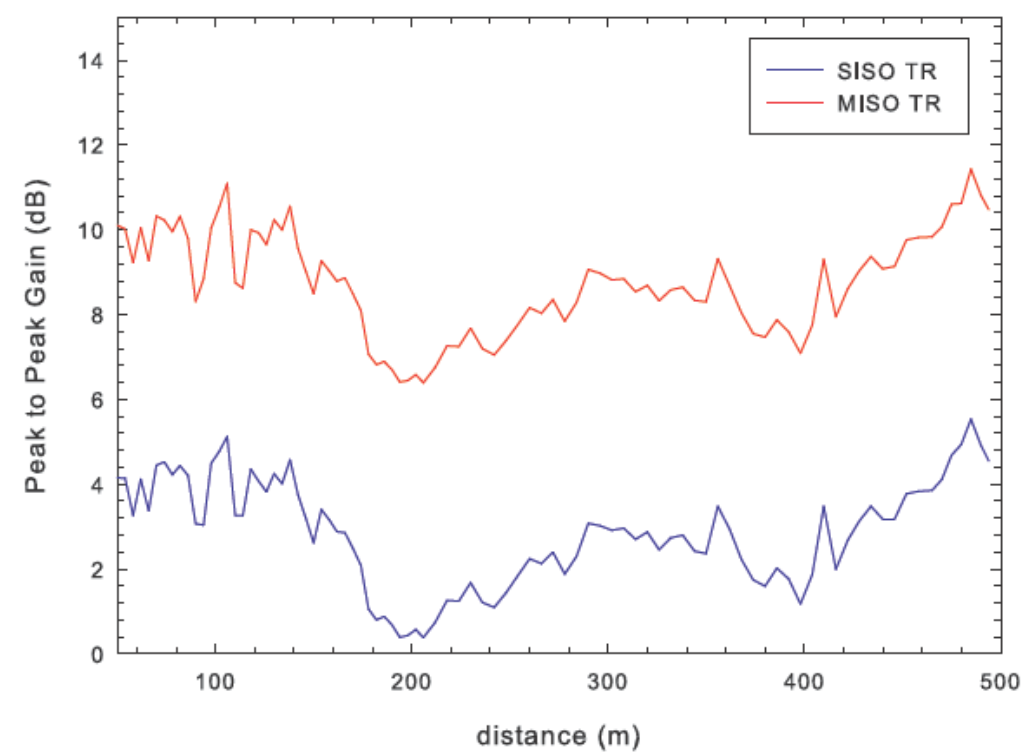

Figure 4.31. Peak to peak gain for SISO TR and MISO TR configurations.

The analysis of the double-directional propagation channel in tunnels has shown that the guiding feature of the tunnel was also clearly emphasized by the small values of the angular spread of the angle of departure/arrival, rapidly decreasing from $10^{\circ}$ at $50 \mathrm{~m}$ from the transmitter to $3^{\circ}$ at $200 \mathrm{~m}$. The theoretical results deduced from the ray theory assuming a rectangular tunnel slightly overestimate delay spread and angular spread, the curved shape of the tunnel ceiling being not taken into account, but the variations of these characteristic parameters with distance are quite similar.

Secondly, these parameters are used to evaluate the performance of some diversity techniques. Although in tunnels the correlation in both space and frequency increases with the distance between transmitter and receiver, the analysis of jointed space frequency diversity has shown a decrease of the correlation coefficient but only for short distances. This diversity is inefficient at large distance.

The influence of polarization (polarization diversity) in MIMO system capacity has been evaluated for a $4 \times 4$ configuration. Under the assumption of a constant SNR, array configurations made by a series of horizontally and vertically polarized elements (VHVH) or by 2 dual polarized elements outperform co-polarized arrays in terms of channel capacity. However, in the more realistic case of a constant transmitting power, the waves remain strongly polarized, especially at a large distance from the transmitter. It results in a decrease in the mean received power which balances the positive effect of a decrease in correlation. Thus an array architecture based on successive elements with different polarizations does not improve the channel capacity, keeping the length of the array constant. The most interesting configuration is to use dual-polarized elements, since it allows reducing the size of the array. 
Finally, the performance of Time Reversal technique applied to impulse radio UWB channels has been analyzed both for SISO and MISO configurations. It has been shown that SISO TR does not appreciably reduce the delay spread. In contrast, when the distance between the transmitter and the receiver does not exceed $250 \mathrm{~m}$, the delay spread with MISO TR is reduced from a few ns without TR to $1 \mathrm{~ns}$. The peak to peak power gain with SISO TR is $3 \mathrm{~dB}$ on average, except in a zone of the tunnel where the number of multipath components becomes small, this zone being situated at about 200 $\mathrm{m}$ from the transmitter in our tunnel configuration. If 4 antennas are used on the transmitting site, the peak to peak gain increases by a factor of 2 compared to SISO TR, as it would be expected from previous works on MISO TR, and reaches $10 \mathrm{~dB}$. Despite the guiding effect of the tunnel, leading to a propagation of the waves nearly along the tunnel axis, i.e. to rays reflecting on the tunnel walls with a grazing angle of incidence, MISO TR can be an interesting solution to improve the performance of the link. 
Chapter 5

\section{CONCLUSIONS AND PERSPECTIVES}

The design of future wireless communications strongly depends on the propagation channel. Furthermore, multiantenna techniques (MIMO, MISO, SIMO) have emerged as a good technique to optimize the use of the transmission spectrum and power as well as increase the data transfer rate.

The propagation channel can be modeled theoretically or experimentally. In contrast with theoretical characterization, experimental characterization provides a precise knowledge of the real scenario under analysis. However, experimental characterization requires not only undertake measurements in the desired environment, but also, develop adequate channel sounders.

This thesis has dealt with the experimental characterization of the propagation channel with large bandwidth and multiple antennas, by means of the analysis of measurements in different environments as well as the development and improvement of two channel sounders.

The main contributions of this thesis are presented in Section 5.1, while future research work is proposed in Section 5.2 . 


\subsection{Conclusions}

As mentioned before, this thesis is focused in the experimental characterization of the radio channel for multiantenna systems and for large bandwidth channels.

Chapter 2 dealt with the improvement of a frequency-domain MIMO channel sounder and the development of a time-domain MIMO channel sounder. Firstly, an existing frequency-domain channel sounder based on a multiport network analyzer was modified in order to accomplish high resolution measurements in the spatial domain. To accomplish this an XY positioning board and two stepper motors were used. It was shown how the RMS delay spread varied significantly from one position to another within an area of $60 \times 60 \mathrm{~cm}^{2}$. Furthermore, the complex receiving correlation was calculated in such a small area, showing that for NLoS situations, the correlation decreases more rapidly than when transmitter and receiver are in LoS. Secondly, the same channel sounder was also integrated in a geographical information system (GIS). Thus, measurements were georeferenced as well as the locations of both transmitter and receiver. This application makes the organization and storing of the measurements easier.

Finally, a $2 \times 2$ MIMO time-domain channel sounder based on the use of commercial and multipurpose equipment was developed. The main advantage of this kind of channel sounders was the low acquisition time compared to classical channel sounders based on a network analyzer. The channel sounder was made up with multipurpose and commercial equipment such as a base-band generator, an I/Q modulator, and an oscilloscope. Measurements in a corridor were undertaken showing that the channel response measured by the time-domain system and the frequency-domain system exhibited a similar behavior. Moreover, the RMS delay spread and correlation coefficients computed show similar values for both measurement systems.

In Chapter 3 , the results from an indoor $4 \times 4 \mathrm{MIMO}$ measurement campaign in the $2-5 \mathrm{GHz}$ UWB frequency band were reported. The path loss for VV and $\mathrm{HH}$ polarizations (co-polar) was analyzed by computing the mean path loss, while VH and $\mathrm{HV}$ polarizations (cross-polar) were analyzed by means of the analysis of the XPD. The path loss for co-polar configurations always increased linearly with distance. In the case of the laboratories, each wall (made of plasterboard) was modeled with an extra loss. In contrast, while the XPD in LoS in the corridor was found to be nearly constant with distance, it decreased with distance in NLoS for propagation in laboratories. Regarding delay dispersion, in the corridor scenario (LoS) the RMS delay spread was around 3 ns and was constant with distance, while in the laboratories it increased with the number of walls penetrated.

The same analysis was performed the in frequency domain, in $500 \mathrm{MHz}$ sliding-window intervals. The decay factor was found to be constant with frequency in all environments, as well as the losses of each wall of the laboratories. The central frequency did not influence the behavior of the XPD, which was also constant with frequency. Although the analysis in the frequency domain of 
delay dispersion implies an increment of the RMS delay spread caused by the narrower bandwidth used, it was shown that frequency did not have an effect on the delay dispersion.

Finally, the capacity of the $4 \times 4$ MIMO-UWB system was analyzed in these scenarios for different polarizations $(\mathrm{HH}, \mathrm{VV}, \mathrm{HV}$, and $\mathrm{VH}$ ). If considering constant $\mathrm{SNR}$, the capacity for the copolar configurations (VV and $\mathrm{HH}$ polarizations) in LoS conditions was found to be higher than for the cross-polar ones. However, the contrary behavior was found when the receiver was in NLoS with the transmitter. In the case of variable SNR, the capacity was strongly influenced by the SNR. Thus, the capacity tended to decrease as the SNR did in all the environments under analysis.

In this thesis, the particularities of propagation in tunnels have been studied in Chapter 4 To accomplish this, measurement in the $2.8-5 \mathrm{GHz}$ UWB frequency band in a empty tunnel were analyzed. The guiding effect of the tunnel was observed on the mean path loss while the RMS delay spread was observed to decrease with distance only at the beginning of the tunnel. The analysis of the correlation shown that in the transverse plane the received signals are rapidly uncorrelated for short distances and highly correlated for large distances.

The analysis of the double-directional propagation channel in tunnels shown that the guiding feature of the tunnel was also confirmed by the small values of the angular spread of the angle of departure/arrival. In frequency domain (fequency diversity) a higher decorrelation in frequency was found at short distances Tx-Rx, by using MIMO and increasing the separation in the spatial domain.

The performance of some diversity techniques were also studied for propagation in tunnels. The polarization diversity was evaluated for a $4 \times 4$ MIMO configuration. If considering a constant SNR, array configurations made by a series of horizontally and vertically polarized elements (VHVH) or by 2 dual polarized elements outperform co-polarized arrays in terms of channel capacity. However, for a constant transmitting power an array architecture based on successive elements with different polarizations does not improve the channel capacity, keeping the length of the array constant.

Finally, the performance of Time Reversal technique applied to impulse radio UWB channels was proposed for SISO and MISO configurations. For a SISO configuration, Time Reversal did not appreciably reduce the delay spread. However, for a MISO configuration the delay spread with Time Reversal was reduced considerably. The gain in terms of the peak received power increases considerably in the case of using Time Reversal with a MISO configuration. In summary, it was shown that MISO-Time Reversal can be an interesting solution to improve the performance of the radio link in tunnels. 


\subsection{Perspectives}

As in any research field, the development of this thesis has led to ideas which may contribute to the improvement and future extension of the work here presented. This section briefly specifies these future research lines.

One immediate extension of this work would be the measurement and analysis of the smallscale fading. To accomplish this, the high spatial resolution frequency-domain channel sounder developed in Chapter 2, Section 2.2.1, could be used due to its high resolution in the spatial domain. Furthermore, the double directional propagation channel for indoor environments could be analyzed with this system by using high resolution algorithms such as RIMAX or SAGE.

Regarding the time-domain channel sounder (Chapter 2, Section 2.3) one of the main improvements could stem from the improvement of the acquisition speed in the time-domain channel sounder proposed in Chapter 2. Although the acquisition time is very short, the time required for storing the waveforms in the scope hard disk is high (compared with the acquisition time). This time makes that the acquisition time between two channel impulse responses is not as low as desired. Another improvement could be the introduction of clock references to synchronize the transmitting and receiving side of this system without cables. This clock reference should work slower than the repetition frequency of the code generator. With this improvements, time-variant channels could be measured with this channel sounder more accurately.

In Chapter 3 the MIMO-UWB radio channel was analyzed for different polarization. In this field, a future research line could be the comparison of the results with those provided by a ray tracing software in order to confirm the propagation path loss models proposed.

Despite of the fact that many papers in literature address the problem of polarization, there is not too much information about the analysis of the XPD. Thus, an extension of this work could be the analysis of the relation between the XPD and other parameters that characterizes the propagation channel such as the RMS delay spread.

In Chapter 4 measurements in a empty tunnel were carried out. This measurements were performed for several polarizations. Another possible extension of this work could be the modeling of the XPD in tunnels and the comparison with results obtained in Chapter 3 for an indoor scenario. Furthermore, time-variant measurements in tunnels could be performed with the new time-domain channel sounder. Thus, the Time Reversal technique could be evaluated not only in static conditions but also for channels that vary with time. 
Appendices 



\section{Appendix A}

\section{Multiple-INPUT}

\section{Multiple-OutPut (MiMO)}

MIMO systems emerged few years ago as a technique for optimizing the use of the spectrum as well as increasing the transmitting data rates. This appendix is devoted to review the most important aspects of MIMO systems which are directly related with this thesis.

In Section A.1, the multiantenna configurations as well as the MIMO system capacity are addressed. Section A.2 presents the main benefits offered by MIMO depending on the propagation channel. Section A.3 describes the relation between correlation and capacity. Section A.4 outlines the difference between outage and ergodic capacity.

\section{A.1. Multiantenna Systems}

Traditional wireless communications systems are those with one antenna at the transmitter and one antenna at the receiver (Single-Input Single-Output, SISO). Shannon [Shannon, 1948] predicted the maximum theoretical capacity that can be achieved using this kind of systems as:

$$
C(\text { bps } / H z)=\log _{2}\left(1+\rho|h(t)|^{2}\right)
$$


where $\rho$ is the Signal-to-Noise ratio (SNR) at the receiver and $h(t)$ is the normalized channel impulse response. This capacity can be modified by increasing the number of transmitters or receivers SIMO, MISO) in order to provide transmitting or receiving diversity respectively, but it does not increase the channel capacity.

At the same time, the increment of the demand of the spectrum has motivated the research of new techniques that improve the use of this spectrum. One of these techniques is the use of multiple antennas at both sides of the radio channel (Multiple-Input Multiple-Output, MIMO) [Foschini and Gans, 1998], [Telatar, 1995]. All these configurations are shown in Figure A.1.

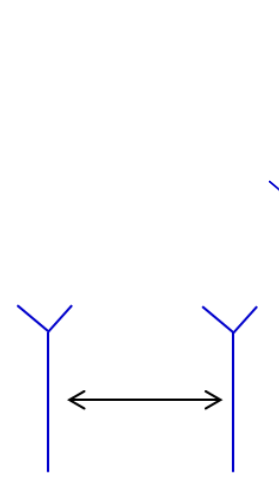

(a)

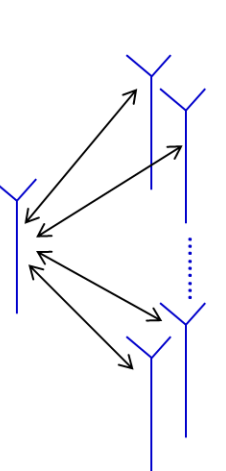

(b)

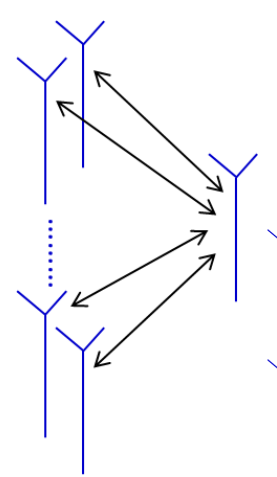

(c)

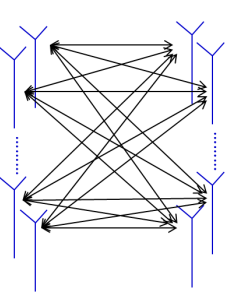

(d)

Figure A.1. Multiantenna configurations: (a) SISO, (b) SIMO, (c) MISO and (d) MIMO.

It has been demonstrated that the use of multiple antennas at both sides of the radio channel, increase the number of transmitted bits per hertz. This enhancement of the capacity depends directly on the dispersion of the environment and it allows that the transmitted information can be sent by independent paths. For a MIMO system with $M$ transmitting antennas and $N$ receiving antennas, the capacity is computed as:

$$
C(b p s / H z)=\log _{2}\left(\operatorname{det}\left(I_{N}+\frac{\rho}{M} H(t) H^{\dagger}(t)\right)\right)
$$

where $\boldsymbol{H}$ is the normalized MIMO transfer matrix of dimensions $N \times M$ and ()$^{+}$denotes transpose conjugated. The maximum capacity that can be achieved is $\min \{N, M\}$. The MIMO transfer matrix consist of $N \times M$ transfer functions $h_{i j}$ when the antenna $j$ is transmitting and the antenna $i$ is receiving:

$$
\boldsymbol{H}(\tau)=\left[\begin{array}{cccc}
h_{11}(\tau) & h_{12}(\tau) & \cdots & h_{1 M}(\tau) \\
h_{12}(\tau) & h_{22}(\tau) & \cdots & h_{2 M}(\tau) \\
\vdots & \vdots & \ddots & \vdots \\
h_{N 1}(\tau) & h_{N 2}(\tau) & \cdots & h_{N M}(\tau)
\end{array}\right]
$$


This matrix can be characterized by its eigenvalues $\lambda_{i}$, which represent one independent subchanel in the MIMO channel. In this case, the MIMO capacity can be computed as:

$$
C(b p s / H z)=\sum_{i=1}^{K} \log _{2}\left(1+\frac{\rho}{N} \lambda_{i}\right)
$$

\section{A.2. MIMO Benefits}

Depending on the MIMO propagation channel, MIMO systems can offer three main different benefits (Figure A.2), as:

Beamforming Gain - the transmit and receive antenna patterns can be focused into a specific angular direction by the appropriate choice of complex baseband antenna weights. The more correlated the antenna signals, the better for beamforming. Under LoS conditions, the receiver and transmitter gains may add up, leading to an upper limit of $N \times M$ for the beamforming gain.

Spatial Diversity - multiple replicas of the radio signal from different directions in space give rise to spatial diversity, which can be used to increase the transmission reliability of the fading radio link. For a spatially white MIMO channel, that is, completely uncorrelated antenna signals, the diversity order is limited to $N \times M$. Spatial correlation will reduce the diversity order and is therefore an important channel characteristic.

Spatial Multiplexing - MIMO channels can support parallel data streams by transmitting and receiving or orthogonal spatial channels. The number of usefully multiplexed streams depends on the spatial properties of the radio environment. The spatial multiplexing gain may reach $\min \{N, M\}$ in a sufficiently rich environment.

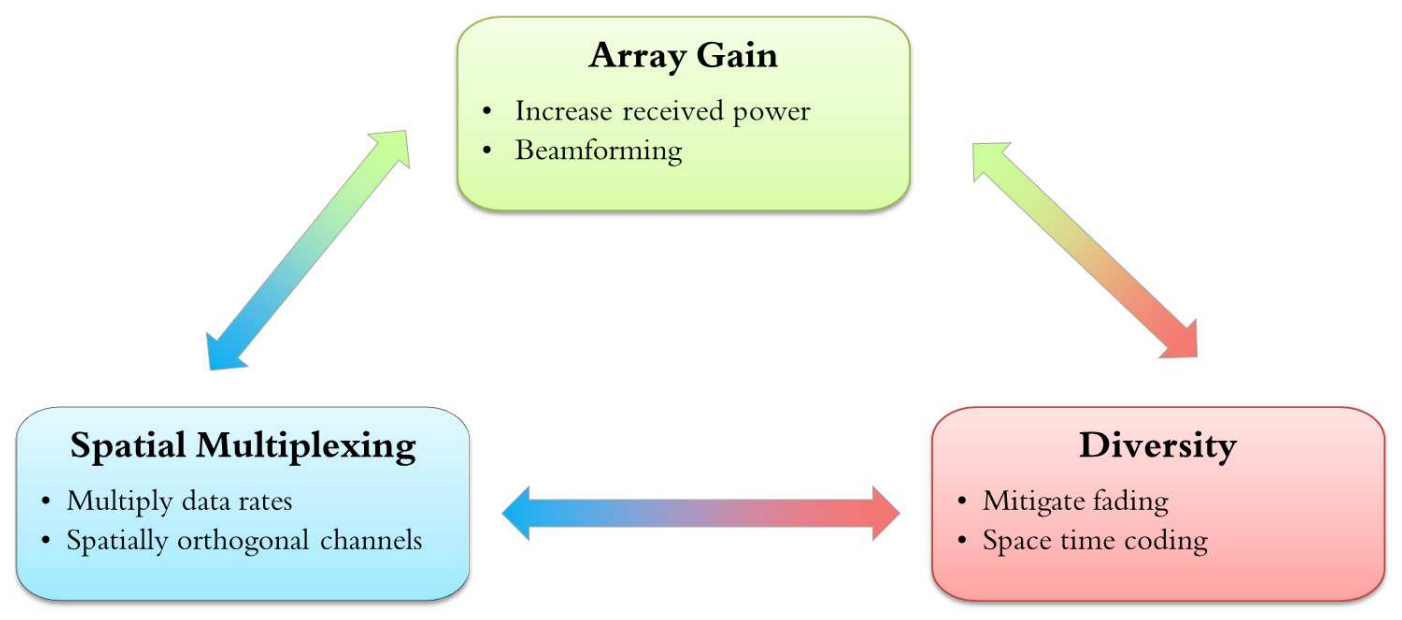

Figure A.2. Benefits that MIMO can offer. 
Beamforming, diversity and multiplexing are rivaling techniques. To highlight the role of the propagation channel, the threefold trade-off between beamforming, diversity and multiplexing can be broken dowin into several sub-tradeoffs [Sibille et al., 2011]. The partial overlap of the ellipses in Figure A.3 indicates that there is a gradual transition between the pure realizations of a certain MIMO benefit. In summary, it is the propagation environment that determines what can be gained by MIMO techniques.

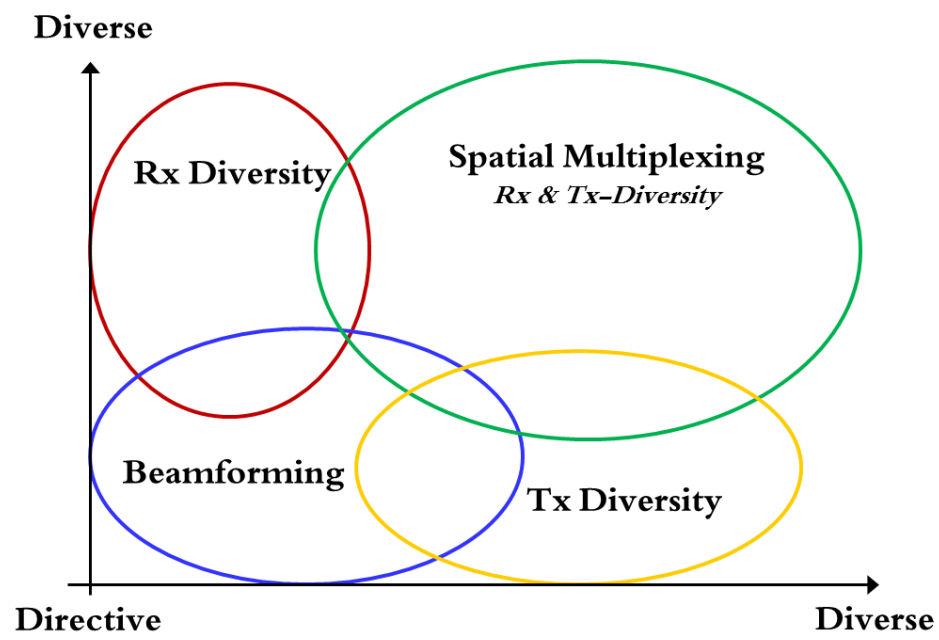

Figure A.3. Directivity or diversity at the receiving and transmitting arrays determine if the channel supports beamforming, diversity, or spatial multiplexing.

\section{A.3. Correlation}

The success of MIMO systems depends on there being a low correlation between the different subchannels defined between the transmitter and receiver array elements [Fernandez et al., 2005]. The topology of the elements of the array, the distance between elements or the multipath signal can reduce the correlation and therefore, the number of effective subchannels is reduced and thus the capacity of the system is reduced too [Shannon, 1948].

The correlation of the MIMO system is characterized by means of the correlation matrix $R$, composed by the correlation coefficients between two elements of the transmitting or receiving array. Therefore, the complex correlation coefficient at the frequency $f$ for the $k t h$ time-realization between the reception antennas $p$ and $q$ can be computed as:

$$
\rho^{p, q}(f, k)=\frac{E\left[\overline{h^{p}(f, k)} \cdot \overline{h^{q}(f, k)}\right]}{\sqrt{E\left[\left.\overline{h^{p}(f, k)}\right|^{2} \cdot\left|\overline{h^{q}(f, k)}\right|^{2}\right]}}
$$

where $E[]$ is the expectation operation value for all the transmitting element antennas, and $\overline{h^{p}(f, k)}$ is the zero mean $p$ th row of the $\boldsymbol{H}$ matrix. 
The correlation coefficient between the transmitting antennas can be calculated in a similar way by swapping the rows and the columns on the matrix.

\section{A.4. Ergodic Capacity and Outage}

As Shannon proposed [Shannon, 1948], the capacity for a user in a time-invariant channel is the maximum information between transmitter and receiver. It can be also defined as the maximum information transfer rate that can be transmitted by the radio channel with an arbitrary probability error.

If the channel is random and variant, the capacity becomes a random variable. In this case, the Ergodic Capacity as well as the Outage Capacity are used to measure the capacity of the system. On the one hand, the Ergodic Capacity is the maximum mutual information transfer averaged between all the channel states.

On the other hand, the Outage Capacity outlines the percentage of time in which capacity is higher than a certain value. In this way, a outage capacity $C_{0.01}=10 \mathrm{bits} / \mathrm{s} / \mathrm{Hz}$ shows that the $99 \%$ of time, the capacity is higher than 10bits/s/ Hz. 


\section{Appendix B}

\section{CHARACTERIZATION OF THE Wideband Radio Propagation CHANNEL}

The mobile radio propagation environment places fundamental limitations on the performance of radio communication systems. Signals arrive at a receiver via several propagation mechanisms: reflection, diffraction or scattering. These phenomena give rise to receive multiple replicas of the transmitted signals due to the existence of multiple propagation paths (from now on, multipath propagation). Thus, the received signals have different time delays, attenuations and phases and therefore, the transmission channel results highly complex.

The characterization of the propagation channel is a crucial issue to accurate design and develop wireless devices. Therefore, in the following sections the main aspects that characterize the radio propagation channel are reviewed.

Firstly, Section B.1 and Section B.2 address the difference between large and small scale fading respectively. Secondly, Section B.3 presents the four functions that characterizes deterministic and random radio channels. Next, in Section B.4 stochastic channels are classified depending on some practical constrains. Finally, Section B.5 presents the main parameters that characterize the mobile 
radio channel such as the path loss, the RMS delay spread, the coherence bandwidth or the angular spread. Such parameters are widely use in this thesis.

\section{B.1. Large-Scale Fading}

As a mobile moves over very small distances, the instantaneous received power may fluctuate rapidly giving rise to small-scale fading. The reason for this is that the received signal is a sum of many contributions coming from different directions, as mentioned previously. Since the phases are random, the sum of the contributions can vary considerably. In small-scale fading, the received signal power may vary up to three or four orders of magnitude (30 or $40 \mathrm{~dB}$ ) when the receiver is moved by only a fraction of a wavelength. As the mobile moves away from the transmitter over much larger distances, the local average received signal will gradually decrease, which is called thelarge-scale fading. Typically, the local average received power is computed by averaging signal measurements over a measurement track of $5 \lambda$ to $40 \lambda$ [Rappaport, 1996].

\section{B.2. Small-Scale Fading}

The small-scale fading describes the rapid fluctuations of the radio propagation channel as a consequence of multipath propagation over a short period of time or travel distance, so that largescale effects may be ignored. Small-scale fading is caused by interference between two or more replicas of the transmitted signal which arrive at the receiver at slightly different times. These waves combine at the receiver antenna to give a resultant signal which can vary widely in amplitude and phase, depending on the distribution of the intensity and relative propagation time of the waves and the bandwidth of the transmitted signal.

The small-scale fading can cause some effects such as [Rappaport, 1996] rapid variations in the power of the received signal over a small area or time interval, random frequency modulation as a consequence of varying Doppler shifts on different multipath signals, or delay dispersion due to multipath propagation delays.

The main physical phenomena that may influence small-scale fading are the multipath propagation, the movement of the mobile or the surrounding objects and the transmission bandwith of the signal. These are outlined below:

Multipath propagation - The presence of reflecting objects and scatterers in the channel creates a constantly changing environment that dissipates the signal energy in amplitude, phase, and time. These effects result in multiple replicas of the transmitted signal that arrive at the receiving antenna, shifted with respect to one another in time and spatial orientation. 
Speed of the mobile - The relative motion between the transmitter and receiver results in random frequency modulation due to different Doppler shifts on each of the multipath components. Doppler shift will be positive or negative depending on whether the mobile receiver is moving toward or away from the base station.

Speed of surrounding objects - If objects in the radio channel are in motion, they induce a time varying Doppler shift on multipath components. If the surrounding objects move at a greater rate than the mobile, then this effect dominates the small-scale fading. Otherwise, motion of surrounding objects may be ignored, and only the speed of the mobile need be considered.

The transmission bandwidth of the signal - If the transmitted signal bandwidth is greater than the bandwidth of the multipath channel, the received signal will be distorted, although the received signal power will not vary much over a local area (i.e., the small-scale signal fading will not be significant). As is explained in Section B.5.3, the bandwidth of the channel can be quantified by the coherence bandwidth, which is a measure of the maximum frequency difference for which signals are still strongly correlated in amplitude.

\section{B.3. Characterization of Deterministic and Random Channels}

The radio propagation channel can be considered as a system which transforms input signals into output signals in the same way as a time-varying linear filter. Therefore, the inputs and outputs of the channel can be described in both time and frequency domains [Parsons, 2000]. Thus, four transmission functions describe the channel as explained next.

The time domain function - As any linear system, the time domain domain description of the propagation channel is described by its time impulse response. Since the channel can be timevariant, its impulse response is also a time-varying function. Taking into account that, in general, real passband systems are described by their complex envelopes (equivalent low pass), the channel impulse response $h(t, \tau)$ consists of a series of attenuated, time delayed, phase shifted replicas of the transmitted signals as [Rappaport, 1996]:

$$
h(t, \tau)=\sum_{i=0}^{N-1} a_{i}(t, \tau) \exp \left[j\left(2 \pi f_{c} \tau_{i}(t)+\phi_{i}(t, \tau)\right)\right] \delta\left(\tau-\tau_{i}(t)\right)
$$

This function was also called by Bello [Bello, 1963] the input delay-spread function.

The frequency domain function - The channel function $H(f, v)$ relates the channel output spectrum to the channel input spectrum in the same way as $h(t, \tau)$ relates the input/output time functions. It was also termed by Bello [Bello, 1963] as the output Doppler-spread function.

The time-variant transfer function - This function of the channel, $T(f, \tau)$, relates the output time 
function with the input spectrum, that is, describes the frequency transfer function as a function of time.

The delay/Doppler spread function - The information about time-delay and Doppler-shift domains is combined in the delay Doppler spread function $S(\tau, v)$. This function describes the dispersive behaviour of the channel in terms of both time delays and Doppler shifts.

These four bidimensional functions are related via the Fourier transform $\mathfrak{F}$ or the inverse Fourier transform $\mathfrak{F}^{-1}$ as it is depicted in Figure B.1.

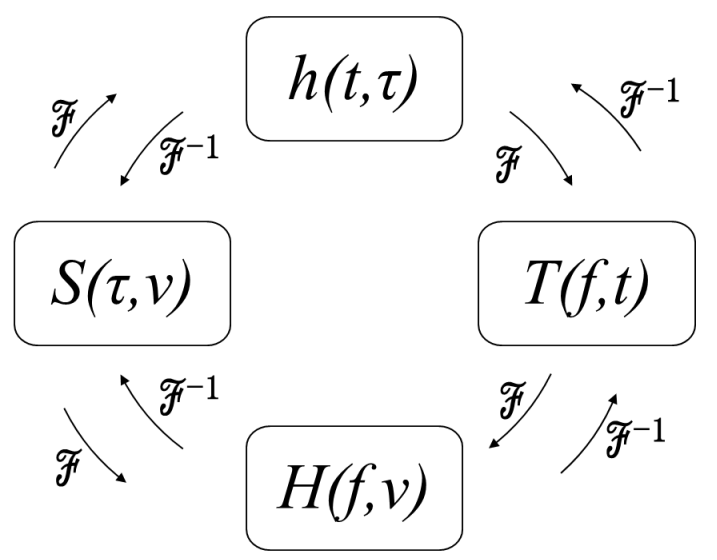

Figure B.1. Relationships between system functions.

This analysis for deterministic channels can be extended to real radio channels. In this case, the channel vary in time randomly and the system functions explained before become stochastic processes. The simplest approach to analyze these functions is based on obtaining the correlation functions of the system functions [Bello, 1963]. Under these assumptions:

- $h(t, \tau)$ becomes $R_{h}(t, s ; \tau, \eta)$

- $H(f, v)$ becomes $R_{H}(f, m ; v, \mu)$

- $T(f, t)$ becomes $R_{T}(f, m ; t, s)$

- $S(\tau, v)$ becomes $R_{S}(\tau, \eta ; v, \mu)$

where $t$ and $s$ are time variables, $\tau$ and $\eta$ are delay variables, $f$ and $m$ are frequency variables and $v$ and $\mu$ are Doppler frequency-shift variables.

\section{B.4. Classification of Practical Channels}

As mentioned in Section B.3, real radio channel are stochastic processes which are characterized by four correlation functions. These channel functions can be simplified considering several 
constrains given rise to the following classification of channels:

Wide-sense Stationary Channel (WSS) - These channels have the property that the channel correlation functions and therefore, the fading statistics, are invariant over a short period of time $\xi$. Thus, WSS channels give rise to uncorrelated Doppler-shift scattering.

Uncorrelated Scattering Channel (US) - In this case, the contributions from elemental scatters with different path delays are uncorrelated.

Wide-sense Stationary Uncorrelated Scattering Channel (WSSUS) - These channels combines the properties of the WSS and US channels and fortunately, most of the radio channels can be assumed to be WSSUS. This kind of channels exhibits uncorrelated dispersiveness in both the time-delay (US) and Doppler-shift (WSS) domains.

From now on, the radio propagation channel will be assumed to be WSSUS. Thus, some parameters that characterize the radio channel will be described in the next section.

\section{B.5. Parameters of Mobile Multipath Channels}

Many channel parameters are derived from the Power Delay Profile, (PDP), which is computed by averaging spatially the channel impulse response, $|h(t, \tau)|^{2}$, over a local area [Rappaport, 1996] and represents the power as a function of time, $t$, and delay $\tau$. Considering a certain snapshot, $t=t_{0}$, the $P D P$ becomes a single time-invariant power delay profile, $P(\tau)$.

\section{B.5.1. Path Loss and Shadowing}

The path loss can be considered to be one of the most important parameters which characterize the propagation channel and influences strongly the performances of a wireless communications system. It is defined as the ratio between the transmitted power and the received power, this is, the attenuation of the transmitted signal in the radio link.

In a wideband system, where the bandwidth of the transmitted signal is higher than the coherence bandwidth (see Section B.5.3), the path loss can be deduced from the channel impulse response as:

$$
P L=\frac{1}{\int_{\tau}|h(t, \tau)|^{2} d \tau}
$$

Commonly, this parameter is expressed in logarithmic units as:

$$
P L(d B)=-10 \log \left(\int_{\tau}|h(t, \tau)|^{2} d \tau\right)
$$


Both theoretical and measurement-based propagation models indicate that average received signal power decreases logarithmically with distance, whether in outdoor or indoor radio channels [Rappaport, 1996]. The average large-scale path loss for an arbitrary distance between transmitter and receiver, $d$, is expressed as a function of distance by using a path loss exponent, $n$ as:

$$
P L(d B)=P L\left(d_{0}\right)+10 \mathrm{n} \log \left(\frac{d}{d_{0}}\right)
$$

where $n$ is the path loss exponent which indicates the rate at which the path loss increases with distance and deppends on the environment. $P L\left(d_{0}\right)$ is the path loss at a reference distance (usually 1 m.).

Nevertheless, even for the same distance between transmitter and receiver, surrounding elements can vary dramatically the path loss for two different locations in similar environments. This phenomenon is called log-normal shadowing. In this case, the path loss at a particular location can be defined as a random and distributed log-normally (normal in $\mathrm{dB}$ ) about the mean path loss. Thus, the path loss can be described by:

$$
P L(d B)=P L\left(d_{0}\right)+10 \mathrm{n} \log \left(\frac{d}{d_{0}}\right)+X_{\sigma}
$$

where $X_{\sigma}$, is a zero-mean Gaussian distributed random variable (in $\mathrm{dB}$ ) with standard deviation $\sigma$ (also in $\mathrm{dB})$.

\section{B.5.2. Delay Dispersion Parameters}

The time dispersive properties of wide band multipath channels are commonly quantified by their mean excess delay, $\bar{\tau}$, and RMS (Root Mean Square) delay spread, $\sigma_{\tau}$. The mean excess delay is the first moment of the power delay profile, $P D P$, and is defined as [Rappaport, 1996]:

$$
\bar{\tau}=\frac{\sum_{k} P\left(\tau_{k}\right) \tau_{k}}{\sum_{k} P\left(\tau_{k}\right)}
$$

The RMS delay spread is the square root of the second central moment of the PDP as:

$$
\sigma_{\tau}=\sqrt{\overline{\tau^{2}}-(\bar{\tau})^{2}}
$$

where

$$
\overline{\tau^{2}}=\frac{\sum_{k} P\left(\tau_{k}\right) \tau_{k}^{2}}{\sum_{k} P\left(\tau_{k}\right)}
$$


Thus, the rms delay spread can be computed as:

$$
\sigma_{\tau}=\sqrt{\frac{\sum_{k} P\left(\tau_{k}\right) \tau_{k}^{2}}{\sum_{k} P\left(\tau_{k}\right)}-\left(\frac{\sum_{k} P\left(\tau_{k}\right) \tau_{k}}{\sum_{k} P\left(\tau_{k}\right)}\right)^{2}}
$$

In practice, values for $\bar{\sigma}, \overline{\sigma^{2}}$, and $\sigma_{\tau}$ depend on the choice of noise threshold (in dB generally) used to process the PDP. The noise threshold is used to differentiate between received multipath components and thermal noise. If the noise threshold is set too low, then noise will be processed as multipath, thus giving rise to values of $\bar{\sigma}, \overline{\sigma^{2}}$, and $\sigma_{\tau}$ that are artificially high. Typical values are between $10-25 \mathrm{~dB}$ below the strongest path.

\section{B.5.3. Coherence Bandwidth}

Analogous to the delay spread parameters in the time domain, the coherence bandwidth, $B_{c}$, is used to characterize the channel in the frequency domain. Coherence bandwidth is a statistical measure of the range of frequencies over which the channel can be considered to have components with approximately equal gain and linear phase. In other words, coherence bandwidth is the range of frequencies over which two frequency components have a strong potential for amplitude correlation [Rappaport, 1996]. Thus, the coherence bandwidth can be deduced from the $-3 d B$ bandwidth (0.5 in linear units) of the autocorrelation of the frequency domain function $H(f)$.

The coherence bandwidth is related with the RMS delay spread as [Rappaport, 1996]:

$$
B_{c}=\frac{1}{5 \sigma_{\tau}}
$$

However, if the threshold used to deduce the coherence bandwidth is 0.9 , the relation between both parameters is:

$$
B_{c}=\frac{1}{50 \sigma_{\tau}}
$$

\section{B.5.4. Doppler Spread and Coherence Time}

Delay spread and coherence bandwidth are parameters which describe the delay dispersive nature of the channel in a local area. However, they do not offer information about the time varying nature of the channel caused by either relative motion between the mobile and base station, or by movement of objects in the channel.

Lets us assume a mobile receiver moving at a constant speed $v$ along a distance $d$. Due to its 
speed, the received signal experiences a phase change and therefore a frequency change also called Doppler shift, $f_{d}$ as [Rappaport, 1996]:

$$
f_{d}=\frac{v}{\lambda} \cos \theta
$$

where $\theta$ is the angle between the direction of motion of the mobile and direction of arrival of the waves.

Doppler spread and coherence time are two parameters which describe the time varying characteristics of the channel in a small-scale region due to the movement. When a mobile radio channel changes with a certain time rate, a spectral broadening is produced. Doppler spread $B_{D}$ is defined as the range of frequencies over which the received Doppler spectrum is essentially nonzero. When a pure sinusoidal tone of frequency is transmitted, the received signal spectrum, called the Doppler spectrum, will have components in the range $f_{c} \pm f_{d}$ were $f_{c}$ is the central frequency. The amount of spectral broadening depends on which is a function of the relative velocity of the mobile, and the angle $\theta$. It should be remarked that if the baseband signal bandwidth is much greater than $B_{D}$, the effects of Doppler spread are negligible at the receiver.

The coherence time is inversely proportional to the Doppler spread and is a statistical measure of the time duration over which the channel impulse response is invariant. This is, quantifies the similarity of the channel response at different times. The coherence time is the time duration over which two received signals strongly correlated in amplitude and can be computed as the time over which the time correlation function is above $-3 \mathrm{~dB}$.

\section{B.5.5. Angular Spread}

In MIMO systems it is possible to determine the direction of departure $D o D$ or $A o D$, and direction of arrival $D o A$ or $A o A$ of the waves. In this case, it can be useful to determine the spread of these angles as is analyzed in the delay domain with the RMS delay spread (see Section B.5.2). If the power delay profile as a function of delay and angle is available, $\operatorname{PDP}(\tau, \phi)$, the RMS angular spread can be computed as:

$$
\sigma_{\phi}=\sqrt{\frac{\sum_{k} P\left(\phi_{k}\right) \phi_{k}^{2}}{\sum_{k} P\left(\phi_{k}\right)}-\left(\frac{\sum_{k} P\left(\phi_{k}\right) \phi_{k}}{\sum_{k} P\left(\phi_{k}\right)}\right)^{2}}
$$




\section{Appendix C}

\section{FREQUENCY-DOMAIN CHANNEL SOUNDER}

As mentioned in this thesis, the knowledge of the MIMO propagation channel is a crucial issue when designing and implementing MIMO systems. One of the best techniques to characterize the MIMO channel is by means of experimental measurements in different environments. To accomplish this, the use of an adequate MIMO channel sounder it is necessary.

The work presented in Chapter 2 and Chapter 3 profits from a MIMO frequency-domain channel sounder existing in the Research Group of Mobile Communications (SiCoMo) [Ibernón-Fernández et al., 2008]. In this appendix this channel sounder is explained in detail.

Firstly, Section C.1 details both the hardware and software that compose the channel sounder. Section C.1.1 details the hardware that compose the channel sounder, while the software that controls the measurement routine as well as the measurement procedure is explained in Section C.1.2.

Finally, the measurement process is detailed in Section C.2. The first step is the calibration of the channel sounder which is described in Section C.2.1. Next, the measurement routine is detailed in Section C.2.2. 


\section{C.1. System Description}

As shown in Figure C.1, this channel sounder is based on a multiport vector network analyzer (MVNA) and a fast switch. The receiving antennas are directly connected to the ports of the MVNA. One port of the MVNA is configured as a transmitter and connected to an optical link (RF/OF and $\mathrm{OF} / \mathrm{RF}$ ), which carries the signal to the fast switch. Finally, the transmitting antennas are connected to the fast switch, so that the signal from the optical link is transmitted sequentially to each element of the transmitter array. The measurement process is controlled by a developed program executed on a laptop, which is connected to the MVNA by a LAN (Local Area Network) or Wiress LAN (WLAN) and to the fast switch by a GPIB (General-Purpose Interface Bus) port.

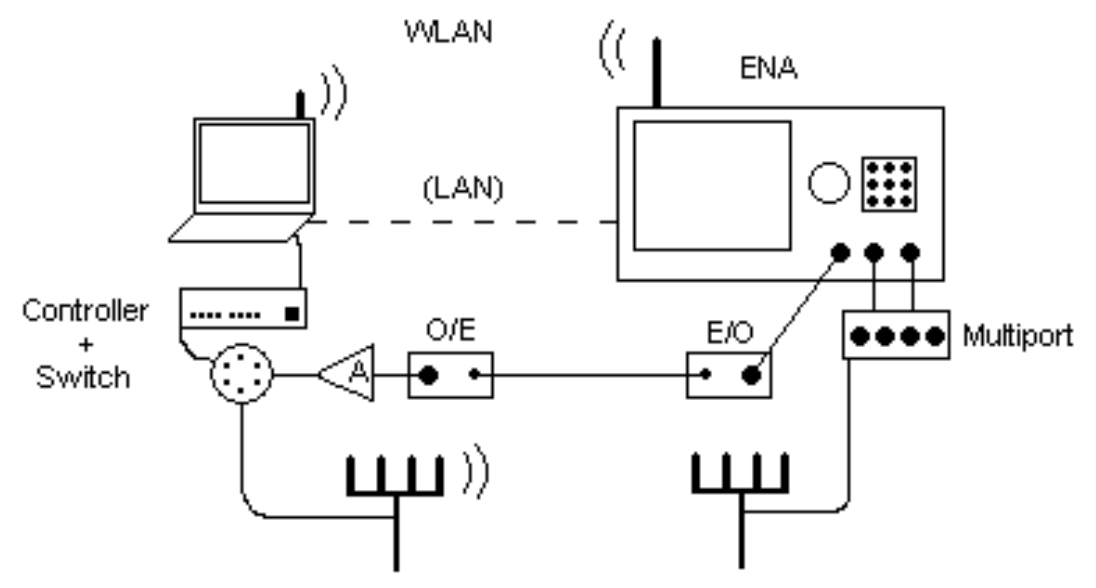

Figure C.1. Block diagram of the frequency domain channel sounder.

In the following sections both the hardware and the software used in this channel sounder are explained.

\section{C.1.1. Hardware}

This section is focused on the devices that compose the MIMO frequency-domain channel sounder: the MVNA, the switch and controller, the optical link, the computer and some other elements. In the following sections all these devices and the interconnection between them are detailed.

\section{C.1.1.1. Multiport Vector Network Analyzer}

The main element of this chanel sounder is a multiport vector network analyzer (MVNA), which is composed of the network analyzer Agilent ENA E5071B, and the Agilent Multiport Test Set E5091A as shown in Figure C.2. The MVNA can analyze signals from $300 \mathrm{kHz}$ to $8.5 \mathrm{GHz}$ using up to 1601 frequency points. 


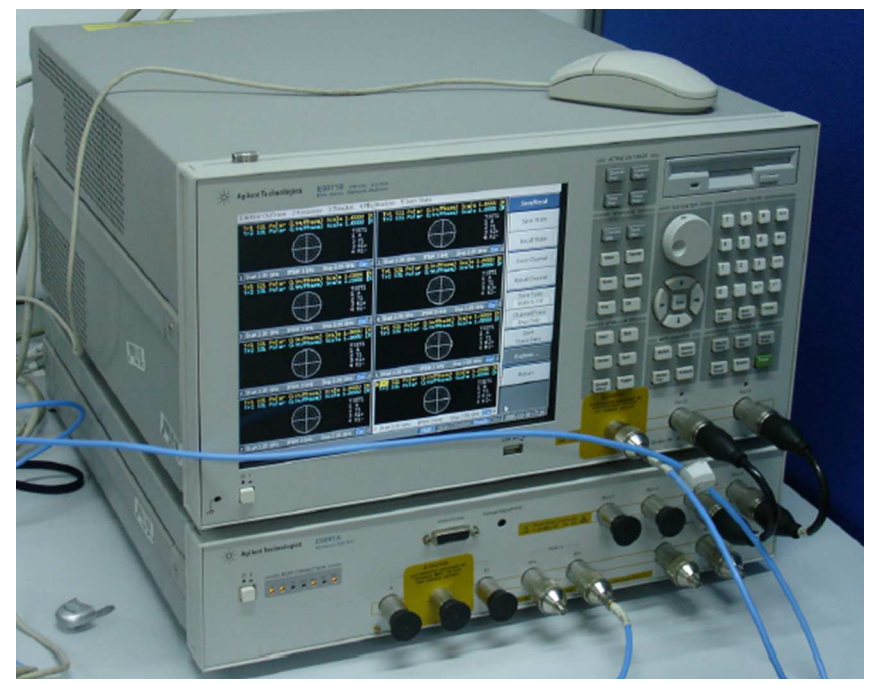

Figure C.2. Agilent Multiport Vector Network Analyzer.

As depicted in Figure C.1, the receiving antenna elements are directly connected to the MVNA while the transmitting elements are connected via an optical link to the fast switch as explained in sections Section C.1.1.2 and Section C.1.1.3.

\section{C.1.1.2. Switch and Controller}

A high-speed switch (Agilent 87406B) controlled by a switch driver (Agilent 11713A) has been used in order to provide the system with multiple transmitting antenna elements. In Figure C.3, the switch is mounted in a wooden box, and the controller is underneath. The port used in the MVNA as transmitter is connected to the high-speed switch (switch time up to 15ms) using a fiber optical link (See Section C.1.1.3). In our system, a six-port switch has been used, so up to 5 transmitting antenna elements can be used (multiplexer mode 1-5). This number could be incremented by using more than one switch. The signal coming out from the MVNA is switched into the different transmitting antenna elements (four blue cables in Figure C.3).

\section{C.1.1.3. Fiber Optic Link}

As mentioned in previous sections, the transmitting signal is carried to the switch via an optical link. To accomplish this, the optical transmitter (RF/OF) Ortel 3540A and the optical receiver (OF/RF) Ortel 4510B have been used. Their insertion losses are about $20 \mathrm{~dB}$. Thus, as shown in Figure C.1 the RF/OF device connects the MVNA with the fiber optic link, and the OF/RF connects the fiber optic link and the switch previously explained. The fiber optic link has a length of 50 meters (maximum distance between transmitter and receiver in this channel sounder) and an attenuation about $0.4 \mathrm{~dB} / \mathrm{km}$. 


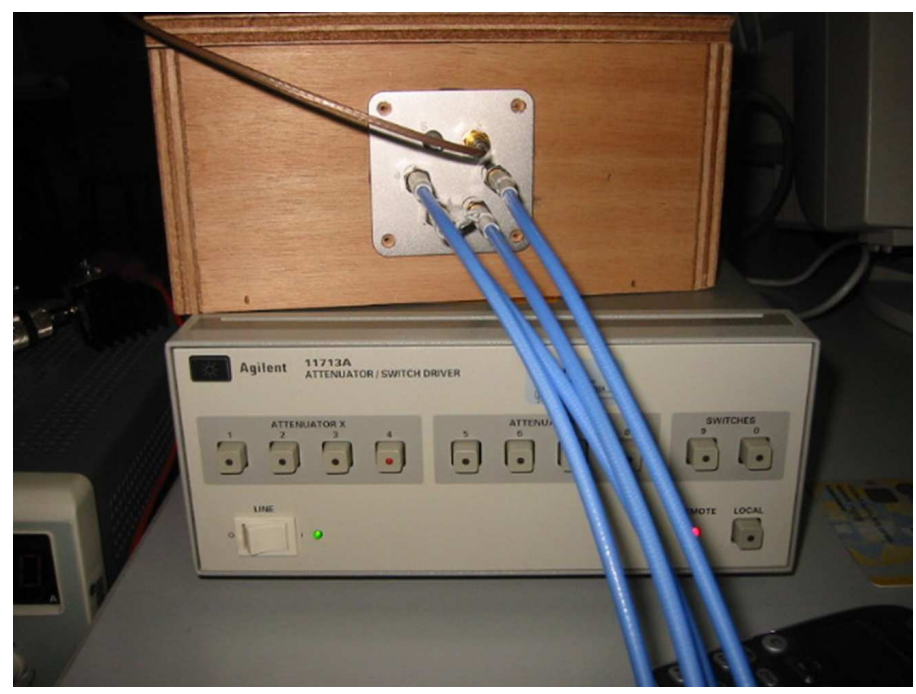

Figure C.3. Agilent Switch and controller.

\section{C.1.1.4. Computer}

A software application (see Section C.1.2), that controls all the elements of the system and guides the measurement routine, is execute in a PC. This PC is placed close to the switch/controller. The MVNA is controlled via LAN (Local Area Network) connection, and the controller via GPIB (General-Purpose Interface Bus) connection by using the Agilent 8235A USB/GPIB interface. The measurements are stored in the MVNA so as not to overload the LAN.

\section{C.1.1.5. Others}

Some other elements are used in the frequency-domain channel sounder depending on the application or the scenario to be measured.

The antennas used to sound the channel are selected depending of many factor such as the bandwidth and the central frequency under analysis, the gain or the dimensions.

In case of a large the path loss, some amplifiers can be installed in transmission and / or reception. Usually, the MiniCirtuits ZVE-8G+ Medium High Power (works in 2-8 GHz) are used. In this case, one power supply per amplifier is also necessary.

\section{C.1.2. Software}

The measurement procedure is controlled by an application developed in Visual Basic 6. This application configures the equipment with the measurement parameters as well as controls the measurement procedure. The main interface of this program is shown in Figure C.4. 


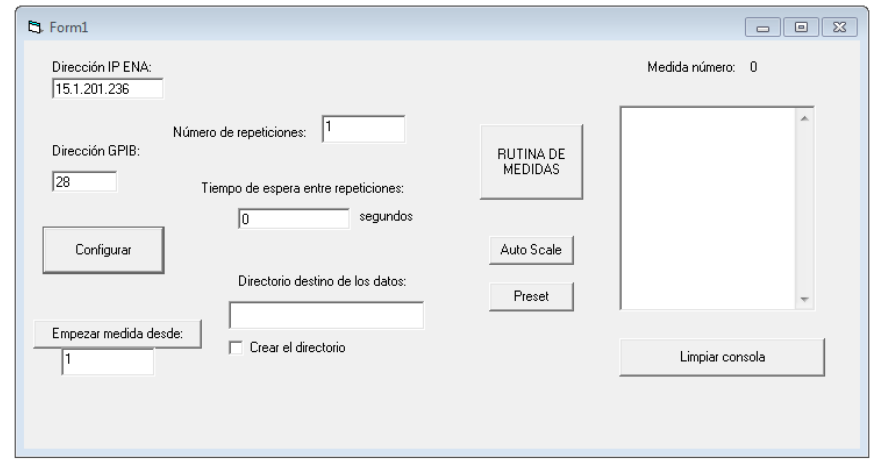

Figure C.4. Main interface of the software application.

The main parameters that the user should introduce in the software (see Figure C.5) are the the number of transmitting and receiving ( $\mathrm{M}$ and $\mathrm{N}$ ) elements, the initial and final frequency of the frequency band under analysis, the number of points. Other parameters are the IF (Intermediate Frequency) bandwidth, which controls the noise floor, and the measurements' format (by default polar format).

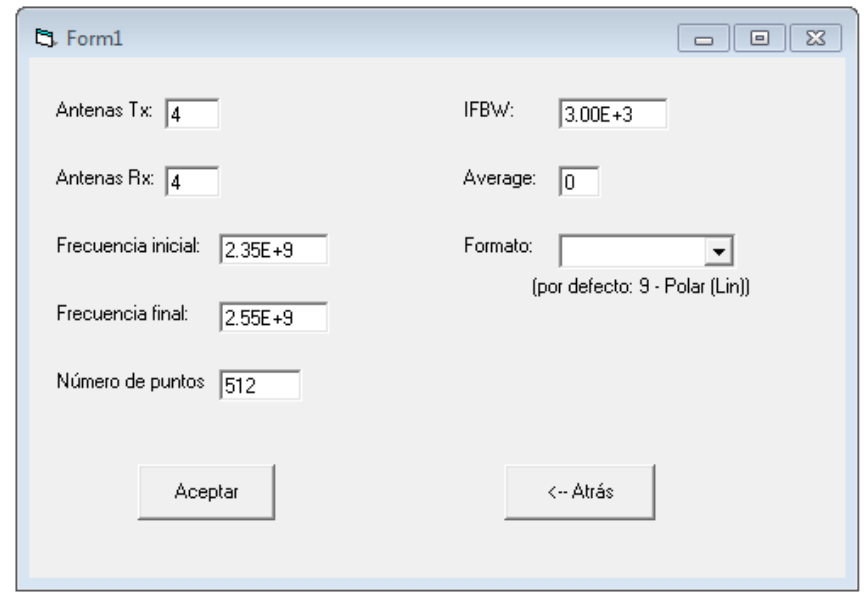

Figure C.5. Configuration window of the software application.

\section{C.2. Measurement Process}

The measurement process is divided into three steps. The first one is the configuration of the radio parameters (see previous section), the second one is the calibration process of all cables, connector and amplifiers and the third one is the measurement acquisition.

Once the configuration parameters are introduced in the software (see Section C.1.2), the application sends instructions via LAN and GPIB connections, to each devices to configure them adequately. 


\section{C.2.1. Calibration}

The antennas are connected to the MVNA and the switch using cables, amplifiers and connectors. These elements introduce attenuation and phase shifts. Therefore, a calibration procedure is required to eliminate these effects. The calibration must be done for each pair of antennas ( $M \times N$ combinations) by connecting each transmitter with each receiver. It should be remarked that since the antennas are not included in calibration, the effect of the antennas is included in measurement.

For the calibration procedure the MVNA should be configured as follows:

- Format Logmag.

- Trigger Continous.

- Adequate output power in order not to saturate the MVNA input.

- Press Calibration $\rightarrow$ Response(Thru)

This process is repeated for each pair of antennas of the MIMO system.

Once the calibration procedure is finished the initial MVNA configuration should be restored.

\section{C.2.2. Measurement Routine}

The acquisition process starts switching to the first transmitting antenna element, and then the MVNA saves the signals received by all receiving antenna elements at the same time. After that, the transmitter switches to the next transmitting antenna element and so on (see Figure C.6). This process is repeated the number of times defined by the user in the software application. It is important to note that the MVNA can read all the ports almost simultaneously (semi-switched scheme), so it makes the measurement process faster.

Once the measurement routine has finished, the user can access to measured data in the MVNA folder specified in the measurement application. 


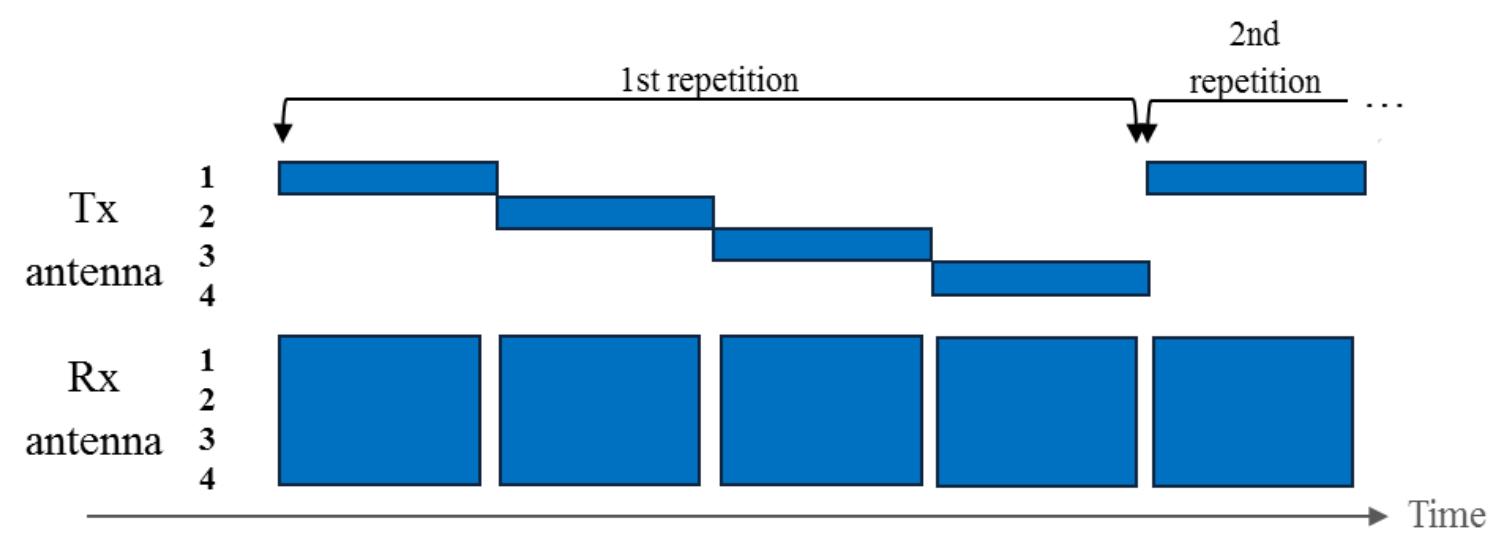

Figure C.6. Chronogram of the measurement routine. 


\section{Appendix D}

\section{GEOGRAPHIC INFORMATION SYSTEMS}

In Chapter2, Section 2.2.2 the application that controls the measurement routine of the MIMO frequency-domain channel sounder was modified in order to georeference all the measurements. Such improvement was carried out thanks to the use of a Geographical Information System (GIS) such as ArcGIS.

Thus, this appendix is devoted to Geographical Information Systems. Section D.1 defines what a GIS is. Section D.2 presents the different ways in which data is represented in a GIS. Finally, in Section D.3 the GIS used in this thesis, ArcGIS and particularly, ArcView is explained.

\section{D.1. What is a Geographic Information System?}

A Geographic Information System (GIS), is a system designed to capture, store, manipulate, analyze, manage, and present all types of geographically referenced data. In other words, a GIS is the association of cartography, statistical analysis, and database technology. GIS applications are also tools that allow users to analyze spatial information, edit data, maps, and present the results of all these operations. The main functions of a GIS are: 
Data Input - A GIS can receive the information via several sources: direct digitalization, input of the coordinates from an external file or teledection.

Data Output - Data output in a GIS is mainly via the creation of maps.

Data Management - A GIS provides tools to manage the information such as mathematic functions to carry out geometry transformations or operate with different coordinate systems. Moreover a GIS should also provide functions to import/export data to other platforms.

Analysis - Is the most important function of a GIS. The information available in a GIS is used to produce new information. To accomplish this, the GIS provides functions to determine the proximity of objects, search other objects or manage the interoperability between maps.

A GIS can be used for many and diverse applications. In agriculture to control and survey the fields; in emergencies and safety services to design and improve the service; in environmental conservation to evaluate risks and propose new methods to preserve the environment; in telecommunications to plan and manage wireless communication systems; or in defense and intelligence to improve militar planning.

A GIS processes any data that has a spatial component. This information is quite diverse and can be aerial photographs or satellite imagery, a collection of terrain contours, digital maps of the built environment, or legal records of land ownership [Zeiler, 1999]. In the following section the data representation in a GIS is detailed.

\section{D.2. Data Representation}

With a GIS, you can model geographic information or data in three basic ways: as a collection of discrete features in vector format, as a grid of cells with spectral or attribute data (raster), or as a set of triangulated points modeling a surface [Zeiler, 1999] as explained below. Choosing and adequate data representation depends on the requirements of the application or the analysis to carry out.

Descriptive information provides the user information about the geographic information (for example, the kind of river, or the kind of mountain in the map). In other words, descriptive information describes the geographic information. Such information is stored by means of attribute tables (shown in Figure D.1) which relates geographic and descriptive information using an identifier.

\section{Vector}

Vector data (see Figure D.2) represents features as points, lines, and polygons and is best applied to discrete objects with defined shapes and boundaries. Features have a precise shape and position, attributes and metadata, and useful behavior. 


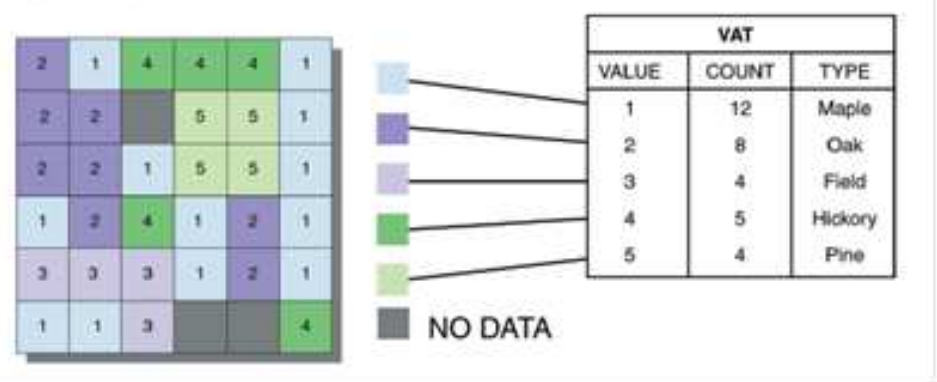

Figure D.1. Attribute table (descriptive information) related with a raster (geographic information) [ESRI, 2002].

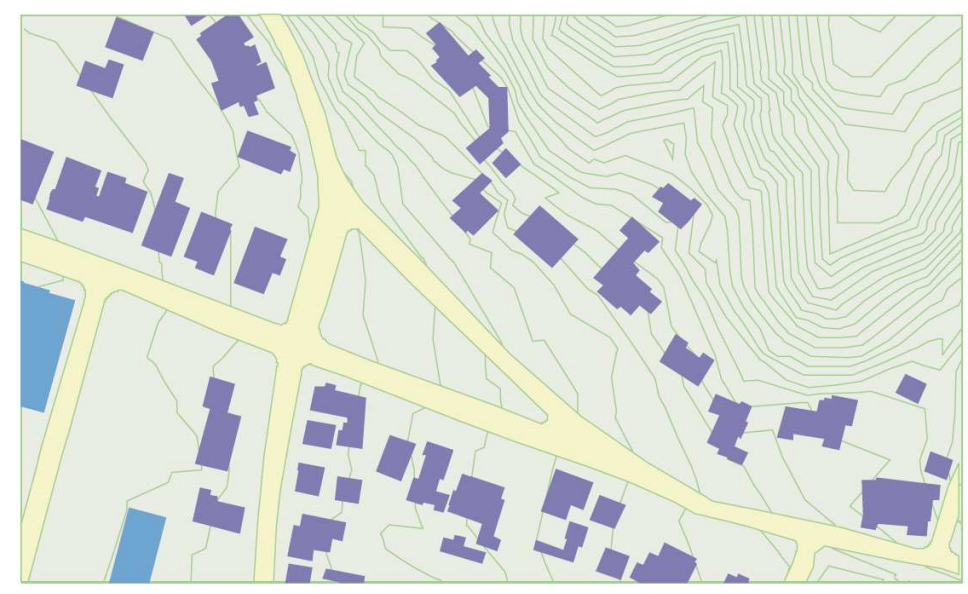

Figure D.2. Vector representation [Zeiler, 1999].

\section{Raster}

Raster data (see Figure D.3)represents imaged or continuous data. Each cell (or pixel) in a raster is a measured quantity. The most common source for a raster dataset is a satellite image or aerial photograph. A raster dataset can also be a photograph of a feature, such as a building [Zeiler, 1999].

Raster datasets excel in storing and working with continuous data, such as elevation, water table, pollution concentration, and ambient noise level.

\section{Triangulated Data}

A Triangulated Irregular Network (TIN) is a useful and efficient way to capture the surface of a piece of land (3D data) as shonw in Figure D.4.

TINs support perspective views. You can drape a photographic image on top of a TIN for a photorealistic terrain display. TINs are particularly useful for modeling watersheds, visibility, lineofsight, slope, aspect, ridges and rivers, and volumetrics.

TINs can model points, lines, and polygons. A triangulation is made of many mass points, each 


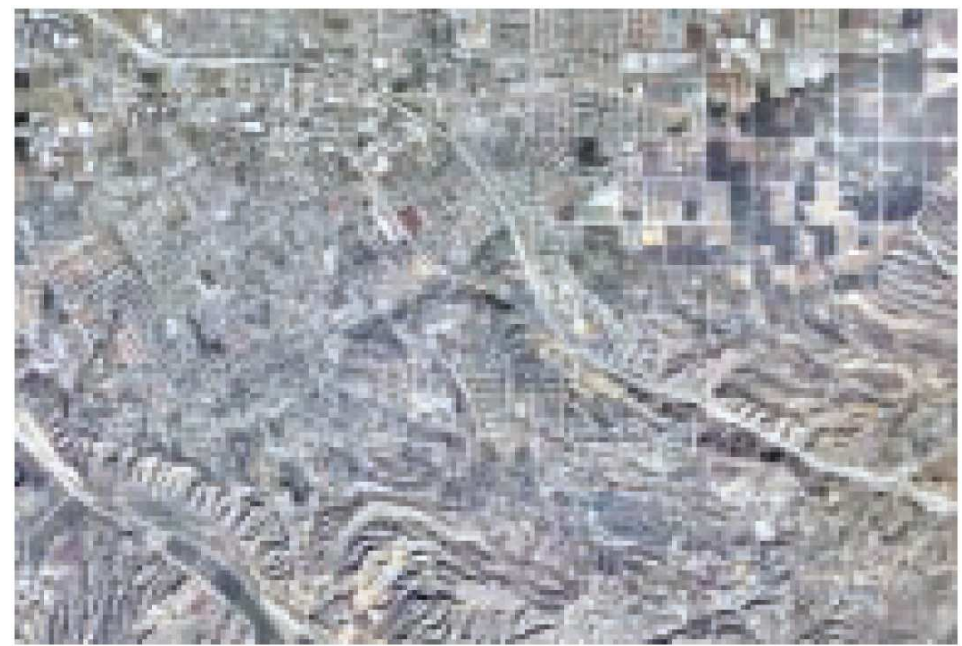

Figure D.3. Raster representation [Zeiler, 1999].

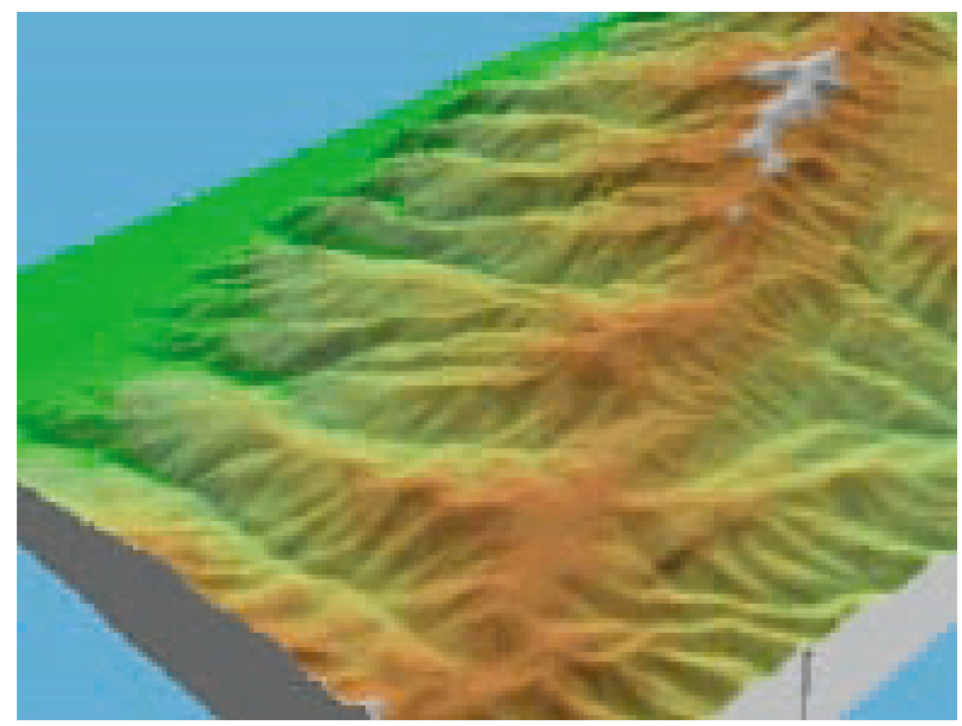

Figure D.4. TIN representation [Zeiler, 1999].

an $x, y, z$ tuple. Breaklines represent streams, ridges, and other linear discontinuities. Exclusion areas represent polygons with same elevation, such as lakes or project boundaries. Contour maps can be generated from a TIN, using linear interpolation or a smoothing algorithm.

\section{D.3. ArcGIS}

ArcGIS is a GIS from the company ESRI that lets you easily author data, maps, globes, and models on the desktop and serve them out for use on a desktop, in a browser, or in the field via mobile devices, depending on the needs of your organization. For developers, ArcGIS provides the user with tools for building user-designed applications [ESRI, 2011]. It consists of a number of frameworks for deploying GIS [ESRI, 2005]: 
- ArcGIS Desktop - An integrated suite of professional GIS applications.

- ArcGIS Engine - Embeddable developer components for building custom GIS applications.

- Server GIS - Is a GIS server and it is composed of ArcSDE, ArcIMS, and ArcGIS Server.

- Mobile GIS — Is a GIS for mobile and tablet PC applications.

ArcGIS is based on ArcObjects, a common, modular library of shared GIS software components as shown in Figure D.5. ArcObjects includes a wide variety of programmable components, ranging from individual geometry objects to map objects, which aggregate comprehensive GIS functionality for developers.

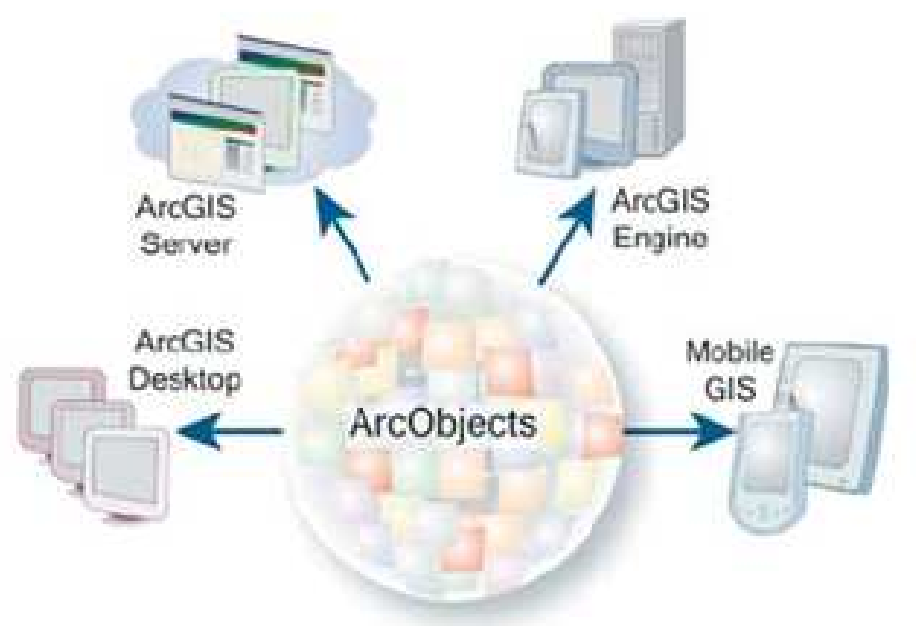

Figure D.5. ArcObjects is a common library for ArcGIS products.

In this thesis, ArcGIS Desktop has been used. Therefore, in the next section this specific product from ESRI is explained.

\section{D.3.1. ArgGIS Desktop, ArcView and ArcMap}

ArcGIS Desktop is a product from ArcGIS that perform advanced spatial analysis, model operational processes, and visualize results on professional-quality maps. It is an integrated suite of desktop applications for Windows such as ArcMap, ArcCatalog, ArcToolbox and ArcGlobe. ArcGIS Desktop is available at three functional levels:

- ArcView - This package focuses on comprehensive data use, mapping and analysis.

- ArcEditor - This package adds to ArcView advance geographic editing and data creation features. 
- ArcInfo - This package is the most powerful from ArcGIS Desktop. It is a complete, professional GIS desktop containing comprehensive GIS functionality, including rich geoprocessing tools.

In this thesis, ArcMap under the ArcView level has been used. ArcMap is the central application in ArcGIS Desktop [ESRI, 2005] for tasks based on maps management. It includes cartography, map analysis and editing.

ArcMap offers two types of map views: a geographic data view and a page layout view. In the geographic data view, users work with geographic layers to symbolize, analyze, and compile GIS datasets. A table of contents interface helps organize and control the drawing properties of the GIS data layers in the data frame.

In the layout view, users work with map pages that contain geographic data views as well as other map elements, such as scalebars, legends, North arrows, and reference maps. This view is used to creates maps for printing and publishing.

As mentioned before, the user can build applications to run in ArcGIS via the library ArcObjects. User-defined toolbars can be added to ArcMap and in order to improve the user's work. For this purpose, an editor of Visual Basic for Applications is embedded. This characteristic or ArcGIS has been used in this thesis to implement a measurement application in ArcMap. 


\section{LIST OF FIGURES}

2.1. Direct RF Pulse Scheme $[$ Rappaport, 1996]. . . . . . . . . . . . . . . . . . . . 11

2.2. Spread Spectrum Sliding Correlator Channel Sounding [Rappaport, 1996]. . . . . . . . 11

2.3. Frequency Domain Channel Sounding $[$ Rappaport, 1996]. . . . . . . . . . . . . . . 12

2.4. Fully-switched configuration $\mid$ Laureson, 2006]. . . . . . . . . . . . . . . . 14

2.5. Fully-parallel configuration $[$ Laureson, 2006] . . . . . . . . . . . . . . . . 14

2.6. Frequency-domain channel sounder. . . . . . . . . . . . . . . 15

2.7. Software application Lucas Ávila, 2008] (a) main interface, (b) configuration parame-

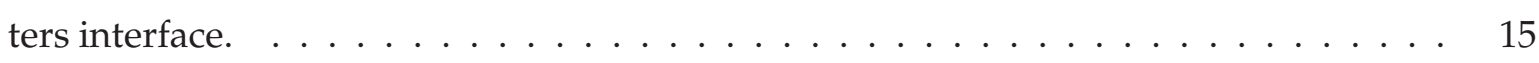

2.8. $\mathrm{XY}$ positioning board with the transmitting antenna. . . . . . . . . 16

2.9. XY linear positioners Robotics, 2011] . . . . . . . . . . . . . . . . 17

2.10. Step motors and controller $[$ Robotics, 2011] . . . . . . . . . . . . . . . . 17

2.11. Modified interface to introduce the configuration parameters of the $X Y$ positioning system Alcaraz Vidal, 2010]. . . . . . . . . . . . . . . . . . . . . 18

2.12. Scheme of the room where measurements were performed. . . . . . . . . . . 18 
2.13. Photo of the laboratory where measurements were undertaken (a) View from the door,

(b) View from the LOS 1 position.

2.14. Mean RMS delay spread when the transmitter is located at (a) LOS 1 and (b) LOS 2. .

2.15. Mean RMS delay spread when the transmitter is located at (a) NLOS1 and (b) NLOS2.

2.16. Correlation coefficient at the receiver for LOS 1

2.17. Correlation coefficient at the receiver for $\operatorname{LOS} 2 \ldots \ldots \ldots \ldots \ldots \ldots$

2.18. Correlation coefficient at the receiver for NLOS 1.

2.19. Correlation coefficient for at the receiver NLOS 2.

2.22. Folders created by the application in the MVNA hard disk.

2.23. Block Diagram of the time-domain MIMO channel sounder.

2.24. LS codes used in the channel sounder.

2.25. Autocorrelation and crosscorrelation of the LS codes.

2.26. (b) LS code after rised-cosine pulse shaping in time domain (b) Normalized Spectrum of the code.

2.28. Block diagram of the measurement procedure.

2.31. Agilent 90804 A oscilloscope. . . . . . . . . . . . . . . . . . . .

2.32. (a) ZVE-8G+ amplifier, (b) VBFZ passband filter, both from MiniCircuits [MiniCircuits, ]. 34

2.33. Electrometrics EM-6116 antenna.

2.34. (a) D-Link Wi-Fi card, (b) Agilent USB/GPIB cable. 
2.36. Misalignment between trigger and received signals. . . . . . . . . . . . 37

2.37. Measurement environment. . . . . . . . . . . . . . . . . . . . . 41

2.38. Normalized power-delay profile of the MIMO transfer matrix. . . . . . . . . . . . 43

3.1. Emissions regulation in Europe (EC) and the United States (FCC).. . . . . . . . . . 47

3.2. Floor plan of the measurement scenario and the position of the transmitting and receiving antennas. . . . . . . . . . . . . . . . . . . 49

3.3. Block diagram of the MIMO channel sounder. . . . . . . . . . . . . . 49

3.4. Different polarizations for the arrays. . . . . . . . . . . . . . . 51

3.5. Mean path loss for TX-Loc2 and $\mathrm{M}_{21}+\mathrm{M}_{22}$, and $\mathrm{M}_{23}$ and the adjusted models. . . . . 53

3.6. Averaged path loss for TX-Loc 2 and $\mathrm{M}_{21}+\mathrm{M}_{22}$, as a function of the central frequency of the $500 \mathrm{MHz}$ frequency interval. . . . . . . . . . . . . . . . 55

3.7. $X P D$ as function of distance for each TX-RX pair: (a) $X P D_{V}$ and (b) $X P D_{H} \ldots \ldots$

3.8. Averaged path loss for TX-Loc1 and $\mathrm{M}_{1}$ and the adjusted model. . . . . . . . . . 59

3.9. $X P D$ as function of distance for each TX-RX pair: (a) $X P D_{V}$ and (b) $X P D_{H} \ldots \ldots 1$

3.10. RMS delay spread for the group of measurements $M_{21}$ and $M_{22}$ (corridor in LoS). . . 63

3.11. RMS delay spread as a function of the central frequency for zone $\mathrm{M}_{21}$ (corridor in LoS). 64

3.12. RMS delay spread for the group of measurements $\mathrm{M}_{1} \ldots \ldots \ldots \ldots$

3.13. RMS delay spread as a function of the central frequency for zone $\mathrm{M}_{1} \ldots \ldots$

3.14. CCDFs of the capacity for the group of measurements $M_{21}+M_{22}$ for different

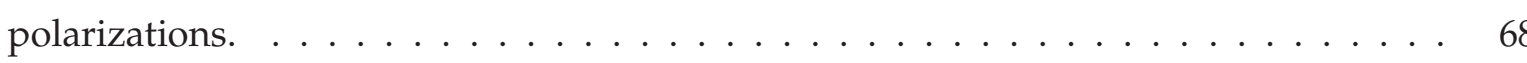

3.15. CCDFs of the capacity for the group of measurements $M_{1}$ for different polarizations. . $\quad 69$

3.16. (a) Received power and (b) the mean measured capacity as a function of distance for a variable SNR in the main corridor $\left(\mathrm{M}_{21}+\mathrm{M}_{22}\right)$ in LoS. . . . . . . . . . . . . . 71

3.17. (a) Received power and (b) the mean measured capacity as a function of distance for a

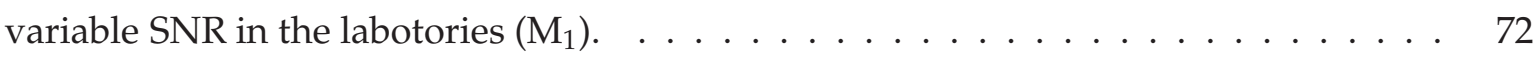




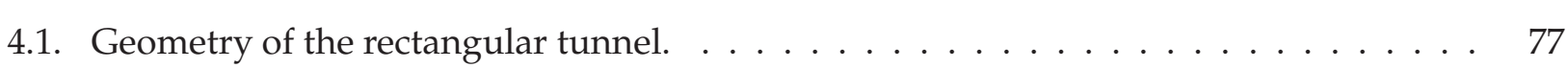

4.2. Situation in case of several receivers. . . . . . . . . . . . . . 83

4.3. Photo of the tunnel where measurements were carried out. . . . . . . . . . . . . 83

4.4. Scheme of the channel sounder set up. . . . . . . . . . . . . . 84

4.5. Configuration of the wideband measurements. . . . . . . . . . . 85

4.6. Mean path loss versus distance. . . . . . . . . . . . . . . . . . . . . . 87

4.7. Successive positions of $\mathrm{Rx}$ for calculating the standard deviation of the path loss. $L=8$ $\mathrm{m}$ for $d \leq 200 \mathrm{~m}$ and $L=12 \mathrm{~m}$ for $206 \mathrm{~m} \leq d \leq 486 \mathrm{~m}$. . . . . . . . . . . 87

4.8. Transverse correlation for $f=2.8 \mathrm{GHz}$ and $f=5 \mathrm{GHz}$ for different antenna spacings,

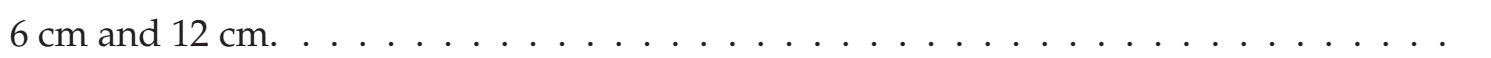

4.9. Mean transverse correlation for different antenna spacings, $6 \mathrm{~cm}$ and $12 \mathrm{~cm}$. . . . .

4.10. Axial correlation coefficient versus distance $d$ between 2 points, $4 \mathrm{~m}$ apart (if $d<204$ $\mathrm{m})$ or $6 \mathrm{~m}$ apart $($ if $d>204 \mathrm{~m}) \ldots \ldots \ldots \ldots \ldots \ldots$

4.11. PDPs deduced from measurements and normalized to $0 \mathrm{~dB}$ corresponding to their

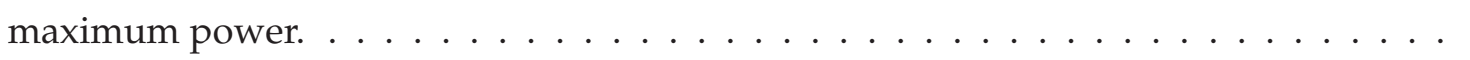

4.12. PDPs obtained from a theoretical propagation model, assuming a tunnel of rectangular cross section $(8 \times 5.6 \mathrm{~m})$ and the same frequency bandwidth as in the measurements. .

4.13. CCDFs of the local $\mathrm{rms}$ delay spread deduced from experimental data. . . . . . . . .

4.14. Scheme of the double directional channel. . . . . . . . . . . . . . . 94

4.15. Normalized theoretical $A P S(d, \varphi)$, in $\mathrm{dB}$, related to the angle of arrival (AoA). . . . 96

4.16. Normalized theoretical APS $(d, \varphi)$, in $\mathrm{dB}$, related to the angle of arrival (AoA) and obtained by the high resolution algorithm from the theoretical channel transfer functions. 97

4.17. Normalized $\operatorname{APS}(d, \varphi)$ of the angle of arrival (AoA) expressed in $\mathrm{dB}$ deduced from measurements. . . . . . . . . . . . . . . . . . . .

4.18. RMS angular spread versus the distance between Tx and Rx, deduced from measurements (both AoA and AoD from measurements) and from a ray tracing approach (theoretical). 
4.19. Coherence bandwidth $(\rho=0.7)$ versus distance calculated for different values of the correlation coefficient.

4.20. Frequency diversity degree versus distance for a total band used of $B_{p}=500 \mathrm{MHz}$. . . 101

4.21. Correlation coefficient versus frequency separation and antenna element spacing for distance $T x-R x=50 \mathrm{~m}$.

4.22. Correlation coefficient versus frequency separation and antenna element spacing for distance $\mathrm{Tx}-\mathrm{Rx}=446 \mathrm{~m}$

4.23. Mean MIMO capacity assuming a fixed SNR at the receiver of $15 \mathrm{~dB}$.

4.24. Average relative received power for various polarizations. The reference value is $0 \mathrm{~dB}$ at $50 \mathrm{~m}$ and for VV polarization.

4.25. Mean MIMO capacity assuming a fixed Tx Power. . . . . . . . . . . . . . . . . . 106

4.26. Waveforms of the (a) transmitted pulse, and (b) to (d) of the received signals at different distances.

4.27. Received signal with TR: (a) $d=d_{0}=50 \mathrm{~m}$, (b) $d=54 \mathrm{~m}$ but $d_{0}=50 \mathrm{~m}$, (c) $d=58 \mathrm{~m}$ but $d_{0}=50$.

4.28. Waveform of the received signals at (a) $d=50 \mathrm{~m}$ with SISO-TR, (b) $d=50 \mathrm{~m}$ with MISO-TR, (c) $d=200 \mathrm{~m}$ with SISO-TR, (d) $d=200 \mathrm{~m}$ with MISO-TR, (e) $d=498 \mathrm{~m}$ with SISO-TR, (b) $d=498 \mathrm{~m}$ with MISO-TR.

4.29. Average rms delay spread: SISO without TR, SISO TR and MISO TR.

4.30. CDF of $R_{S I S O-T R}$ and $R_{M I S O-T R}$ for short range $(d<100 \mathrm{~m})$ and for long range (400 $\mathrm{m}<d<500 \mathrm{~m}$ ) communication.

4.31. Peak to peak gain for SISO TR and MISO TR configurations.

A.1. Multiantenna configurations: (a) SISO, (b) SIMO, (c) MISO and (d) MIMO. . . . . . . 126

A.2. Benefits that MIMO can offer. . . . . . . . . . . . . . . . . . . 127

A.3. Directivity or diversity at the receiving and transmitting arrays determine if the channel supports beamforming, diversity, or spatial multiplexing. 
C.1. Block diagram of the frequency domain channel sounder. . . . . . . . . . . . . . . . 140

C.2. Agilent Multiport Vector Network Analyzer. . . . . . . . . . . . . . . . . . . . 141

C.3. Agilent Switch and controller. . . . . . . . . . . . . . . . . . . . 142

C.4. Main interface of the software application. . . . . . . . . . . . . 143

C.5. Configuration window of the software application. . . . . . . . . . 143

C.6. Chronogram of the measurement routine. . . . . . . . . . . . . . . . . 145

D.1. Attribute table (descriptive information) related with a raster (geographic information) [ESRI, 2002]. . . . . . . . . . . . . . . . . . . . . . . . 149

D.2. Vector representation $[$ Zeiler, 1999]. . . . . . . . . . . . . . . . . . . . . 149

D.3. Raster representation $[$ Zeiler, 1999] . . . . . . . . . . . . . . . . . . . . . . 150

D.4. TIN representation $[$ Zeiler, 1999]. . . . . . . . . . . . . . . . . . . . . . 150

D.5. ArcObjects is a common library for ArcGIS products. . . . . . . . . . . . . . . . . . 151 


\section{LisT OF TABLES}

2.1. Configuration parameters of the frequency-domain channel sounder. . . . . . . . . 19

2.2. Mean and standard deviation of the RMS delay spread for each position. . . . . . . . 21

2.3. Correlation distance for each position of the transmitting array. . . . . . . . . . . 21

2.4. Different filters used in the channel sounder. . . . . . . . . . . . . . 35

2.5. Minimum voltage and power that can be measured by the oscilloscope. . . . . . . 38

2.6. Minimum acceptable power in the oscilloscope depending on the vertical scale. . . . 38

2.7. Gain in the transmitting side. . . . . . . . . . . . . . . . . . . . . . . . .

2.8. Gain in the receiving side. . . . . . . . . . . . . . . . . . . . . . . . .

2.9. Measurement Parameters. . . . . . . . . . . . . . . . . . . . . . . 41

2.10. Configuration parameters of the time-domain channel sounder. . . . . . . . . . 42

2.11. Configuration parameters of the frequency-domain channel sounder. . . . . . . . . 42

2.12. RMS delay spread and correlation values for both channel sounders. . . . . . . . . 43 
3.1. Whole bandwidth model parameters for the group of measurements $\mathrm{M}_{21}+\mathrm{M}_{22}$ and

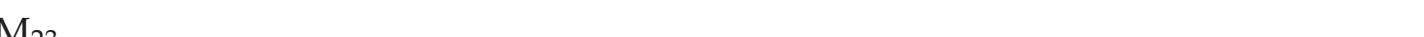

3.2. Decay factor as a function of the central frequency in $\mathrm{M}_{21}+\mathrm{M}_{22}$ zones. . . . . . . . . 5

3.3. $L_{0}$ in decibels as a function of the central frequency in $\mathrm{M}_{21}+\mathrm{M}_{22}$ zones. . . . . . . 55

3.4. Mean and standard deviation of the XPD in zones $\mathrm{M}_{21}+\mathrm{M}_{22}$ and $\mathrm{M}_{23} \ldots \ldots \ldots 7$

3.5. Mean and standard deviation of the XPD as a function of the central frequency $(\mathrm{GHz})$

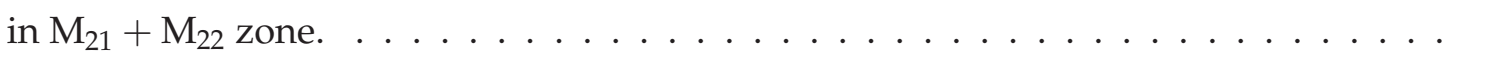

3.6. Mean and standard deviation of the $X P D$ as a function of the central frequency $(\mathrm{GHz})$

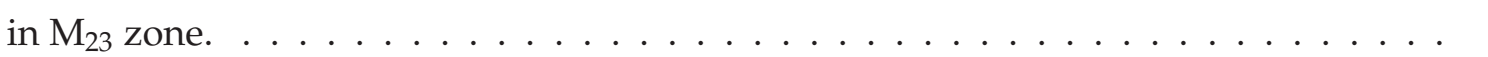

3.7. Whole bandwidth parameters of the proposed model for $\mathrm{M}_{1}$ zone. . . . . . . . . . 60

3.8. Whole bandwidth parameters for a one-slope model for $\mathrm{M}_{1}$ zone. . . . . . . . . . . . 60

3.9. Mean and standard deviation of the $X P D$ in zone $\mathrm{M}_{1} \ldots \ldots \ldots$. . . . . . . .

3.10. Mean and standard deviation of the $X P D$ as a function of the central frequency $(\mathrm{GHz})$

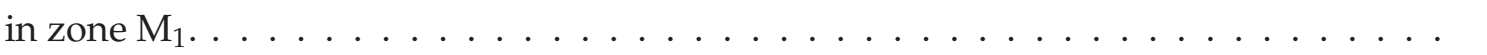

3.11. Correlations and capacity for SNR fixed at $10 \mathrm{~dB}$ when probability $>0.9$ in corridor

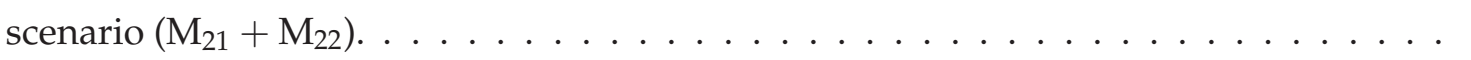

3.12. Correlations and capacity for SNR fixed at $10 \mathrm{~dB}$ when probability $>0.9$ in laboratories

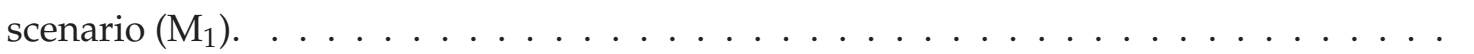

4.1. Equipment characteristics and measurement parameters. . . . . . . . . . . . . . .

4.2. Average value of the capacity far from the transmitter using either co-polarized vertical antennas or dual-polarized antennas of different lengths. . . . . . . . . . . . . . 107 


\section{BIBLIOGRAPHY}

[Alcaraz Vidal, 2010] Alcaraz Vidal, V. (2010). Implementación de un sistema de posicionamiento de antenas para la automatización de medidas del canal radio. Technical report, ETSIT - Final Degree Project.

[Alonso Sánchez, 2010] Alonso Sánchez, E. (2010). Implementación de un sistema de posicionamiento de antenas para la automatización de medidas del canal radio. Technical report, ETSIT - Final Degree Project.

[Barton et al., 2007] Barton, R. J., Chen, J., Huang, K., Wu, D., and Wu, H.-C. (2007). Performance of cooperative time-reversal communication in a mobile wireless environment. Int. J. Distrib. Sen. Netw., 3:59-68.

[Baum et al., 2000] Baum, D., Gore, D., Nabar, R., Panchanathan, S., Hari, K., Erceg, V., and Paulraj, A. (2000). Measurement and characterization of broadband mimo fixed wireless channels at 2.5 ghz. In Personal Wireless Communications, 2000 IEEE International Conference on, pages 203 -206.

[Bello, 1963] Bello, P. (1963). Characterization of randomly time-variant linear channels. Communications Systems, IEEE Transactions on, 11(4):360 -393.

[Benedetto et al., 2006] Benedetto, M.-G. D., Kaiser, T., Molisch, A. F., Oppermann, I., Politano, C., and Porcino, D. (2006). UWB Communication Systems: A Comprehensive Overview. EURASIP Book Series on Signal Processing and Communications, Volume 5. 
[Bhagavatula et al., 2010] Bhagavatula, R., Oestges, C., and Heath, R. (2010). A new doubledirectional channel model including antenna patterns, array orientation, and depolarization. Vehicular Technology, IEEE Transactions on, 59(5):2219 -2231.

[Boutin et al., 2008] Boutin, M., Benzakour, A., Despins, C., and Affes, S. (2008). Radio wave characterization and modeling in underground mine tunnels. Antennas and Propagation, IEEE Transactions on, 56(2):540 -549.

[Choi et al., 2009] Choi, J., Kang, N.-G., Sung, Y.-S., Kang, J.-S., and Kim, S.-C. (2009). Frequencydependent $\mathrm{uwb}$ channel characteristics in office environments. Vehicular Technology, IEEE Transactions on, 58(7):3102 -3111.

[Choi et al., 2006a] Choi, J., Kang, N.-G., Sung, Y.-S., and Kim, S.-C. (2006a). Empirical ultra wide band path loss model in office environments. In Vehicular Technology Conference, 2006. VTC 2006Spring. IEEE 63rd, volume 4, pages $1956-1960$.

[Choi et al., 2006b] Choi, J. H., Chung, H. K., Lee, H., Cha, J., and Lee, H. (2006b). Code-division multiplexing based mimo channel sounder with loosely synchronous codes and kasami codes. In Vehicular Technology Conference, 2006. VTC-2006 Fall. 2006 IEEE 64th, pages 1 -5.

[Chong et al., 2005] Chong, C.-C., Kim, Y.-E., Yong, S. K., and Lee, S.-S. (2005). Statistical characterization of the uwb propagation channel in indoor residential environment: Research articles. Wirel. Commun. Mob. Comput., 5:503-512.

[Chung et al., 2005] Chung, H. K., Vloeberghs, N., Kwon, H. K., Lee, S. J., and Lee, K. C. (2005). Mimo channel sounder implementation and effects of sounder impairment on statistics of multipath delay spread. In Vehicular Technology Conference, 2005. VTC-2005-Fall. 2005 IEEE 62nd, volume 1, pages $349-353$.

[Ciccognani et al., 2005] Ciccognani, W., Durantini, A., and Cassioli, D. (2005). Time domain propagation measurements of the uwb indoor channel using pn-sequence in the fcc-compliant band 3.6-6 ghz. Antennas and Propagation, IEEE Transactions on, 53(4):1542 - 1549.

[Dudley et al., 2007] Dudley, D., Lienard, M., Mahmoud, S., and Degauque, P. (2007). Wireless propagation in tunnels. Antennas and Propagation Magazine, IEEE, 49(2):11 -26.

[Dudley, 1994] Dudley, D. G. (1994). Mathematical Foundations for Electromagnetic Theory. IEEE Press.

[ECO, 2009] ECO, E. C. O. (2009). Commission decision of the european communities on allowing the use of the radio spectrum for equipment using ultra-wideband technology in a harmonised manner in the community-2009/343/ec.

[Eggers et al., 1993] Eggers, P., Toftgard, J., and Oprea, A. (1993). Antenna systems for base station diversity in urban small and micro cells. Selected Areas in Communications, IEEE Journal on, 11(7):1046 -1057. 
[Eggers, 1999] Eggers, P. C. F. (1999). Angular propagation descriptions relevant for base station adaptive antenna operations. Wirel. Pers. Commun., 11:3-29.

[Eiceg et al., 2006] Eiceg, V., Sampath, H., and Catreux-Erceg, S. (2006). Dual-polarization versus single-polarization mimo channel measurement results and modeling. Wireless Communications, IEEE Transactions on, 5(1):28 - 33.

[Emslie et al., 1975] Emslie, A., Lagace, R., and Strong, P. (1975). Theory of the propagation of uhf radio waves in coal mine tunnels. Antennas and Propagation, IEEE Transactions on, 23(2):192 - 205.

[Erceg et al., 2004] Erceg, V., Soma, P., Baum, D., and Catreux, S. (2004). Multiple-input multipleoutput fixed wireless radio channel measurements and modeling using dual-polarized antennas at 2.5 ghz. Wireless Communications, IEEE Transactions on, 3(6):2288 - 2298.

[ESRI, 2002] ESRI (2002). Using ArcGIS Spatial Analyst:.

[ESRI, 2005] ESRI (2005). What is ArcGIS 9.1?

[ESRI, 2011] ESRI (2011). Arcgis. www.esri.com.

[Fan, 2004] Fan, P. (2004). Spreading sequence design and theoretical limits for quasisynchronous cdma systems. EURASIP J. Wirel. Commun. Netw., 2004:19-31.

[FCC, 2002] FCC, F. C. C. (2002). First report and order 02-48.

[Fernandez et al., 2005] Fernandez, O., Domingo, M., and Torres, R. (2005). Empirical analysis of the correlation of mimo channels in indoor scenarios at $2 \mathrm{ghz}$. Communications, IEE Proceedings-, 152(1):82 - 88 .

[Fessler and Hero, 1994] Fessler, J. and Hero, A. (1994). Space-alternating generalized expectationmaximization algorithm. Signal Processing, IEEE Transactions on, 42(10):2664 -2677.

[Fink et al., 2000] Fink, M., Cassereau, D., Derode, A., Prada, C., Roux, P., Tanter, M., Thomas, J.-L., and Wu, F. (2000). Time-reversed acoustics. Reports on Progress in Physics, 63(12):1933.

[Foschini and Gans, 1998] Foschini, G. J. and Gans, M. J. (1998). On limits of wireless communications in a fading environment when using multiple antennas. Wireless Personal Commun, 6:311-335.

[Garrido Cervantes, 2010] Garrido Cervantes, A. (2010). Implementación de un sistema de posicionamiento de antenas para la automatización de medidas del canal radio. Technical report, ETSIT - Final Degree Project.

[Gesbert et al., 2003] Gesbert, D., Shafi, M., shan Shiu, D., Smith, P., and Naguib, A. (2003). From theory to practice: an overview of mimo space-time coded wireless systems. Selected Areas in Communications, IEEE Journal on, 21(3):281 - 302. 
[Ghassemzadeh et al., 2004] Ghassemzadeh, S., Jana, R., Rice, C., Turin, W., and Tarokh, V. (2004). Measurement and modeling of an ultra-wide bandwidth indoor channel. Communications, IEEE Transactions on, 52(10):1786 - 1796.

[GmbH, 2011] GmbH, M. (2011). Rusk channel sounder. www.channelsounder.de.

[Golay, 1961] Golay, M. (1961). Complementary series. Information Theory, IRE Transactions on, 7(2):82 $-87$.

[Haardt, 1996] Haardt, M. (1996). Efficient One-, Two-, and Multidimensional High-Resolution Array Signal Processing. PhD thesis, Technische Universität München.

[Henty, 2007] Henty, B. E. (2007). Advanced System Design of in-building Wireless Communication Networks using Ventilation Ducts. PhD thesis, Electrical and Electronic Eng. Dept., Carnegie Mellon Univ., Pittsburgh, USA.

[Ibernón-Fernández et al., 2008] Ibernón-Fernández, R., Molina-Garcia-Pardo, J.-M., Rodriguez, J.V., and Juan-Llacer, L. (2008). Sistema de medidas mimo de banda ancha basado en un analizador de redes multipuerto. Revista Española de electrónica, 642:80-85.

[Keignart et al., 2006] Keignart, J., Abou-Rjeily, C., Delaveaud, C., and Daniele, N. (2006). Uwb simo channel measurements and simulations. Microwave Theory and Techniques, IEEE Transactions on, 54(4):1812 - 1819.

[Kemp and Bryant, 2004] Kemp, A. H. and Bryant, E. B. (2004). Channel sounding of industrial sites in the $2.4 \mathrm{ghz}$ ism band. Wirel. Pers. Commun., 31:235-248.

[Khan and Hall, 2010] Khan, I. and Hall, P. (2010). Experimental evaluation of mimo capacity and correlation for narrowband body-centric wireless channels. Antennas and Propagation, IEEE Transactions on, 58(1):195 -202.

[Kim et al., 2008] Kim, M., Kim, S., Lee, H., Lee, H., and You, H.-R. (2008). Performance comparison of some codes in code division multiplexing based mimo channel sounder architecture. In Advanced Communication Technology, 2008. ICACT 2008. 10th International Conference on, volume 2, pages $1343-1346$.

[Kivinen et al., 1999] Kivinen, J., Korhonen, T., Aikio, P., Gruber, R., Vainikainen, P., and Haggman, S.-G. (1999). Wideband radio channel measurement system at 2 ghz. Instrumentation and Measurement, IEEE Transactions on, 48(1):39 -44.

[Kivinen et al., 2001] Kivinen, J., Zhao, X., and Vainikainen, P. (2001). Empirical characterization of wideband indoor radio channel at $5.3 \mathrm{ghz}$. Antennas and Propagation, IEEE Transactions on, 49(8):1192 -1203. 
[Kyritsi and Cox, 2002] Kyritsi, P. and Cox, D. (2002). Expression of mimo capacity in terms of waveguide modes. Electronics Letters, 38(18):1057 - 1058.

[Kyritsi et al., 2002] Kyritsi, P., Cox, D., Valenzuela, R., and Wolniansky, P. (2002). Effect of antenna polarization on the capacity of a multiple element system in an indoor environment. Selected Areas in Communications, IEEE Journal on, 20(6):1227 - 1239.

[Kyritsi et al., 2004] Kyritsi, P., Papanicolaou, G., Eggers, P., and Oprea, A. (2004). Miso time reversal and delay-spread compression for fwa channels at $5 \mathrm{ghz}$. Antennas and Wireless Propagation Letters, IEEE, 3(1):96 -99.

[Laakmann and Steier, 1976] Laakmann, K. D. and Steier, W. H. (1976). Waveguides: characteristic modes of hollow rectangular dielectric waveguides. Applied Optics, 14:1334-1340.

[Lagarias et al., 1998] Lagarias, J. C., Reeds, J. A., Wright, M. H., and Wright, P. E. (1998). Convergence properties of the nelder-mead simplex method in low dimensions. SIAM Journal of Optimization, 9:112-147.

[Laureson, 2006] Laureson, D.; Grant, P. (2006). A review of radio channel sounding techniques. In 14th European Signal Processing Conference, EUSIPCO 2006.

[Lee and Williams, 2000] Lee, K. and Williams, D. (2000). A space-frequency transmitter diversity technique for ofdm systems. In Global Telecommunications Conference, 2000. GLOBECOM '00. IEEE, volume 3, pages $1473-1477$ vol.3.

[Lerosey et al., 2004] Lerosey, G., De Rosny, J., Tourin, A., Derode, A., Montaldo, G., and Fink, M. (2004). Time reversal of electromagnetic waves. Physical Review Letters, 92(19):193904.

[Lerosey et al., 2005] Lerosey, G., de Rosny, J., Tourin, A., Derode, A., Montaldo, G., and Fink, M. (2005). Time reversal of electromagnetic waves and telecommunication. Radio Science, 40:RS6S12.1-RS6S12.10.

[Lienard et al., 2003] Lienard, M., Degauque, P., Baudet, J., and Degardin, D. (2003). Investigation on mimo channels in subway tunnels. Selected Areas in Communications, IEEE Journal on, 21(3):332 339.

[Lienard et al., 2006] Lienard, M., Degauque, P., and Molina-Garcia-Pardo, J. M. (2006). Wave propagation in tunnels in a mimo context-a theoretical and experimental study. Comptes Rendus Physique, 7:726- 734 .

[Lienard et al., 1997] Lienard, M., Lefeuvre, P., and Degauque, P. (1997). Remarks on the computation of the propagation of high frequency waves in a tunnel. Annales des Télécommunications, 52:529533. 
[Liénard and Degauque, 1999] Liénard, M. and Degauque, P. (1999). Wideband analysis of propagation along radiating cables in tunnels. Radio Science, 34:113-122.

[Liu et al., 2005] Liu, H., Qiu, R., and Tian, Z. (2005). Error performance of pulse-based ultra-wideband mimo systems over indoor wireless channels. Wireless Communications, IEEE Transactions on, 4(6):2939 - 2944.

[Liu et al., 2008] Liu, X.-F., Wang, B.-Z., Xiao, S.-Q., , and Deng, J. H. (2008). Performance of impulse radio uwb communications based on time reversal technique. Progress In Electromagnetics Research, 79:401-413.

[Lucas Ávila, 2008] Lucas Ávila, R. A. (2008). Software para la automatización de medidas de un sistema mimo de banda ancha. Technical report, ETSIT - Final Degree Project.

[Maharaj et al., 2005] Maharaj, B., Linde, L., Wallace, J., and Jensen, M. (2005). A cost-effective wideband mimo channel sounder and initial co-located $2.4 \mathrm{ghz}$ and $5.2 \mathrm{ghz}$ measurements. In Acoustics, Speech, and Signal Processing, 2005. Proceedings. (ICASSP'05). IEEE International Conference on, volume 3, pages iii/981 - iii/984 Vol. 3.

[Mahmoud and Wait, 1974] Mahmoud, S. and Wait, J. (1974). Geometrical optical approach for electromagnetic wave propagation in rectangular mine tunnels. Radio Scienci, 9:1147-1158.

[Mahmoud, 1998] Mahmoud, S. D. (1998). Electromagnetic waveguides theory and applications. Peter Peregrinus Ltd.

[Mahmoud, 2010] Mahmoud, S. F. (2010). Mobile and Wireless Communications. Chapter 1 Wireless transmission in tunnels. InTech.

[Malik, 2008a] Malik, W. (2008a). Polarimetric characterization of ultrawideband propagation channels. Antennas and Propagation, IEEE Transactions on, 56(2):532 -539.

[Malik, 2008b] Malik, W. (2008b). Spatial correlation in ultrawideband channels. Wireless Communications, IEEE Transactions on, 7(2):604 -610.

[Malik and Edwards, 2007] Malik, W. and Edwards, D. (2007). Measured mimo capacity and diversity gain with spatial and polar arrays in ultrawideband channels. Communications, IEEE Transactions on, 55(12):2361 -2370.

[Mariage et al., 1994] Mariage, P., Lienard, M., and Degauque, P. (1994). Theoretical and experimental approach of the propagation of high frequency waves in road tunnels. Antennas and Propagation, IEEE Transactions on, 42(1):75 -81.

[MiniCircuits, ] MiniCircuits. http://www.minicircuits.com/. 
[Molina-Garcia-Pardo et al., 2008a] Molina-Garcia-Pardo, J., Lienard, M., Degauque, P., Dudley, D., and Juan-Llacer, L. (2008a). Interpretation of mimo channel characteristics in rectangular tunnels from modal theory. Vehicular Technology, IEEE Transactions on, 57(3):1974 -1979.

[Molina-Garcia-Pardo et al., 2008b] Molina-Garcia-Pardo, J., Lienard, M., Nasr, A., and Degauque, P. (2008b). On the possibility of interpreting field variations and polarization in arched tunnels using a model for propagation in rectangular or circular tunnels. Antennas and Propagation, IEEE Transactions on, 56(4):1206-1211.

[Molina-Garcia-Pardo et al., 2011] Molina-Garcia-Pardo, J. M., Lienard, M., and Degauque, P. (2011). Digital Communication. Chapter 4: Wireless Communication in Tunnels. InTech.

[Molina-Garcia-Pardo et al., 2003] Molina-Garcia-Pardo, J.-M., Rodriguez, J.-V., and Juan-Llacer, L. (2003). Angular spread at $2.1 \mathrm{ghz}$ while entering tunnels. Microwave and Optical Technology Letters, 37(3):196-198.

[Molina-Garcia-Pardo et al., 2004] Molina-Garcia-Pardo, J.-M., Rodriguez, J.-V., and Juan-Llacer, L. (2004). Wide-band measurements and characterization at $2.1 \mathrm{ghz}$ while entering in a small tunnel. Vehicular Technology, IEEE Transactions on, 53(6):1794 - 1799.

[Molina-Garcia-Pardo et al., 2008c] Molina-Garcia-Pardo, J.-M., Rodriguez, J.-V., and Juan-Llacer, L. (2008c). Polarized indoor mimo channel measurements at $2.45 \mathrm{ghz}$. Antennas and Propagation, IEEE Transactions on, 56(12):3818 -3828.

[Molisch, 2005] Molisch, A. (2005). Ultrawideband propagation channels-theory, measurement, and modeling. Vehicular Technology, IEEE Transactions on, 54(5):1528 - 1545.

[Molisch, 2009] Molisch, A. (2009). Ultra-wide-band propagation channels. Proceedings of the IEEE, 97(2):353-371.

[Molisch, 2011] Molisch, A. (2011). Wireless Communications. Wiley-IEEE Press.

[Molisch et al., 2006] Molisch, A., Cassioli, D., Chong, C.-C., Emami, S., Fort, A., Kannan, B., Karedal, J., Kunisch, J., Schantz, H., Siwiak, K., and Win, M. (2006). A comprehensive standardized model for ultrawideband propagation channels. Antennas and Propagation, IEEE Transactions on, 54(11):3151 -3166.

[Muqaibel et al., 2006] Muqaibel, A., Safaai-Jazi, A., Attiya, A., Woerner, B., and Riad, S. (2006). Pathloss and time dispersion parameters for indoor uwb propagation. Wireless Communications, IEEE Transactions on, 5(3):550 - 559.

[Naqvi and El Zein, 2008] Naqvi, I. and El Zein, G. (2008). Time domain measurements for a time reversal simo system in reverberation chamber and in an indoor environment. In Ultra-Wideband, 2008. ICUWB 2008. IEEE International Conference on, volume 2, pages 211 -214. 
[Naqvi et al., 2010] Naqvi, I. H., Khaleghi, A., and El Zein, G. (2010). Time reversal uwb communication system: a novel modulation scheme with experimental validation. EURASIP J. Wirel. Commun. Netw., 2010:33:1-33:13.

[Nguyen et al., 2006] Nguyen, H. T., Kovacs, I., and Eggers, P. (2006). A time reversal transmission approach for multiuser uwb communications. Antennas and Propagation, IEEE Transactions on, 54(11):3216 -3224.

[Oestges et al., 2004] Oestges, C., Erceg, V., and Paulraj, A. (2004). Propagation modeling of mimo multipolarized fixed wireless channels. Vehicular Technology, IEEE Transactions on, 53(3):644 - 654.

[Pajusco and Pagani, 2009] Pajusco, P. and Pagani, P. (2009). On the use of uniform circular arrays for characterizing uwb time reversal. Antennas and Propagation, IEEE Transactions on, 57(1):102 -109.

[Pallares et al., 2001] Pallares, F., Juan, F., and Juan-Llacer, L. (2001). Analysis of path loss and delay spread at $900 \mathrm{mhz}$ and $2.1 \mathrm{ghz}$ while entering tunnels. Vehicular Technology, IEEE Transactions on, 50(3):767 -776.

[Parsons, 2000] Parsons, J. D. (2000). The Mobile Radio Propagation Channel. J. W. . Sons.

[Peigang et al., 2004] Peigang, J., Shaobo, W., and Huajia, L. (2004). An effective solution of wireless channel sounder and its channel modeling application. In Vehicular Technology Conference, 2004. VTC 2004-Spring. 2004 IEEE 59th, volume 1, pages 249 - 253 Vol.1.

[Qiu et al., 2005] Qiu, R., Liu, H., and Shen, X. (2005). Ultra-wideband for multiple access communications. Communications Magazine, IEEE, 43(2):80 -87.

[Qiu et al., 2006] Qiu, R., Zhou, C., Guo, N., and Zhang, J. (2006). Time reversal with miso for ultrawideband communications: Experimental results. Antennas and Wireless Propagation Letters, IEEE, 5(1):269 -273.

[Quitin et al., 2009a] Quitin, F., Oestges, C., Horlin, F., and De Doncker, P. (2009a). Clustered channel characterization for indoor polarized mimo systems. In Personal, Indoor and Mobile Radio Communications, 2009 IEEE 20th International Symposium on, pages 1064 -1068.

[Quitin et al., 2009b] Quitin, F., Oestges, C., Horlin, F., and De Doncker, P. (2009b). Multipolarized mimo channel characteristics: Analytical study and experimental results. Antennas and Propagation, IEEE Transactions on, 57(9):2739 -2745.

[Quitin et al., 2010] Quitin, F., Oestges, C., Horlin, F., and De Doncker, P. (2010). Polarization measurements and modeling in indoor nlos environments. Wireless Communications, IEEE Transactions on, 9(1):21 -25.

[Rappaport, 1996] Rappaport, T. S. (1996). Wireless Communications, 2nd Ed. Prentice Hall. 
[Richter, 2005] Richter, A. (2005). Estimation of radio channel parameters: Models and algorithms. PhD thesis, Technischen Universität Ilmenau, Germany.

[Richter and Thoma, 2005] Richter, A. and Thoma, R. (2005). Joint maximum likelihood estimation of specular paths and distributed diffuse scattering. In Vehicular Technology Conference, 2005. VTC 2005-Spring. 2005 IEEE 61st, volume 1, pages 11 - 15 Vol. 1.

[Robotics, 2011] Robotics, A. (2011). Xy linear positioners and step motor systems. http://www.arrickrobotics.com/.

[Saghir et al., 2009a] Saghir, H., Heddebaut, M., Elbahhar, F., Rivenq, A., and Rouvaen, J. (2009a). Time-reversal uwb wireless communication-based train control in tunnel. Journal of Communications, 4(4).

[Saghir et al., 2009b] Saghir, H., Heddebaut, M., Elbahhar, F., Rouvaen, J.-M., Menhaj-Rivenq, A., and Ghys, J.-P. (2009b). Train-to-wayside wireless communication in tunnel using ultra-wide-band and time reversal. Transportation Research Part C: Emerging Technologies, 17(1):81 - 97.

[Sakaguchi et al., 2002] Sakaguchi, K., Takada, J., and Araki, K. (2002). A novel architecture for mimo spatio-temporal channel sounder. IEICE Trans. Electron, E85-C:436-441.

[Salous et al., 2005] Salous, S., Filippidis, P., Lewenz, R., Hawkins, I., Razavi-Ghods, N., and Abdallah, M. (2005). Parallel receiver channel sounder for spatial and mimo characterisation of the mobile radio channel. Communications, IEE Proceedings-, 152(6):912 - 918.

[Salous and Razavi-Ghods, 2004] Salous, S. and Razavi-Ghods, N. (June, 2004). Semi-sequential mimo channel measurements in indoor environments. In COST 273 TD(04) 079. Gothenburg, Sweden.

[Schmidt, 1986] Schmidt, R. (1986). Multiple emitter location and signal parameter estimation. Antennas and Propagation, IEEE Transactions on, 34(3):276 - 280.

[Shafi et al., 2006] Shafi, M., Zhang, M., Moustakas, A., Smith, P., Molisch, A., Tufvesson, F., and Simon, S. (2006). Polarized mimo channels in 3-d: models, measurements and mutual information. Selected Areas in Communications, IEEE Journal on, 24(3):514 - 527.

[Shannon, 1948] Shannon, C. E. (1948). A mathematical theory of communication. Bell System Technical Journal, 27:379-423 and 623-656.

[Sibille, 2005] Sibille, A. (2005). Time-domain diversity in ultra-wideband mimo communications. EURASIP J. Appl. Signal Process., 2005:316-327.

[Sibille et al., 2011] Sibille, A., Oestges, C., and Zanella, A. (2011). MIMO: from theory to implementation. Elsevier Inc. 
[Solano García, 2011] Solano García, J. (2011). Improvement of the synchronization of a time-domain wideband mimo measurement system. Technical report, ETSIT - Final Degree Project.

[Stanczak et al., 2001] Stanczak, S., Boche, H., and Haardt, M. (2001). Are las-codes a miracle ? In Global Telecommunications Conference, 2001. GLOBECOM '01. IEEE, volume 1, pages 589-593 vol.1.

[Steinbauer et al., 2001] Steinbauer, M., Molisch, A., and Bonek, E. (2001). The double-directional radio channel. Antennas and Propagation Magazine, IEEE, 43(4):51 -63.

[Takada et al., 2001] Takada, J., Sakaguchi, K., and Araki, K. (2001). Development of high resolution mimo channel sounder for the advanced modeling of wireless channels. In Microwave Conference, 2001. APMC 2001. 2001 Asia-Pacific, volume 2, pages 563 -568 vol.2.

[Telatar, 1995] Telatar, I. E. (1995). Capacity of multi-antenna gaussian channel. Eur. Trans. Telecommun., 10:585-595.

[Thoma et al., 2000] Thoma, R., Hampicke, D., Richter, A., Sommerkorn, G., Schneider, A., Trautwein, U., and Wirnitzer, W. (2000). Identification of time-variant directional mobile radio channels. Instrumentation and Measurement, IEEE Transactions on, 49(2):357 -364.

[Vera Garre, 2011] Vera Garre, C. (2011). Análisis del efecto de la polarización en sistemas mimo-uwb en interiores. Technical report, ETSIT - Final Master Project.

[Wait, 1962] Wait, J. R. (1962). Electromagnetic waves in stratified media. Pergamon Press.

[Wallace et al., 2003] Wallace, J., Jensen, M., Swindlehurst, A., and Jeffs, B. (2003). Experimental characterization of the mimo wireless channel: data acquisition and analysis. Wireless Communications, IEEE Transactions on, 2(2):335 - 343.

[Wang et al., 2005] Wang, L.-C., Liu, W.-C., and Shieh, K.-J. (2005). On the performance of using multiple transmit and receive antennas in pulse-based ultrawideband systems. Wireless Communications, IEEE Transactions on, 4(6):2738 - 2750.

[Win et al., 2009] Win, M., Dardari, D., Molisch, A., Wiesbeck, W., and Zhang, J. (2009). History and applications of uwb. Proceedings of the IEEE, 97(2):198 -204.

[Win and Scholtz, 1998] Win, M. and Scholtz, R. (1998). Impulse radio: how it works. Communications Letters, IEEE, 2(2):36 -38.

[Winters, 1987] Winters, J. (1987). On the capacity of radio communication systems with diversity in a rayleigh fading environment. Selected Areas in Communications, IEEE Journal on, 5(5):871 - 878.

[Wirnitzer et al., 2001] Wirnitzer, W., Bruckner, D., Thoma, R., Sommerkorn, G., and Hampicke, D. (2001). Broadband vector channel sounder for mimo channel measurement. In MIMO: Communications Systems from Concept to Implementations (Ref. No. 2001/175), IEE Seminar on, pages $17 / 1-17 / 4$. 
[Wonsop et al., 2007] Wonsop, K., Park, J. J., Kim, M.-D., and Chung, H. K. (2007). Time-division multiplexing based mimo channel sounder using loosely synchronous codes. In Vehicular Technology Conference, 2007. VTC-2007 Fall. 2007 IEEE 66th, pages 897 -901.

[Yang and Giannakis, 2004] Yang, L. and Giannakis, G. (2004). Analog space-time coding for multiantenna ultra-wideband transmissions. Communications, IEEE Transactions on, 52(3):507 517.

[Zeiler, 1999] Zeiler, M. (1999). Modeling Our World. The ESRI Guide to Geodatabase Design. Environmental Systems Research Institute, Inc.

[Zhang and Hwang, 1998] Zhang, Y. and Hwang, Y. (1998). Characterization of uhf radio propagation channels in tunnel environments for microcellular and personal communications. Vehicular Technology, IEEE Transactions on, 47(1):283 -296.

[Zhao et al., 2003] Zhao, X., Geng, S., Vuokko, L., Kivinen, J., and Vainikainen, P. (2003). Polarization behaviours at 2, 5 and $60 \mathrm{ghz}$ for indoor mobile communications. Wireless Personal Communications, 27:99-115.

[Zheng and Kaiser, 2004] Zheng, F. and Kaiser, T. (2004). On the evaluation of channel capacity of multi-antenna uwb indoor wireless systems. In Spread Spectrum Techniques and Applications, 2004 IEEE Eighth International Symposium on, pages 525 - 529.

[Zheng and Kaiser, 2008] Zheng, F. and Kaiser, T. (2008). On the evaluation of channel capacity of uwb indoor wireless systems. Signal Processing, IEEE Transactions on, 56(12):6106 -6113.

[Zhou et al., 2006] Zhou, C., Guo, N., and Qiu, R. (2006). Experimental results on multipleinput single-output (miso) time reversal for uwb systems in an office environment. In Military Communications Conference, 2006. MILCOM 2006. IEEE, pages 1 -6. 


\section{GLOSSARY}

$3 G$

Third Generation.

$4 G$

Fourth Generation.

AoA

Angle of Arrival.

AoD

Angle of Departure.

APS

Azimuth Power Spectrum.

\section{BAN}

Body Area Networks.

\section{CAG}

Automatic Gain Control.

\section{CCDF}

Complementary Cumulative Distribution Function. 


\section{CDF}

Cumulative Distribution Function.

\section{CDM}

Code-Division Multiplexing.

\section{CEPT}

European Conference of Postal and Telecommunication Administrations.

\section{CIR}

Channel Impulse Response.

\section{CW}

Continuous Wave.

\section{DoA}

Direction of Arrival.

\section{DoD}

Direction of Departure.

\section{EIRP}

Equivalent Isotropic Radiated Power.

\section{FCC}

Federal Communications Commission.

\section{FDM}

Frequency-Division Multiplexing.

\section{GIS}

Geographic Information System.

\section{GPIB}

General Purpose Interface Bus.

\section{GPS}

Global Positioning System.

\section{IDFT}

Inverse Discrete Fourier Transform.

IF

Intermediate Frequency. 


\section{IFFT}

Inverse Fast Fourier Transform.

IFW

Interference Free Window.

IR

Impulse Radio.

LA

Large Area.

\section{LAN}

Local Area Network.

\section{LAS}

Large Area Synchronized.

\section{LoS}

Line-of-Sight.

LS

Loosely Synchronous.

\section{MIMO}

Multiple-Input Multiple-Output.

MISO

Multiple-Input Single-Output.

MPC

Multipath Contribution.

\section{MVNA}

Multiport Vector Network Analyzer.

\section{NLoS}

Non Line-of-Sight.

\section{OFDM}

Orthogonal Frequency Division Mutiplexing.

\section{PAN}

Personal Area Networks. 


\section{PDP}

Power-Delay Profile.

PN

Pseudo Noise.

\section{PRBS}

Pseudo-Random Binary Signals.

\section{QS-CDMA}

Quasi-Synchronous Code Division Multiple Access.

\section{RMS}

Root Mean Square.

$\mathrm{RX}$

Receiver.

\section{SIMO}

Single-Input Multiple-Output.

SISO

Single-Input Single-Output.

\section{SNR}

Signal-to-Noise Ratio.

\section{TDM}

Time-Division Multiplexing.

TIN

Triangulated Irregular Network.

TR

Time Reversal.

TX

Transmitter.

UWB

Ultra Wide-Band.

VNA

Vector Network Analyzer. 
WLAN

Wireless Local Area Network.

WSN

Wireless Sensor Networks.

XPD

Cross Polar Discrimination Factor. 